\title{
Development of Aptasensors for Steroidal Hormones
}

\author{
by
}

Omar Ahmed Alsager

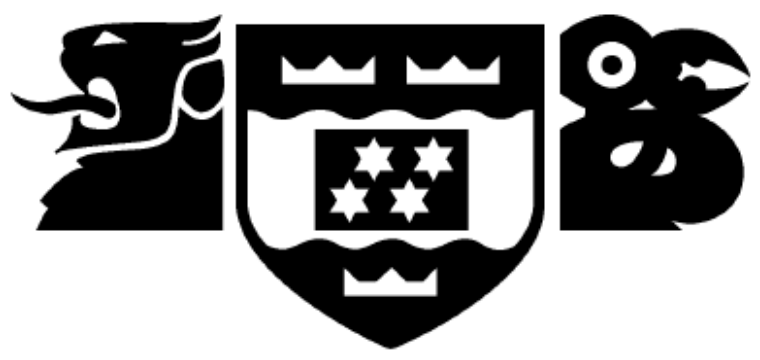

Victoria University of Wellington 


\begin{abstract}
Aptamers are synthetic nucleic acid single stranded (ss)DNAs or RNAs that can bind with high affinity and specificity to a broad range of targets, including proteins and low molecular weight molecules. This work presents the design, development and implementation of novel aptamer based sensors (aptasensors) for the detection of a target of environmental and medical significance - 17- $\beta$ estradiol (E2). By combining a previously isolated E2 binding 75-mer ssDNA aptamer with a variety of different signal transducers, E2 was successfully detected and quantified below the environmental and biological relevant concentrations. By applying the same aptamer to different sensor formats, the advantages and disadvantages of each signal transduction mechanism were compared.
\end{abstract}

Target-induced conformational switch within an aptamer molecule can be transduced via labelling different sections of the aptamer with pairs of fluorescent dyes or with a redox probe, however those strategies require detailed knowledge of specific aptamer conformations and target interaction sites. Herein, a label free method is developed - size based aptasensor described in Chapter 2. The new method only depends on the general property that small molecule binding aptamers adopt a more compact folded structure when they bind to their target. Dynamic light scattering (DLS) and tunable resistive pulse sensing (TRPS) were used to probe recognition events between E2 and aptamers conjugated to carboxylated polystyrene nanoparticles (NPs). Upon E2 recognition, a distinct reduction in size and a less negative surface potential of the conjugated particles were observed, which can be correlated to the concentration of E2 in the lower nanomolar range (as low as $5 \mathrm{nM}$ ).

On-site monitoring of E2 requires rapid and sensitive screening methods with minimal instrumentation. Previously, gold nanoparticles (AuNPs) were exploited in the construction of colorimetric aptasensors for different targets. Aggregation assays produce colorimetric signals observed by naked-eye when target-bound aptamers dissociate from AuNP surfaces, triggering aggregation. However, it is unknown how the length of aptamer sequences affects their dissociation from AuNP surfaces and subsequent aggregation. Chapter 3 demonstrates the benefit 
of editing aptamer sequences with specific regard to the way signals are transduced in AuNP based colorimetric assays. The 20 flanking nucleotides to the 35-mer inner core of the parent 75-mer aptamer were eliminated. The 35-mer aptamer has a lower dissociation constant $K_{\mathrm{D}}(14 \mathrm{nM}$ vs. $25 \mathrm{nM})$, improved discrimination against other steroidal molecules and greatly improve the sensitivity for E2 detection from $5 \mathrm{nM}$ to $200 \mathrm{pM}$. In fact, this simple strategy enabled facile detection of $\mathrm{E} 2$ in urine at $5 \mathrm{nM}$, approaching levels of biological relevance.

There is a pressing demand for methods with accurate and rapid performance to detect and quantify E2, at levels comparable or even below the biological concentrations to eliminate pre-concentration and sample purification process. Existing electrochemical aptasensors feature DNA probes covalently tethered to various surfaces including gold and conducting polymer electrode. An electrochemical impedance spectroscopy (EIS) based sensor was created using nanoporous conducting polymer electrodes functionalized with the 75-mer aptamer. The one $\mathrm{fM}$ detection limit found is one order of magnitude lower than the recorded biological level. As a novel alternative approach, sensing electrodes were also created via the non-specific adsorption of the 35-mer onto Au and Au nanoparticle electrodes. This approach, described in Chapter 4, led to the same level of detection as the conducting polymer aptasensor, but via a mechanism with similarities to the colorimetric sensor. Non-specific adsorption of aptamers to Au was found to play additional favourable roles including self-passivation and stabilization of $\mathrm{Au}$ nanoparticle based electrodes. Sensing with this format might remove the need for laborious surface passivation with alkylthiol molecules encountered with the conventional covalent attachment of the DNAs through thiol-linkers.

In general, the reported aptasensors provide efficient means to detect the steroidal molecule E2 as well as advance the understanding of aptasensors by comparing the performance of the same aptamer in various sensing platforms. Long aptamers sequences appeared to be more efficient in signal transduction when specific surface tethering is involved, as in the size-based assay, and the electrochemical assay with aptamers covalently tethered to the electrode. Here, the non-binding flanking nucleotides, i.e. nucleotides adjacent to the target binding pocket, appeared to amplify the sensing signals. However, shorter truncated sequences showed better performance when signal generation depends on surface dissociation of non-specifically adsorbed aptamer sequences, as in the colorimetric assay, and the electrochemical sensor constructed from adsorbed aptamers. These insights can be readily applied to aptasensors for the growing range of targets. 


\section{Acknowledgment}

Special gratitude and thanks are extended to my advisor Dr. Justin Hodgkiss. He has been a tremendous mentor for me. I would like to thank him for encouraging my research and for helping me to develop my scientific skills. His advice and guidance have been priceless. I would also like to thank Dr. Geoff Willmott for assisting in the development of the size based aptasensor and for useful discussions which helped me in many ways.

Special thanks to my collaborators, Dr. Shalen Kumar, Bicheng Zhu, Professor Jadranka Travas-Sejdic, and Professor Ken McNatty. Words cannot express how grateful I am to them. They enriched my knowledge and made my experience exciting. Professor Jadranka, thank you for allowing me to spend time with the electrochemical instruments and enabling me to attain better understanding of my aptasensors. Many tanks go to Dr. Christine Stockum for providing biological samples. My deepest gratitude also goes to all the staff members of IZON Science Ltd., especially Hans Van der Voorn and Dr. Darby Kozak for the training and useful discussions regarding the size based aptasensor.

Furthermore, I wish to express sincere appreciation to my mother and father; they offered support, inspiration, and encouragement that no body else could have. I thank my beloved wife Rania for being by my side. Without your support, it would have been very difficult to accomplish what I have achieved. Appreciation is also expressed to my family for their motivational talks. I greatly thank my friends here in New Zealand and in Saudi Arabia for their valued assistance during the entire period of my $\mathrm{PhD}$, particularly those who helped me in using LATEX to write my thesis; Joe Gallaher, Ahmed Alshek, and Jawad Mirza.

I would like to acknowledge the support from my country, particularly the King Abdul Aziz City for Science and Technology for giving me this opportunity by providing the scholarship which enabled this thesis. 


\section{Research outputs}

\section{Patent applications}

- "Novel bio-sensor for the detection of small molecules" Hodgkiss, J. M.; McNatty, K. P.; Alsager, O. A.; Kumar, S. NZ Patent application number 612582, 2013.

- "Polynucelotide sequence" Hodgkiss, J. M.; McNatty, K. P.; Alsager, O. A.; Kumar, S. NZ Patent application number 624985, 2014.

- "Quantification methods in Samples" Travas-Sejdic, J.; Hodgkiss, J. M.; Zhu, B.; Alsager, O. A. NZ Patent application 701521, 2014.

\section{Journal articles}

- "Small molecule detection in solution via the size contraction response of aptamer functionalized nanoparticles" Alsager, O. A.; Kumar, S.; Willmott, G. R.; McNatty, K. P.; and Hodgkiss, J. M. (Biosensors and Bioelectronics 2014, 57, 262-268.)

- "Ultrasensitive colorimetric detection of 17- $\beta$-estradiol: The effect of shortening DNA aptamer sequences" Alsager, O. A.; Kumar, S.; Zhu, B.; TravasSejdic, J.; McNatty, K. P.; and Hodgkiss, J. M. (Analytical Chemistry, 2015, 87, 4201-4209).

- "Label-free electrochemical aptasensor for femtomolar detection of 17- $\beta$ estradiol" Zhu, B.; Alsager, O. A.; Kumar, S.; Hodgkiss, J. M.; and TravasSejdic, J. (Biosensors and Bioelectronics,2015, 70, 398-403).

- "Non-specific adsorption versus covalent coupling of DNA aptamer probes on gold electrodes for sensing applications" Alsager, O. A.; Zhu, B.; TravasSejdic, J.; and Hodgkiss, J. M. (Manuscript prepared for submission once patent has been filed). 
- "Base dependent adsorption of single-stranded homo-oligonucleotides to gold nanoparticles" Alsager, O. A.; Andreassend, S. K.; Zhu, B.; TravasSejdic, J.; and Hodgkiss, J. M. (Submitted).

\section{Conference posters}

- "Colorimetric Hormone Sensing with Gold Nanoparticles and DNA Aptamers", Gordon Research conference (GRC), Noble Metal Nanoparticles at Mount Holyoke College in South Hadley, MA, USA (14-20/06/2014).

- "Development of Aptasensors for Steroidal Hormones", Gordon Research conference (GRC), Bioanalytical Sensors at Salve Regina University in Newport, RI, USA (21-27/06/2014).

\section{Conference presentations}

- "Probing the behavior of nanoparticles conjugated with oligonucleotides", AMN6 international conference in Auckland, New Zealand (11-16/02/2013).

- "Small molecule detection via the size contraction response of aptamer functionalized nanoparticles", Izon international symposium in Boston, USA (21-23/10/2013). 


\section{Abbreviations and common terms}

Aptamers Nucleic acid species with specific binding functions

AuNPs Gold nanoparticles

BPA Bisphenol A

BPF 4, 4'-methylenediphenol

BWB Binding and washing buffer

CC Chronocoulometry

CD Circular dichroism

CV Cyclic voltammetry

DLS Dynamic light scattering

dsDNA Double-stranded DNA

DNA Deoxyribonucleic acid

ECP Electrochemically conducting polymer

EDCs Endocrine Disrupting Compounds

EDC/NHS N-(3-dimethylaminopropyl) N-ethylcarbodiimide/N hydroxy-succinimide

EIS Electrochemical impedance spectroscopy

E2 $17-\beta$ estradiol

FWHM Full width at half maximum

GCE Glassy carbon electrode

$\mathbf{K}_{D}$ Dissociation constant

LOD Limit of detection

MES 2-(N-morpholino) ethanesulfonic acid (MES) 
NPs Nanoparticles

PBS Phosphate buffered saline

P4 Progesterone

T Testosterone

TEM Transmission Electron Microscopy

TRPS Tunable resistive pulse sensing

PALS Phase analysis light scattering

$\mathbf{R}_{C T}$ Charge transfer resistance

SELEX Systematic evolution of ligands by exponential enrichment

SEM Scanning Electron Microscopy

ssDNA Single-stranded DNA

QD Quantum dot 


\section{Contents}

Acknowledgment i

Research outputs ii

Abbreviations and common terms iv

\begin{tabular}{lll}
\hline & Introduction & 1
\end{tabular}

1.1 Background $\ldots \ldots \ldots \ldots \ldots \ldots$

1.1 .1 Biosensors . . . . . . . . . . . . . . . 1

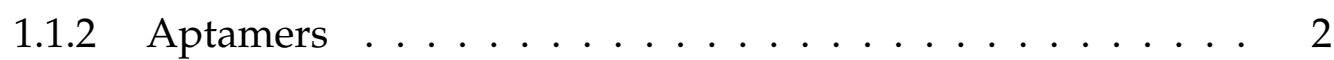

1.1 .3 Aptamers in biosensors . . . . . . . . . . . . . . . . . 6

1.2 Research problems and objectives . . . . . . . . . . . . . . 20

$1.2 .1 \quad$ Estrogenic derivatives . . . . . . . . . . . . . 20

1.2 .2 Research objectives and thesis overview . . . . . . . . . . 22

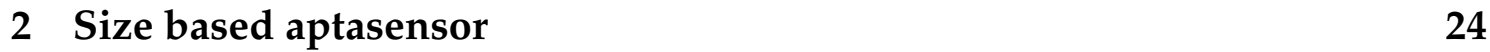

2.1 Abstract . . . . . . . . . . . . . . . . . . . . 24

2.2 Introduction . . . . . . . . . . . . . . . . . . 24

2.3 Overview of TRPS and light scattering techniques . . . . . . . . 26

2.4 Results and discussion . . . . . . . . . . . . . . . . . . 31

2.4 .1 Aptamer selection and characterisation . . . . . . . . . 31

2.4 .2 Generation and characterization of NP-aptamer . . . . . . . 35

2.4 .3 Detection of E2 . . . . . . . . . . . . . . . . 40

2.4 .4 Generality of the sensor . . . . . . . . . . . . 43

2.5 Chapter conclusion . . . . . . . . . . . . . . . . 44

2.6 Experimental section . . . . . . . . . . . . . . . 45

3 Colorimetric aptasensor $\quad 50$

3.1 Abstract .............................. 50

3.2 Introduction $\ldots \ldots \ldots \ldots \ldots \ldots \ldots \ldots$

3.3 Results and discussion . . . . . . . . . . . . . . . . . 53

3.4 Chapter conclusion . . . . . . . . . . . . . . 66

3.5 Experimental section . . . . . . . . . . . . . . 67 
\begin{tabular}{|lll}
4 & Electrochemical impedance spectroscopy based aptasensors & 72
\end{tabular}

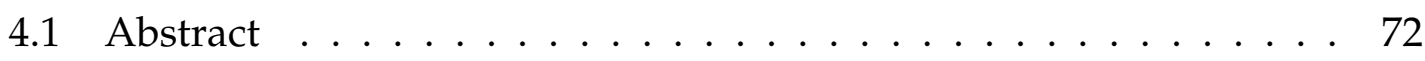

4.2 Overview of EIS . . . . . . . . . . . . . . . . . . . . . 73

$4.3 \quad$ E2 aptasensing based on conducting polymer . . . . . . . . . . . 75

4.3 .1 Introduction . . . . . . . . . . . . . 75

4.3 .2 Results and discussion . . . . . . . . . . . . . 76

$4.4 \quad$ E2 aptasensing based on the non-specifically adsorbed aptamer . . 80

4.4 .1 Introduction . . . . . . . . . . . . . . . 80

4.4 .2 Results and discussion . . . . . . . . . . . . . 82

4.5 Chapter conclusion . . . . . . . . . . . . . . . . 97

4.6 Experimental section $\ldots \ldots \ldots \ldots$. . . . . . . . . . . . 98

5 Evaluation and comparisons of the developed aptasensors $\quad 102$

5.0 .1 Comparison between the developed aptasensors. . . . . . . 102

5.0 .2 The role of excess flanking nucleotides in enhancing or suppressing the sensing signals . . . . . . . . . . . . . . 104

5.0 .3 Aptasensors developed in this thesis versus literature meth-

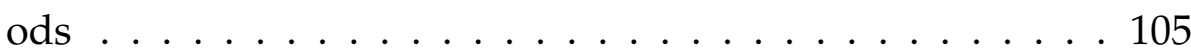

6 Conclusions and Future work 107

6.1 Conclusions . . . . . . . . . . . . . . . . 107

6.2 Future work . . . . . . . . . . . . . . . . . . . . 109

\begin{tabular}{|ll|}
\hline A Supplementary information of size based sensor & 111
\end{tabular}

\begin{tabular}{ll}
\hline B Colorimetric aptasensor & $\mathbf{1 1 2}$
\end{tabular}

B.1 Detection with the 75-mer and Truncated Aptamers . . . . . . . . . 112

B.1.1 Spectra for aptamer responses to E2 concentrations . . . . . 112

B.1.2 Specificity examination . . . . . . . . . . . 113

B.2 CD characterisation of E2 recognition. . . . . . . . . . . . . . . 114

B.3 Urine samples: Determination of ionic strength and detection of E2 116

B.4 $K_{\mathrm{D}}$ measurements . . . . . . . . . . . . . . . . . 118

\begin{tabular}{ll}
\hline C Supplementary information of EIS based sensor & 119
\end{tabular}

C.1 Surface area of AuNPs on glassy carbon electrode . . . . . . . . . 119

C.2 Figures for EIS AuNP electrode . . . . . . . . . . . . . . . . . 120

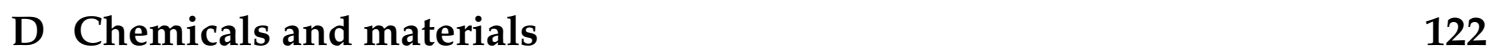




\section{List of Tables}

$2.1 \zeta$-potential characterisation of the size based sensor $\ldots . . \ldots 36$

$3.1 \zeta$-potential values for the 75-mer and truncated aptamers with AuNPs 60

3.2 Surface densities for the 75-mer and truncated aptamers on AuNP electrodes ........................ 64

5.1 Comparisons of various methods for the detection of E2 including aptasensors developed in this thesis. . . . . . . . . . . . . 106

D.1 Sequences used in this thesis . . . . . . . . . . . . . . . 124 


\section{List of Figures}

1.1 Schematic of the classification of biosensors schemes. . . . . . . . 2

1.2 Structure of DNA . . . . . . . . . . . . . . . . 3

1.3 Typical round of SELEX $\ldots \ldots \ldots \ldots \ldots \ldots$

1.4 Schematic representation of aptamers functionality $\ldots \ldots \ldots .5$

1.5 Strategies employed in fluorescence based aptasensing . . . . . . 8

1.6 Surface plasmon resonance of AuNPs $\ldots \ldots \ldots \ldots \ldots$

1.7 Strategies employed in colorimetric based aptasensing . . . . . . . 11

1.8 Strategies employed in lateral flow aptasensing. . . . . . . . . . . 13

1.9 Strategies employed in electrochemical aptasensing . . . . . . . . 15

1.10 Strategies employed in size based aptasensing . . . . . . . . . . . 18

2.1 Overview of qNano system $\ldots \ldots \ldots \ldots \ldots \ldots \ldots$

2.2 Electrical double layer and Zeta potential . . . . . . . . . 30

2.3 Secondary structure of the 75-mer aptamer $\ldots \ldots \ldots \ldots$

$2.4 \quad K_{\mathrm{D}}$ of the 75 -mer aptamer $\ldots \ldots \ldots \ldots \ldots \ldots$

2.5 Characterisation of the 75-mer aptamer $\ldots \ldots \ldots \ldots \ldots$

2.6 Surface coverage of the 75-mer on carboxylated polystyrene NPs . 38

2.7 SEM characterisation of 75-mer aptamer coupling to NPS . . . . . 39

2.8 Size based characterisation of aptamer coupling and E2 sensing . . 40

2.9 Linearity and specificity of the size based aptasensor . . . . . . . 42

2.10 Generality of the size based aptasensor $\ldots \ldots \ldots \ldots$. . . . 44

3.1 Sensitivity optimisation for sensing E2 with the 75-mer aptamer . . 54

3.2 TEM and DLS characterisation of E2 sensing . . . . . . . . 55

3.3 Colorimetric sensing of E2 with the 75-mer aptamer . . . . . . . 56

$3.4 \quad$ M-fold secondary structure and CD spectra of the 75-mer aptamer and truncated sequences $\ldots \ldots \ldots \ldots \ldots \ldots$

3.5 Determination of $K_{\mathrm{D}}$ for the 22-mer and 35-mer aptamers. . . . . . 59

3.6 Colorimetric sensing of E2 with the 35-mer aptamer . . . . . . . 61

3.7 Colorimetric sensing of E2 with the 22-mer aptamer . . . . . . . . 62

3.8 Chronocoulometry surface density for the 75-mer, 35-mer, and 22mer

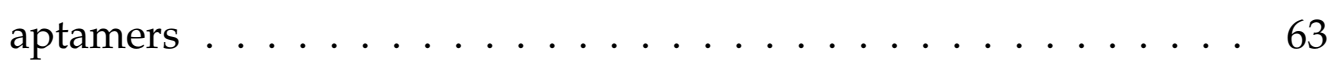


3.9 Sensing E2 in spiked urine samples with the 75-mer and 35-mer

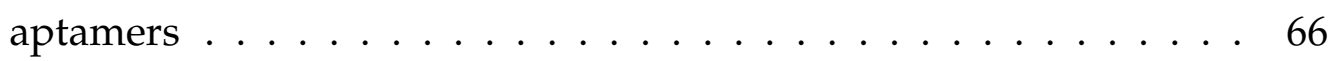

4.1 Principles of electrochemical impedance spectroscopy . . . . . . . . 74

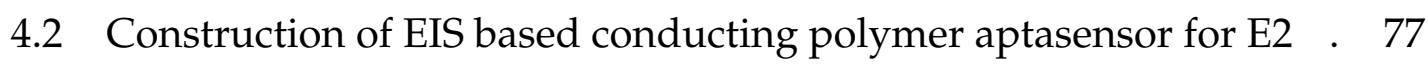

4.3 Sensing E2 with EIS based conducting polymer aptasensor . . . . . 78

4.4 Comparison of sensing E2 with EIS based conducting polymer us-

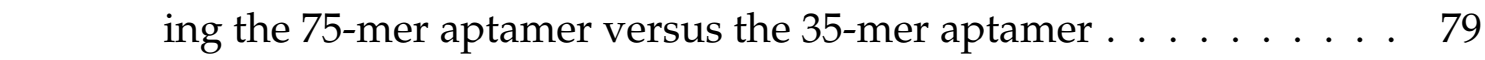

4.5 EIS of covalent coupling and non-specific adsorption of aptamers to flat Au electrode . . . . . . . . . . . . . . . 82

4.6 SEM, EIS, and CV Characterisation of AuNP electrode. . . . . . . . 85

$4.7 \quad$ EIS and CV of covalent coupling and non-specific adsorption of

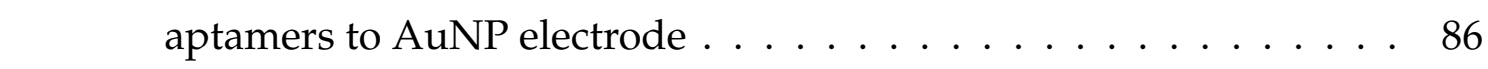

$\begin{array}{lll}4.8 & \text { Generality of EIS decreased signals with different DNA sequences. } & 87\end{array}$

4.9 Chronocoulometry surface densities of covalent coupling and non-

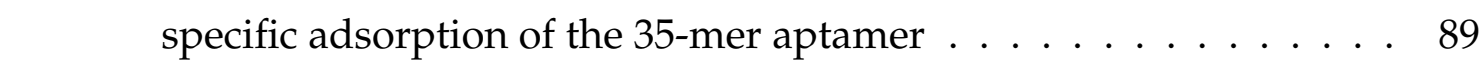

4.10 Different surface treatments of AuNP electrode with the covalently

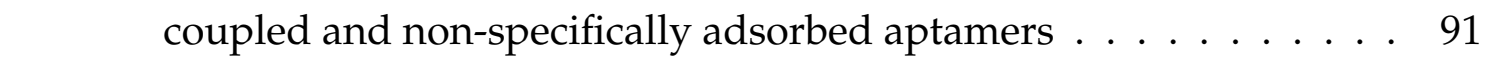

4.11 Sensing E2 on flat Au electrode with the covalently coupled and non-specifically adsorbed 35-mer aptamer . . . . . . . . . . . . . 92

4.12 Sensing E2 on AuNP electrodes with the covalently coupled and non-specifically adsorbed 35-mer aptamer . . . . . . . . . . . . 94

4.13 Chronocoulometry characterisation of E2 detection with the adsorbed 75-mer and 35-mer aptamers . . . . . . . . . . . . . 95

4.14 E2 detection in spiked rate urine samples using non-specifically

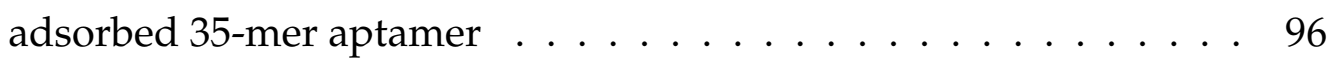

4.15 Comparison of EIS based E2 detection using non-specifically ad-

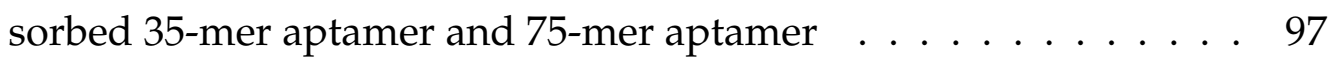

A.1 Effect of IGEPAL on the TRPS count rate . . . . . . . . . . . . . . . . 111

B.1 UV-vis spectra for E2 sensing with the 35-mer and 22-mer aptamers 112

B.2 UV-vis spectra for specificity examination of the 75-mer aptamer a and truncated versions . . . . . . . . . . . . . . . . . . 113

B.3 CD characterisation of E2 sensing with the 75-mer aptamer and truncated versions . . . . . . . . . . . . . . 114

B.4 M-fold secondary structure and CD of poly T20-(35-mer)-poly T20

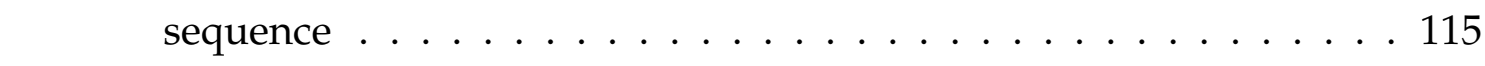

B.5 Conductivity measurement to estimate the ionic strength of rat urine samples . . . . . . . . . . . . . . . . . 116

B.6 $\quad$ Sensing E2 in rat urine samples with the 35-mer aptamer . . . . . 116 
B.7 Specificity examination of the 35-mer aptamer in spiked rate urine samples . . . . . . . . . . . . . . . . . . . . 117

B.8 $\quad$ Sensing E2 in spiked rat urine samples with the 75-mer aptamer . . 117

B.9 Supplementary information for $K_{\mathrm{D}}$ measurements for the 35-mer and 22-mer aptamers . . . . . . . . . . . . . . . . . . . . . 118

C.1 Stability of AuNP electrode and modified AuNP electrodes against washing cycles of buffer $\ldots \ldots$. . . . . . . . . . . . 120

C.2 Nyquist plots for sensing E2 on flat and AuNP electrodes . . . . . . 121

C.3 Control experiment of exposing the 35-mer aptamer to GCE . . . . 121 


\section{List of Schemes}

1.1 Chemical structures of the abundant estrogenic EDCs in the environment. . . . . . . . . . . . . . . . . . . . . 21

2.1 Schematic overview of E2 size based aptasensor . . . . . . . . 26

2.2 EDC /NHS coupling chemistry . . . . . . . . . . . . 36

3.1 Colorimetric sensing scheme $\ldots \ldots \ldots \ldots$. . . . . . . . 52

4.1 Covalent coupling and non-specific adsorption of aptamers to $\mathrm{Au}$ surfaces . . . . . . . . . . . . . . . . . 81

4.2 Change in ion distribution with covalent coupling and non-specific adsorption of aptamers $\ldots \ldots \ldots \ldots \ldots \ldots 4$ 


\section{Chapter 1}

\section{Introduction}

\subsection{Background}

This chapter will firstly introduce the concept of biosensing and the various avenues within this field. The use of biomolecules, specifically aptamers, as receptors in biosensing will be reviewed as well as the implementation of aptamers with various sensing schemes. Research problems and objectives will be established at the end of this chapter.

\subsubsection{Biosensors}

A biosensor is defined as an analytical device that joins a signal transducer with a biological, or a biologically driven, sensitive and selective recognition element. When a given target analyte interacts with the recognition element, a binding event occurs, generating a signal which is related to the concentration of the target at the transduction level. Biosensors are constructed to measure targets of medical and environmental significance at a cost comparable to, or better than, traditional analytical techniques. ${ }^{1}$ Biosensors can be classified in two categories: based on the type of transduction technique being optical, electrochemical, mass, size and others or based on the type of the recognition element being antibodies, enzymes, aptamers and others as illustrated in Fig. 1.1. 


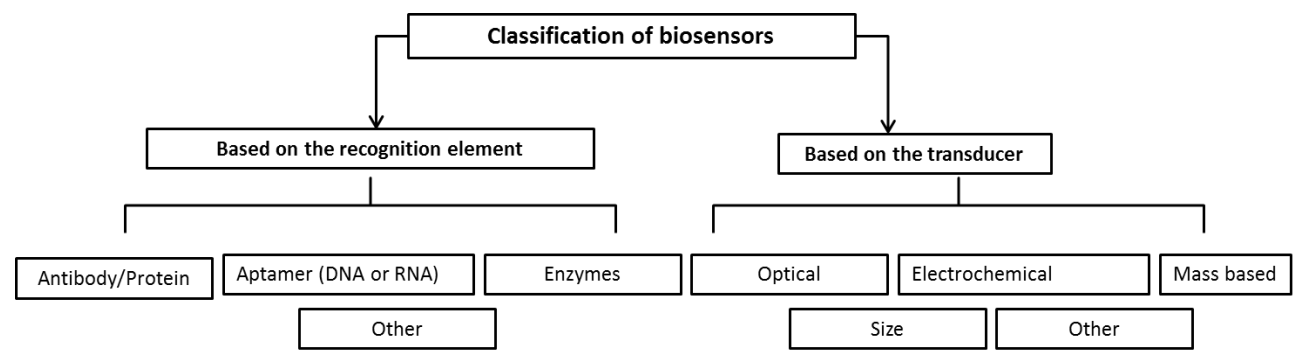

Figure 1.1: Schematic of the classification of biosensors schemes.

Amongst the other recognition elements, aptamers are an emerging class of molecules with several important advantages, reviewed in Section 1.1.2. More importantly, and of relevance to this project, aptamers are efficient in binding not only to large targets, such as proteins ${ }^{2}$ and cells ${ }^{3}$ but also to small molecules, such as nucleotides, organic dyes, amino acids, ${ }^{4}$ metal ions, ${ }^{5}$ and molecules of environmental significance. ${ }^{6-8}$ However, antibodies and other recognition element classes are generally efficient in binding large molecules which are more structurally complicated. ${ }^{9}$ Therefore, aptamers open great opportunities to detect small molecules in a sensitive, selective way, with low detection limits. ${ }^{10}$ Aptamers have been combined with a diverse range of transduction methods (shown in Fig. 1.1) targeting a wide range of analytes of medical, biochemical, food and environmental importance. 4 [6]10, 12

Selected transduction methods that integrate aptamers and their constructions will be reviewed in Section 1.1.3. Nanomaterials exhibit novel optical, electrochemical and surface properties making them well-suited for sensing applications. ${ }^{13}$ In recent years, there has been great progress in the investigation of the potential integration of nanomaterials in biosensors. Attempts were made to improve the sensitivity and eliminate the nonspecific binding between the recognition elements and the sensor surfaces. ${ }^{14}$ Various types of nanomaterials such as AuNPs, carbon nanotubes, organic nanoparticles, magnetic nanoparticles and quantum dots (QD) have been frequently incorporated in biosensors. ${ }^{15}$ Selected examples, of relevance to this thesis, of the integration of nanomaterial with aptamers will be briefly reviewed in Section 1.1.3 of the present Chapter.

\subsubsection{Aptamers}

Before the discussion of aptamer background and various processes involved, the basic properties of deoxyribonucleic acid (DNA) are reviewed. It should be noted that the focus of this thesis is the use of ssDNA aptamers, therefore, RNAs are not 
reviewed.

\section{Overview of DNA}

From a chemical point of view, DNA is a polymer consisting of a four types of deoxyribonucleotide monomers distinguished by their nitrogen bases, adenine $(A)$, thymine $(\mathrm{T})$, cytosine $(\mathrm{C})$, and guanine $(\mathrm{G})$ (each monomer is made of a nitrogen base, a deoxyribose sugar, and one phosphate group). ${ }^{[16}$ In the doublestranded structure (dsDNA), the nitrogen bases are embedded and only the negatively charged phosphate groups are exposed for maximal protection of genetic information. However, single-stranded (ss)DNAs, Fig. 1.2, expose both phosphate groups and nitrogen bases with less structural rigidity than dsDNA. At neutral pHs, the nitrogen bases are non-charged and the DNA is highly negatively charged with number of backbone charges equal to $n-1$ where $n$ is the number of monomers ( $\mathrm{pK}_{\mathrm{a}}$ of phosphate backbone is around 2). ${ }^{16}$ ssDNAs can be chemically synthesised with high throughput, with arbitrary sequences becoming available and able to be amplified (generation of identical copies) in vitro by polymerase chain reaction (PCR). 17

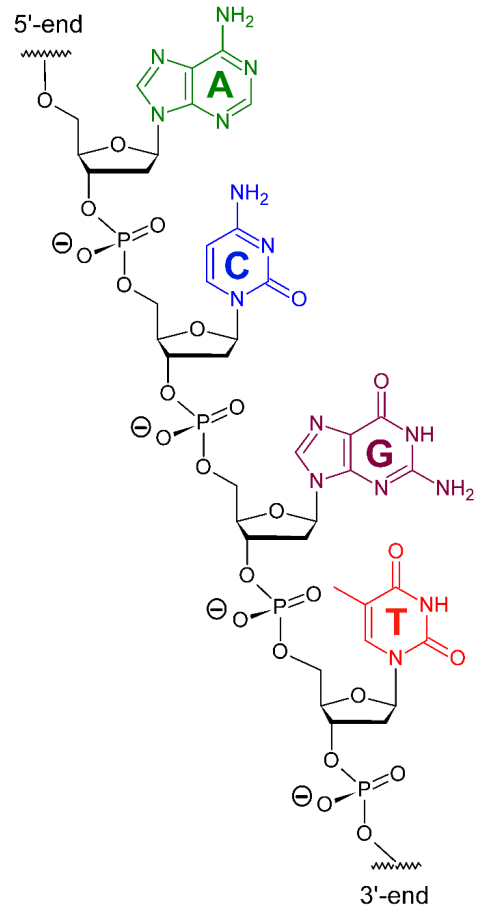

Figure 1.2: ssDNA containing the nitrogen bases. 5' end and $3^{\prime}$ end indicate the beginning and ending points of the ssDNA. 


\section{Aptamer selection and application}

Aptamers are single stranded oligonucleotides (ssDNA or RNA) analogous to antibodies. They were discovered in 1990 by two independent research groups, Tuerk and Gold and Ellington and Szostak. ${ }^{1819}$ Aptamers are generated by a revolutionary process called systematic evolution of ligands by exponential enrichment (SELEX) to specifically and selectively bind targets. SELEX involves 10-20 rounds of the in vitro selection processes, starting with a random pool of synthetic and random oligonucleotide strands $\left(10^{14}-10^{15}\right) .4[8 \mid 1120$

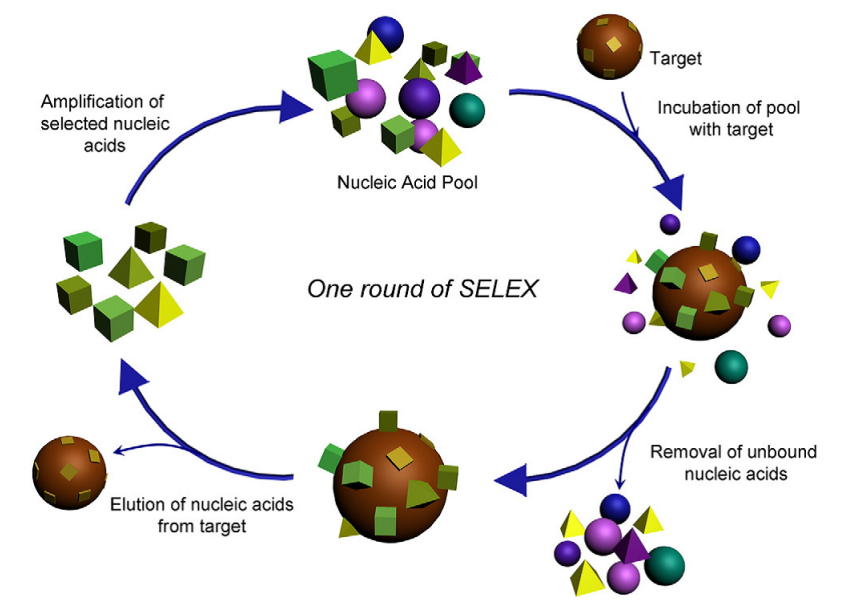

Figure 1.3: Typical round of SELEX starting with $10^{14}-10^{15}$ random DNA sequences and the incubation with the immobilised target molecule. Unbound sequences are removed and the bound ones are eluted and amplified for further SELEX selection rounds. ${ }^{4}$ Figure is reported with permission from Elsevier, reference. ${ }^{4}$

Fig. 1.3 shows a typical round of SELEX, where the target analyte is immobilised on a solid surface followed by incubation with a pool of oligonucleotides. After incubation, sequences that have affinity towards the target will bind and the unbound sequences are washed (removed) from the reaction mixture. The bound sequences are eluted and subjected to PCR amplification. After a given number of SELEX rounds, the ssDNA library is refined and only those few oligonucleotides with the highest affinity are selected and sequenced. Finally, the dissociation constants $\left(K_{\mathrm{D}} \mathrm{s}\right)$ are determined for those sequences interacting with the target. $\underline{4}$

Aptamers (also called functional nucleic acids) can interact and bind to a specific analyte, resulting in a conformation change (conformational transition). Like proteins, aptamers can fold into secondary and in some cases tertiary structures characterized by stems and loops that could perform a variety of roles including target recognition, ${ }^{21}$ Fig. 1.4 (the example given in the figure is for an aptamer interacting with an arbitrary large target). Target recognition could result from structure 
compatibility, stacking of aromatic rings, electrostatic and van der Waals interactions, and hydrogen bondings, or from a combination of these effects. ${ }^{.22}$ Since their first discovery, aptamers have been selected to numerous targets of medical, industrial, and environmental importance ranging in molecular size from ions to entire cells. Of the existing aptamers, $71 \%$ have been selected for proteins, 19\% for small molecules, $7 \%$ for virus and the remaining for ions. ${ }^{9}$

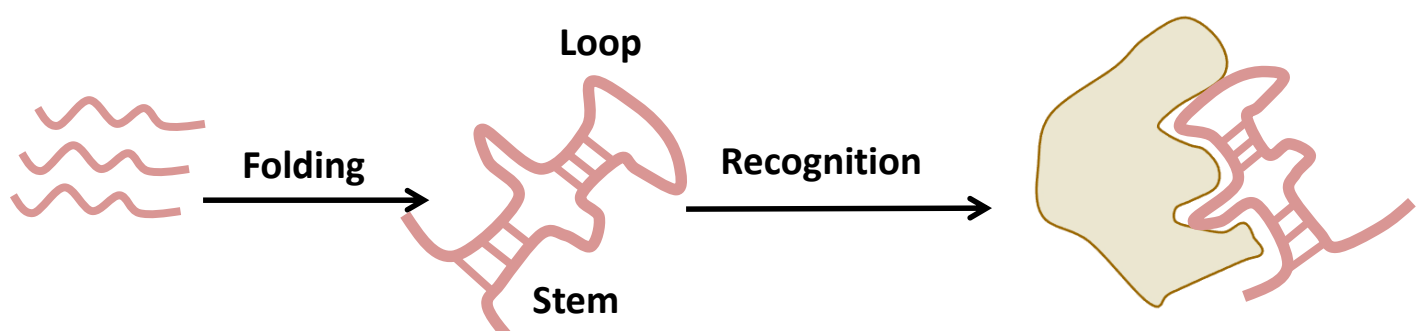

Figure 1.4: Schematic representation of aptamer functionality based on a complex 3D structure including stems and loops. The aptamer is interacting with a large target in this case.

Aptamers have been generated for a wide range of targets, with excellent specificity and with $K_{\mathrm{D}} \mathrm{S}$ ranging from mid-lower $\mu \mathrm{M}$ to $\mathrm{pM}$ concentration range. ${ }^{349110}$ Large targets, including proteins, provide multiple interaction sites (some targets interact with their aptamers in a molar ratio of 1:2 respectively) and low $K_{\mathrm{D}} \mathrm{S}$ are achieved. Despite the small molecular sizes and less interacting sites of some targets, many aptamers have been isolated with specificity and $K_{\mathrm{D}} \mathrm{S}$ in the low $\mathrm{nM}$ range such as the aptamers specific for $\mathrm{E}^{2723}$ and ethanol amine..$^{24}$

\section{Aptamers versus antibodies}

Although antibodies have been considered as the standard molecular probes for a long time (since the 1950s), aptamers provide the new emerging molecular recognition technology. They provide numerous practical and scientific advantages in comparison to antibodies. Their in vitro generation allows them to be generated in shorter time, for toxic targets and in theory for any given target. Aptamers can be generated to perform under elevated temperatures and $\mathrm{pHs}$ and in nonphysiological environments. They have higher resistance towards degradation, i.e., losing the ability to recognise the target via denaturing. Aptamers can be denatured and renatured in a reversible manner while maintaining their biological function. Once an aptamer is identified, it can be synthetically obtained in large quantities and easily modified with fluorescent dyes, linker groups (such as $\mathrm{SH}$ 
or $\mathrm{NH}_{2}$ ), electrochemical or Raman active groups, from commercial sources. Aptamers in general undergo conformational changes upon target binding which allow them to be successful biological reporters in biosensors. ${ }^{39-11}$ Their small size $(<10 \mathrm{kDa}$, and antibodies $\sim 155 \mathrm{kDa})$ makes them more applicable for electrochemical based transduction which is sensitive to binding events within the Debye length. ${ }^{25}$ The Debye length defines a screening distance of surface excess charges. It depends on the ionic strength of a solution and is mostly less than a nanometer in biological buffers. ${ }^{25}$

On the other hand, the in vivo generation (using a living species) of antibodies limits them to targets with no or minimal toxicity and adverse immune responses. The immunogenic generation of antibodies takes a long time and suffers from batch-to-batch variation. Antibodies are proteins that generally have large hydrophobic cores, thus they are subject to aggregation, degradation, and loss of biological function upon operation in elevated temperatures or non-physiological conditions. ${ }^{26}$ The typical size of antibodies vary from $10 \mathrm{~nm}$ to $15 \mathrm{~nm}$ indicating that recognition events occur outside the Debye length, where electrochemical based sensing takes place. ${ }^{25}$ Sensing with antibodies does not produce reportable (as in the case with aptamers) conformational changes and is mostly transduced via sandwich based assays where interaction with a secondary antibody is required. Examples of this type include the early pregnancy test using a lateral flow immune assay. ${ }^{27}$

\section{Therapeutic and biological application of aptamers}

Beside their potential use for sensing, aptamers have shown considerable promise in medical and pharmaceutical applications. Due to aptamers' high affinity and selectivity they can be used as drug delivery as well as therapeutic agents. ${ }^{28}$

\subsubsection{Aptamers in biosensors}

This section provides a brief literature review of different transduction techniques incorporating aptamers as the recognition elements. These techniques are optical, electrochemical, lateral flow, and size based transduction methodologies. The focus will be localised on the general schematic and construction components of the sensors, detection limits, limitations, advantages and disadvantages in comparison with other techniques. 


\section{Optical aptasensor (fluorescence: turn on and turn off mechanisms)}

Optical detection by fluorescence is one of the most popular signal transduction methods due to the availability of many fluorophores and quenchers and the ease with which aptamers can be modified for application in these systems. Recognition responses are translated based on quenching (giving a negative response) or enhancement (de-quenching and giving a positive response). However the systems can suffer due to the limitations of organic fluorophores including limited signalling ranges, high detection limit and self-quenching. ${ }^{29}$ Alternatively, QDs are inorganic semiconducting NPs that overcome the limitations encountered when using organic dyes. ${ }^{29}$

Chen et al. ${ }^{30}$ reported in solution optical sensing of Ochratoxin A (OTA), a common food contaminant. Fluorescein-labeled OTA aptamer was hybridised to a complementary DNA (cDNA) strand containing a quencher moiety, which brought the fluorophore and quencher into close proximity and quenched the fluorescence of the fluorescein as shown in Fig. 1.5a. Structural switching of OTA aptamer was triggered by the introduction of the target which released the cDNAquencher from the fluorescein-labeled aptamer. Based on this de-quenching mechanism, OTA was detected selectively (against structurally similar compounds) down to $1.9 \mathrm{nM}$ with a dynamic range from 2.4 to $247.6 \mathrm{nM}$.

Another approach of transforming aptamer signals by fluorescence is to modify the aptamer with a fluorophore and quencher at particular positions within the aptamer. Conformational changes induced by the target recognition bring the fluorophore and quencher into close proximity which terminates the fluorescence. This mechanism is represented by the work reported by Stojanovic et al. ${ }^{31}$ for the detection of cocaine, Fig. $1.5 \mathrm{~b}$. Cocaine was detected selectively down in the $\mu \mathrm{M}$ range and the sensing was also achieved in serum.

Instead of fluorescence turn off, Yamamoto and Kumar ${ }^{32}$ established a different molecularly engineered (RNA) aptamer biosensor for the detection of Tat protein of HIV. As shown in Fig. 1.5, the aptamer sequence was constructed in a molecular beacon-like, hairpin structure, and end labelled with a fluorophore and a quencher. When Tat was introduced, it disrupted the stem, releasing the fluorophore from the quencher and leading to an enhancement of the fluorescent signals, attaining qualitative detection.

Levy et al. ${ }^{29}$ used QDs (zinc sulfide shell with either cadmium selenide or cadmium telluride) for the detection of thrombin. As shown in Fig. 1.5d, thrombin 


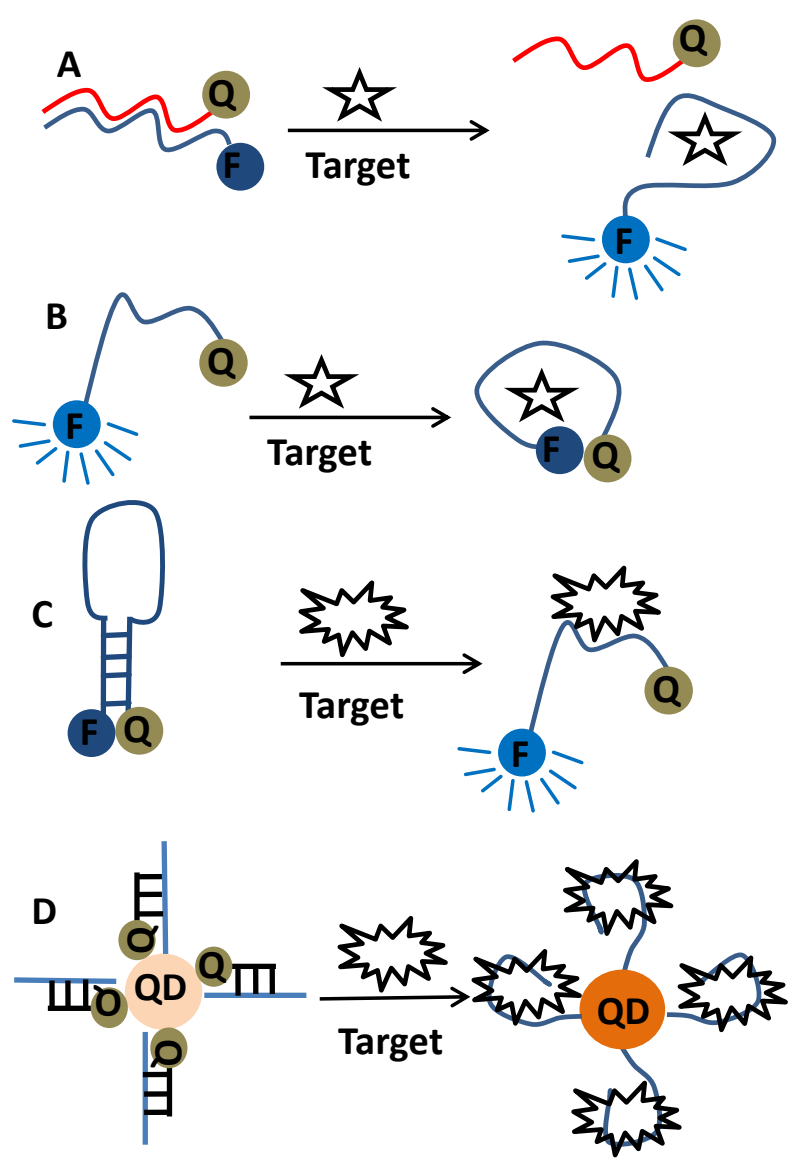

Figure 1.5: Schematic illustration of various sensing strategies employing aptamers and optical sensing based on fluorescence. a) A fluorescently labeled aptamer for OTA sensing based on the displacement of a quencher-labeled complementary DNA upon target recognition. $\frac{30}{b}$ ) Cocaine aptamer modified with a fluorophore and quencher, where recognition of the target brings the fluorophore and quencher into close proximity. ${ }^{31}$ c) Molecular beacon-like RNA aptamer labelled with a fluorophore and quencher for the detection of Tat protein based on the de-quenching mechanism. ${ }^{32} \mathrm{~d}$ ) QD aptasensor for thrombin detection, based on the displacement of a complementary DNA with a quencher previously hybridized to thrombin aptamer-linked to $\mathrm{QD} \cdot{ }^{29} \mathrm{~F}=$ fluorophore and $\mathrm{Q}=$ quencher. Stars represent small targets and starbursts represent large targets.

aptamer was linked to the QD through streptavidin-biotin interaction. A complementary DNA fragment containing a quencher was hybridised to the thrombin aptamer allowing energy transfer and quenching. When thrombin was introduced, the DNA fragment containing the quencher was released resulting in an increase of fluorescent intensity (signal turn-on). The sensor showed specificity when examined against nonspecific protein (Lysozyme).

Various aptamers have been engineered with fluorescence based detection and 
insightful mechanistic steps involved during the recognition were revealed. ${ }^{31} \mathrm{Si}$ multaneous detection of multi-target detection using aptamers-integrated with different fluorophores has been achieved. ${ }^{[33}$ However, alternative sensing schemes have to be developed to overcome a number of drawbacks. It is often difficult to design appropriate labelling strategies due to the lack of certainty of the target binding sites and accurate prediction of the conformational changes. Fluorescent labels have to be involved which is not always convenient for binding and could hinder the target recognition. The use of QDs involves some drawbacks such as the toxicity of heavy metals, decomposition, and aggregation.

\section{Optical aptasensor using nobel metal nanoparticles (absorption: aggregation and de-aggregation mechanisms)}

AuNPs have received tremendous attention in optical colorimetric biosensors due to their size and proximity dependent optical properties. Surface plasmon resonance in AuNPs (oscillations of the conduction electrons at the surface of NPs) occurs when the electric field of incident light displaces the electron cloud from equilibrium by excitation, the positive nuclei then act as a restorative force, $\underline{34}$ as shown in Fig. 1.6. Small particles are dominated by absorption effects whereas larger particles are dominated by scattering. Therefore, stabilised gold nanoparticles often exhibit somewhat sharp absorption peaks while aggregated particles are characteristically broad. The color of the colloidal gold solution (size range of 10-50 nm) changes from red (maximum peak absorption at $525 \pm 5 \mathrm{~nm}$ ) to purple/blue (appearance of a second band at $625 \pm 5 \mathrm{~nm}$ ) during AuNP aggregation. $\frac{35136}{}$ AuNPs have high extinction coefficient, over 1000 times higher than that of organic dyes, ${ }^{37}$ which makes them attractive signaling agents.

The key to a successful AuNP-based colorimetric sensor is the control of the colloidal AuNP dispersion and aggregation with analyte recognition. The stability of AuNPs depends on the net balance between interparticle attractive and repulsive forces. The most widely used method to prepare AuNPs is the citrate reduction method which produces AuNPs stabilized (against van der Waals attractioninduced aggregation) by the negatively charged citrate ions weakly adsorbed on the surface in water. 38,40

In 2004, Huixiang et al ${ }^{41 / 42}$ reported that ssDNA and dsDNA have different adsorption properties onto citrate capped AuNPs. Unlike dsDNA, ssDNA can nonspecifically adsorb onto the surface and stabilize the particles against salt induced aggregation. They were able to establish a colorimetric hybridization assay to de- 


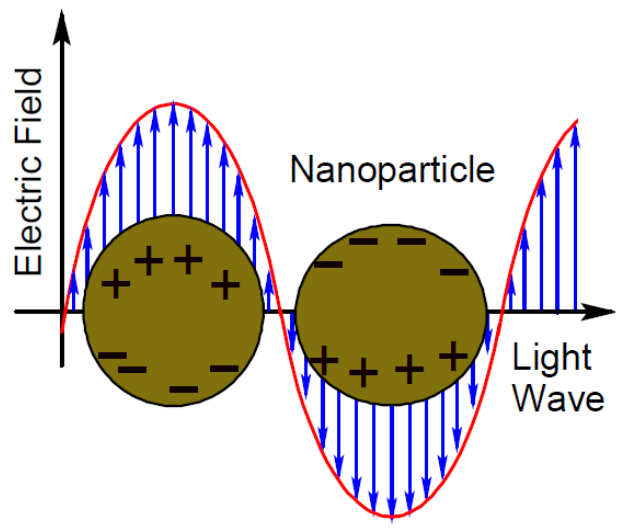

Figure 1.6: Surface plasmon resonance of AuNPs. Oscillation of the free conduction electrons in the AuNPs due to strong coupling with incident light.

tect DNA sequences down to the femtomole quantity as depicted in Fig. 1.7a.

Aptamers possess the ability to undergo a conformational change, induced by the target binding, from free uncoiled to folded structures. The integration of AuNPs and aptamer's target-induced conformational switch was the basis of many colorimetric aptasensors for small targets such as the detection of bisphenol A (LOD $=0.44 \mathrm{nM}), \stackrel{43}{\sqrt{3}}$ Potassium $(\mathrm{LOD}=1.7 \mathrm{mM}), \frac{5}{5}$ and Ochratoxin $\mathrm{A}(\mathrm{LOD}=20 \mathrm{nM})^{44}$ and large targets such as the detection of thrombin ${ }^{45}$ following the mechanism shown in Fig. 1.7p. In this scheme, the aptamer is non-specifically adsorbed on the surface of the citrate caped AuNPs via the the nitrogen bases of the DNA. The adsorption of the aptamer maintains the red color of the colloidal solution at reasonably high salt concentration. However, the target induced conformational change within the aptamer leads to desorption of the aptamer from the surface. Subsequently, AuNPs aggregate in response to the target turning the colloidal gold solution to purple/blue. The sensitivity of this assay type could be enhanced by optimising the concentration of the particles, the concentration of the aptamer, and the concentration of the salt. $\frac{5,43,45}{4}$

The target analyte can act as a cross-linking agent that will trigger aggregation. Thrombin aptamer interacts with the target in a molar ratio of 1:2 respectively. $\underline{46}$ After immobilisation of the aptamer on AuNPs through thiol-Au bonds, the addition of the target induced AuNP-aptamer aggregation (Fig. 1.7k) enabling the detection of thrombin as low as $5 \mathrm{pM} . \underline{46}$

Aggregated AuNPs, i.e. cross-linked by an aptamer, can be driven towards dispersion upon target recognition. Liu et al. ${ }^{47}$ developed a method to detect adeno- 
A
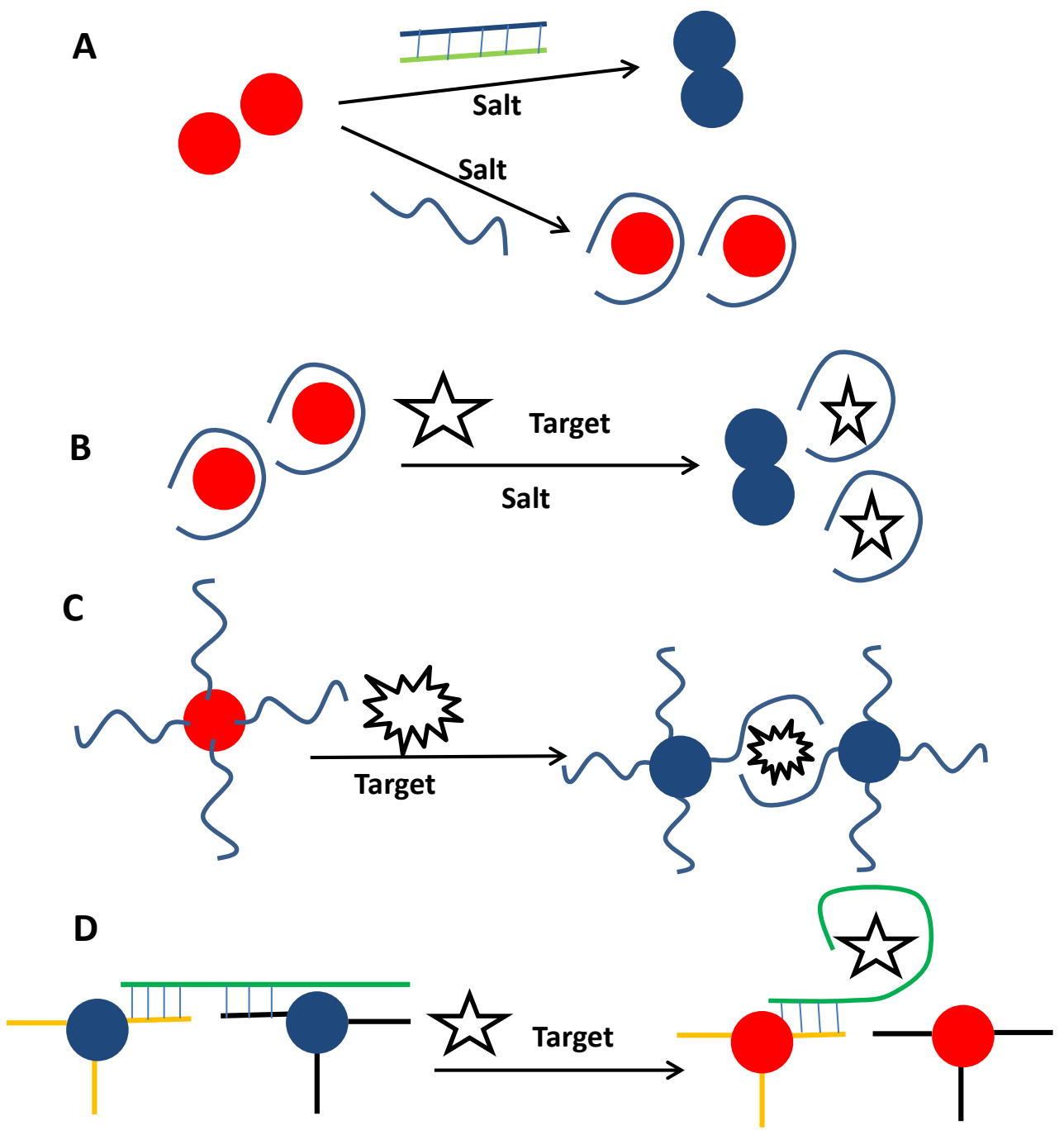

Figure 1.7: Schematic illustration of various sensing strategies employing AuNPs for colorimetric sensing. a) Sensing DNA based on the different adsorption properties of ssDNA and dsDNA. ${ }^{42} b$ ) Target-induced conformational change which is the basis of a number of colorimetric sensors for small targets including Ochratoxin A. ${ }^{\sqrt{44}} \mathrm{c}$ ) Thrombin causes the aggregation of AuNP-aptamer by interacting with the aptamer in a molar ratio of 1:2 respectively ${ }^{46} \mathrm{~d}$ ) Adenosine aptamer (green line) used to hybridise and cross-link two complementary sequences (orange and black lines) coupled to AuNPs. Recognition of adenosine releases the complementary sequence adjacent to the binding cavity and breaks down the aggregates. ${ }^{47}$ Red circles $=$ monodispersed AuNPs and blue circles = aggregated AuNPs. Stars represent small targets and starbursts represent large targets.

sine triphosphate (ATP) starting from aggregated AuNPs. In Fig. 1.7 d, the ATP aptamer was extended at the $5^{\prime}$ end and was cross-linked to two complementary sequences (black and orange lines) previously coupled to AuNPs (via thiol-Au chemistry), thus producing blue colored suspension of aggregated AuNPs. When ATP was introduced at $\mathrm{mM}$ concentration, the aptamer (green line) folded on the 
target causing the complementary sequence adjacent to the binding pocket (black line) to dissociate at room temperature. The target concentration was monitored quantitatively according to the change in absorbance ratio (absorption at $522 \mathrm{~nm}$ / absorption at $700 \mathrm{~nm}$ ).

Overall, AuNP based colorimetric aptasensing is considered to be rapid, simple, sensitive and cost effective approach to detect small and large targets. The examples discussed here outline the detection in water-based samples (buffered solutions or environmental samples). Detecting in complex samples has yet to be demonstrated (such as detection in urine or blood). Major drawbacks of the AuNP based colorimetric sensing include lack of operation in non-transparent samples, and vulnerability to non-specific aggregation induced by high ionic strength samples. With regard to AuNP based colorimetric assay for small molecules, signal transduction depends on the dissociation of the aptamer from the surface. Previous studies included various aptamers with different lengthes. Excess bases, outside the target binding pocket, may be innocent with respect to target binding affinities, $\stackrel{48 / 49}{ }$ their effect on signal transduction is expected to depend on the sensor format used. Further investigation of the length of aptamer sequences on AuNP based colorimetric assay should be considered.

\section{Lateral flow assay (LFA)}

The lateral flow Aptasensor (LFA), also called a dipstick, is among the vast growing strategies for qualitative (yes/no type assay) and occasionally quantitative analysis. LFA's simplicity and instantaneous results promote it to fulfill the demands for point of care and on-site testing. LFAs unparalleled successes in the qualitative pregnancy and sugar immune-sensors make them potentially adaptable to the detection of other targets. $.50+52$

The signal transduction is based on a combination of chromatography (separation of different components based on differences in their movement through a porous membrane) and antigen-antibody properties (or aptamer target interaction). LFA conventionally comprises of four parts - sample pad, conjugate pad, test pad (containing test and control zones) and absorbent pad as depicted in Fig. 1.8. ${ }^{53}$ AuNPs are the most widely used signal source in LFA due to their high extension coefficient and ease of functionalisation. 54

The targeted analyte in a liquid solution is introduced to one end of the test strip and migrated to the other end of the strip by capillary force. Large targets can 


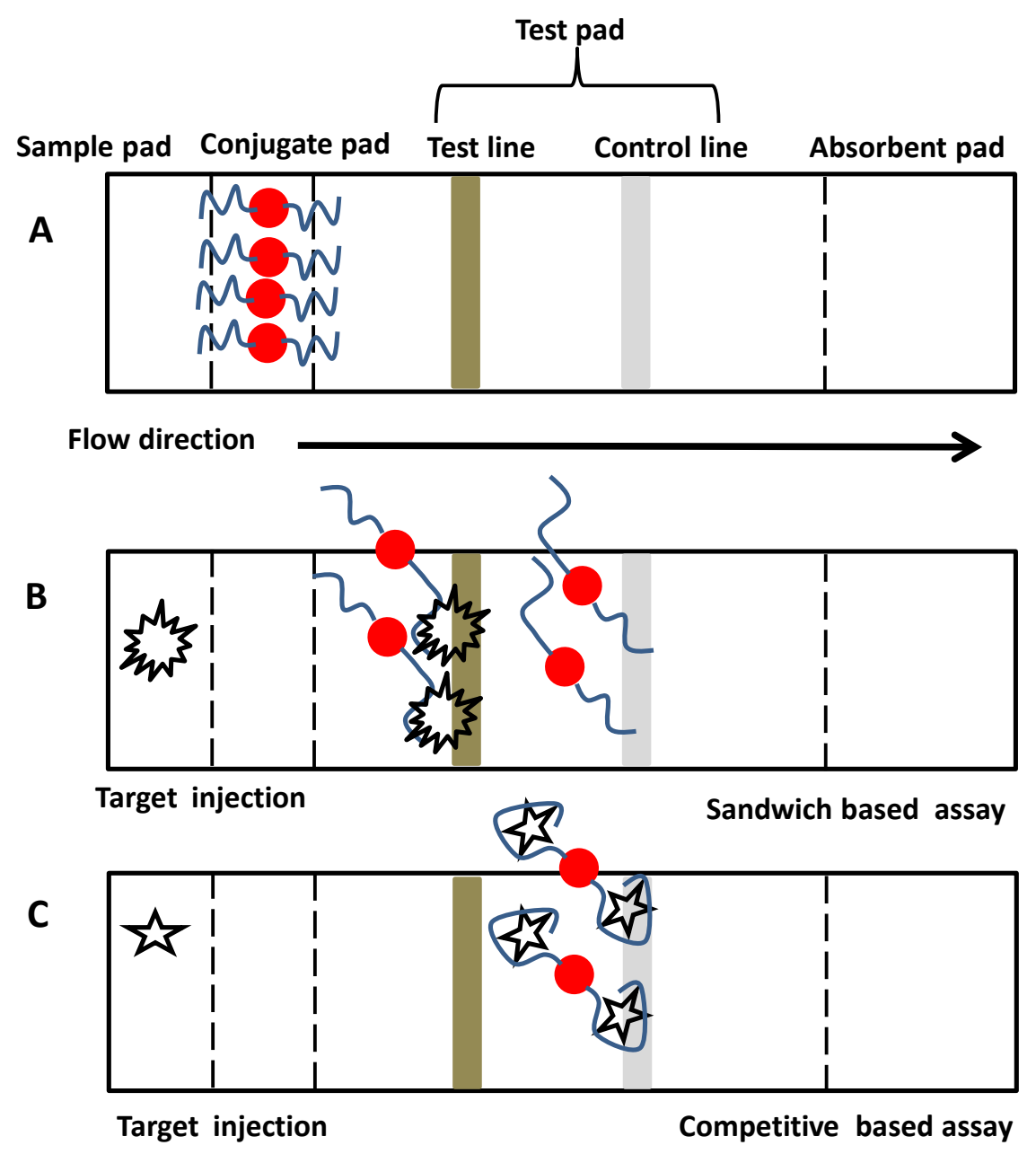

Figure 1.8: Schematic illustration of lateral flow assay. a) Different parts comprising the test strip. b) Sandwich assay type where the presence of the target is indicated by the formation of the test line (resulting from target mediated hybridisation between the recognition element on the particles and the recognition element on the strip). c) Competitive assay type where the presence of the target is indicated by the disappearance of the test line. The control line is formed regardless of the presence or absence of the target. Stars represent small targets and starbursts represent large targets.

be detected based on a sandwich type assay because of their ability to bind two probes immobilised on the test zone and AuNPs, as shown in Fig. 1.8p. Examples of this type includes the detection of thrombin with a limit of detection down to $2.5 \mathrm{nM}$. Competitive assay format is more suited for targets with low molecular weight as depicted in Fig. 1.8c. Ochratoxin A (OTA) was detected by a competitive assay where the test and control lines are complementary sequences to the core region (target binding pocket) and $3^{\prime}$ end of OTA aptamer. ${ }^{[55}$ The presence of OTA, as low as $2.5 \mathrm{nM}$, induced a conformational change making the core region unavailable for the test line while the availability of the $3^{\prime}$ end to the control line is not effected. 


\section{Electrochemical aptasensor}

Electrochemical based sensing presents some advantages compared to other transduction methods especially readability of signal transduction, high sensitivity, speed and accuracy, and easily integrated with aptamers and nanotechnology. The biological event can be transduced by either labeling the aptamer with a redox active labels (e.g. ferrocene or methylene blue) or by label free methods when monitoring the kinetics of a redox active species diffuse in the solution (e.g. ferrocyanide/ferricyanide redox couple) $\cdot{ }^{56-58}$ Electrochemical cells with three electrode configuration are frequently used. A gold electrode (or a modified form) is usually used as the working electrode and hybridised with recognition elements. Electrochemical signals generated from the recognition interactions of aptamers and their targets can be monitored using amperometric, potentiometric, and impedimetric techniques. ${ }^{59-62}$ Selected examples of the construction of electrochemical aptasensors towards the analysis of some targets will be reviewed in this section.

Ultra-sensitive detection of a small molecule OTA ${ }^{63}$ was accomplished by labelling the OTA aptamer with, an electrochemically active label, methylene blue (MB) and immobilising on gold electrode using thiol-gold coupling. As shown in Fig. 1.9 a, in the absence of the target OTA, the aptamer brings MB into close proximity to the electrode surface resulting in a maximum current (detected using square wave voltammetry). Upon introduction of the target, the aptamer undergoes a conformational change, placing the MB away from the electrode and minimising the electron transfer, signal off based sensing. OTA was detected down to the $0.25 \mathrm{pM}$ in wine samples. Xiao et al. ${ }^{64}$ reported on the detection of thrombin in blood serum using the same strategy.

On the other hand, the structural change in the aptamer upon target recognition could produce a conformation that enables enhanced electron transfer. Thrombin aptamer was modified with ferrocene (FC) label and self assembled on gold electrode via thiol-gold bonds. The aptamers switched their conformation from random coil-like configuration to the quadruplex structure upon thrombin recognition as depicted in Fig. 1.9p. Thrombin was detected as low as $0.5 \mathrm{nM}$ and up to $35 \mathrm{nM}$ (using differential pulse voltammetry). The sensor could be regenerated up to 25 times with no loss of sensitivity. ${ }^{\sqrt{65}}$ The same strategy was adopted for the detection potassium ions in the $\mathrm{mM}$ range by the same group. 66

Electrochemical impedance spectroscopy (EIS), discussed in depth in Chapter 4 , is an alternating current $(\mathrm{AC})$ method that measures electrochemical components 
A
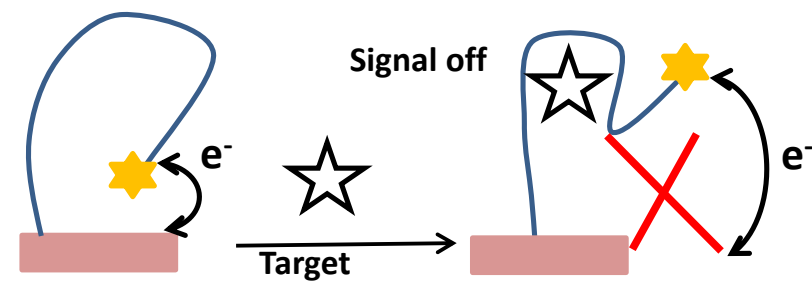

B
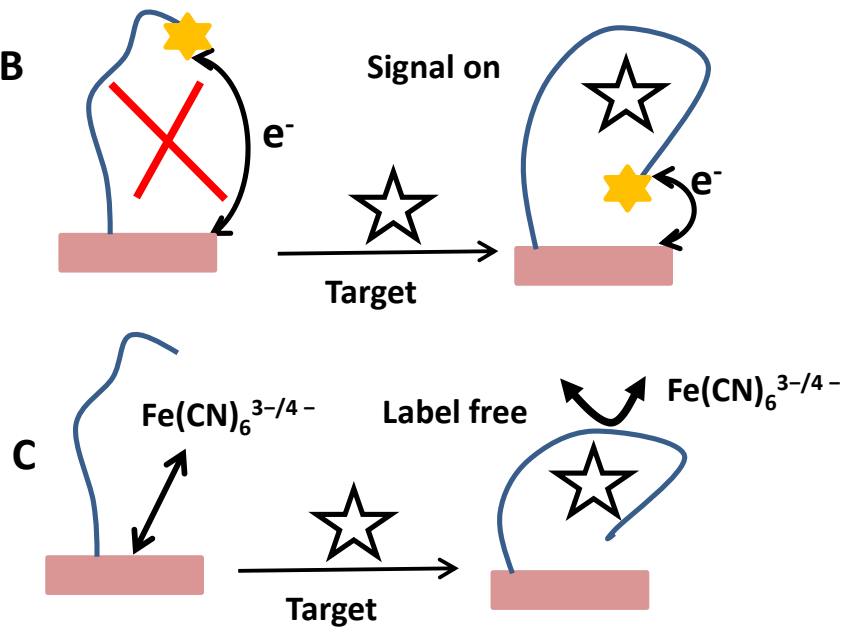

D

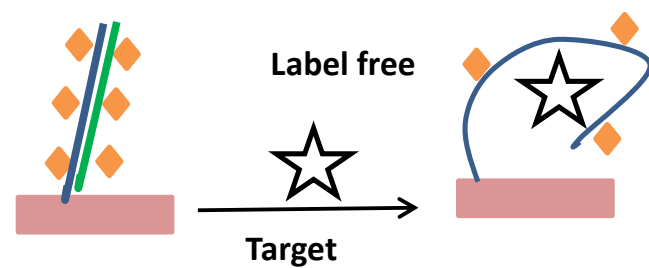

Figure 1.9: Schematic illustration of various electrochemical sensing strategies. a) Signal off approach for the detection of a target is based on the displacement of a redox active label away from the electrode (orange $3^{\prime}$ end tag). b) Signal on approach. The recognition of the target induces a conformation that brings the redox label into close proximity and enhances the electron transfer. c) Label free approach where target recognition induces a more compacted structure of the aptamer that limits the accessability of the redox couple $\mathrm{Fe}(\mathrm{CN})_{6}{ }^{3-/ 4-}$ to the electrode surface. d) Label free approach where the recognition of the target induces the release of a complementary DNA strand with the adsorbed $[\mathrm{Ru}(\mathrm{NH}$ $\left.\left.{ }_{3}\right)_{6}\right]^{3+}$ molecules (orange rhombus). Stars represent small targets.

(including biological interactions) that impede the charge transfer resistance $\left(R_{C T}\right)$ of a redox reaction at the working electrode. EIS was used to establish a label-free electrochemical aptasensor for the detection of OTA. ${ }^{67}$ The OTA specific aptamer was tethered to an $\mathrm{Au}$ electrode and the redox probe ions, $\mathrm{Fe}(\mathrm{CN})_{6}{ }^{3-/ 4-}$, were diffused in the detection solution. Upon exposure of the modified Au electrode to OTA, the aptamer-OTA complex was formed resulting in a new configuration that hinders the accessibility of the redox prob to the electrode surface as shown in Fig. 1.9k. Consequently, the $\mathrm{R}_{\mathrm{CT}}$ increased linearly as the concentration of OTA 
increased in the range of 0.12 to $0.40 \mathrm{nM}$. Other examples that used the same sensing format include the detections of $\mathrm{E} 2{ }^{68}$ and chloramphenicol. $\frac{69}{6}$

Label-free electrochemical aptasensing can also be established by monitoring the dissociation of a complementary sequence upon recognition. Shen et al. ${ }^{70}$ fabricated an electrochemical aptasensor for the detection of adenosine using a redox mediator, $\left[\mathrm{Ru}\left(\mathrm{NH}_{3}\right)_{6}\right]^{3+}$. RuHex was used as an electroactive complex which was the key aspect of the sensor transduction mechanism. RuHex can bind electrostatically to the anionic phosphate groups at the backbone of DNA. RuHex has a well-defined peak in cyclic voltammetry $(\mathrm{CV})$ at $0.2 \mathrm{~V}$ and is a characteristic signature reflecting the amount of DNA strands coupled to the electrode surface. When adenosine aptamer modified electrode was exposed to the target, the short complementary DNAs were released together with the associated RuHex, shown in Fig. $1.9 \mathrm{~d}$, leading to lower electrochemical signals. Adenosine was detected from 0.1 to $1000 \mu \mathrm{M}$. It should be noted that the release of the complementary sequence upon target detection can additionally be monitored by CV or EIS with no need for the weakly adsorbed RuHex molecules as reported for the detection of bisphenol $\mathrm{A}^{71}$ and adenosine, respectively. $\underline{72}$

Overall, electrochemical based aptasensing measurements are vast and can provide extremely low detection limits, for example attomolar detection limit has been reported for thrombin. ${ }^{71}$ The integration of electrode surfaces with different nanomaterials (such as AuNPs, QDs, carbon nanotubes, or nanostructured conducting polymers) provides electrodes with a larger surface area, improved surface reactivity, means of aptamer tethering, and rapid electron transfer. These properties provide electrochemical aptasensor with adequate capability for application of "real-world" samples with no need for extensive sample pretreatment processes. However, laborious passivation steps are usually required with goldbased electrodes to eliminate non-specific adsorption of the target or unknown sample composition.

\section{Size aptasensor (aggregation and other size based formats)}

As mentioned previously, target induced conformational changes within the aptamer molecules can be utilised in various sensing platforms. Small molecules, such as hormones, adenosine and cocaine, interact within the aptamer binding pockets. Since these molecules are structurally less complex, they provide single site binding (1:1 aptamer : target molar ratio). ${ }^{59}$ In contrast, proteins, and other larger targets are structurally more complex and could provide various sites inter- 
acting with the aptamer molecules through hydrogen bonds, electrostatic interactions and shape complementarity, therefore dual-site binding is achieved (2:1 aptamer : target molar ratio). $\frac{59}{5}$ The latter is the key aspect of constructing size based aptasensors where target binding can be used to cross-link and aggregate a monodisperse NP population. There are other aptasensor formats that utilise the size changes occurring on NP-aptamer conjugates induced by recognition of specific targets. This section aims to provide examples how the change in size of NP-aptamer conjugates can be used as a means of transduction of the binding events.

Yang et al. ${ }^{73}$ provided the first aptasensor that used Dynamic Light Scattering (DLS) in the signal registration and the detection of adenosine. Although adenosine is a small molecule that provides a single site interaction with the aptamer, it was detected using an aggregation type assay by the use of a special molecular engineering format. In the engineering of this sensor, the adenosine aptamer was split into two ssDNA fragments and both hybridised to different AuNP populations through a Au-thiol interaction. The presence of adenosine brought the two types of AuNPs together causing the formation of AuNP aggregates as illustrated in Fig. 1.10a. The change in the average particle size by DLS can be monitored as the recognition of adenosine occurs. The average size of AuNPs increased accordingly as the concentration of adenosine increased. It was found that adenosine could be detected as low as $7 \mathrm{nM}$ with a logarithmic response up to $80 \mu \mathrm{M}$. The method showed good specificity when uridine, cytidine, and guanosine were examined.

DLS was also used as the readout technique in the patented work of Wanekaya et al. ${ }^{74}$ for the direct detection of thrombin, a molecule with a hydrodynamic diameter of $4 \mathrm{~nm} \cdot{ }^{76}$ As shown in Fig. 1.10 , the thrombin aptamer was hybridised to AuNPs (12.7 nm) and then exposed to serial dilutions of thrombin (1.4 to 47 $\mathrm{nM}$ ). It was discovered that DLS is able to detect the increase in size of AuNPaptamer conjugates. It should be noted that the increase in the size is a result of the formation of aptamer-thrombin complex and is not a result of NP aggregation.

Platt et al. $\frac{75}{75}$ constructed a novel aptasensor for the detection of platelet derived growth factor (PDGF). PDGF interacts with its aptamer in a molar ratio of 1:2 respectively, allowing the design of aggregation assays. Magnetic rod shaped Au-Ni particles were hybridised with PDGF aptamer. Tuneable resistive pulse sensing (TRPS) that can measure the size and speed of NPs traversing through a nanopore was used for signal readout (TRPS will be discussed in detail in Chapter 2 ). The size and speed were used to characterise the aggregates from the recogni- 
A
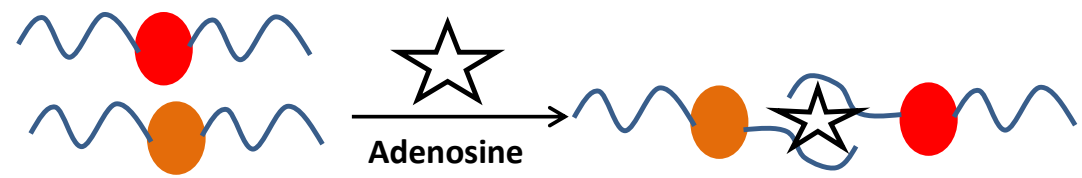

$B \sim$

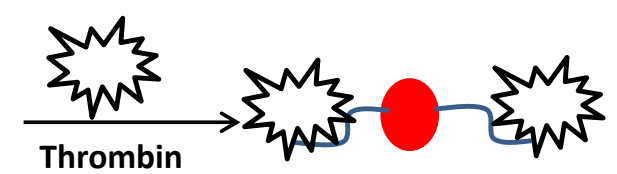

$c \sim \sim \sim \sim c$
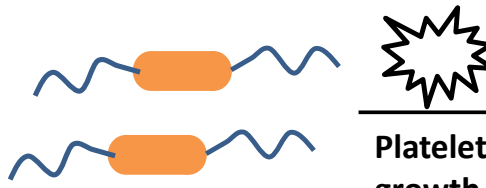

Platelet derived

growth factor

Figure 1.10: Schematic illustration of various size based sensing strategies. a) Detection of adenosine using split aptamer fragments tethered to different AuNP populations, and aggregation caused by adenosine recognition. ${ }^{73} \mathrm{~b}$ ) Thrombin size aptasensor, recognition of thrombin caused an increase in the average hydrodynamic size of the hybridised AuNPs. ${ }^{74} \mathrm{c}$ ) Detection of platelet derived growth factor (PDGF) using aptamer-modified rod shaped Au-Ni NPs and oriented aggregation assay. ${ }^{75}$ Stars represent small targets and starbursts represent large targets.

tion of PDGF down to the $10 \mathrm{fM}$ concentration, as shown in Fig. 1.10k. The sensor showed excellent specificity against non-binding protein, bovine serum albumin (BSA).

\section{Aptasensors overview}

Thus far, various types of aptasensor have been developed and the frequently reported methods for the detection of small molecular targets were reviewed in this chapter. The same 10-15 aptamers were always used to develop new sensing platforms, despite the hundreds of aptamers currently available. These aptasensors were classified on the basis of the detection technique; thus optical aptasensors, lateral flow based aptasensors, as well as electrochemical aptasensors or size based aptasensors. The aforementioned detection methods have their advantages and limitations; thus, it is the specific application of the aptasensor that determines the best method to use. It should be noted that this chapter does not attempt to provide a comprehensive survey of the different aptasensor transduction methods, its objective was, instead, to give a general overview and to provide general examples in the field. 


\section{Challenges and opportunities}

With specific regard to aptasensing for small molecules, it is difficult to design appropriate and efficient labelling strategies, whether with fluorescence or with electrochemical sensors, due to the lack of certainty of the target binding sites and accurate prediction of the conformational changes in the aptamer molecules. Additionally, the labelling process makes the experiments more complex and could affect the binding affinity between the aptamers and their targets.

Furthermore, small targets including ochratoxin $\mathrm{A},{ }^{77}$ cocaine, ${ }^{31}$ and theophlline ${ }^{78}$ do not have complicated 3D structures (they have planar structures). This class of targets are recognised by incorporation within the binding pockets of their aptamers. Size based sensor triggered by a target cross-linking an aptamer tethered to nanoparticles is not expected (unless special aptamer design is constructed, as the rare case of split adenosine aptamer ${ }^{\sqrt{73}}$ ). Therefore, there is a clear motivation to investigate the possibility to develop a label free size based aptasensor that realises on the general property that small molecule binding aptamers adopt a more compact folded structure when they bind to their target.

SELEX process selects aptamers for affinity, elimination of redundancies within aptamer sequences that do not affect affinity is not part of the process. The effect of non-binding segments of the aptamers on signal transduction has yet to be demonstrated, particularly with sensors depending on aptamer dissociating from surfaces (i.e., colorimetric based assays). Additionally, comprehensive understanding regarding the effect of long and truncated aptamer sequences on signal transduction could be attained by comparing the performance of the same aptamer across a number of aptasensors with distinct transduction mechanisms.

Label free electrochemical based aptasensors by far are the most successful platforms reporting ultrasensitive detection limits. They commonly feature aptamer probes covalently tethered to gold electrodes where target-induced conformational changes can be monitored electrochemically. Non-specific adsorption of aptamers is perceived to be undesirable in electrochemical sensors, however, it has yet to be explored as an alternative sensing strategy.

These challenges and opportunities inform the thesis research objectives elucidated in Section 1.2.2. 


\subsection{Research problems and objectives}

The aptasensors that will be developed in this thesis are targeting a naturally occurring estrogenic compound, 17- $\beta$ estradiol (E2), that also occurs as an environmental contaminants. E2 was previously detected in the $\mathrm{pM}$ concentration range in commercial dairy products $(6.6 \mathrm{pM})^{79}$ and in urine samples $(100 \mathrm{pM}) . \stackrel{68 / 80}{\mathrm{E} 2}$ was also detected in natural water samples at the nM concentration levels (60-377 $\mathrm{nM}) . \underline{81.83}$ Thus, target concentrations for sensor operation depend on the application, however, quantitation in the $\mathrm{pM}$ range is generally desirable. The following section focuses on providing a background of some naturally occurring and synthetic estrogenic compounds and highlights the challenges encountered in determining this class of molecules.

\subsubsection{Estrogenic derivatives}

Environmental contaminants are an ever growing issue around the world. In recent years, great attention has been directed to the potential impact of a class of chemicals that disrupt the normal functions of the endocrine system in humans and wildlife in agonist or antagonist manner. This class of molecules is known as Endocrine Disrupting Compounds (EDCs) and was defined by the United States Environmental Protection Agency (USEPA) as "an exogenous agent that interferes with the production, release, transport, metabolism, binding, action, or elimination of natural hormones in the body responsible for the maintenance of homeostasis and the regulation of developmental processes". .84

Scheme1.1 shows examples of naturally occurring and synthetic estrogenic chemical structures. It should be noted that natural estrogens are indigenous hormones, including estrone (E1), 17- $\beta$ estradiol (E2), and $17 \alpha$-estradiol (E2-17 $\alpha$ ), that present at critical levels. $\frac{85}{6}$ However, external exposure to these compounds promotes them to be endocrine disrupters. ${ }^{[85}$ There are many non-estrogenic chemicals that are classified as EDCs ${ }^{\sqrt{86}}$ and beyond the scope of this thesis. The major emission source of natural estrogenic compounds is farm animals. However, man-made sources such as waste water treatment plants and domestic effluents are secondary main sources of estrogenic compounds in water ways. $\stackrel{87}{ }$ Several reports found these molecules at levels ranging from few $\mathrm{nM}$ up to $\mu \mathrm{M}$ concentrations in rivers and water ways in New Zealand ${ }^{81}$ and worldwide. ${ }^{82}$ Estrogenic compounds have very low water solubility $0.3-13 \mathrm{mg} / \mathrm{L}$, low vapour pressures, relatively high pKa values (above 10) indicating non-volatility, and lipophilic 
characteristics that might enable their adsorption on solids and environmental and biological matrices. $\frac{88}{8}$
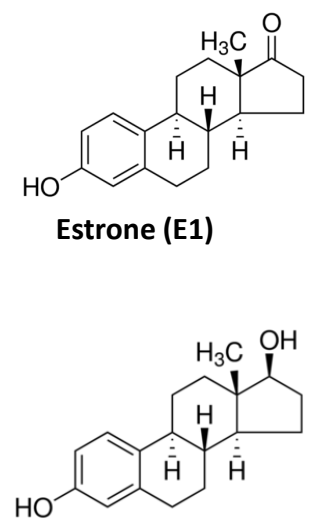

17- $\beta$ estradiol (E2)

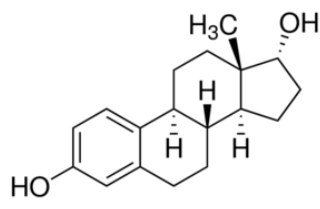

17- $\alpha$ estradiol (E2-17/ $\alpha$ )

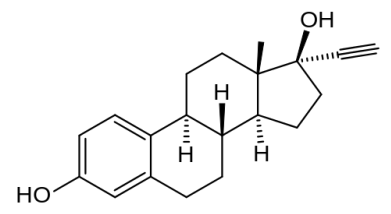

$17 \alpha$-Ethynylestradiol (EE2)

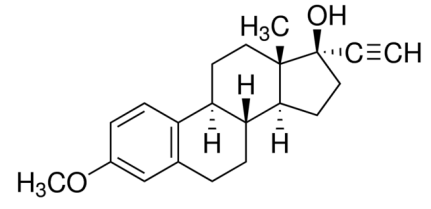

Mestranol (MeEE2)

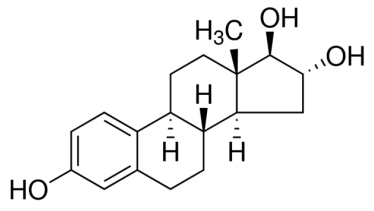

Estriol (E3)

Scheme 1.1: Chemical structures of the abundant estrogenic EDCs in the environment.

Environmental and biological monitoring of the concentration of estrogenic compounds is a difficult analytical task, because they are present at very low concentrations and in complex matrixes (including natural water and urine). Thus, in general, complicated, time consuming extraction and purification processes (using solid phase or liquid-liquid extraction) are usually employed prior to their analysis. Estrogenic EDCs are generally detected using immunoassay with radioactive or fluorescent labelling, ${ }^{89}$ high-performance liquid chromatography mass spectrometry (HPLC-MS), or gas chromatography mass spectrometer (GC-MS) ${ }^{90}$ with high sensitivity down to the range of $\mu \mathrm{M}-\mathrm{pM}$ concentrations.

The majority of estrogenic EDCs belong to the same steroidal family, having three six member rings and a single five member ring as the structural framework, and exhibiting very similar chemical behaviour. This gives rise to the selectivity problems (cross-reactivity of one of the members with the others) encountered in the conventional EDC analytical methods (HPLC and GC-MS). ${ }^{89.90}$

Despite the excellent sensitivity of HPLC and GC-MS, time consuming preconcentration and matrix purification processes are required making the monitoring of estrogenic EDCs impractical exercise and cost-ineffective. ${ }^{82}$ Immun- 
sensors suffer from several issues including immunogenic generation of the antibodies making their generation for toxic targets unachievable, degradation of the antibody, batch-to-batch variations, the requirement of radioactive or fluorescent labels for signal transduction which are not favoured for field screening and regular monitoring of estrogenic EDCs. ${ }^{87}$

\subsubsection{Research objectives and thesis overview}

The principle aim of this project was to design, develop, characterise and apply various alternative sensing platforms towards $17-\beta$ estradiol (E2) making use of a previously isolated 75-mer ssDNA aptamer (by Dr. Shalen Kumar and Professor Kenneth P. McNatty, reproductive biology lab, School of Biological Sciences at VUW $\left.{ }^{23}\right)$.

- Tunable Resistive Pulse Sensing (TRPS) and Dynamic Light Scattering (DLS) are techniques used to characterise the size and surface potential ( $\zeta$-potential) of nanoparticles (NPs) suspensions. Aptamers have been widely employed as bio-receptors based on their conformational change induced by the target recognition. Therefore, the first goal of this project was to investigate the use of TRPS and DLS to probe the change in size and $\zeta$-potential of NPs coupled to the aptamer upon recognition of E2. To overcome the limitations of TRPS and DLS, a well-integrated carboxylated polystyrene NPs was used to tether the aptamer through an amide bond and enable registering binding events when the aptamer adopts a more tightly folded conformation around the target (Chapter2).

- Gold nanoparticles (AuNPs) have been extensively used in biosensor applications due to their unusual size and proximity dependent optical properties. They can provide simple means to detect targets colorimetrically. The second objective of this thesis was to develop a colorimetric aptasensor for E2 as well as to understand the effect of the aptamer length on the signal transduction. Chapter 3 describes a thorough characterization of the necessary and unnecessary nucleotides needed for developing a colorimetric AuNP based aptsensor for E2. The effects of deleting excess flanking nucleotides (non-binding) from the 75-mer aptamer enabled significantly enhanced sensitivity for the target molecule.

- Electrochemical impedance spectroscopy (EIS) is particularly well-suited to probe the binding events on an electrode surface as well as character- 
ising the surface modifications. The third objective of this thesis was to develop a sensitive EIS based sensor for E2. Sensing E2 using EIS (Chapter 4) started with employing the 75-mer aptamer on a conducting polymer (poly-pyrrole-pyrroleacrylic acid) based electrode to achieve one fM level of detection in buffered solutions. The work was accomplished in conjunction with Bicheng Zhu, a PhD student with Professor Jadranka Travas-Sejdic, School of Chemical Sciences, University of Auckland.

As an alternative, the non-specific adsorption of aptamer onto Au surfaces was exploited to develop a novel EIS based sensing platform to detect E2. Au based electrodes bearing non-specifically adsorbed aptamers were found to be fundamentally different from the electrodes conventionally tethered with aptamers through thiol-linkers. The non-specifically adsorbed aptamer was found to completely coat the surface to passivate it against spurious interactions, as well as cross-linking the AuNP coated electrodes. This combination of properties resulted in a simply fabricated and highly effective sensor for E2, whereby an increase in the EIS signals is resolved when targetbound aptamers dissociate from the electrode surface. The sensor exhibited a fM level of detection, excellent selectivity against potentially interfering molecules, and operation in urine samples.

- By applying the same aptamer to different sensor formats, the advantages and disadvantages of each signal transduction mechanism were compared (Chapter 5). The differences between the performance of long aptamer sequences and truncated versions and their impact on various sensing schemes was investigated. 


\section{Chapter 2}

\section{Size based aptasensor}

\subsection{Abstract}

This chapter presents a new sensor design that exploits aptamer functionalized NPs to transduce the signal of aptamer receptors binding to E2. A previously isolated 75-mer aptamer capable of binding E2 with a $K_{\mathrm{D}}$ of $25 \mathrm{nM}$ was tethered to the surface of carboxylated polystyrene NPs. ${ }^{23}$ Upon exposing the aptamer functionalized NPs to E2 in buffered solution, tunable resistive pulse sensing (TRPS) and dynamic light scattering (DLS) were used to observe a distinct reduction of the conjugated particle size, reduced duration time (full width at half maximum (FWHM)) in TRPS, and a less negative $\zeta$-potential by DLS, which can be correlated to the $\mathrm{E} 2$ concentration in the lower $\mathrm{nM}$ range. The sensor showed similar affinity towards other hormones of the E2 steroidal family and excellent discrimination against potential non-steroidal interfering agents. The simplicity of the sensing scheme makes it readily applicable to other low molecular weight targets, as it was further demonstrated using a known adenosine aptamer. ${ }^{48}$ In addition to sensing, the developed method could potentially be used to guide the synthetic evolution of aptamers to attain better binding affinity and specificity.

\subsection{Introduction}

The relatively small size of aptamers (generally on the order of 20-100 nucleotides long, corresponding to fully extended lengths of 6.8-34 nm) compared with other 
recognition elements such as antibodies opens up the possibility of simple new physical means of signal transduction. ${ }^{9192}$ The ability of aptamers to recognize small molecular targets including hormones and narcotics is linked to the aptamers' propensity to adopt a folded three-dimensional conformation that provides a specific binding pocket for the target. ${ }^{993}$ Signals can then be transduced from the target-induced conformational switching via mechanisms that are not readily available to conventional antibody-based receptors or for aptamers targeting macromolecules that are larger than the aptamer. For example, and as detailed in Chapter 1.1.3. when pairs of fluorescent dyes are attached to different sections of the aptamer, binding the target may bring the dyes into close enough proximity to result in detection via fluorescent resonant energy transfer. ${ }^{[31}$ Alternatively, a redox probe can be attached to the distal end of an aptamer immobilized on an electrode surface such that binding the target brings the redox probe within electron transfer distance of the electrode and a current is measured. 94 While these methods demonstrate the ability to detect binding induced changes in aptamer conformation with high sensitivity, their successful implementation in a given system requires detailed knowledge of specific aptamer conformations and target interaction sites, as well as more design complexity, in order to engineer strong coupling between the binding event and signal from the attached probes.

Herein, a novel scheme is reported that explores a simple alternative means to detect the binding of a small molecule in solution via measuring the contraction in aptamer size that generally accompanies the binding of a small molecule. It should be noted that the scheme does not require knowledge of the specific conformation of an aptamer-target complex. The key to this approach, depicted in Scheme 2.1, is to tether the aptamers to the surface of NPs and resolve the changing size of aptamer functionalized particles when target molecules induce a more tightly-folded aptamer conformation on the particle surface. The success of this strategy is ensured by the availability of functionalizable NPs of a suitable size and excellent monodispersity combined with the modern solution-based particle sizing techniques to resolve diameters with better resolution than the binding induced changes in average aptamer size. Other size based aptasensor utilised the increase in size of the conjugate upon target recognition using DLS ${ }^{74}$ or aggregate formation using DLS ${ }^{73}$ and TRPS ${ }^{75}$ as the signal transduction. 


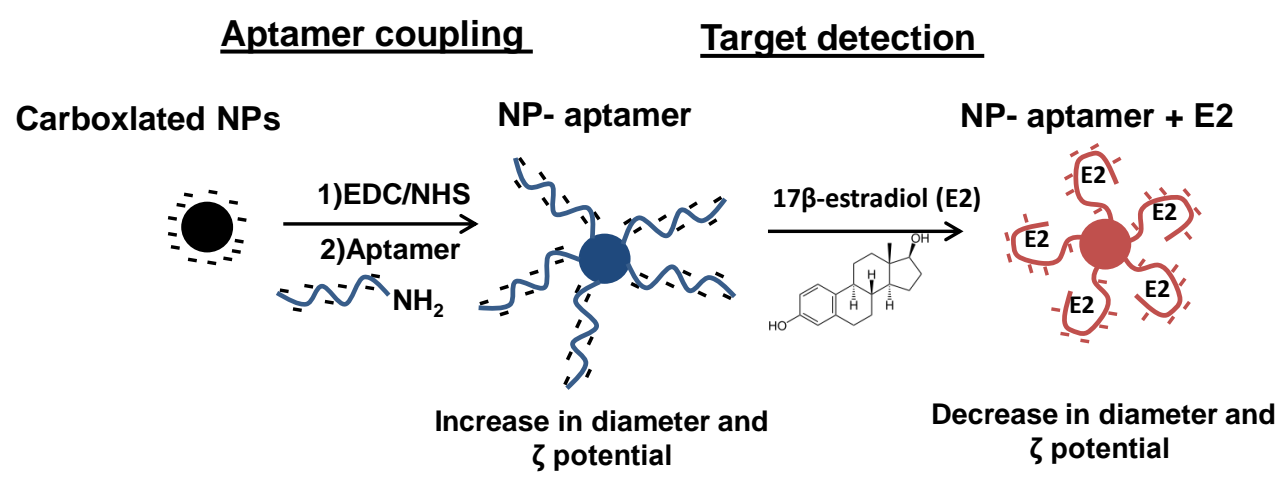

Scheme 2.1: Schematic overview of the size based aptasensor developed in this study.

The size based aptasensor was demonstrated with a previously isolated 75-mer aptamer to target the hormone E2, as well as with a known aptamer for adenosine for proof of generality. The proposed size based aptasensor is able to detect the presence of $\sim 5 \mathrm{nM}$ E2 in buffered solution by measuring changes in particle sizes via both DLS and TRPS - a recently developed particle-by-particle sizing technique. ${ }^{75195}$ Additionally, a clear correlation between NP-aptamer size contraction and surface $\zeta$-potential was observed, due to the redistribution of charge when the polyanionic aptamer folds around its target, thus extending the range of simple means to transduce binding signals in this system.

\subsection{Overview of TRPS and light scattering techniques}

\section{NP size by TRPS}

TRPS is an extension of the resistive pulse sensing technique that detects individual particles traversing a single pore when they physically disrupt the ionic current flowing through the pore, between two sides of a fluid cell. ${ }^{95}$-97 Generally, resistive pulse sensors measure the increased electrical resistance caused by a particle passing through a pore filled with electrolyte. ${ }^{98}$ For a cylindrical pore (the simplest case), the relative change in resistance $\Delta R / R$ is described by Eq. 2.1. 99

$$
\frac{\Delta R}{R}=\frac{d^{3}}{D^{2} L}
$$

where $\mathrm{D}$ is the pore diameter, $\mathrm{L}$ the pore length, and $\mathrm{d}$ the particle diameter.

TRPS, (the commercial instrument is known as the qNano) shown in Fig. 2.1, is one of the latest advancement in this field and relies on the use of a mechani- 
cally active thermoplastic polyurethane membrane containing a single conically shaped pore (Fig. 2.1p). The pore is made by a mechanical puncturing using a tungsten needle that produces large (in the range of $\mu \mathrm{m}$ ) and small (in the range

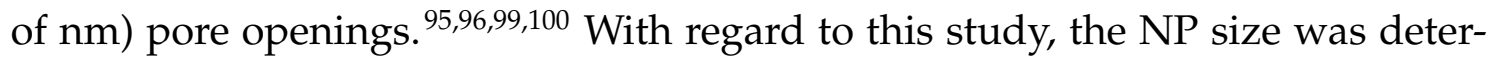
mined based on calibration with standard particles of a known size. Upon placing the NP sample in the upper fluid cell (while the lower fluid cell contains the same electrolyte used to suspend the particles), applying external voltage, and tuning the macroscopic stretch of the membrane, individual particles start migrating through the pore (Fig. 2.1p). Individual particles disrupt the ionic current by creating events seen as inverted peaks (Fig. 2.1k) characterized by magnitude $(\Delta \mathrm{I})$ and full width half maximum (FWHM) duration that correspond to the size and speed of the particles, respectively. Scatter plots and histograms of particle size can be obtained after calibration (Fig. 2.1 d and Fig. 2.17).

In addition to size measurement, TRPS can provide valuable information from the speed of the particles when they traverse the pore construction. ${ }^{101}$ Therefore, understanding the factors that affect the particle transport will further assist the utility of this technique as a sensor. There are various mechanisms that govern particle flux $(\mathrm{J})$ through the pore construction, described by Nernst-Planck equation (Eq. 2.2),

$$
J=J_{e o}+J_{e p}+J_{d i f f}+J_{p d}
$$

where $\mathrm{J}_{\mathrm{eo}}$ is electro-osmosis, $\mathrm{J}_{\mathrm{ep}}$ is electrophoresis, $\mathrm{J}_{\text {diff }}$ is diffusion, and $\mathrm{J}_{\mathrm{pd}}$ is pressure-driven transport. ${ }^{97}$ Recent work by Vogel et al. ${ }^{100}$ revealed that electrophoresis and electro-osmosis (the pore has a surface potential of approximately $-8 \mathrm{mV}$ in the standard electrolyte $100 \mathrm{mM} \mathrm{KCl}$ ) and inherent pressure (46 Pa per $40 \mu \mathrm{L}$ ) are the main transport mechanisms when no external pressure is used. 

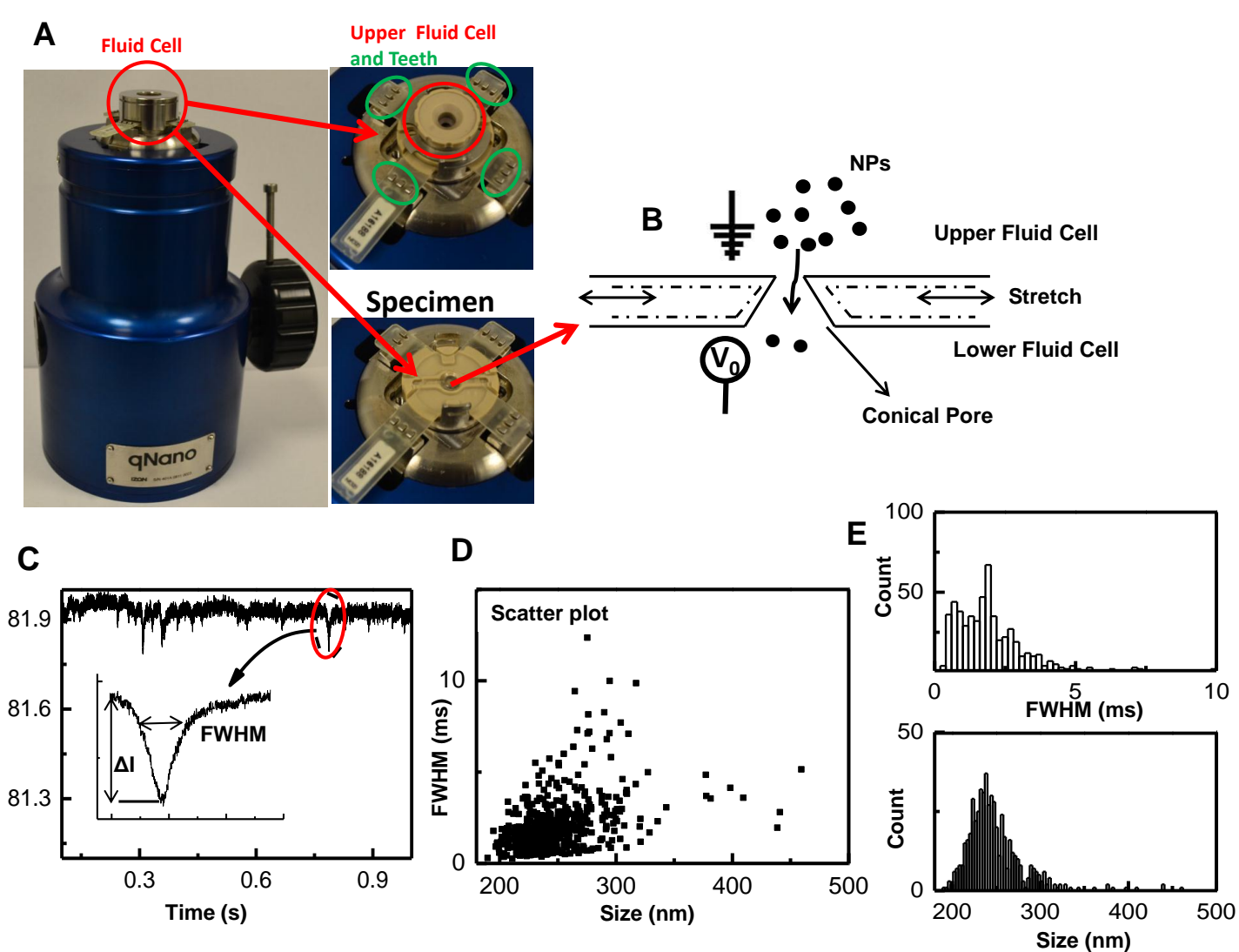

Figure 2.1: a) The qNano system includes fluid cell. The elastic pore membrane is mounted between two halves of the fluid cell, each of which contains an electrode $(\mathrm{Ag} / \mathrm{AgCl})$, and the membrane is attached to adjustable stretching teeth, actuated by rotating the black handle on the side of the blue body. b) Schematic cross-section of the stretchable elastic pore showing particles traversing the conical pore. c) Background trace containing blockade events generated by the particles. Measurement of event magnitude $(\Delta \mathrm{I})$ and FWHM duration are used to characterise particle size and speed respectively. d) Scatter plot of 500 blockade events characterised by size (nm) and FWHM duration (ms). e) Histogram distributions of size and FWHM duration.

\section{NP size by light scattering technique}

DLS, also known as photon correlation spectroscopy, is a technique used for measuring particle size and size distribution in suspensions. This technique is based on the random thermal motion, Brownian motion, of spherical particles which causes scattering of the incident laser light. The diffusion constant of the particles is measured and the size of the particles is calculated according to the StokesEinstein relation, Eq. 2.3, 102 


$$
D_{h}=\frac{k_{B} T}{3 \pi \eta D_{t}}
$$

where $\mathrm{D}_{h}$ is the hydrodynamic size, $\mathrm{D}_{t}$ the diffusion coefficient (the primary parameter obtained from DLS measurements), $\mathrm{k}_{\mathrm{B}}$ the Boltzmann constant, $\mathrm{T}$ the temperature, and $\eta$ the solvent viscosity.

The scattered light is collected at a given angle (forward scattering $90^{\circ}$ or back scattering $173^{\circ}$ ), the fluctuation is recorded over time, and the decay time of the signal autocorrelation is used to calculate the $\mathrm{D}_{t}$ of the particles. Smaller particles will result in a faster decay due to their faster movement, unlike larger particles. It should be noted that the size measured by this method is the hydrodynamic size, which is the actual size of a particle in addition to the attached ions or molecules. 103

\section{$\zeta$-potential potential by light scattering technique}

The presence of net charge density on the surface of a particle in solution gives rise to the existence of an electric double layer. The electric double layer describes the increased concentration of counter ions at the interfacial region of the particle. The strongly bound ions form the first layer around the particle called the Stern layer and the less firmly bound ions form the second layer called the Slipping plane, as shown in Fig. 2.2. $\zeta$-potential is measured at the surface of the slipping plane (Gouy layer) and reflects the potential difference from the bulk solution. The magnitude of the $\zeta$-potential is highly influenced by the $\mathrm{pH}$ and ionic strength of the medium and is a meaningless parameter without the knowledge of these values. Additionally, $\zeta$-potential provides valuable information regarding colloidal stability, i.e. nanoparticles with $\zeta$-potential values of -30 or $+30 \mathrm{mV}$ are considered to be stable. $104 \mid 105$ 


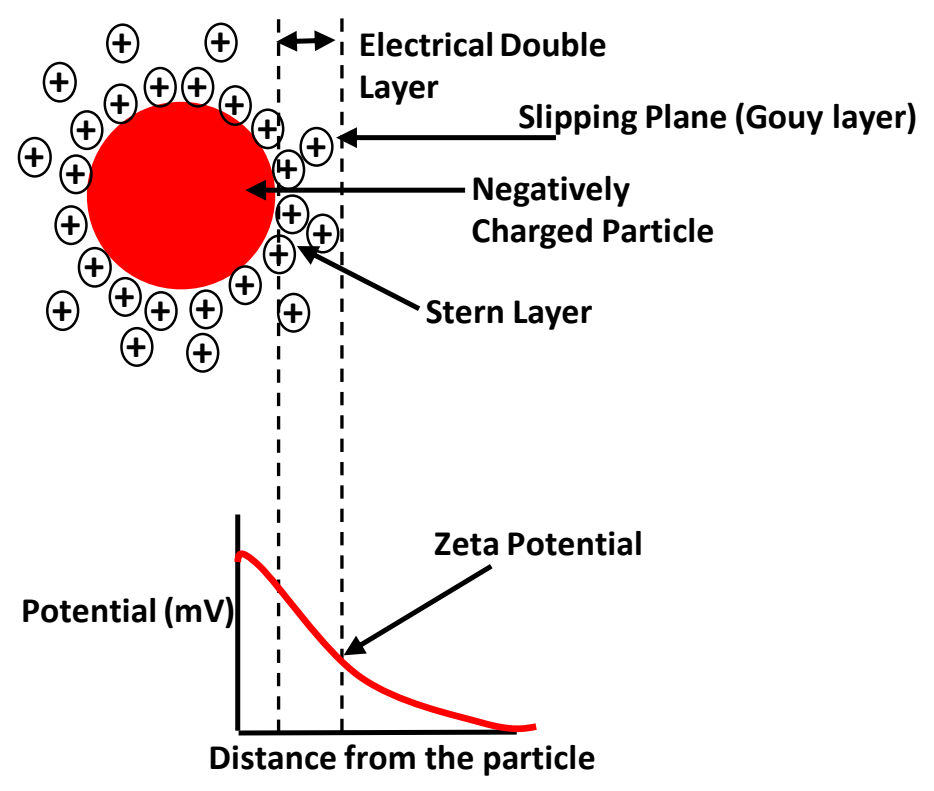

Figure 2.2: Schematic representation of a particle and associated electrical double layer. The particle is negatively charged, hence the layer of tightly associated positive ions present in the Stern layer. Inset is a graph describing the layers surrounding a negatively charged particle, including the $\zeta$-potential at the Slipping plane.

$\zeta$-potential can be obtained using the light scattering method (phase analysis light scattering, PALS) from the calculation of electrophoretic mobility of charged particles in a medium with known dielectric constant and viscosity. The colloidal sample containing nanoparticles is placed in a capillary cell and an external voltage is applied across the cell using gold electrodes. The particles will move towards the oppositely charged electrode. After reaching equilibration with the surrounding medium (acceleration and temperature), the sample is illuminated with laser light which is shifted to a greater frequency due the movement of the particles. By combining with a reference beam, the intensity fluctuation of the scattered light is generated and the shift in phase is monitored (PALS). Fluctuation frequency of the scattered light is proportional to the electrophoretic mobility $\left(\mathrm{U}_{E}\right)$ of the traveling particles. After correlation, $\mathrm{U}_{E}$ is calculated. Finally, $\zeta$-potential is calculated using Henry's equation Eq. 2.4.

$$
U_{E}=\frac{2 \epsilon \zeta-\text { potential } f\left(k_{a}\right)}{3 \eta}
$$

under known experimental conditions including electrolyte viscosity $(\eta)$, dielectric constant $(\epsilon)$, and Henry's function $f\left(\mathrm{k}_{\mathrm{a}}\right) .106$ 


\section{DLS versus TRPS}

DLS is considered to be a bulk analysis technique. Particle size can be obtained in most solvents and over a wide size range (from a few nanometers up to several microns). ${ }^{99}$ DLS does not provide detailed analysis of multi-mode systems (i.e. a population with two distinct sizes or more). 107 Presence of small amount of aggregates might affect accurate sizing of a given population. ${ }^{99}$

On the other hand, TRPS is a particle-per-particle characterising technique, based on a number distribution. TRPS was reported to discriminate between mixed nanoparticle samples $(220,330$, and $410 \mathrm{~nm}) .[107$ The pore diameter can be changed, due to the ability of actuating the elastic membrane, to remove temporary blockages as well as enhancing measurement sensitivity and resolution. However, TRPS measurements are limited to conducting electrolyte and relatively large particle sizes. $\frac{.9107}{}$

\subsection{Results and discussion}

\subsubsection{Aptamer selection and characterisation}

The aptamer selection and dot blot characterisation were done by Dr. Shalen Kumar and Professor Kenneth P. McNatty, reproductive biology lab, School of Biological Sciences at VUW. The E2 aptamer was isolated from a library of synthetically generated and random ssDNA containing $7.2 \times 10^{14}$ molecules by employing a typical process of SELEX. ${ }^{108}$ After selection round 18, the 75-mer sequence, outlined in Fig. 2.3, was selected as one of the four most promising and sensitive aptamers, and was able to detect E2 as low as $37.5 \mathrm{nmol}$ and also, up to $300 \mathrm{nmol}$, using a modified dot blotting assay as shown in Fig. 2.5. 


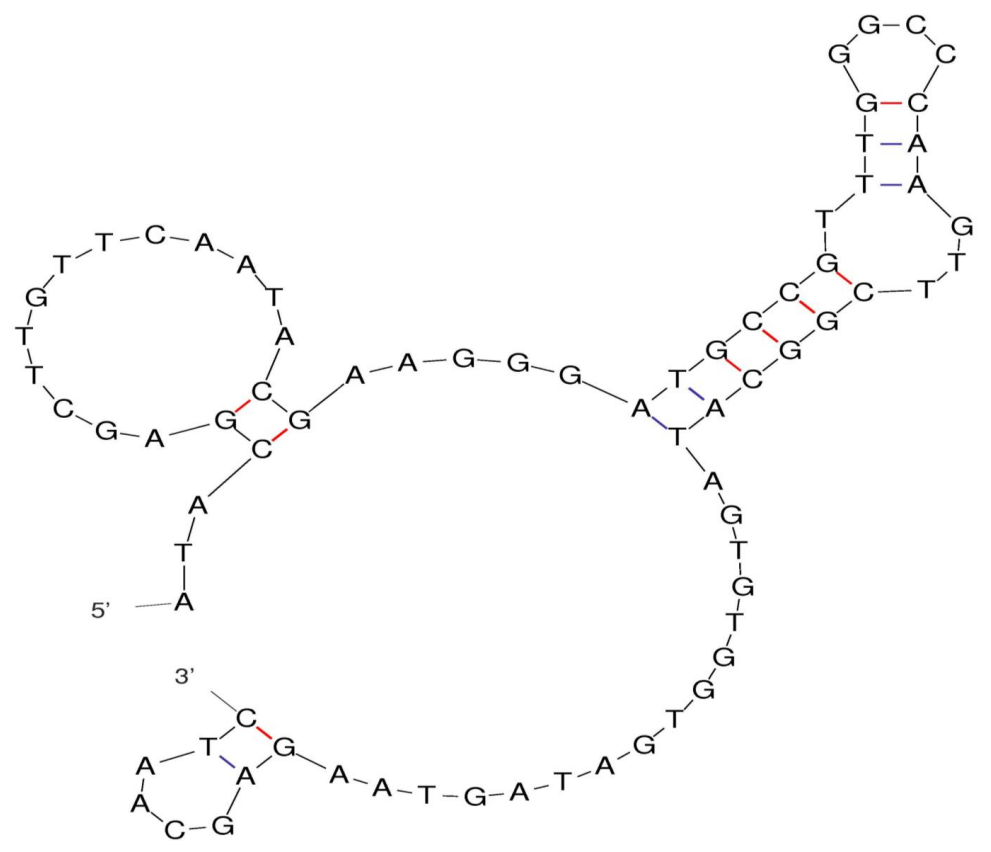

Figure 2.3: Secondary structure of the 75-mer aptamer. The secondary structure was predicted by an online web server Mfold ${ }^{109}$ under the following conditions: $25^{\circ} \mathrm{C}, 10 \mathrm{mM} \mathrm{NaCl}$ and $0.1 \mathrm{MgCl}_{2}$ (BWB conditions).

\section{$K_{\mathrm{D}}$ measurements}

In order to determine the $K_{\mathrm{D}}$ value of an aptamer, a constant concentration of the aptamer is titrated with an increasing concentration of the target, then the bound or unbound fraction of the target is determined. $\frac{110}{10}$ Bound and unbound fractions were previously separated for aptamers interacting with small molecules including bisphenol $\mathrm{A}^{1111}$ via a method known as equilibrium filtration. An aptamertarget complex can be separated from the free target using nitrocellulose (NC) filters with a specific molecular cut-off size. This method was initially examined to obtain the $K_{\mathrm{D}}$ of the 75-mer aptamer. However, E2 was found to non-specifically adsorb on the filters. This was indicated from filtering serial concentrations of E2 through the NC membranes and comparing the fluorescence signals with a calibration curve, Fig. $2.4 \mathrm{~b}$. As a result, it was concluded that equilibrium filtration is not suitable for the $K_{\mathrm{D}}$ measurement of the 75-mer aptamer. 
A

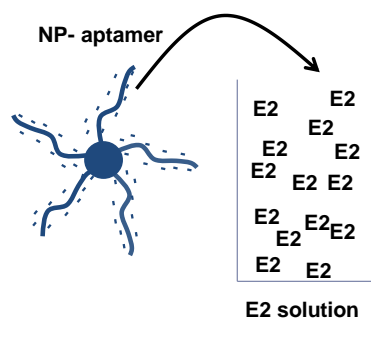

B

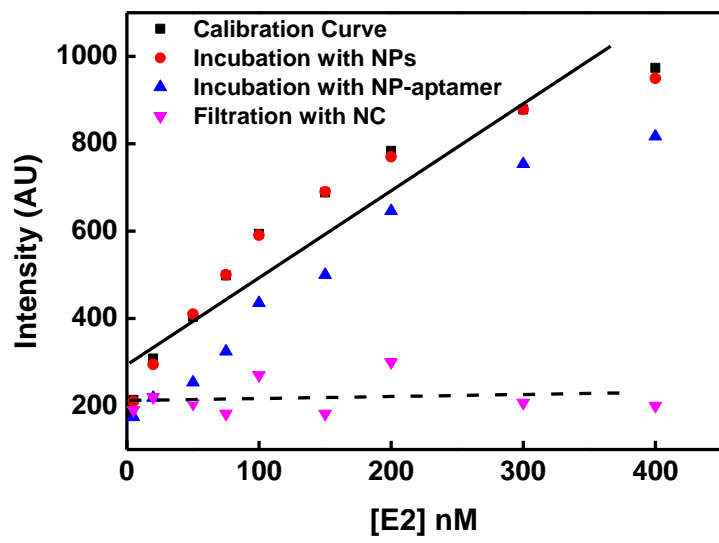

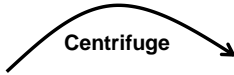

NP- aptamer + E2
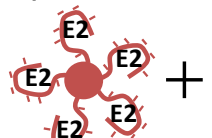

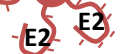

Bound E2 Supernatant sample

Figure 2.4: a) Schematic illustration of isolating the bound and unbound fractions of E2 using the 75-mer aptamer coupled to carboxylated polystyrene NPs. b) Shows a standard calibration curve of E2 fluorescence at $310 \mathrm{~nm}$, control experiments of incubating E2 concentrations with the bare NPs to examine the nonspecific binding, incubation with NP-aptamer conjugate for $K_{\mathrm{D}}$ determination and filtration E2 concentrations through the nitrocellulose (NC) filters.

Alternatively, the 75-mer aptamer $K_{\mathrm{D}}$ was determined via coupling the aptamer to carboxylated polystyrene NPs (following Scheme 2.2 that will be discussed later) then incubating the NP-aptamer conjugate with serial concentrations of E2 and determining the unbound fraction of E2 via centrifuge separation of NPaptamer + E2 complex followed by fluorescence measurements of the unbound E2, as shown in Fig. 2.4a. There was no nonspecific interaction between E2 molecules and the bare NPs indicated from the approximately identical signals of the calibration curve of E2 and the concentrations of E2 after incubation with the bare NPs (Fig. 2.4 b). The concentration of unbound E2 was determined using a calibration curve of E2. The fluorescence signals of E2 for the calibration curve reached the limit of linearity at higher concentrations (300 nM and 400 nM, Fig. 2.4 , black symbols) due to an unknown interference or an instrumental defect. The data points were fitted to a straight line to determine the unbound fraction of E2 after separation following the scheme depicted in Fig. 2.4a. A polynomial fitting (a curve) could also be used to predict the unbound fraction of E2 as well. As shown in Fig. 2.5p, a $K_{D}$ of $25 \pm 2.5 \mathrm{nM}$ (standard deviation of the fit) was obtained by plotting the normalised 1 /unbound fraction $\left(f_{a}\right)$ against $1 /$ [E2] of the original E2 concentration and applying Eq. 2.5. 110 


$$
\frac{1}{f_{a}}=\frac{K_{D}}{[E 2]}+1
$$

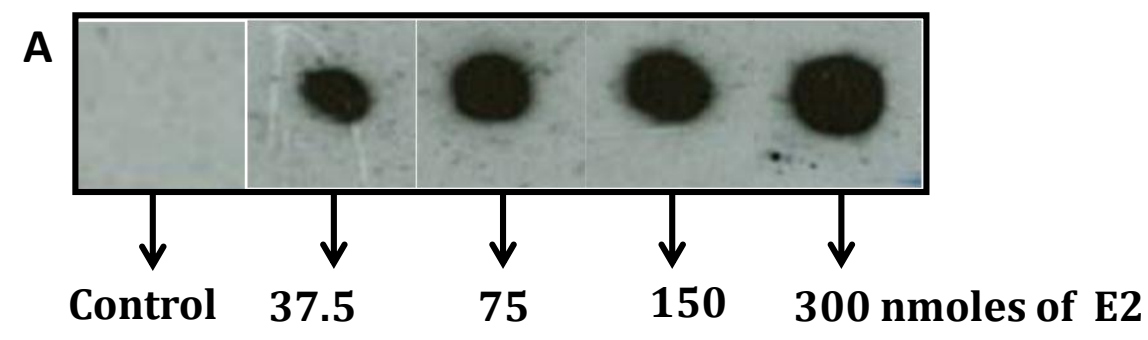

B

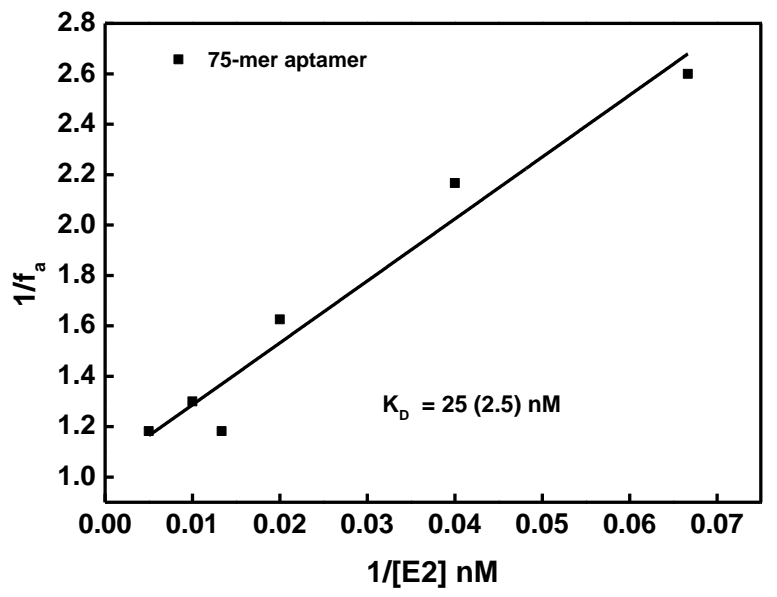

C

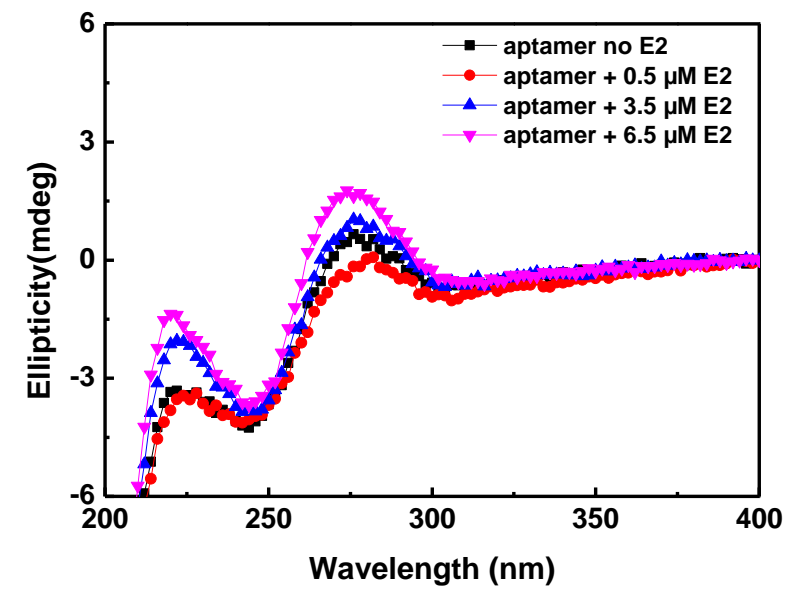

Figure 2.5: a) Dot blot detection of serial amounts of immobilised E2 on nitrocellulose and chemoluminescence visualisation of the bound E2 aptamer. b) Determination of the $K_{D}$ of the 75-mer aptamer from a plot of $1 /$ [E2] against $1 / f_{a}$ (number in brackets indicate standard deviation of the fit). c) CD spectra of $400 \mathrm{nM} 75$-mer aptamer and the independent detection of $0.5,3.5$ and $6.5 \mu \mathrm{M}$ concentrations of E2.

\section{Circular dichroism characterisation}

The optical bands of nucleotides exhibit a property in circular dichroism (CD) due to the lack of a center of symmetry (i.e. presence of chirality). CD signals 
result from unequal absorption of left-handed and right-handed polarised light interacting with a molecule. CD bands in the far UV region (200-290 nm) arise from the abundance of amide bonds in nucleotides. ${ }^{112}$ A homogeneous solution detection of E2 could be performed using CD spectroscopy ${ }^{73}$ as shown in Fig. 2.5. E2 aptamer is classified as a B-form oligonucleotide which produces a CD spectrum characterized by positive maximum and negative minimum ellipticities around $280 \mathrm{~nm}$ and $248 \mathrm{~nm}$ respectively. ${ }^{113[114}$ With increasing concentrations of E2, an increase in the positive ellipticity maximum at $280 \mathrm{~nm}$ was observed, consistent with the binding-induced transition to a folded conformation featuring more stem-loop structures. ${ }^{115}$ Nevertheless, the relatively high concentrations of aptamer $(400 \mathrm{nM})$ required to measure the CD spectra means that binding is only observed when the aptamer is saturated with relatively high (micromolar) concentrations of E2. Having demonstrated that the aptamer has a strong affinity for E2 and undergoes a binding-induced conformational switch, the aptamer was applied in the sensor illustrated in Scheme 2.1.

\subsubsection{Generation and characterization of NP-aptamer}

Carboxylated polystyrene NPs were selected for functionalization with aptamers owing to: i) the availability of highly monodisperse samples (size distribution full width half maximum (FWHM) of $\sim 50 \mathrm{~nm}$ and polydispersity index (PDI) of 0.025 according to TRPS and DLS respectively) of a size that is large enough to be measured reliably, yet small enough that the aptamer surface coating can have a large relative effect on the properties of the particle; ii) the ability to apply amide coupling chemistry to covalently attach amine terminated aptamers to the carboxylate surface; and iii) their stability in aqueous sensing environments with moderate ionic strength.

\section{Activation of NPs and aptamer coupling}

As shown in Scheme 2.1, carboxylated polysterene NPs with a nominal diameter of $217 \mathrm{~nm}$ were activated towards coupling in water with $0.1 \mathrm{M} 2$-(N-morpholino) ethanesulfonic acid (MES) buffer through the addition of N-(3-dimethylaminopropyl) N-ethylcarbodiimide (EDC) and N hydroxy-succinimide (NHS) to form an NHS ester coating on the NP surface. The esters subsequently react with the amine terminated aptamers to form stable amide linkages tethering the aptamers to the NP, as shown in Scheme 2.2 . 

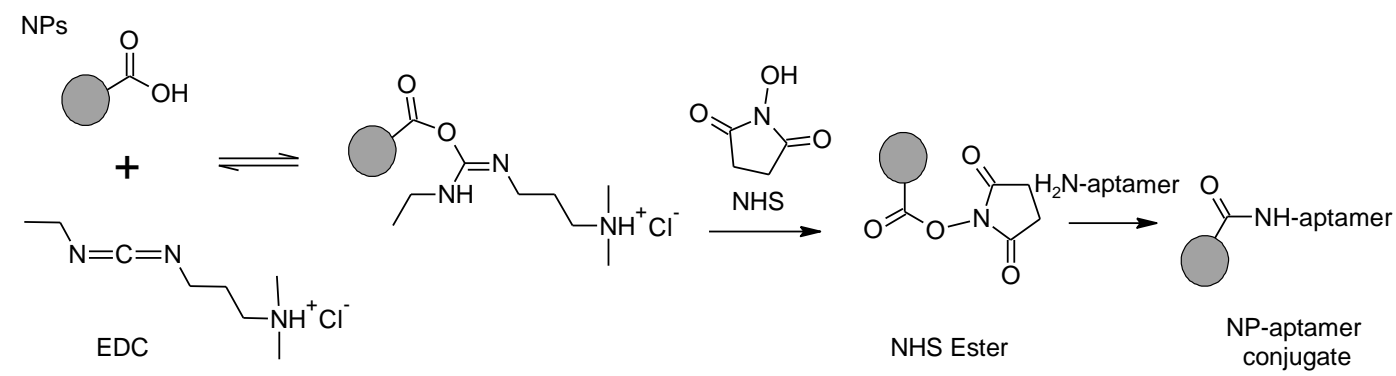

Scheme 2.2: EDC/NHS coupling chemistry.

The success of functionalization could be determined by measuring the $\zeta$-potential of NPs after isolation via centrifugation and redispersion in MES buffer. Table 2.1 shows that the formation of the (neutral) NHS ester was confirmed via a increased in $\zeta$-potential value to $-32 \mathrm{mV}$ compared with $-36 \mathrm{mV}$ for the bare NPs which have an anionic carboxylate surface. The $\zeta$-potential of the NPs then decreased to -41.3 $\mathrm{mV}$ upon coupling the polyanionic aptamer. It was verified that these observations reflect a covalent functionalization rather than non-specific adherence to the NP surfaces because the bare NP $\zeta$-potential was obtained when the coupling agents were omitted.

Table 2.1: Absolute values of $\zeta$-potential obtained via phase analysis light scattering for populations generated during the coupling of aptamers to NPs and during the detection of E2.

\begin{tabular}{lll}
\hline & \multicolumn{2}{c}{ Zeta Potential /mV $(\mathrm{STD}, \mathrm{n}=3)$} \\
\cline { 2 - 3 } Samples & in MES buffer & in BWB buffer \\
\hline NPs & $-36.0(0.1)$ & $-32.5(1.0)$ \\
NP-NHS & $-32.3(2.0)$ & Not measured \\
NPs + aptamer no EDC/NHS & $-36.0(0.8)$ & Not measured \\
NP-aptamer & $-41.3(0.6)$ & $-33.5(0.5)$ \\
NPs + 50 nM E2 & Not measured & $-32.0(1.0)$ \\
NP- aptamer + 50 nM E2 & Not measured & $-28.2(0.5)$ \\
\hline
\end{tabular}

\section{Aptamer surface coverage}

The NPs used in this study have carboxyl groups on their surface: $5 \mathrm{nmol}$ of carboxylates for every $1.04 \times 10^{10}$ particles as determined by the manufacturer. Several amounts of NPs $\left(1.04 \times 10^{10}, 2.08 \times 10^{10}, 3.12 \times 10^{10}\right.$ and $\left.4.16 \times 10^{10}\right)$ were exposed to a fixed amount $(0.1 \mathrm{nmol})$ of a fluorescently labeled 76-mer aptamer (F-aptamer), then the NP-F-aptamer conjugates were separated from the mix- 
ture when the coupling was finished as described in the Experimental Section. It should be noted that the F-aptamer was previously used by Kim et al ${ }^{7}$ for the detection of E2 and the sequence is different from the sequence reported here. Fluorescence was measured in both NP-F-aptamer and the supernatant samples.

As shown in Fig. 2.6, the fluorescent measurements result from a covalent functionalization rather than non-specific adherence to the NP surfaces because the exposure of NPs $\left(2.08 \times 10^{10}\right)$ to F-aptamer without activating agents (EDC/NHS) resulted in no interaction (circled data points). This observation is consistent with the previously noted results regarding $\zeta$-potential. Further, it was observed that the $0.1 \mathrm{nmol}$ was completely bound by the second amount of NPs $\left(2.08 \times 10^{10}\right)$ and the remaining amounts of NPs gave approximately the same fluorescent signal as shown in Fig. 2.6. This observation shows that the lower NPs concentrations have a sufficiently high F-aptamer:NP ratio to saturate the NP surfaces, whereas the aptamers are coupled at lower density as the NP concentration is increased.

Then, the fluorescent intensities of the samples with $1.04 \times 10^{10}$ and $2.08 \times 10^{10}$ particles were converted to concentrations and eventually number of nmol using a standard calibration curve constructed of several concentrations of F-aptamer mixed with $2.08 \times 10^{10} \mathrm{NPs}$. It was found that the number of F-aptamers per particle is $2.66 \times 10^{3}, 2.60 \times 10^{3}, 1.70 \times 10^{3}$ and $2.66 \times 10^{3}$ corresponding to the measurements in the samples containing $1.04 \times 10^{10}$ and $2.08 \times 10^{10}$ particles and their supernatants respectively. The overall average F-aptamer density is $2.40 \mathrm{x}$ $10^{3}$ per particle (with a standard deviation of $0.4 \times 10^{3}, \mathrm{n}=4$ ) giving $25 \mathrm{~nm}^{2}$ free space for each aptamer. It should be noted that there is an additional uncertainty of approximately $10 \%$ from the NPs lost during the centrifuging process. 


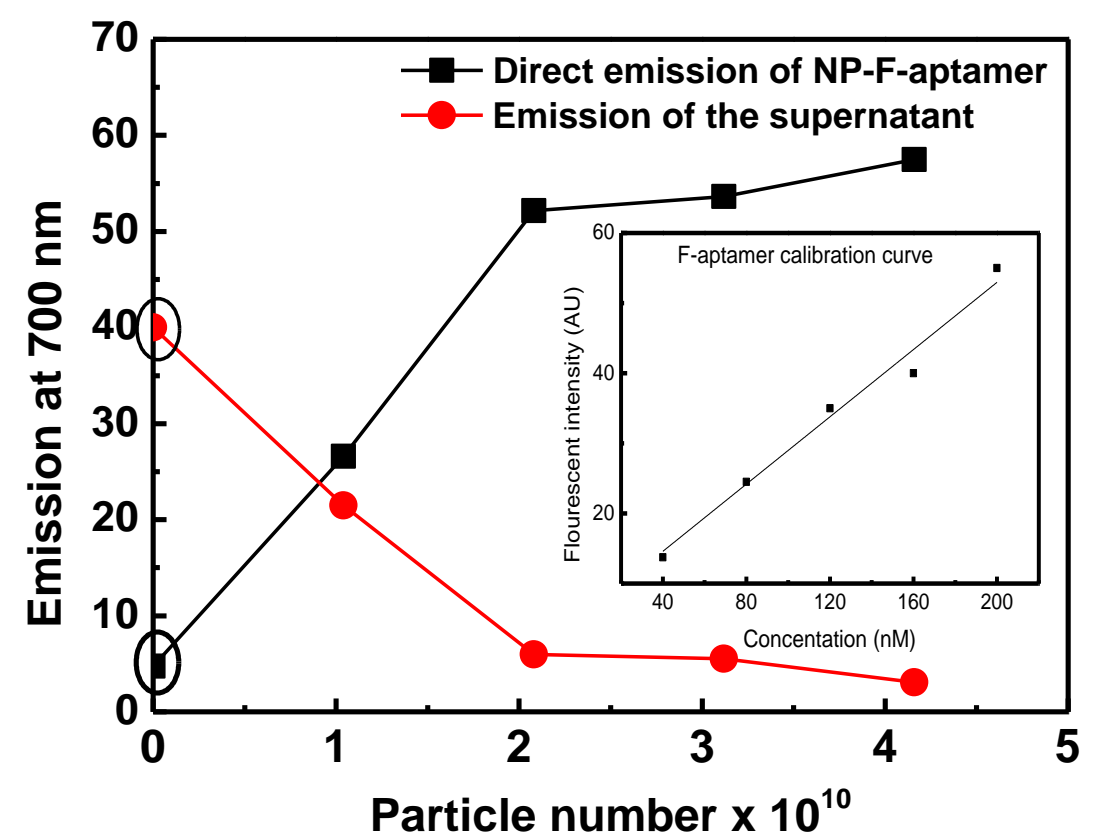

Figure 2.6: Fluorescence measurements of samples resulting from the reaction of several independent NP numbers $\left(1.04 \times 10^{10}, 2.08 \times 10^{10}, 3.12 \times 10^{10}\right.$ and $\left.4.16 \times 10^{10}\right)$ with a fixed amount $(0.1 \mathrm{nmol})$ of fluorescently tagged aptamer $(\mathrm{F}-$ aptamer). Circled data points represent the results of a control sample: exposure of $2.08 \times 10^{10} \mathrm{NPs}$ to $0.1 \mathrm{nmol}$ F-aptamer with no presence of the activating agents (EDC/NHS). Figure-inset is a calibration curve constructed of serial concentrations of the F-aptamer mixed with NPs $\left(2.08 \times 10^{10}\right.$ particles $)$. Lines joining data points are drawn to guide the eye.

\section{Size and duration (FWHM) based characterisation of aptamer coupling}

The first step towards using NP size measurement techniques for E2 sensing is to confirm that aptamer coupling results in a clearly measurable increase in NP size. As shown in Fig. 2.8 a , DLS resolves an increase in average hydrodynamic particle diameter from $250 \mathrm{~nm}$ to $300 \mathrm{~nm}$ upon functionalization with E2 aptamer (using the average values of the distribution and from $\sim 220 \mathrm{~nm}$ to $250 \mathrm{~nm}$ using the mode values). In addition to DLS, the TRPS technique was used to resolve particle sizes. Fig. 2.8 shows that TRPS resolves an approximately $20 \mathrm{~nm}$ increase in the mode diameter of NPs as a result of coupling the E2 aptamer. It is apparent that, although DLS and TRPS measurements reveal the same trend, the absolute diameters differ. This is not unexpected, because DLS gives an average based on a number distribution which is derived from the intensity distribution of scattered light, whereas TRPS number distributions are based upon particle-by-particle measurements. It is well recognised that DLS is a bulk analysis method and does not provide detailed information of the particle number 
distribution, as recently explored in comparison with TRPS. $107 / 116$ As a result, any possible presence of aggregates, observed in this study as shown in Fig. 2.8a, might affect absolute sizing. Additionally, DLS provides the hydrodynamic size, measured from the random thermodynamic motion of the particles (Brownian motion), which is greater than the actual size of the particle. ${ }^{117}$ The other possible reason could the comparison between the mode data from TRPS and average data from DLS. The maximum theoretical increase in NP diameter can be estimated as approximately $50 \mathrm{~nm}$ by considering the fully extended length of the 75-mer E2 aptamer, assuming $0.34 \mathrm{~nm}$ spacing between the backbone bases. ${ }^{114}[118$ A more relaxed aptamer conformation (or distribution of conformations) will result in somewhat less extension, in line with what is observed. It should be noted that there was no obvious change in the size of the particles under the scanning electron microscope (SEM) imaging (refer to Fig. 2.7), presumably due to the lack of contrast, limited resolution, and the inability to carry out SEM in the preferred environment of buffered electrolyte solution.
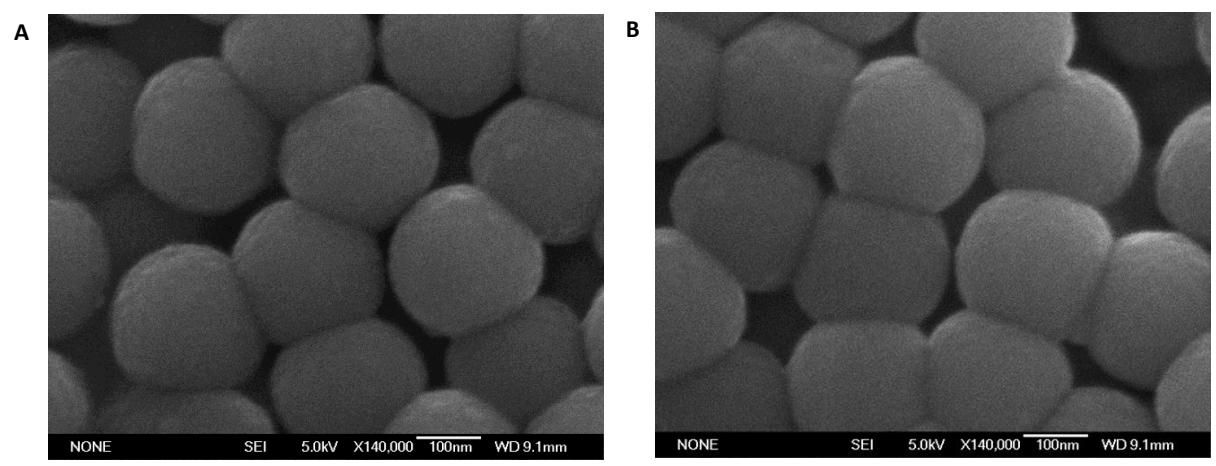

Figure 2.7: SEM characterisation of coupling the aptamer to $217 \mathrm{~nm}$ NPs a) before coupling and b) after coupling.

It was also found that the presence of aptamers increases the duration (FWHM) of resistive pulses compared with that of the bare NPs, as shown in Fig. 2.8p. In spite of the expectation that increased surface potential will increase the electrophoretic mobility of NPs, the observed reduction in NP-aptamer speed can be attributed, in the main, to the steric drag imparted by the aptamers on NPs when passing through the narrow pore. The same effect was observed when $\lambda$-DNA was coupled to silica NPs and measured via TRPS. 119 

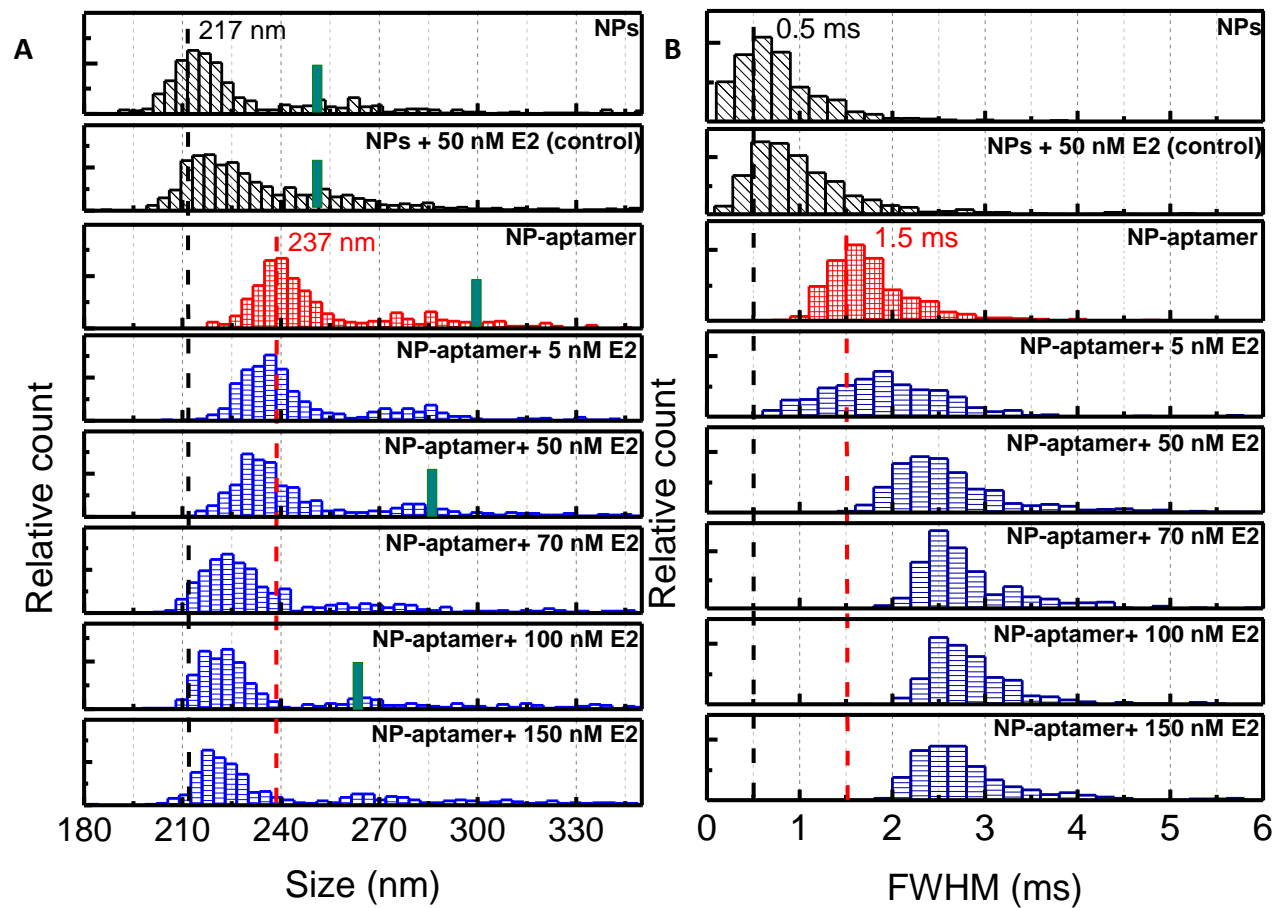

Figure 2.8: a) Size histograms of different samples obtained by TRPS during the detection process of E2. The locations of green bins indicate the averages of size distributions by number obtained from DLS. (b) FWHM histograms of different samples obtained by TRPS during the detection process of E2. The dashed vertical lines are aligned to the modal bars of the bare NPs (black) and NP-aptamer (red). The bin sizes, which are a reasonable indicator of the uncertainty, are 3.5 $\mathrm{nm}$ and $0.2 \mathrm{~ms}$ for size and duration (FWHM) respectively.

\subsubsection{Detection of E2}

Having established that TRPS and DLS can resolve the effect of coupling aptamers to NPs, the size based scheme was applied to the detection of the small molecular target. Sensing was carried out in the binding washing buffer (BWB) used for aptamer generation. Samples were prepared to ensure that their compositions differed only by E2 concentration in the $\mathrm{nM}$ range. Fig. 2.8 a shows that increasing concentrations of E2 results in progressively smaller NP-aptamer diameters, whether measured via TRPS or DLS. At an E2 concentration of $\sim 100$ $\mathrm{nM}$, where binding appeared to be saturated, the measured NP-aptamer diameter $(220 \mathrm{~nm})$ approached that of the bare NP population $(217 \mathrm{~nm})$, indicating that the E2 bound aptamers were adopting a substantially more compact conformation than for the NP-aptamer conjugates $(237 \mathrm{~nm})$. The concentration of the aptamer in the detection solutions was $225 \mathrm{nM}(0.022 \mathrm{nmol})$ and the highest detectable concentration of E2 was $150 \mathrm{nM}(0.015 \mathrm{nmol})$. Based on the measured $\mathrm{K}_{\mathrm{D}}$ of $25 \mathrm{nM}$, it was expected $85 \%$ of the aptamers to be bound with $150 \mathrm{nM}$ E2, and 
$16 \%$ for the lowest concentration of E2 that was detected ( $5 \mathrm{nM}$ ). A control sample omitting the reagents required for coupling the aptamer to the NPs confirmed that the observed sensitivity arose from the specific response of the NP-aptamer conjugates binding to E2 as shown in Fig. 2.8a (second panel from top).

Fig. 2.8p shows the distributions of pulse duration (FWHM) measured for the same set of samples in TRPS. The pulse duration of NP-aptamers was found to increase from $1.5 \mathrm{~ms}$ to $2.7 \mathrm{~ms}$ as the concentration of E2 increased, again saturating at approximately $100 \mathrm{nM}$. On the one hand, it might be expected that the size contraction observed to result from E2 binding would reduce the steric drag effects encountered by the unbound NP-aptamer, which would result in shorter pulse durations. On the other hand, the $\zeta$-potential measurements summarized in Table 2.1 showed that E2 binding resulted in a less surface potential of NPaptamers, presumably coupled to the conformational switch of the polyanionic aptamer. Reduced surface potential will likely reduce the electrophoretic mobility of the NP-aptamer conjugates bound to E2, which in turn opposes the effect of reduced steric drag and may explain the observed increase in pulse duration.

\section{Linearity and specificity}

Fig. 2.9a provides evidence that the size based method of E2 detection is both robust and reproducible: for three independent experiments starting from the functionalization of carboxylated NPs, the variation was less than $20 \%$ for NPaptamer size and the pulse duration measurements (FWHM). Fig. 2.9a illustrates that a strong correlation exists between NP-aptamer size and the pulse duration measurements, confirming that both have a common origin. The method is able to detect E2 reliably at concentrations as low as $5 \mathrm{nM}$, which places the assay limit of detection in the concentration range for the human response to ingested E2. 120 


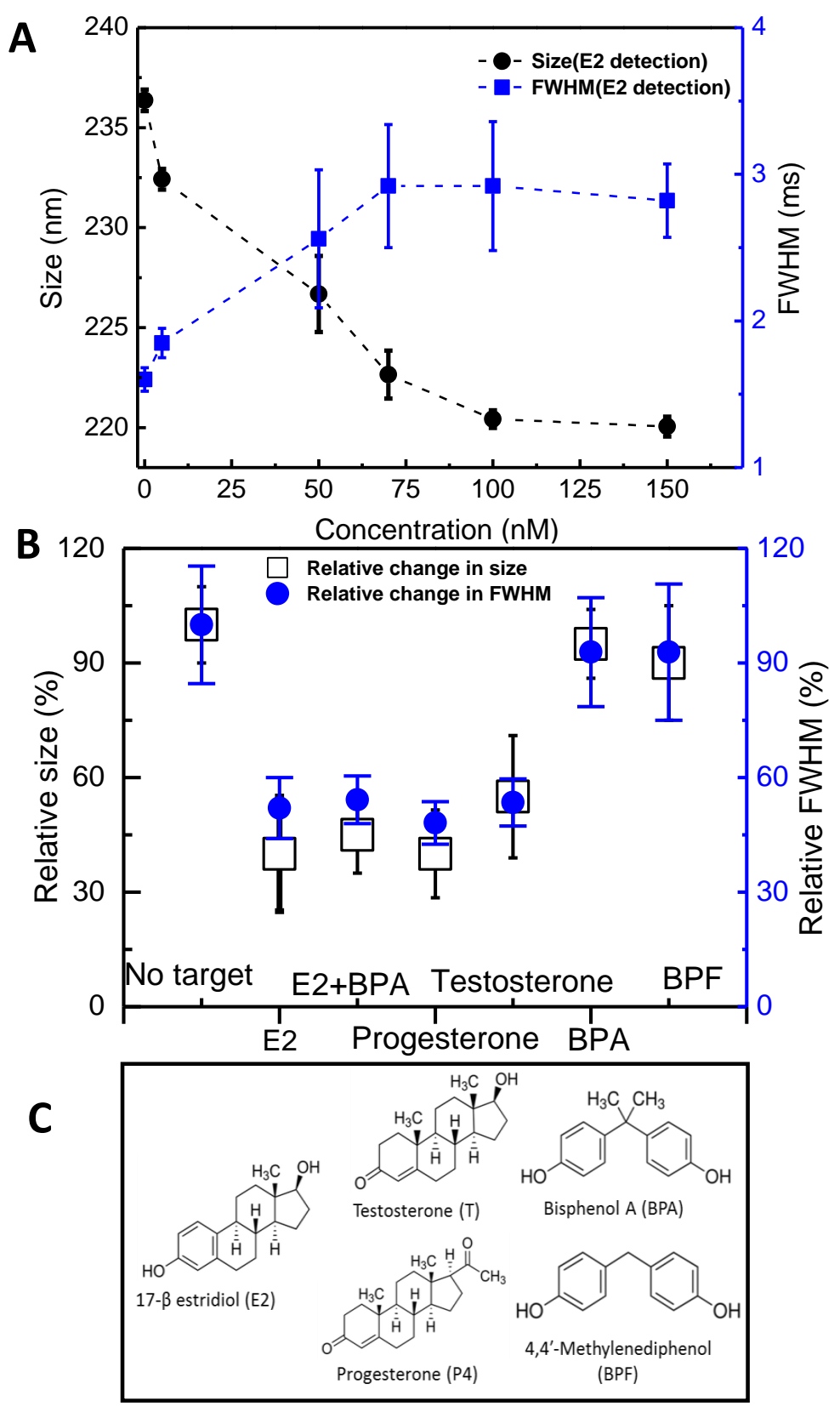

Figure 2.9: a) Mode values of size and FWHM obtained by TRPS during the detection of E2 (error bars indicate the standard deviation of the average from three independent experiments). b) Relative size and FWHM obtained by TRPS during the specificity examinations of $50 \mathrm{nM}$ of E2, E2 + BPA, progesterone, testosterone, BPA and BPF (error bars indicate the standard deviation of the average from three independent measurements). Relative change (\%) in size was calculated from the $20 \mathrm{~nm}$ extension of aptamer added to NPs and the relative change (\%) in FWHM duration was calculated from the duration of NP-aptamer (1.5 ms). c) Chemical structures of the molecules tested in this study.

The method is able to detect E2 in the range of 5 to $150 \mathrm{nM}$ with an acceptable linearity ( $\mathrm{R}^{2}$ of 0.97 and 0.89 for size and FWHM respectively) over the range from $5 \mathrm{nM}$ to $75 \mathrm{nM}$. The sensor detects E2 with a detection limit comparable to 
previously reported sensors using electrochemical $\left(0.1 \mathrm{nM}\right.$, Kim et al. ${ }^{\square}$ and 2.1 $\mathrm{nM}$, Olowu et al.,, 121 ) and optical ( $2.1 \mathrm{nM}$, Yildirim et al.,,$\left.\frac{122}{}\right)$ signal transduction methods applied to a different $\mathrm{E} 2$ aptamer with a $K_{\mathrm{D}}$ of $130 \mathrm{nM} .^{\mathrm{7}}$

The specificity of the sensor using E2 aptamer was examined over a wide range of potential interfering compounds. Fig. 2.9p shows that the aptamer does not discriminate between $\mathrm{E} 2$, progesterone ( $\mathrm{P} 4)$ and testosterone $(\mathrm{T})$ which are from the same general family of steroids with only minor variations in the main molecular structure (refer to the structures of detected compounds Fig. 2.9k). T differs from $\mathrm{E} 2$ by the replacement of $\mathrm{OH}$ at position 2 with a ketone and an extra methyl group at position 10. $\mathrm{P} 4$ differs from $\mathrm{T}$ by the replacement of $\mathrm{OH}$ at position 17 with an ester $\left(\mathrm{COCH}_{3}\right)$. However, excellent discrimination was achieved against molecules that do not belong to this steroidal family but may elicit similar biological response (e.g. bisphenol A (BPA) and 4, 4'-methylenediphenol (BPF)), even when these are mixed with the target molecule, E2. This indicates that the aptamer has a general specificity for a class of molecules belonging to a common steroidal family and shows the applicability of the sensor to detect other small molecules.

\subsubsection{Generality of the sensor}

It was confirmed that the size based scheme is general for the detection of any small molecular target as long as the desired aptamer is available and undergoes reportable conformational change. The adenosine aptamer reported by Kim et al., $\frac{123}{123}$ which is a 27 -mer oligonucleotide with a dissociation constant of $6 \mu \mathrm{M}$ for a 1:2 complex (aptamer : adenosine $)^{48}$ and is one of the most commonly studied small molecule-binding aptamers. The adenosine aptamer was coupled to $217 \mathrm{~nm}$ NPs following the same protocol used to couple the E2 aptamer. The $\zeta$ potential of the bare NPs decreased from $-36.0 \pm 0.1$ to $-40.0 \pm 0.7 \mathrm{mV}$, confirming a successful coupling. Then, an increase of approximately $10 \mathrm{~nm}$ in diameter using TRPS was resolved, as shown in Fig. 2.10a. The smaller increase compared with the E2 aptamer (75-mer) was expected owing to the shorter length of the adenosine aptamer (27-mer). The increase in diameter was not associated with a change in the particle duration FWHM as shown in Fig. 2.10 b: this is likely due to the increased steric drag effect being offset by the increased electrophoretic mobility. As shown in Fig. 2.10a and Fig. 2.10 b, the sensor is able to detect adenosine at levels as low as $500 \mathrm{nM}$ under the same buffer conditions and nanopore configuration as applied for the detection of E2. Consistent with the E2 sensor results, the detection of adenosine was confirmed via a decrease in size for the NP-adenosine 
aptamer conjugate (from 227 to $221 \mathrm{~nm}$ ) and an increase in the particles' FWHM duration (from 0.43 to $1 \mathrm{~ms}$ ). Both of these measured changes exceed the experimental uncertainty indicated by the bin size. This therefore demonstrates the more general applicability of this sensor system for any small molecule. Additionally, the sensor detects adenosine at approximately one order of magnitude lower concentration than what was reported by Kim et al. using an electrochemical aptasensor. $\frac{123}{12}$
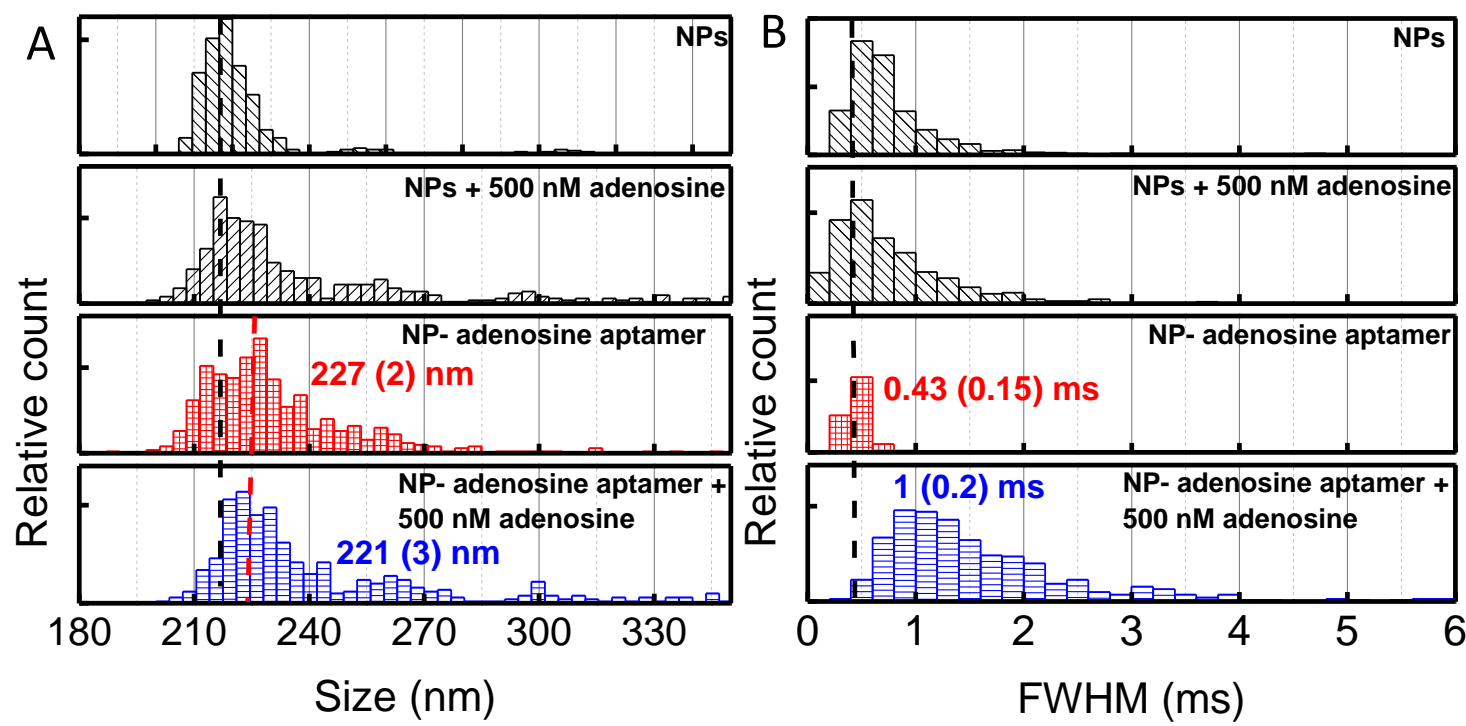

Figure 2.10: a) Size histograms of different samples obtained by TRPS during the detection of $500 \mathrm{nM}$ adenosine. b) FWHM histograms of different samples obtained by TRPS for the same sets of samples. The dashed vertical lines are aligned to the modal bars of the bare NPs (black) and NP-aptamer (red). The bin sizes are $3.5 \mathrm{~nm}$ and $0.2 \mathrm{~ms}$ for size and duration (FWHM) respectively. Numbers in parentheses indicate the standard deviation of the mean of three measurements.

\subsection{Chapter conclusion}

A new aptasensor scheme to detect small molecules of environmental and medical importance such as the reproductive class including the estrogenic family of steroids was developed. The sensor features an E2 aptamer covalently tethered to polystyrene NPs as the signal carrier. It is shown that simple methods for measuring NP size and surface potential can be used to register binding events when the aptamer adopts a more tightly folded conformation around its target. The sensor is able to detect E2 at the lower nanomolar concentration range in buffered wa- 
ter. Importantly, the ability to couple conformational switching to binding signals does not require explicit knowledge of the bound and unbound aptamer conformations. It is only required that binding induces some kind of conformational change - a rather general feature of small molecule binding aptamers since they achieve a compact folded structure with a specific binding pocket. Therefore the sensor design is broadly applicable to other aptamers targeting small molecules as it was demonstrated with adenosine aptamer. Moreover, since signal transduction is achieved without the need to engineer additional features in the aptamer, sequences can be refined purely with the goal of optimizing the binding affinity and selectivity for a particular application. The simplicity of the developed scheme could make it useful not only for sensing applications, but also for screening the affinity of different aptamer sequences during the aptamer evolution process.

\subsection{Experimental section}

Chemicals and Materials used in this Chapter can be found in Appendix D.

\section{Buffers and solutions}

Binding washing buffer (BWB): $2 \mathrm{mM}$ 2-Amino-2-hydroxymethyl-propane-1,3diol (Tris- $\mathrm{HCl}$ ) at $\mathrm{pH} 7.5$ (containing $10 \mathrm{mM} \mathrm{NaCl}, 0.5 \mathrm{mM} \mathrm{KCl}, 0.2 \mathrm{mM} \mathrm{MgCl}$, $0.1 \mathrm{mM} \mathrm{CaCl}_{2}$ and $5 \%$ ethanol $)$ was prepared $(\mathrm{I}=13.26 \mathrm{mM}, \mathrm{pH} 7.5$, resistivity $0.848 \Omega \mathrm{m}$, viscosity $0.891 \mathrm{mPa}$, and relative permittivity 78.5). 0.1\% IGEPAL ${ }^{\circledR}$ was added for TRPS experiments for wetting purposes.

Hybridisation buffer: $0.1 \mathrm{M}$ 2-(N-morpholino) ethanesulfonic acid (MES) at $\mathrm{pH}$ 6.2 was prepared ( $\mathrm{I}=0.1 \mathrm{mM}$, viscosity $1.00 \mathrm{mPa} \mathrm{s}$, relative permittivity 78.5). EDC/NHS solution was prepared in MES at concentrations of $0.01 \mathrm{M}$, and this solution was kept at $4{ }^{\circ} \mathrm{C}$ for a week and then discharged. Stock $100 \mu \mathrm{M}$ ethanol solutions of E2 and the interfering agents were prepared, and the detected concentrations were prepared in BWB, with the ethanol quantity held constant at 5 $\%$. NPs were suspended in MES buffer at a concentration of $5.2 \times 10^{10}$ particles $\mathrm{mL}^{-1}$. 


\section{NP functionalization}

NP functionalization: $400 \mu \mathrm{L}$ of carboxylated NPs $\left(5.2 \times 10^{10}\right.$ particles $\left.\mathrm{mL}^{-1}\right)$ in MES were sonicated for 10 minutes, following which $200 \mathrm{nmol}$ of EDC and NHS (20 $\mu \mathrm{L}$ of $0.01 \mathrm{M}$ solution of both in MES) was added and incubated for $40 \mathrm{~min}-$ utes. $0.1 \mathrm{nmol}$ of aptamer was added after being denatured at $70{ }^{\circ} \mathrm{C}$ for $5 \mathrm{~min}$ utes and the reaction was incubated overnight in plastic centrifuge vials at room temperature. The sample was centrifuged twice at 14,000 rpm for 25 minutes (MIKRO 120- Hettich was used in all centrifugation experiments), with washing and final suspension in MES or BWB (with or without IGEPAL ${ }^{\circledR}$ ) depending on the purpose of the experiment. Finally, the sample was sonicated for $5 \mathrm{~min}$ utes before performing a sensing experiment. It should be noted that there is an approximately $11 \%$ loss of particles from the centrifugation process. This was determined via measuring the fluorescence of F-aptamer-NP before and after two cycles of centrifugation at 14,000 rpm for 25 minutes.

\section{Scanning electron microscope (SEM)}

SEM images were conducted using $20 \mu \mathrm{L}$ of bare and functionalised NPs (5.2 x $10^{10}$ particles $\mathrm{mL}^{-1}$ ) in deionized water (Milli-Q, $18.2 \mathrm{M} \Omega \mathrm{cm}$ ). These samples were deposited on a silicon wafer and dried under vacuum prior to graphite dust coating. JEOL JSM-6500 operated at an accelerating voltage of $50 \mathrm{kV}$ was used for the SEM imaging.

\section{Sensing experiments}

General protocols: In the case of TRPS measurements, $90 \mu \mathrm{L}$ of NP-aptamer in BWB $\left(5.2 \times 10^{10}\right.$ particles $\left.\mathrm{mL}^{-1}\right)$ was mixed with the appropriate volume of E2 in BWB stock solution ( $1 \mu \mathrm{M}$ or $2 \mu \mathrm{M})$ to obtain different E2 concentrations in a total volume of $100 \mu \mathrm{L}$ of BWB. The samples were then incubated for 20 minutes at room temperature to facilitate binding between E2 and the aptamer. The specificity experiments were done following the same procedure described for the detection of E2 and using $50 \mathrm{nM}$ of each interfering agent. The nanopore membrane was immersed in $20 \mathrm{~mL}$ deionized water (Milli-Q, $18.2 \mathrm{M} \Omega \mathrm{cm}$ ) containing 1 gram of activated charcoal and then rinsed with ethanol before any specificity examination. In the case of control experiments without E2 or without coupling agents, it was ensured that the composition of the solution was identical in every other 
way. The same procedure was followed for the measurements of size in DLS and $\zeta$-potential in phase analysis light scattering except that the final volumes of the samples were $1 \mathrm{~mL}$. Samples were sonicated for 5 minutes before TRPS or DLS measurements to ensure that the NPs were well dispersed.

TRPS measurements: For all measurements, a tunable pore membrane (size designation NP200) was mounted on a qNano instrument (both were obtained from Izon Science). The membrane was stretched until the desired pore size was reached, in this case with the teeth of the device separated by $46.11 \mathrm{~mm}$. $75 \mu \mathrm{L} \mathrm{BWB}$ with no detergent was placed in the lower fluid cell and $40 \mu \mathrm{L}$ BWB with 0.1 $\%$ IGEPAL $^{\circledR}$ detergent was placed in the upper cell. The presence of IGEPAL ${ }^{\circledR}$ in the BWB was crucial to facilitate smooth NP-aptamer transit in TRPS (see Fig. A.1). An external voltage of $0.26 \mathrm{~V}$ was applied between the electrodes in the upper and lower fluid cells to give a background current (I) of $70 \pm 10$ nA. 40 $\mu \mathrm{L}$ of the test sample was placed in the upper fluid cell, ensuring that no bubbles were present. At least 500 blockade events were collected (which generally took 4-7 minutes) and analysed using proprietary event analysis software from Izon Science (v. 2.2). Sizes were calculated as reported in Vogel et al. ${ }^{100}$ and described in Chapter 2(Section 2.3) after calibration with an appropriate standard sample, typically the carboxylated polystyrene NPs with a diameter of $217 \mathrm{~nm}$. With regard to this study, the samples were run in TRPS and followed by calibration with $217 \mathrm{~nm}$. The software provided with the qNano automatically plots the size and FWHM distributions. The bin size was reduced to $3.5 \mathrm{~nm}$ for the size histograms and $0.2 \mathrm{~ms}$ for the FWHM distributions. The reported modes are the values located at the centres of the mode bins. This indicates that there is an inherent uncertainty of $1.75 \mathrm{~nm}$ and $0.1 \mathrm{~ms}$ associated with mode values reported for the sizes and FWHM respectively.

DLS and $\zeta$-potential measurements: A Zetasizer Nano ZS (back scattering mode, Malvern Instruments, UK) with a $633 \mathrm{~nm}$ red laser was used. The instrument provides both size and $\zeta$-potential values with no need for a reference calibration. 1 $\mathrm{mL}$ of each nanoparticle sample was placed in a $1 \mathrm{~cm}$ path length disposable cuvette for size measurement. For $\zeta$-potential measurement, each sample was used to fill a folded capillary cell until the Au-electrodes were covered and no air bubbles were present in the cell. The cell was inserted in Zetasizer and equilibrated at $25{ }^{\circ} \mathrm{C}$ for 2 minutes prior to either size or $\zeta$-potential measurement. 


\section{Spectroscopy and aptamer characterisation}

Conformational transition studies by the $\mathrm{CD}$ technique were undertaken using a Chirascan CD spectrometer instrument. $1 \mathrm{~mL}$ aliquots of $400 \mathrm{nM}$ solutions of E2 aptamer were made in BWB and independently contained different concentrations of $\mathrm{E} 2(0,0.5,3.5$ and $6.5 \mu \mathrm{M})$. A standard $1 \mathrm{~cm}$ path length quartz cell was used to measure the CD spectra over the wavelength range from 200 to $400 \mathrm{~nm}$ and the scanning speed was $200 \mathrm{~nm}$ per minute. It should be noted that the CD spectra were recoded as the difference in absorption, $\Delta \mathrm{A}$, and were converted to elipticity (in mdegree) by multiplying with 32.98 .124

Fluorescence experiments for the aptamer coverage density were done by taking different NP concentrations $\left(1.04 \times 10^{10}, 2.08 \times 10^{10}, 3.12 \times 10^{10}\right.$ and 4.16 $\times 10^{10}$ particles $\mathrm{mL}^{-1}$ ) in stock solution and applying the previously mentioned coupling reaction but with the fluorescently-tagged aptamer (F-aptamer). After incubation and purification, the NP-aptamer samples were suspended in 500 $\mu \mathrm{L}$ MES buffer and the supernatant solutions were made up to $1 \mathrm{~mL}$. The calibration curve was established by measuring the fluorescent intensity of different F-aptamer concentrations made in $500 \mu \mathrm{L}$ MES buffer samples containing $2.08 \times$ $10^{10}$ particles $\mathrm{mL}^{-1}$ of carboxylated NPs to account for any interference caused by scattering from the NPs. A standard $1 \mathrm{~cm}$ path length quartz fluorescence cell was used to measure the emission spectra over the wavelength range from 650 to $800 \mathrm{~nm}$, with excitation at $645 \mathrm{~nm}$. A $665 \mathrm{~nm}$ long pass filter was used to block scattered excitation light. The peak intensities at $700 \mathrm{~nm}$ were used for quantifying concentrations. Fluorescence experiments were performed with a Shimadzu RF-5301PC spectrofluorophotometer. Excitation and emission slit widths were set to the relatively low resolution of $10 \mathrm{~nm}$ and $15 \mathrm{~nm}$, respectively, in order to enhance the measured fluorescence intensities in such dilute samples.

$K_{\mathrm{D}}$ experiments were conducted using NP-aptamer conjugates to isolate the bound and unbound fractions of E2. $0.1 \mathrm{nmol}$ of E2 aptamer was functionalised to 217 $\mathrm{nm}$ particles as described previously. After the purification steps the NP-aptamer, samples were served as semi-dry form for E2 solutions. Serial concentrations of E2 (5 nM up to $400 \mathrm{nM}$ ) in BWB were made in $1 \mathrm{~mL}$ and incubated independently with NP-aptamer (making the aptamer concentration $100 \mathrm{nM}$ ) overnight at room temperature. After that, the samples were centrifuged twice at $14000 \mathrm{rpm}$ for 30 minutes. Then the unbound fraction (supernatant) was quantified via the measurements of the emission of E2. E2 can be measured via UV-fluorescence spectroscopy since E2 exhibits a characteristic emission band at $310 \mathrm{~nm}^{[125}$ and can be reliably detected as low as $5 \mathrm{nM}$ concentration, as shown in Fig. 2.4. A standard 1 
$\mathrm{cm}$ path length quartz fluorescence cell was used to measure the emission spectra of E2 over the wavelength range from 280 to $400 \mathrm{~nm}$, with excitation at $279 \mathrm{~nm}$. The peak intensities at $310 \mathrm{~nm}$ were used for quantifying concentrations. Then, unbound fraction of $E 2\left(f_{a}\right)$ was calculated. $1 / f_{a}$ was plotted against 1/[E2] of the original E2 concentrations as shown in Fig. 2.5. There was a saturation offset at 0.72 occurring probably due to the loss of the NP-aptamer conjugate during the centrifuge and samples handling processes. Therefore, the data was normalised before subsequent $K_{\mathrm{D}}$ calculation using Eq. 2.5. Fluorescence experiments were performed with a Shimadzu RF-5301PC spectrofluorophotometer. Excitation and emission slit widths were set to the relatively low resolution of $10 \mathrm{~nm}$ in order to enhance the measured fluorescence intensities in such dilute samples.

Equilibrium filtration experiments were done using 3K nitrocellulose filters (Amicron Ultra - $0.5 \mathrm{~mL}$ 3K from Sigma-Aldrich). Serial concentrations of E2 in $1 \mathrm{~mL}$ BWB $(5-400 \mathrm{nM})$ were filtered using the $3 \mathrm{~K}$ nitrocellulose filters by centrifugation at 14,000 rpm for 12 minutes and measurement of fluorescence of E2 as previously described.

Dot blotting experiments were done by Dr. Shalen Kumar during the generation of the 75-mer aptamer by SELEX. Full experimental details can be found in the published work. ${ }^{[23}$ Briefly, nitrocellulose membranes were used to immobilise several amounts of E2 in ethanol. After blocking steps, biotin-aptamer was incubated individually with the membranes containing different amounts of E2 for overnight. The bound E2-aptamers were visualised using chemoluminescence where a secondary incubation with streptavidin-peroxidase conjugate was necessary. 


\section{Chapter 3}

\section{Colorimetric aptasensor}

\subsection{Abstract}

This chapter describes a strategy enabling ultrasensitive colorimetric detection of E2 in water and urine samples using DNA aptamer-coated AuNPs. Starting from an established sensor format where aggregation is triggered when targetbound aptamers dissociate from AuNP surfaces, it was demonstrated that stepchange improvements are easily accessible through deletion of excess flanking nucleotides from aptamer sequences. After evaluating the lowest energy twodimensional configuration of the 75-mer aptamer, new 35-mer and 22-mer aptamers were generated with $K_{\mathrm{D}}$ 's of $14 \mathrm{nM}$ and $11 \mathrm{nM}$ by simply removing flanking nucleotides on either side of the inner core. The shorter aptamers were found to improve discrimination against other steroidal molecules and to improve the colorimetric detection limit for E2 to $200 \mathrm{pM}$ (25-fold). It was found that the excess flanking nucleotides suppress signal transduction by causing target-bound aptamers to remain adhered to AuNPs, which was confirmed via surface sensitive electrochemical measurements (chronocoulometry). However, comparison between the 22-mer and 35-mer systems show that retaining a small number of excess bases is optimal. In fact, this simple strategy enabled facile detection of E2 in urine at $5 \mathrm{nM}$, approaching levels of biological relevance. 


\subsection{Introduction}

Of the different types of aptasensors available, those exploiting AuNP aggregation are emerging one of the most effective and easily implemented methods. $\frac{38}{3}$ As shown in Scheme 3.1, the attractive interaction of nitrogen bases with Au surfaces ${ }^{16}$ leads to a dispersion of negatively charged DNA-coated AuNPs that are stable in moderate salt concentrations.

Binding of the target molecule induces a conformational change in the DNA aptamer ${ }^{9 / 31}$ which in most cases reduces its affinity to AuNPs and thereby destabilizes the dispersion towards salt. $\frac{38}{}$ At an optimised ionic strength (defined as $I=1 / 2 \sum C_{i} Z_{i}^{2}$ ) for sensing, presence of the target is identified by the color change resulting from aggregation of AuNPs that are no longer protected by aptamers. The degree of aggregation is sensitive to the concentration of the target molecule: this can be quantified by UV-visible absorption and is also evident to the naked-eye. $22-44[115] 127$ This sensing method essentially exploits the differential affinity of the aptamer towards the AuNP surface versus the target. Interaction with the AuNP is non-specific and can include the entire aptamer sequence. In contrast, the aptamer-target interaction occurs in a specific binding domain that might be only a fraction of the aptamer sequence for small molecule targets. Excess bases not directly committed to the binding domain may still interact with the AuNPs when such a target is bound, ultimately suppressing signal transduction by interfering with the complete dissociation of target-bound aptamers from AuNPs. It was hypothesized that eliminating excess bases will result in enhanced sensitivity in AuNP aggregation sensors, as depicted in the comparison on long and short aptamer responses in Scheme 3.1 .

DNA aptamer sequences commonly include bases that are redundant to target binding. ${ }^{127128}$ Aptamers are generated comprising a random core sequence to provide the diversity needed for selection, as well as flanking primers for amplification by polymerase chain reaction. ${ }^{31}$ Moreover, since the synthetic evolution process selects for affinity, there is little evolutionary pressure to eliminate redundancies (i.e., SELEX does not remove non-binding nucleotides) that do not affect affinity. Many small molecular aptasensors have been developed with the original primers retained. ${ }^{23 / 126}$ In several instances, sequences have been modified through insertions or deletions outside of the core region but without noticeable perturbations of their affinities. $\frac{48 / 49}{}$ While excess bases may be innocent with respect to target binding affinities, their effect on signal transduction is expected to depend on the sensor format used. 


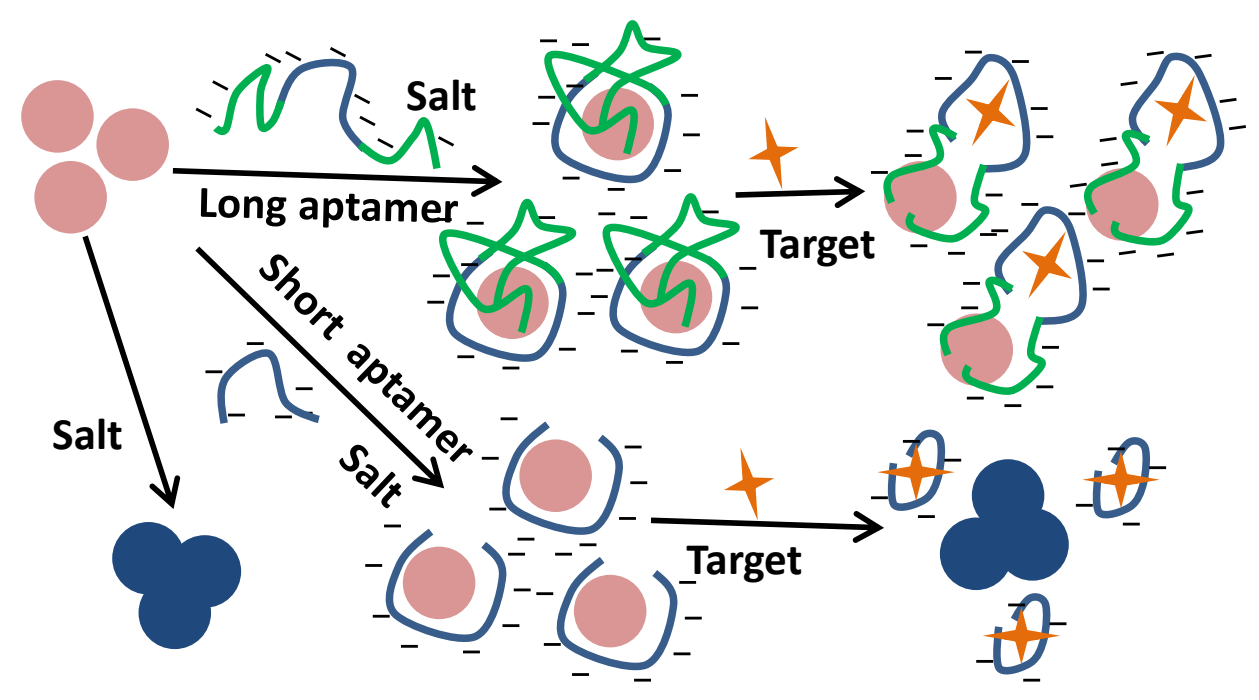

Scheme 3.1: Overview of the AuNP aggregation based aptasensing method. The scheme highlights the proposed suppression of target binding signals with the long aptamer when bases outside of the binding domain (green) remain adhered to the AuNPs, preventing aggregation.

Consistent with the proposed hypothesis, several noteworthy AuNP aggregation sensors achieve high performance using truncated versions of the parent aptamer sequences. For example, the central 21-mer stem loop from the kanamycin binding aptamer has a comparable $K_{\mathrm{D}}(78.8 \mathrm{nM})$ as the parent $85-$ mer $(85.6 \mathrm{nM})$ and results in $25 \mathrm{nM}$ level of detection in the AuNP format. $\frac{127}{12} \mathrm{~A}$ 20-mer stem loop binding region was also identified within a 91-mer aptamer for binding acetamiprid $\left(K_{\mathrm{D}} 498 \mathrm{nM}\right)^{128}$ and used in truncated form with AuNPs to achieve $5 \mathrm{nM}$ detection in soil samples. ${ }^{129}$ Similarly, AuNP based sensors for bisphenol $\mathrm{A}^{43}$ and ochratoxin $\mathrm{A}^{444}$ achieve $400 \mathrm{pM}$ and $20 \mathrm{nM}$ detection, respectively, using sequences excluding the regions flanking the putative binding domains in the original aptamers. However, none of these studies have directly addressed the proposed hypothesis through comparing the sensitivity of truncated versus original extended sequences using the same AuNP sensor scheme. Nor have previous studies directly probed how flanking nucleotide sequences affect surface interactions with the AuNPs. While the vast majority of AuNP aggregation sensors depend on target triggered dissociation from AuNP surfaces, systematic investigations of aptamer length have only been carried out for less common formats where the target triggers cross-linking ${ }^{38}$ or a conformational change to the surface-bound aptamer that suppresses aggregation. 130

In this research, the effect of flanking nucleotide sequences in a AuNP based colorimetric sensor was directly resolve for detecting E2 in water and urine samples. It was found that by simply eliminating the flanking 20 or more nucleotides on 
either side of a core sequence yields a 25-fold improvement in sensitivity from $5 \mathrm{nM}$ to $200 \mathrm{pM}$. The improvement of the 35-mer and 22-mer is only partially related to improved affinity for E2 ( $K_{\mathrm{D}} \mathrm{s} 14$ and $\left.11 \mathrm{nM}\right)$, and also results in improved discrimination against structurally similar molecules. Surface sensitive electrochemical techniques were used to confirm that suppressed colorimetric signal transduction for the longer 75-mer aptamer is a direct result of the residual affinity that target-bound aptamers have towards AuNPs. However, close comparison of the 35-mer with the minimal 22-mer shows that retaining a small number of excess bases is optimal.

\subsection{Results and discussion}

\section{Development of colorimetric E2 sensor with the 75-mer aptamer}

The investigation started with applying the AuNP aggregation assay illustrated Scheme 3.1 to the previously studied 75-mer E2 aptamer sequence in Chapter 2. The aptamer has a $K_{\mathrm{D}}$ of $25 \mathrm{nM}$. The colorimetric measurements were complemented with $\zeta$-potential measurements to probe how different states of the aptamer perturb the AuNP surface properties. After mixing the 75-mer E2 aptamer $(33.3 \mathrm{nM}$ ) with AuNPs, the $\zeta$-potential of the AuNPs decreased from -23.5 $\mathrm{mV}( \pm 0.3)$ to $-40.2 \mathrm{mV}( \pm 0.9)$ (Table 3.1), thereby confirming successful adsorption of the poly ionic aptamer, and suggesting greater tolerance towards salt. This was confirmed via a titration with salt, as shown in Fig. 3.1, where aggregation is evidenced by the reduced relative absorption of the unaggregated AuNP surface plasmon peak $523 \mathrm{~nm}$. The amount of aptamer for E2 sensing was also optimised to be 33.3 nM 75-mer (or aptamer:AuNP of 3:1) for $14 \mathrm{nM}$ of AuNPs (Fig. 3.1p).

Optimal aptamer:AuNP stoichiometric ratios used in the past vary widely from only $1: 1$ or less ${ }^{43 / 127}$ to over 50:1. ${ }^{1261130}$ This wide variation relates to different sizes of AuNPs, as well as the sequence dependent affinities of aptamers to AuNPs. Their intrinsic affinity is highly sensitive to the base composition, and is substantially higher for sequences like the 75-mer where most bases exist freely in loop regions (see Fig. 3.4) than for those where most bases are bound in double-stranded stem regions. ${ }^{[38}$ Fig. 3.1 p shows that in this study, higher aptamer coverage results in weaker sensitivity to salt, and a less sharp transition to aggregation. This excess protection towards aggregation buffers the AuNPs towards target-induced aggregation at higher aptamer coverage. 

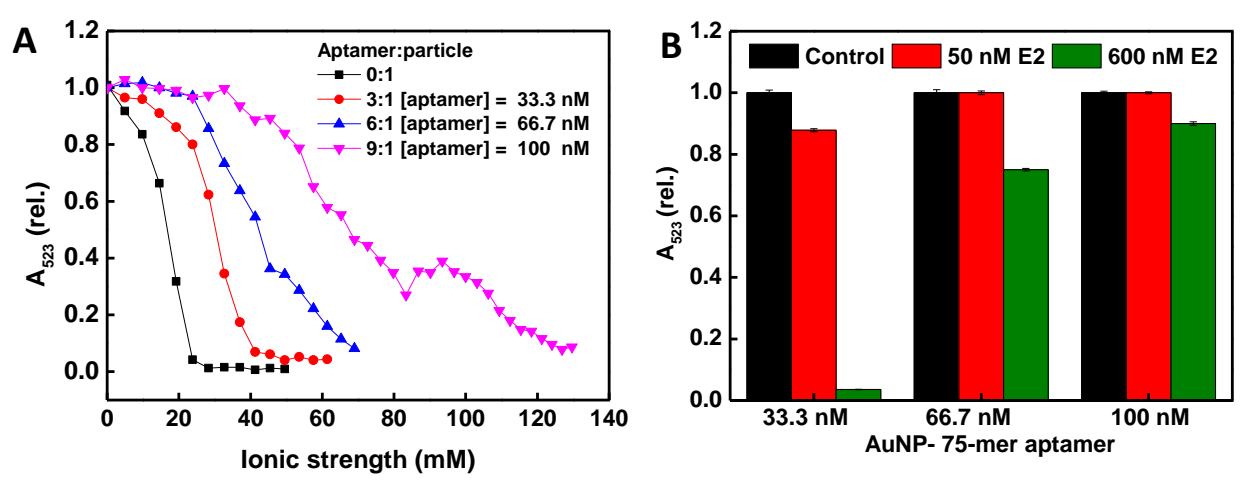

Figure 3.1: a) Salt titration of different concentrations of the 75-mer aptamer. b) Sensitivity examination of various concentrations of the 75-mer aptamer with E2 concentrations. Optimised salt concentration was used for each case as detailed in the Experimental Section.

Fig. 3.3a shows that after incubating with $100 \mathrm{nM}$ E2, the aptamer-coated AuNPs lost resistance towards salt, confirming that at least some of the aptamers dissociate from the AuNP surface when binding to E2. This conclusion is supported by the less negative $\zeta$-potential $(-32.3 \mathrm{mV}( \pm 0.9))$ measured for the AuNP-75-mer after incubation with $1000 \mathrm{nM}$ E2. Fig. 3.3a also reveals the optimal salt concentration $(23.8 \mathrm{mM})$ for E2 detection, whereby the AuNP-75-mer dispersion is on the edge of stability and where the introduction of E2 triggers significant aggregation.

Fig. 3.3 shows colorimetric detection for E2 in the nanomolar range and nakedeye detection above $100 \mathrm{nM}$ (top panel), where binding induced aggregation turns the pink/red colloidal solution to purple/blue. The series of absorption spectra (Fig. 3.3p) confirmed increasing aggregate absorption at $625 \mathrm{~nm}$ at the expense of the unaggregated plasmon peak at $523 \mathrm{~nm} \cdot \stackrel{36}{ }$ Transmission electron microscopy (TEM) and dynamic light scattering (DLS) measurements (Fig. 3.2) also provided qualitative support for E2-induced aggregation, although it should be noted that the dry samples used for TEM are subject to additional aggregation forces. The lowest colorimetrically detected concentration of E2 using the 75-mer aptamer was $5 \mathrm{nM}$, with good linearity $\left(\mathrm{R}^{2}=0.92\right)$ over the range from $5 \mathrm{nM}$ to $400 \mathrm{nM}$, before saturation occurs.

It was confirmed that the colorimetric signals arose from a specific interaction with the aptamer by observing no colorimetric response when incubating E2 with bare AuNPs and with AuNPs coated with a random 75-mer. This random sequence (given in Appendix D has same total base composition as the 75-mer aptamer but its bases are arranged randomly, thereby eliminating the specific 

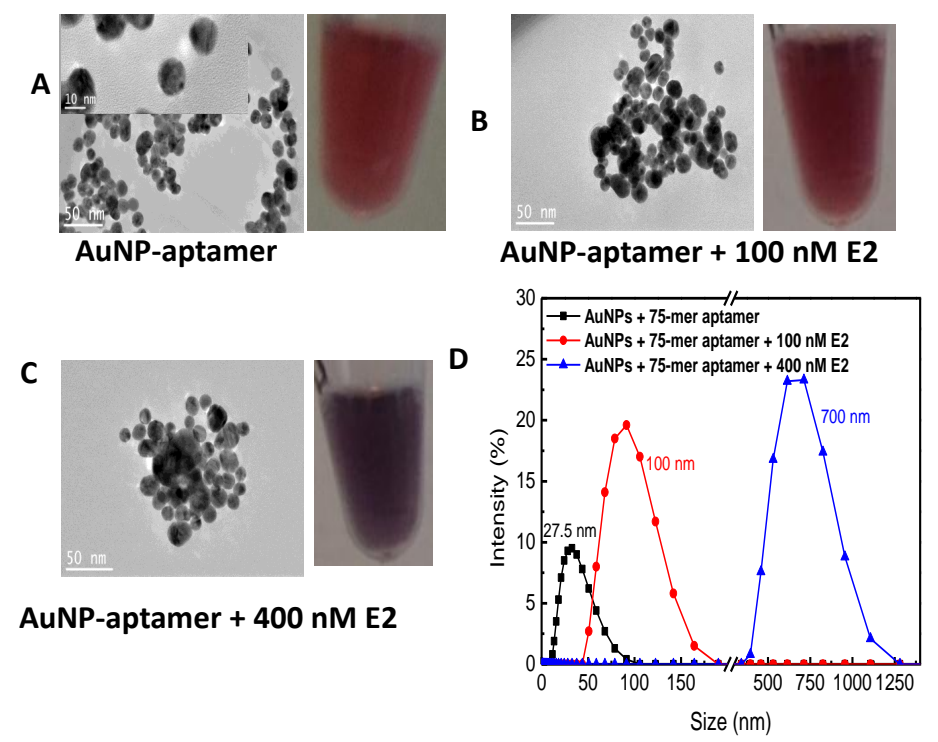

Figure 3.2: TEM images (left) and photographs (right) of a) AuNP- 75-mer aptamer, and the detection of b) $100 \mathrm{nM}$ and c) $400 \mathrm{nM}$ E2. d) DLS size characterisation for the same set of samples (average size distribution is indicated in the figure).

target-binding domain that evolved in the 75-mer aptamer. The less $\zeta$-potential and increased salt resistance compared with bare AuNPs confirmed that the random sequence still coats the AuNPs, however incubation with E2 did not produce further changes (refer to Table 3.1 and Fig. 3.3a).

Having established sensitivity towards E2, the ability of the sensor to discriminate against structurally similar molecules was examined with progesterone (P4) and testosterone (T), which belong to the same steroidal family class as E2, and non-steroidal compounds such as BPA and BPF (structures are shown in Fig. 2.9 in Chapter 2). These compounds were individually exposed to the AuNP-75-mer colloidal solutions at $200 \mathrm{nM}$ target concentrations, where E2 exhibits a strong response (top panel of Fig. 3.3 d). The 75-mer aptamer showed good discrimination against the non-steroidal molecules (BPA and BPF), however, $\mathrm{T}$ triggered a $33 \%$ response and $\mathrm{P} 4$ a $45 \%$ response relative to $\mathrm{E} 2$ (Fig. $3.3 \mathrm{~d}$, raw spectra are provided in Appendix B, Fig. B.2). The present specificity results are broadly consistent with what was concluded from the size based sensor, Fig. 2.9 in Chapter 2. where the 75-mer aptamer responded to P4 and T and did not show affinity for $\mathrm{BPA}$ and BPF. 

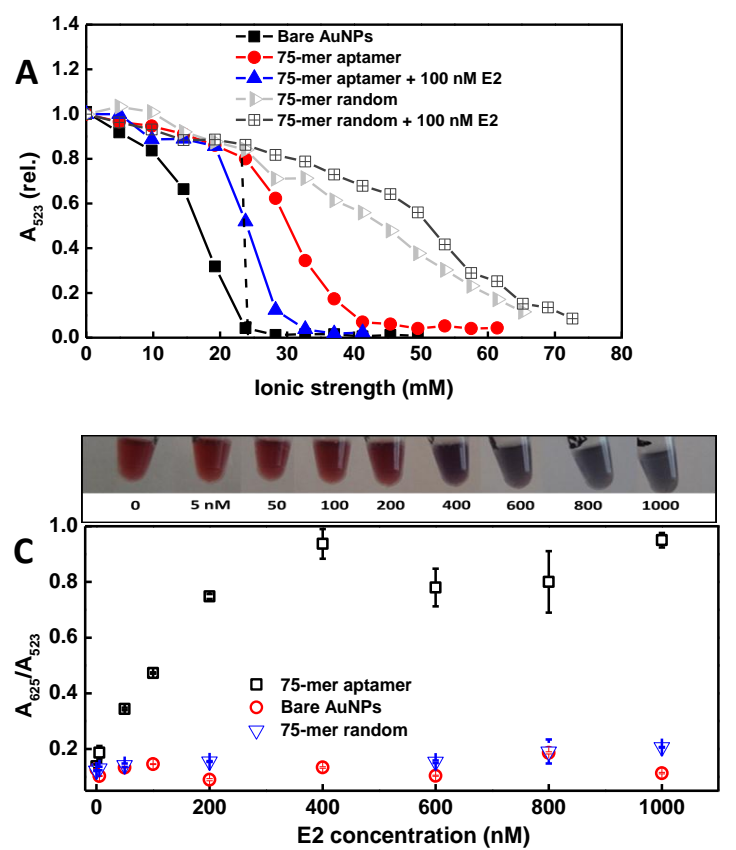
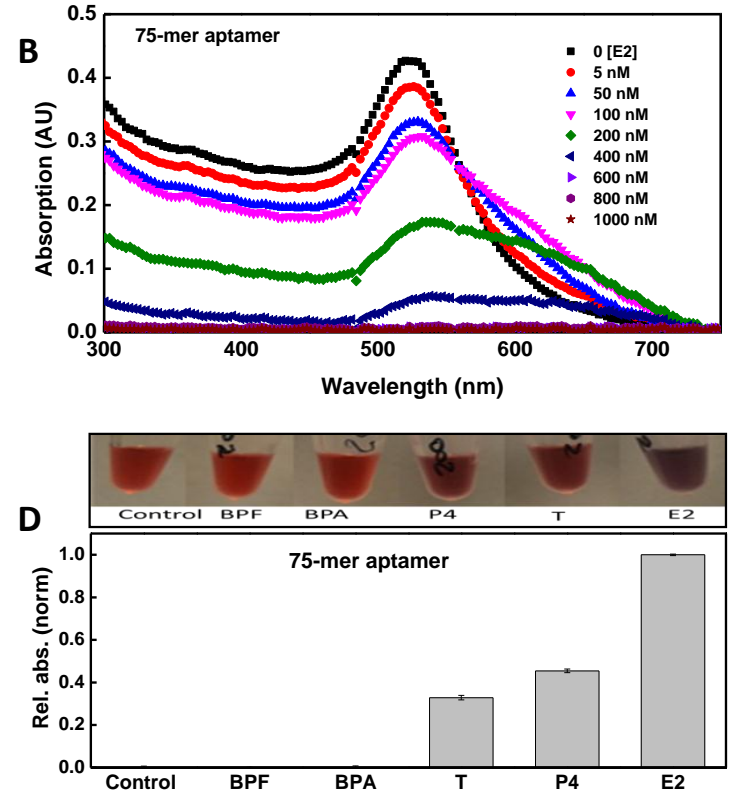

Figure 3.3: a) Salt dependent aggregation of AuNPs, AuNP-75-mer aptamer and AuNP-75-mer aptamer + 100 nM E2 (the same type of data for the 75-mer random DNA is also shown), leading to determination of the optimal salt concentration for sensing (indicated by the black arrow). Aggregation is measured via the relative absorption at $523 \mathrm{~nm}$. b) Absorption spectra of E2 sensing with the 75-mer aptamer. c) Colorimetric aptasensor response towards a range of E2 concentrations using the AuNP-75-mer aptamer compared with bare AuNPs and AuNPrandom-75-mer controls. d) Specificity examinations of interfering molecules (at $200 \mathrm{nM}$ ) using the AuNP-75-mer aptamer colloidal solution. Normalised absorptions at $523 \mathrm{~nm}$ were subtracted from the control and plotted relative to the E2 response. Error bars indicate standard deviation of the mean from three experiments.

\section{Truncation of the 75-mer aptamer and CD characterisation}

Under E2 saturation, neither the colorimetric salt titration curve nor the $\zeta$-potential values returned to those of the bare AuNPs. Residual aptamer molecules remain adhered to the AuNPs under these conditions, conferring electrostatic stability and thus diminishing their potential sensitivity. Two conceivable scenarios are that target-bound aptamers remain adhered to AuNPs via bases outside of the binding region, and that some aptamer molecules are prevented from accessing their target-binding structures when adhered to AuNPs. It was postulated that specific binding occurs in the central stem-loop region of the minimum energy $2 \mathrm{D}$ structure shown in Fig. 3.4a (note that this figure is the same as Fig. 2.3 shown 
in the previous Chapter). The minimum energy 2D structure was predicted by a widely recognised method known as M-Fold, 109 that calculates energy matrices which determine optimal 2D structures (i.e. determining possible base pairing within a sequence). To test how bases outside the central stem-loop region affect signal transduction, the flanking sequences were eliminated as indicated in Fig. 3.4 a. All of the forward and reverse primer sequences (fixed sequences used in SELEX for PCR processes) were eliminated to generate a 35-mer aptamer, and a more aggressively truncated 22-mer aptamer isolates the stem-loop binding region.

To explore the possibility that the flanking primers may also perturb the 3D structure of the core region and thereby enhance or suppress interaction with the target or with interfering molecules, the 3D structures via CD spectroscopy were probed. CD spectroscopy has previously been employed to study 3D structures of homogenous DNA and RNA,,$\sqrt[131132]{2}$ as well as probing conformational changes induced by interactions with the target. ${ }^{23 / 43 \mid 44} \mathrm{~A}$ random ssDNA sequence of a B-DNA form is characterized by ellipticity maxima and minima at $280 \mathrm{~nm}$ and $248 \mathrm{~nm}$, respectively, ${ }^{113}$ which is largely what is observed for the 75-mer aptamer (Fig. 3.4p), although the maximum appears closer to $270 \mathrm{~nm}$. After eliminating the flanking nucleotides, the new 35-mer and 22-mer aptamers showed distinctively different CD spectra. As shown in Fig. 3.4b, the $248 \mathrm{~nm}$ minimum is retained with supressed intensity, but the $270 \mathrm{~nm}$ band splits into two bands at $265 \mathrm{~nm}$ and $295 \mathrm{~nm}$. The observed CD spectra indicates a specific 3D stem-loop structure that may exist for the common region of all three, but whose CD features are obscured in the 75-mer by the long unstructured flanking sequences. It was also found that replacing the flanking 20 base primer sequences with 20-T sequences produced a similar CD spectrum as the 75-mer with primers (Fig. B.4 in Appendix B). Incubating each of the three aptamers with $10 \mu \mathrm{M}$ E2 resulted in very minor changes in the CD spectra (Fig. B.3 in Appendix B which is consistent with previous $\mathrm{CD}$ investigations. ${ }^{124}$ 

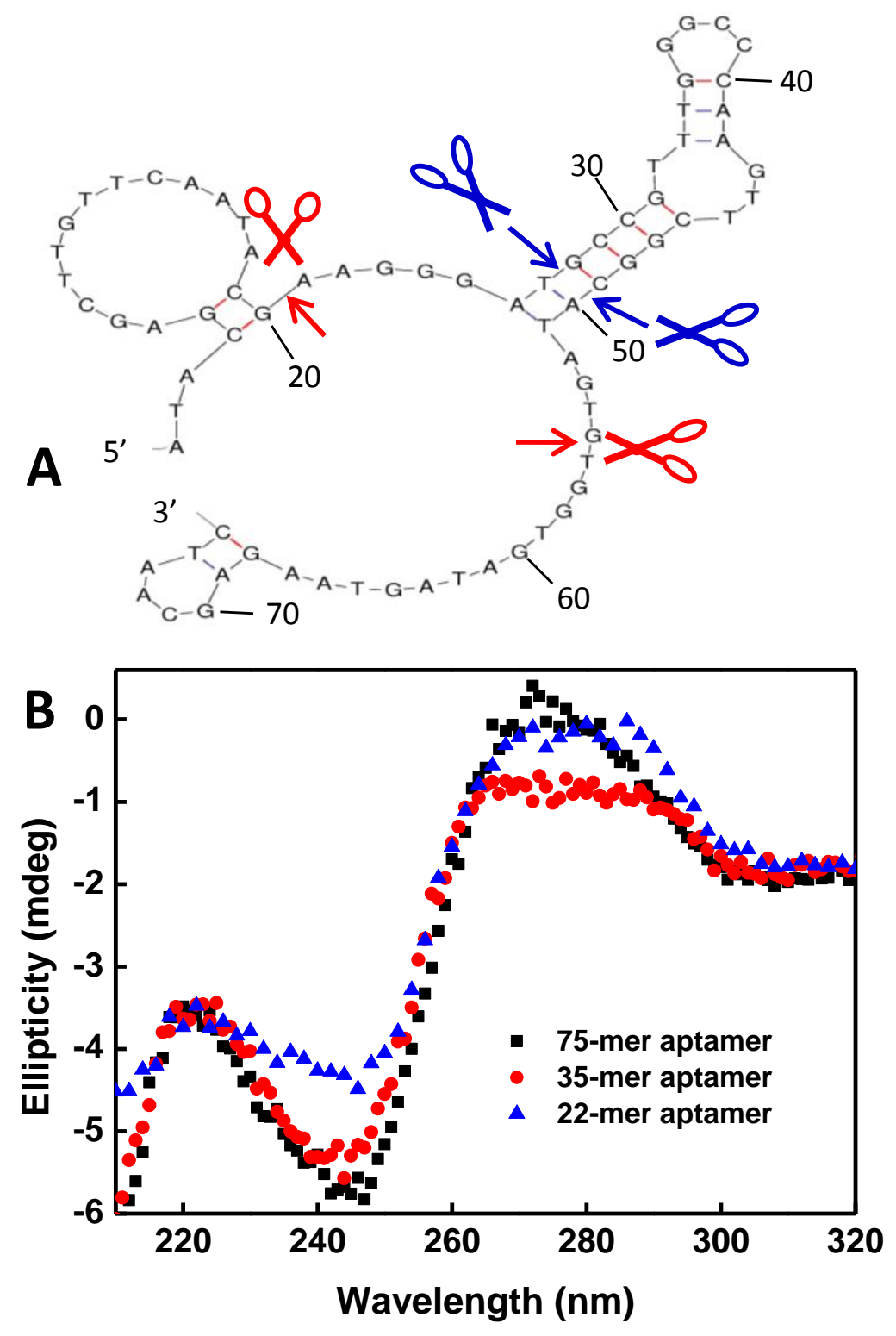

Figure 3.4: a) Secondary structure of the 75-mer aptamer indicating the truncation positions to generate the 35-mer and 22-mer. The secondary structure was predicted by an online web server Mfold ${ }^{109}$ under the following conditions: $25^{\circ} \mathrm{C}$, $10 \mathrm{mM} \mathrm{NaCl}$ and $0.1 \mathrm{MgCl}_{2}$ (BWB conditions). b) CD spectra for $400 \mathrm{nM}$ of the 75-mer and $600 \mathrm{nM}$ of the 35-mer and 22-mer ap-tamers.

$K_{\mathrm{D}} \mathrm{s}$ of the 35-mer and 22-mer aptamers

Using the method that was previously described in Chapter 2 to determine the $K_{\mathrm{D}}$ of the 75 -mer aptamer $(25 \pm 3 \mathrm{nM})$, the $K_{\mathrm{D}}$ of the new 35 -mer $(14 \pm 2 \mathrm{nM})$ and 22-mer (11 $\pm 1 \mathrm{nM}$ ) aptamers with E2 were determined, as shown in Fig.3.5 (more details are provided in Appendix B, Fig. B.9.). Affinity for E2 was retained, even slightly enhanced, upon truncating the flanking sequences, validating the simple 
shortening strategy employed.

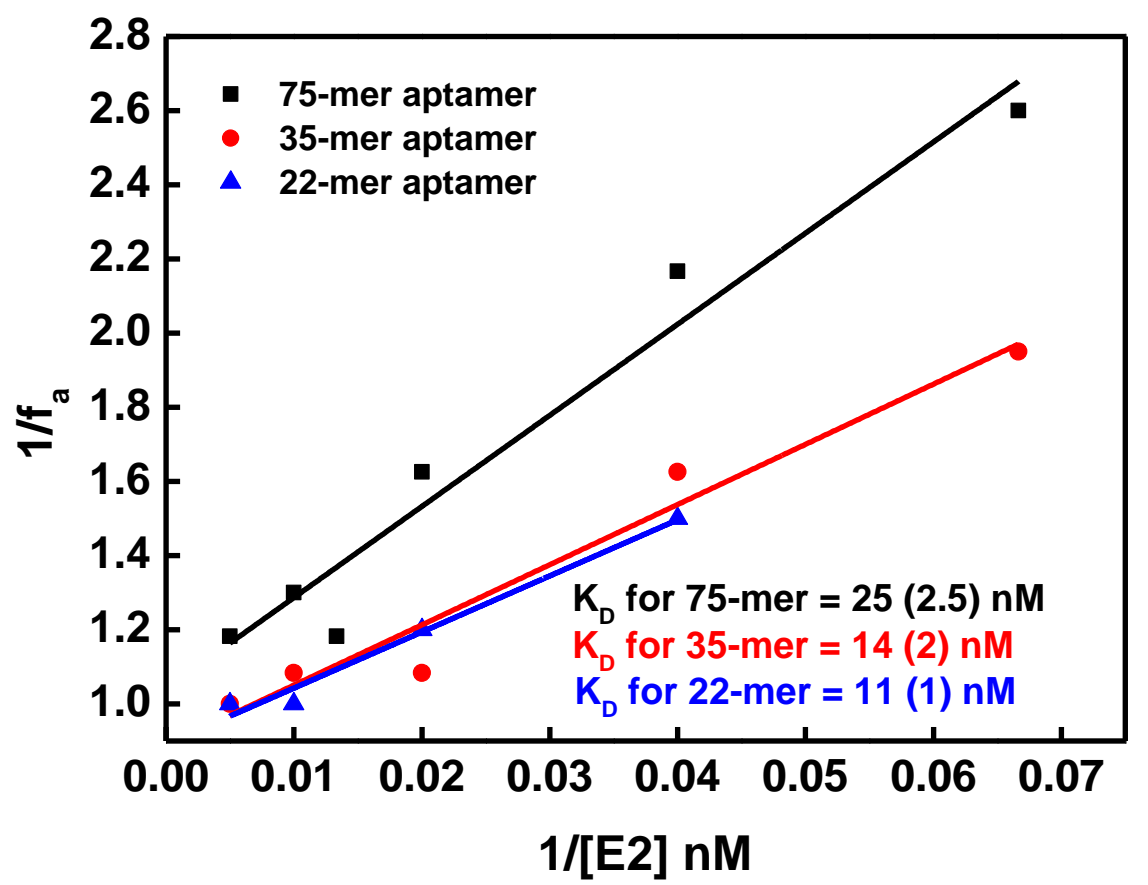

Figure 3.5: Saturation binding curve of E2 with the NP-35-mer, NP-22-mer and NP-75-mer aptamers. Numbers in brackets indicate standard deviation of the fits.

\section{Sensing with the 35-mer and 22-mer aptamers}

Having established that the shorter sequences still bound E2, their interaction with AuNPs was investigated. Adsorption was confirmed by a more negative $\zeta$-potential when bare AuNPs were mixed with either aptamer; from -23.5 mV to $-29 \mathrm{mV}$ for both the 35-mer and the 22-mer (Table 3.1). The smaller difference compared to the 75-mer aptamer (above) may result from a lesser degree of aptamer coverage as well as the lower charge content of the 35-mer and 22mer forms. Interestingly, randomised sequences of each length (including the 75-mer) led to substantially more negative $\zeta$-potential exceeding $-40 \mathrm{mV}$ (Table 3.1) (Sequences are provided in Appendix D). This comparison suggests that the secondary structures adopted by the target-binding aptamers (with substantial double-stranded stem regions) result in less total DNA coverage. This may be due to them occupying a larger surface area than random DNA strands that can be loosely tethered and have the flexibility to pack around each other, in addition to the weaker affinity of double-stranded DNA to AuNPs. The observed variation in $\zeta$-potential is also reflected in salt-titration measurements (Fig. 3.3 a for the 75-mer, Fig. 3.6a for the 35-mer and Fig. 3.7a for the 25-mer) where random 
sequences resulted in a greater salt-resistance in comparison to their respective aptamer sequences.

Table 3.1: $\zeta$-potential values for different samples investigated during E2 sensing with the parent 75-mer and truncated aptamers.

\begin{tabular}{lll}
\hline \multicolumn{3}{c}{$\zeta$-potential $\mathrm{mV}(\mathrm{STD}, \mathrm{n}=3)$} \\
\hline Sample & No E2 & $1 \mu \mathrm{M} \mathrm{E2}$ \\
\hline Bare AuNPs & $-23.5(0.3)$ & $-24.0(0.7)$ \\
75-mer aptamer & $-40.2(0.9)$ & $-32.3(0.9)$ \\
75-mer random & $-46.2(0.7)$ & $-47(1)$ \\
35-mer aptamer & $-29.0(1)$ & $-25.0(0.2)$ \\
35-mer random & $-41.0(1)$ & $-41.7(0.3)$ \\
22-mer aptamer & $-29.0(1)$ & $-24.3(0.8)$ \\
22-mer random & $-42.0(2)$ & $-38.0(0.7)$ \\
\hline
\end{tabular}

When the AuNP-35-mer and AuNP-22-mer aptamers were incubated with 1000 $\mathrm{nM} \mathrm{E2}$, the $\zeta$-potential increased to $-25 \mathrm{mV}$ and $-24 \mathrm{mV}$. These values are close to the original value of the bare AuNPs $(-23.5 \mathrm{mV})$, in contrast to the large residual difference observed when the same measurement was applied to the AuNP-75mer system above.

Tolerance to salt for the 35-mer and 22-mer aptamer-coated AuNPs was substantially reduced upon specific interaction with E2 (Fig. 3.6a and Fig. 3.7a), although not to the extent of bare AuNPs, suggesting that the residual interaction between target-bound aptamers and AuNPs is not entirely eliminated in the shorter aptamers. Again, randomised 35-mer and 22-mer sequences did not display any further changes in $\zeta$-potential (Table 3.1) or salt resistance after incubation with E2 (Fig. 3.6a and Fig. 3.7a), which confirms the specificity of the observation.

Titration of the AuNP-35-mer aptamer against E2 with the optimized salt concentration $(23.8 \mathrm{mM})$, Fig. 3.6p revealed a 25 -fold enhancement of level of detection compared with the 75-mer system, which is greater than expected from the modest enhancement in $K_{\mathrm{D}} \mathrm{s}$. E2 was detected down to $200 \mathrm{pM}$, and a good linear response $\left(\mathrm{R}^{2}=0.94\right)$ was observed between $200 \mathrm{pM}$ and $800 \mathrm{pM}$, beyond which E2 can easily be detected by naked-eye (top panel). 

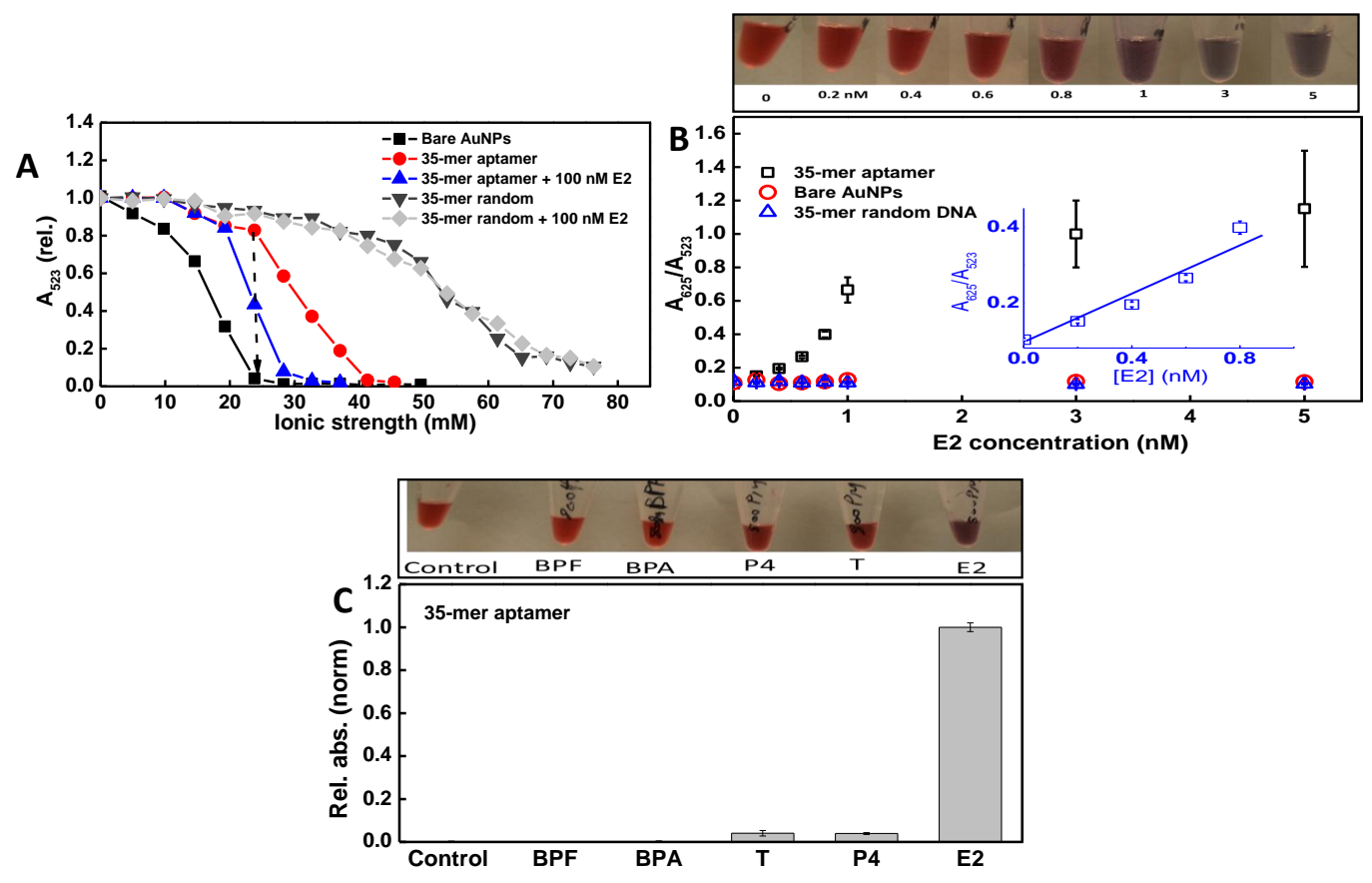

Figure 3.6: a) Salt dependent aggregation of AuNPs, AuNP-35-mer aptamer and AuNP-35-mer aptamer + $100 \mathrm{nM}$ E2 (the same type of data for the 35-mer random DNA is also shown), leading to determination of the optimal salt concentration for sensing (indicated by the black arrow). Aggregation is measured via the relative absorption at $523 \mathrm{~nm}$. b) Colorimetric sensing of a range of E2 concentrations using the AuNP-35-mer aptamer compared with bare AuNPs and AuNP-35-mer random DNA controls. c) Specificity examinations of interfering molecules (at $800 \mathrm{pM}$ ) using the AuNP-35-mer aptamer colloidal solution. Normalised absorptions at $523 \mathrm{~nm}$ were subtracted from the control and plotted as relative to E2 response. Error bars indicate standard deviation of the mean of three experiments. Full UV-vis spectra are provided in Fig. B.1-B.2.

Incubation of E2 with bare AuNPs and with a random 35-mer DNA coated AuNPs did not show any interaction (Fig. 3.6p). Fig. 3.6. shows the specificity examination of potentially interfering molecules at a concentration of $800 \mathrm{pM}$, where E2 is visually detectable (top panel). The 35-mer aptamer exhibited no nonspecific interaction with non-steroidal molecules, BPA and BPF, and in contrast to the 75-mer system, suppressed interference from T (4\%) and P4 (4\%).

Fig. 3.7p shows that the 22-mer system delivers the same detection limit as the 35 -mer (200 pM), although the magnitude of absorption change is approximately half the size for the 35-mer. As with the 35-mer, the enhanced detection limit towards E2 was also coupled to excellent discrimination against the entire suite of potentially interfering molecules that were screened (Fig. 3.7k). Incubation 
of E2 with bare AuNPs and with a random 22-mer DNA coated AuNPs did not show any interaction.
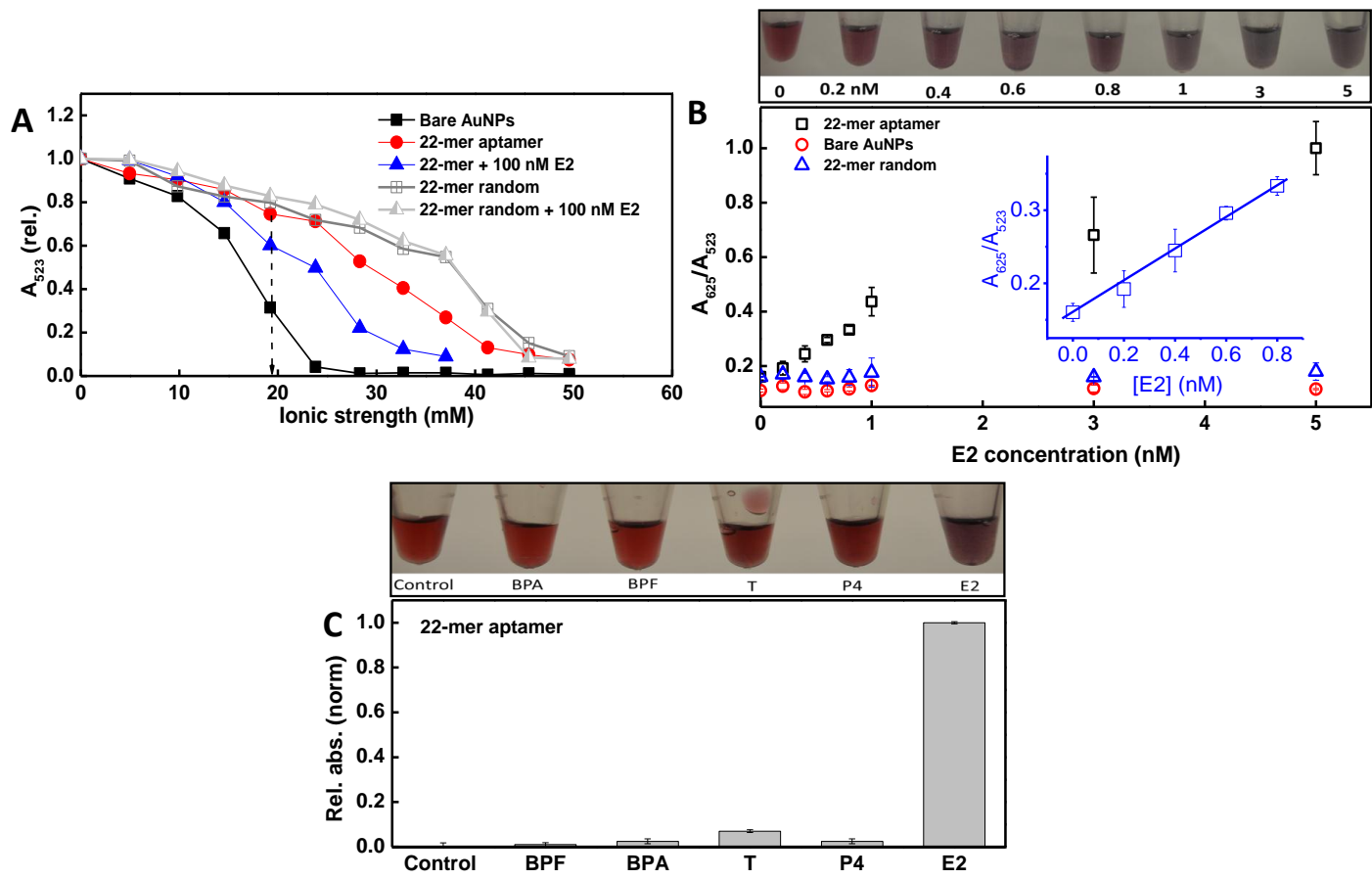

Figure 3.7: a) Salt dependent aggregation of AuNPs, AuNP-22-mer aptamer and AuNP-22-mer aptamer + $100 \mathrm{nM}$ E2 (the same type of data for the 22-mer random DNA is also shown), leading to determination of the optimal salt concentration for sensing (indicated by the black arrow). Aggregation is measured via the relative absorption at $523 \mathrm{~nm}$. b) Colorimetric sensing of a range of E2 concentrations using the AuNP-22-mer aptamer compared with bare AuNPs and AuNP-22-mer random DNA controls. c) Specificity examinations of interfering molecules (at $800 \mathrm{pM}$ ) using the AuNP-22-mer aptamer colloidal solution. Normalised absorptions at $523 \mathrm{~nm}$ were subtracted from the control and plotted as relative to E2 response. Error bars indicate standard deviation of the mean of three experiments. Full UV-vis spectra are provided in Fig. B.1-B.2.

\section{Electrochemical characterisation of aptamer surface dissociation}

To further investigate the surface interactions between AuNPs and each of the aptamers (with and without E2), chronocoulometry (CC) on AuNP electrodes was used to determine the surface densities of the aptamers before and after interaction with E2. This method is discussed briefly in this chapter. However, detailed background of CC measurements, AuNP electrodes formation, characterisations and experimental details are discussed in depth in Chapter 4.2 . 
Using the CC method developed by Steel et al, 133 surface-bound DNA was exposed to a positively charged redox probe (RuHex: $\mathrm{Ru}\left(\mathrm{NH}_{3}\right)_{6}{ }^{3+}$ ) that electrostatically binds to the phosphate groups. The DNA surface coverage density is related to the density of bound probes, which is measured via CC and separated from the unbound probe concentration via resolving time-dependent currents. AuNPs were electro-deposited on a glassy carbon electrode to form a dense layer of AuNPs and exposed to concentrated solutions of the 75-mer, 35-mer, or 22-mer aptamers. CC measurements obtained prior to and after exposure to the RuHex probe leading to measured surface coverage densities of $1.12 \times 10^{13}, 4.42 \times 10^{13}$, and $4.2 \times 10^{13}$ molecule $\mathrm{cm}^{-2}$ for the 75-mer, 35-mer, and 22-mer, respectively (Fig.3.8 and Table 3.2). The higher surface densities measured for the shorter aptamers may reflect their smaller surface requirements than the 75-mer.

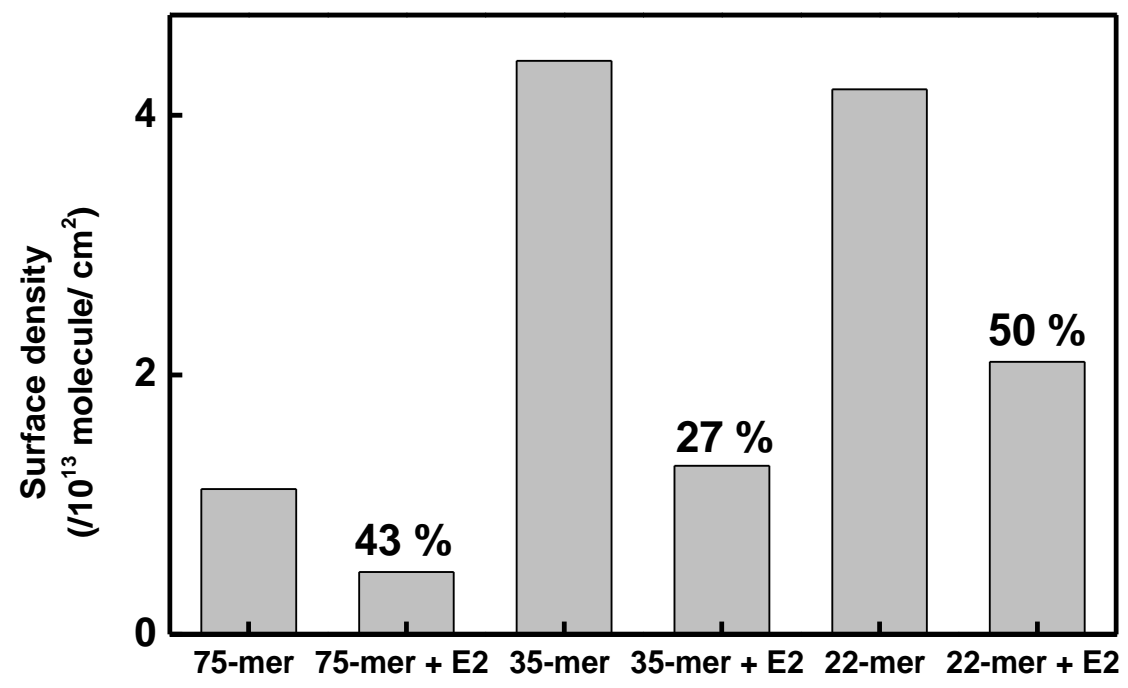

Figure 3.8: Surface densities of 75-mer aptamer, 35-mer aptamer, and 22-mer aptamer before and after incubation with $20 \mu \mathrm{M}$ E2. Further details regarding random sequence controls are found in Table 3.2 .

After incubation with $20 \mu \mathrm{M} \mathrm{E2,43 \%} \mathrm{of} \mathrm{the} \mathrm{75-mer} \mathrm{remained} \mathrm{bound} \mathrm{to} \mathrm{the} \mathrm{sur-}$ face. A more pronounced reduction in aptamer coverage was observed for the 35mer, with only $27 \%$ remaining adsorbed to the AuNP surface after E2 incubation. Consistent with the $\zeta$-potential and salt titration experiments mentioned earlier, the shorter sequence resulted in improved, although still incomplete, dissociation of target-bound aptamers from AuNP surfaces. Although the residual fractions of adsorbed aptamers differed by less than a factor of two, this can account for the substantially different extent of aggregation near the stability threshold that colorimetric sensors operate. This provides further support for the proposed 
hypothesis that excess bases suppress signal transduction and explains much of the sensitivity enhancement observed for the 35-mer over the 75-mer, in addition to the slightly enhanced $K_{\mathrm{D}}$ value. Control experiments using randomized sequences verify the specificity of aptamer dissociation (Table 3.2).

Table 3.2: Surface densities for different samples investigated during E2 sensing with the parent 75-mer and truncated aptamers.

\begin{tabular}{llc}
\hline \multicolumn{2}{c}{ Surface density $/ 10^{13}$ molecule $/ \mathrm{cm}^{2}$} \\
\hline Sample & No E2 & $20 \mu \mathrm{M} \mathrm{E2}$ \\
\hline Bare AuNPs & & - \\
75-mer aptamer & 1.12 & 0.48 \\
75-mer random & 1.01 & 1.04 \\
35-mer aptamer & 4.42 & 1.3 \\
35-mer random & 4.08 & 3.4 \\
22-mer aptamer & 4.2 & 2.1 \\
22-mer random & 4.9 & 4.6 \\
\hline
\end{tabular}

The 22-mer did not produce further improvement in aptamer dissociation from AuNPs, in spite of the more aggressive elimination of bases outside of the putative target-binding domain. This observation is in line with the smaller amplitude colorimetric sensing response (Fig. 3.7p) compared with the 35-mer identified in Fig. 3.6p. It appears that retaining a small number of bases outside of the putative binding region is optimal. This unexpected result highlights the fine balance that must be achieved between the aptamer's interaction with the AuNPs and with the target. By design, the 22-mer contains no redundancy outside of the likely target-binding region, meaning that bases committed to target binding cannot easily bind to AuNPs. However, corollary is that for 22-mer to adsorb to the AuNPs in the first place, they may remain trapped in structures that do not interact with the target. Target binding aptamer structures accessible in solution will not contribute to the sensor if they are not first adsorbed to AuNPs. On the other hand, the 35-mer had enough additional bases to assist the weak adsorption of structures presenting a favourable target-binding domain, but not so many that target-bound aptamers do not subsequently dissociate. 


\section{Sensing E2 in spiked urine samples with the 75-mer and 35-mer aptamers}

Finally, having demonstrated a route to enhanced sensitivity and selectivity towards E2, the operation of E2 sensor in the more challenging matrix of rat urine was examined. The colorimetric AuNP sensor format has yet to be implemented in urine, to the best of our knowledge. The challenge is illustrated by the significant aggregation observed when citrate coated AuNPs are exposed to urine (5\% by volume in water) without additional salt (top panel of Fig. 3.9a). The ionic strength of the rat urine $(2.1 \mathrm{mM}$, as estimated by the conductivity measurement shown in Fig. B.5, Appendix B is too low to account for the aggregation based on the stability of AuNPs up to $10 \mathrm{mM}$ ionic strength in water (Fig. 3.3 $)$ ). It is possible that specific complexation between adsorbed citrate species and specific metal ions including $\mathrm{Zn}^{2+}, \mathrm{Cu}^{2+}$, and $\mathrm{Fe}^{2+}$ may promote aggregation. 134135

Inclusion of the 35-mer aptamer prevents aggregation in the presence of $5 \%$ urine (by volume), and indeed leads to tolerance towards ionic strengths of over $60 \mathrm{mM}$ (over two-fold greater than in water, Fig. 3.9a vs. Fig. 3.3a). This observation further highlights the potential for interference from the urine matrix, for example the capacity of urine-based proteins and other molecules to sequester ions or adsorb on the surface of AuNP-aptamer (i.e. urine-based proteins and other molecules interact with the surface of aptamer-coated AuNPs to further stabilize them towards salt). Repeating the salt titration experiment in the presence of spiked E2 (Fig. 3.9 a) reveals an optimal ionic strength of $57 \mathrm{mM}$ for sensing E2 in rat urine (no reduction in salt resistance was observed for the random 35-mer sequence - Fig. 3.9a). Fig. 3.9p shows that spiked E2 was detected, visually and spectroscopically, from $5 \mathrm{nM}$ up to $1 \mu \mathrm{M}$, with a linear response from $50 \mathrm{nM}$ to $800 \mathrm{nM}\left(\mathrm{R}^{2}=0.95\right)$ before saturation occurs.

Fig. 3.9 also confirms that the sensor retains excellent discrimination against potentially interfering molecules in urine, including those from the same steroidal hormone family (additional details are provided in Appendix B in Fig. B.6- Fig. B.8). While the detection limit is 25 -fold lower than in water (Fig. 3.6a), the improvements resulting from removing flanking nucleotide sequences are still enough to detect biologically relevant concentrations of E2 in a complex physiological medium. ${ }^{136}$ On the other hand, the level of detection was found to increase by three orders of magnitude to $5 \mu \mathrm{M}$ for the longer 75-mer system (Fig. 3.9p, and UV-vis spectra in Fig. B.8 Appendix B), which would prevent practical application of the 75-mer. 

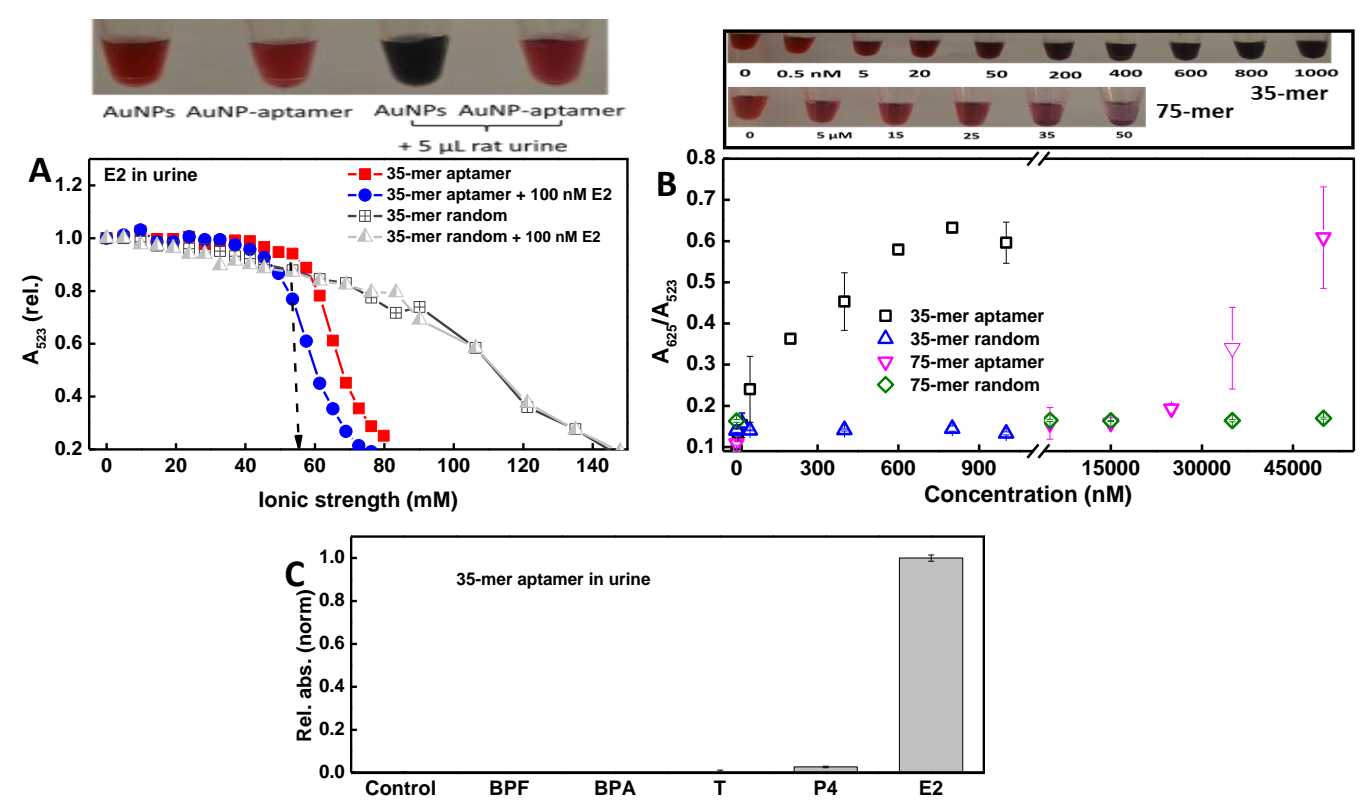

Figure 3.9: a) Salt dependent aggregation of AuNP-35-mer aptamer and AuNP-35mer aptamer + $100 \mathrm{nM}$ E2 in rat urine (the same type of data for the 35-mer random DNA is also shown), leading to determination of the optimal salt concentration for sensing (indicated by the black arrow). Aggregation is measured via the relative absorption at $523 \mathrm{~nm}$. Photographs of AuNPs and AuNP-35-mer aptamer before and after addition of $5 \mu \mathrm{L}$ rat urine are shown. b) Colorimetric sensing of E2 concentrations in spiked rat urine using the AuNP-35-mer and AuNP-75-mer aptamers compared with AuNP-35-mer and 75-mer random DNAs control. c) Specificity examinations of interfering molecules (at $200 \mathrm{nM}$ ) in rat urine samples using the AuNP-35-mer aptamer colloidal solution. Normalised absorptions at $523 \mathrm{~nm}$ were subtracted from the control and plotted as relative to E2 response. Error bars indicate standard deviation of the mean of three experiments.

\subsection{Chapter conclusion}

The chapter presented the development of a simple and reliable colorimetric aptasensor for the detection of E2, with a detection limit of $200 \mathrm{pM}$ and with discrimination against molecules belonging to the same steroidal family as E2. Starting from the 75-mer aptamer, the sequence was truncated to generate 35- and 22-base variants. The resulting performance of the 35-mer aptamer surpasses existing colorimetric aptasensors and the improvement was attributed to two factors; i) slightly improved aptamer affinity for E2 when the flanking nucleotide sequences were eliminated, and ii) suppressed residual affinity of target-bound aptamers to 
AuNPs, which was proven via $\zeta$-potential measurements and chronocoulometry. In spite of the minimalistic 22-mer also being effective, close comparison of its AuNP surface interactions vs. those of the 35-mer showed that a small number of excess bases are required in order to achieve the optimal balance of affinity to AuNPs and the target. Finally, it was demonstrated that the benefit of eliminating redundant bases from the aptamer sequences used in AuNP assays is amplified in biologically complex samples, enabling the first demonstration of a colorimetric AuNP sensor in urine. The present work provides a highly effective sensor scheme for sub-nanomolar E2 detection, as well as a simple methodology that is broadly applicable to the growing number of colorimetric aptasensors. More generally, this work demonstrates the benefit of editing aptamer sequences with specific regard to the way signals are transduced in a given aptasensor platform.

\subsection{Experimental section}

Chemicals and Materials used in this Chapter can be found in Appendix $\mathrm{D}$.

\section{Synthesis of AuNPs}

AuNPs (10 nm in diameter, TEM images in Fig. 3.2 were synthesized by the reduction of $\mathrm{HAuCl}_{4}$ with sodium citrate. ${ }^{137}$ Briefly, an aqueous solution of $\mathrm{HAuCl}_{4}$ $(100 \mathrm{~mL}, 1 \mathrm{mM})$ was stirred vigorously at $250{ }^{\circ} \mathrm{C}$ and a solution of sodium citrate $(10 \mathrm{~mL}, 38.8 \mathrm{mM})$ was added immediately. After boiling for $10 \mathrm{~min}$, the solution was stirred for another $15 \mathrm{~min}$ at room temperature and then stored at $4{ }^{\circ} \mathrm{C}$ for later use. The concentration of AuNPs was estimated to be $14 \mathrm{nM}$ from the calculation based on Beer-Lambert law using an extinction coefficient of $2.710^{8} \mathrm{M}^{-1}$ $\mathrm{cm}^{-1}$ at $525 \mathrm{~nm} .138$

\section{Formation of AuNP-aptamer hybrids}

Before exposure to aptamers, AuNPs were purified in order to minimise the citrate stabilisation and make their colloidal stability more sensitive to the presence of aptamers on their surfaces. Purification was achieved via 1:10 dilution of AuNPs in deionized water (Milli-Q, $18.2 \mathrm{M} \Omega \mathrm{cm}$ ), centrifugation at 12,500 rpm for 15 min (MIKRO 120- Hettich) and then resuspension to the initial concentration in 
deionized water (Milli-Q, 18.2 M $\Omega \mathrm{cm})$. Removal of excess citrate was confirmed by $\zeta$-potential values of $(-33.46 \mathrm{mV}( \pm 0.35))$ before and $(-23.5 \mathrm{mV}( \pm 0.28))$ after purification. $3 \mathrm{~mL}$ of the purified AuNPs was immediately mixed with $0.1 \mathrm{nmol}$ of the different aptamers, 75-mer, 35-mer, and 22-mer (from a solution of aptamer in deionized water (Milli-Q, 18.2 M $\mathrm{cm}$ )) (or random DNA, in the case of control experiments) to yield an aptamer concentration of $33.3 \mathrm{nM}$ and an aptamer: particle ratio of 3:1, for a particle number of $2.5 \times 10^{13}$. The suppressed target sensitivity observed for higher aptamer loading is presented in Fig. 3.1. NP-aptamer samples were prepared 1 hour prior to the sensing experiments. The sequences of different DNAs are provided in Appendix $\mathrm{D}$.

\section{Salt titration}

Salt titration experiments: The ionic strength was independently adjusted by adding different volumes of $0.5 \mathrm{M} \mathrm{NaCl}$ to $100 \mu \mathrm{L}$ samples of bare AuNPs, AuNPaptamer or AuNP-aptamer + 100 nM E2 (the same experiments were repeated with the respective random DNA polymers. The samples were left for $15 \mathrm{~min}$ and UV-vis absorption was measured, using the relative absorption at $523 \mathrm{~nm}$ $\left(\mathrm{A}_{523}\right.$ (rel.)) to evaluate the degree of aggregation.

\section{Target detection}

Target detection: Water was collected from the Hutt River, Wellington, New Zealand and pre-treated via stirring $50 \mathrm{~mL}$ overnight at room temperature with $1 \mathrm{~g}$ of activated charcoal to eliminate any traces of organic compounds, including estrogenic compounds, and filtering twice with $0.22 \mu \mathrm{m}$ syringe-filters. The conductivity was $100 \mu \mathrm{s} \mathrm{cm}^{-1}$ (at $25^{\circ} \mathrm{C}$ ), indicating very low salt content, and the $\mathrm{pH}$ was 8 . It was verified that the sensor exhibited the same behaviour when using deionized water (Milli-Q, $18.2 \mathrm{M} \Omega \mathrm{cm}$ ) instead of river water. Stock solutions of the targets were made in ethanol before adding appropriate volumes to the water and adjusting the final ethanol content to 5\%, ensuring sufficient target solubility. 20 $\mu \mathrm{L}$ of the resulting test samples were added to $100 \mu \mathrm{L}$ of AuNP-aptamer solution to obtain different E2 concentrations in a total reaction volume of $120 \mu \mathrm{L}$. Control samples comprised blank water containing 5\% ethanol. Samples were then incubated for $10 \mathrm{~min}$ at room temperature to facilitate binding to the target. The optimized $\mathrm{NaCl}$ concentration to trigger aggregation $(10 \mathrm{mM}$ for bare AuNPs, $23.8 \mathrm{mM}$ 75-mer and 35-mer aptamers and $19.8 \mathrm{mM}$ for the 22-mer aptamer), 
followed by gentle shaking, visual inspection after $15 \mathrm{~min}$ and measurement of UV-vis absorption of $5 \mu \mathrm{L}$ aliquots using a Thermo Scientific NanoDrop ${ }^{T M} 1000$ Spectrophotometer.

Rat urine was collected; by Dr. Christine Stockum, from the School of Biological Sciences, Victoria University of Wellington; from sexually mature ship rats (Rattus rattus). Then it was filtered with $0.22 \mu \mathrm{m}$ syringe-filters and spiked with E2 and interfering molecules after adjusting the content of ethanol to $5 \%$ (control rat urine sample comprised blank rat urine containing $5 \%$ ethanol). $5 \mu \mathrm{L}$ of spiked urine was added to $100 \mu \mathrm{L}$, AuNP-75-mer aptamer, AuNP-35-mer aptamer, or AuNP-22-mer aptamer (and the respective random DNA sequences), incubated at $50{ }^{\circ} \mathrm{C}$ for $10 \mathrm{~min}$, followed by addition of optimised $\mathrm{NaCl}(57.4 \mathrm{mM})$, gentle shaking, visual inspection after $15 \mathrm{~min}$ and measurement of UV-vis absorption as previously described.

\section{Sensitivity examination experiments for the 75-mer Aptamer}

Sensing of E2 with various concentrations of the 75-mer aptamer were done as previously described but with different salt concentration. The concentrations of $\mathrm{NaCl}$ for each system were as follow 23.8, 28.3 and $45.4 \mathrm{mM} \mathrm{NaCL}$ for 33.34, 66.67 , and $100 \mathrm{nM} 75$-mer aptamer respectively.

\section{Transmission electron microscopy (TEM) and dynamic light scattering (DLS) size characterisation}

TEM was performed on a JEOL 2010 microscope $(200 \mathrm{kV}) .5 \mu \mathrm{L}$ of AuNP samples was cast onto carbon-coated copper grid and followed by evaporation of the solvent under vacuum. A Zetasizer Nano ZS (forward scattering mode, Malvern Instruments, UK) with a $633 \mathrm{~nm}$ red laser was used for the size measurements. Salt was added to the $120 \mu \mathrm{L}$ samples, which were then diluted to $1 \mathrm{~mL}$ using deionized water (Milli-Q, $18.2 \mathrm{M} \Omega \mathrm{cm}$ ), placed in a $1 \mathrm{~cm}$ path length disposable cuvette, inserted in the Zetasizer and equilibrated at $25^{\circ} \mathrm{C}$ for 2 minutes prior to data acquisition. 


\section{$\zeta$-potential}

$\zeta$-potential measurements: $120 \mu \mathrm{L}$ samples of AuNPs alone, AuNP-aptamer and AuNP-aptamer + 1000 nM E2 (previously incubated at room temperature for 1 hour) without salt added were centrifuged at 12,500 rpm for $15 \mathrm{~min}$ (i.e, to remove excess aptamer) and then re-suspended in $1 \mathrm{~mL}$ using deionized water (Milli-Q, $18.2 \mathrm{M} \Omega \mathrm{cm}$ ) (the same experiments were done with the respective random DNA controls). Samples were loaded into a folded capillary cell, which was inserted into a Zetasizer Nano ZS equipped with a $633 \mathrm{~nm}$ laser (Malvern Instruments, UK) and equilibrated at $25^{\circ} \mathrm{C}$ for $2 \mathrm{~min}$ prior to undertaking $\zeta$-potential measurements in triplicate (reported as average value \pm standard deviation as shown in Table 3.1. The following parameters were fixed: $\mathrm{pH} 7$; viscosity 0.887 $\mathrm{mPa}$ s; and refractive index 1.33).

\section{DNA surface density measurements via chronocoulometry (CC)}

After deposition of AuNPs on glassy carbon electrode as detailed and characterised in Chapter 4, $0.1 \mathrm{nmol}(16.7 \mu \mathrm{M})$ of 75-mer aptamer, 35-mer aptamer and 22-mer were incubated with AuNP electrode for 20 min (a control experiment via exposing the aptamers on GCE resulted in no adsorption). $20 \mu \mathrm{M}$ of E2 was made in BWB as described previously, Chapter 2, and incubated with AuNP electrode coated with aptamers for $15 \mathrm{~min}$. The same experiments were repeated with the respective random DNA sequences as control experiments. The DNA surface density on AuNP electrode (before and after sensing of E2) was determined using the CC method developed by Steel et al. $\frac{133}{13}$ The adsorbed DNAs (aptamers or random DNAs) on AuNP electrodes were first immersed in a low ionic strength electrolyte, $20 \mathrm{mM}$ tris- $\mathrm{HCl}$ buffer (2-amino-2-(hydroxymethyl) propane-1,3-diol hydrochloride) at a $\mathrm{pH} 7.4$, the potential stepped from 200 to $-500 \mathrm{mV}$ versus (Ag/ $\mathrm{AgCl})$ for $500 \mathrm{~ms}$ (using BAS 100A electrochemical analyser), and the resulting charge flow was measured. The electrode was then immersed for $20 \mathrm{~min}$ in a solution of $150 \mu \mathrm{M} \mathrm{Ru}\left(\mathrm{NH}_{3}\right)_{6}{ }^{3+}$ (RuHex) $10 \mathrm{mM}$ tris-HCl buffer at a $\mathrm{pH} 7.4$, and the measurement repeated. Details of extracting the aptamer densities are discussed in depth in the next Chapter 4 in addition to determination of the surface area of AuNP electrode. 
The procedure for estimating the $K_{\mathrm{D}}$ of the 35-mer and 22-mer was based on that used for the 75-mer previously described in Chapter 2. As shown in Fig. B.9, the measured fluorescence intensities at $310 \mathrm{~nm}$ were first converted to concentration via an E2 calibration curve (with detection limit of $5 \mathrm{nM}$ ). By expressing a measured E2 concentration as an unbound fraction, $1 / f_{a}$, as a function of $1 /$ total E2 concentration, the $K_{\mathrm{D}}$ was determined as $14 \pm 2 \mathrm{nM}$ and $11 \pm 1 \mathrm{nM}$ for 35-mer and 22-mer, respectively, and applying Eq. 2.5, refer to Fig. 3.5. A saturation offset at $\mathrm{f}_{\mathrm{a}}=0.6$ was observed due to partial loss of the NP-35-aptamer and NP-22-mer aptamer conjugates during the centrifugation, and renormalized to $f_{a}(\max )=1$. Excitation and emission slit widths were set to the relatively low resolution of 5 $\mathrm{nm}$ and $10 \mathrm{~nm}$ respectively in order to enhance the measured fluorescence intensities in such dilute samples. 


\section{Chapter 4}

\section{Electrochemical impedance spectroscopy based aptasensors}

\subsection{Abstract}

This chapter describes two different electrochemical impedance spectroscopy (EIS) based aptasensors for E2. The first approach affords a femtomolar detection limit of the target and excellent specificity against interfering compounds. This sensor features the E2 aptamers functionalized via amide bonds to a conducting polymer modified electrode whose surface potential is probed via EIS. E2 binding causes a redistribution of negative charges in the electrode double-layer region when the aptamer adopts a folded conformation around the small neutral target molecule.

As an alternative strategy, the non-specific adsorption of aptamers onto Au surfaces is investigated and contrasted with the conventional tethering approach using thiol-gold bonds. Using EIS and cyclic voltammetry (CV), the interfacial properties of flat and nanoparticulate gold electrodes coated with aptamers via both binding modes are found to be profoundly different. While tethered aptamers suppress interfacial charge transfer from a redox probe, adsorbed aptamers are found to markedly enhance charge transfer, which is explained via the redistribution and displacement of surface ions. Moreover, the non-specifically adsorbed aptamer completely coats the surface to passivate it against spurious interactions, as well as cross-linking the nanoparticulate electrode. This combination of properties results in a simply fabricated and highly effective sensor for E2, whereby increased charge-transfer resistance $\left(R_{C T}\right)$ is resolved when targetbound aptamers dissociate from the electrode surface. The sensor exhibited a 
femtomolar level of detection, excellent selectivity against potentially interfering molecules, and robust operation in urine samples. The simplicity and performance of the sensor platform renders it applicable to a wide range of other targets for which aptamer sequences are available.

\section{Structure of the chapter}

An overview of EIS is firstly presented followed by a brief discussion of the work carried out to construct an EIS based aptasensor for E2 using conduction polymer modified electrodes. In the second half of the chapter, a novel aptasensing scheme for E2 based on EIS and the non-specific adsorption of aptamers on $\mathrm{Au}$ surfaces is presented. Both aspects of this chapter were carried out as a collaboration with Bicheng (Amy) Zhu and Professor Jadranka Travas-Sejdic of the University of Auckland, collectively producing two manuscripts for publication. In this chapter, the scope of discussion of the conducting polymer related work (for which I am the second author on the corresponding paper) is limited to describing the basic properties and mechanism of the sensor. These features will then be contrasted with the Au based system (for which I am the lead author on the manuscript), which will be described in greater depth in this chapter. Further details of the conducting polymer sensor is published in the literature (accepted article, Biosensors and Bioelectronics, doi:10.1016/j. bios. 2015.03. 050) and will also be published in Amy Zhu's thesis.

\subsection{Overview of EIS}

Electrical resistance is the ability of a circuit element to resist the flow of electrical current described by Ohm's law, Eq. 4.1.

$$
R=\frac{V}{I}
$$

as the ratio of voltage (V) and current (I). The resistance is used for electrical circuits where it is independent of frequency and the alternating current (AC) and voltage signals through a resistor are in phase with each other.

However, real world electrochemical cells present much more complex behaviour where resistance and capacitance have to be considered. Electrical impedance accounts for the total opposition to the flow of an AC current at a given frequency. 
In an Electrochemical cell, electrical impedance $(Z)$ is typically measured by a technique known as electrochemical impedance spectroscopy (EIS). EIS measurement involves application of a constant bias plus small sinusoidally oscillation potential over a range of frequencies (modulated from high to low) and measurement of the current response. ${ }^{57}$ From this measurement it is apparent that the impedance is the ratio of the voltage-time function $V(t)$ and the resulting currenttime function $\mathrm{I}(\mathrm{t})$ :

$$
Z=\frac{V(t)}{I(t)}=Z_{r e}+j Z_{i m}
$$

where $\mathrm{j}$ is $\sqrt{-1}$. ${ }^{139}$ The $\mathrm{I}(\mathrm{t})$ can differ in terms of the amplitude and show a phase shift in comparison to the $V(t)$ function. Thus, the impedance value can be described by the real part of impedance $Z_{\mathrm{re}}$ and the imaginary part of impedance $\mathrm{Z}_{\mathrm{im}}$ as described by Eq. 4.2. The data is commonly presented by Nyquist plots as shown in Fig. 4.1a. The Randles circuit, shown in Fig. 4.1p, is used to separate different components present in EIS data. $\frac{5657}{5 I S}$ components includes charge transfer resistance $\mathrm{R}_{\mathrm{CT}}$ (inversely proportional to the rate of electron transfer) that presents at the kinetically controlled domain at high frequencies $(\omega)$, double layer capacitance $C_{d}$, solution resistance $R_{s}$, and Warburg impedance $Z_{w}$ (arises from mass transfer limitations at low $\omega$ ).

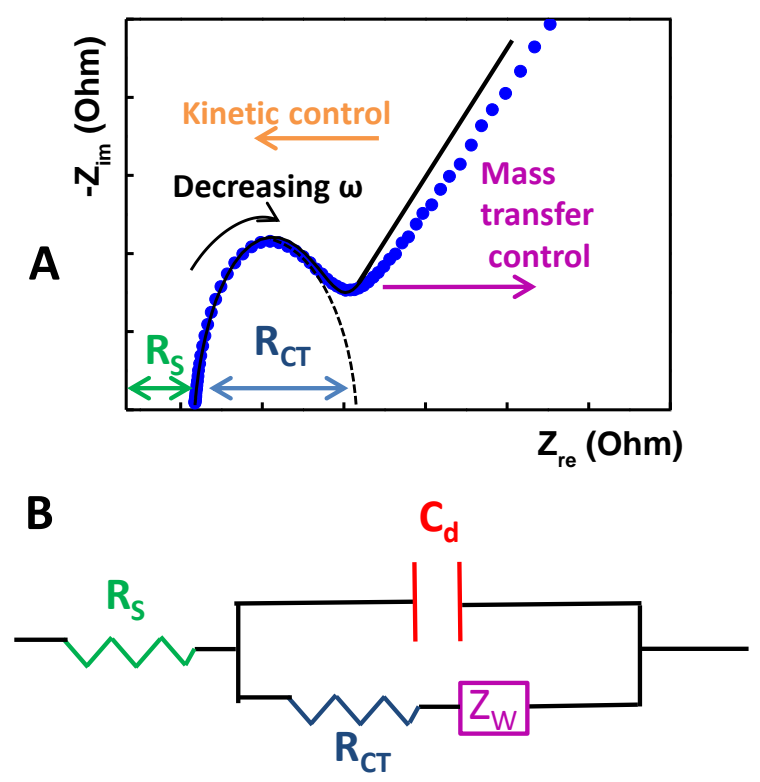

Figure 4.1: a) An example of EIS signal output represented as Nyquist plot, with the key processes and parameters highlighted. Data points (symbols) are fitted with b) Randles equivalent circuites of resistors and capacitors to interpret the electrochemical system. 
A freely diffusing redox reporter, typically ferro/ferricyanide couple $\left[\mathrm{Fe}(\mathrm{CN})_{6}\right]^{-3 /-4}$, is used in EIS to probe the surface modification. Changing the diffusional access of the redox reporter to the electrode surface impacts the rate of redox conversion and hence attenuation in EIS is seen as an increase or decrease of the charge transfer resistance $\mathrm{R}_{\mathrm{CT}}$. ${ }^{[56,139}$

EIS is particularly well-suited for the detection of binding events on the electrode surface as well as characterising surface modifications. The method has found increasing application in the so-called label-free DNA sensing using various electrode surfaces including gold ${ }^{140 \mid 141}$ and conducting polymers. ${ }^{142+144}$ Covalent coupling of the polyionic ssDNAs to the surfaces via amide bond (OC-NH) or Au-S bond results in self-assembled monolayers that increase the electrode surface potential near the electrical double layer which act as a kinetic barrier limiting the redox conversion reaction and transduced as an increase in $\mathrm{R}_{\mathrm{CT}}$. $\frac{142[145 \mid 146}{1}$ Hybridising a target DNA to form dsDNA alters the double layer potential and/or potential distribution and consequently change in the kinetics of the redox reaction which determines $\mathrm{R}_{\mathrm{CT}}$.

\subsection{E2 aptasensing based on conducting polymer}

\subsubsection{Introduction}

The following section will briefly discuss the first application of conducting polymer based electrodes in small molecule sensing, particularly the detection of E2. Previous research from our collaborator, Professor Jadranka Travas-Sejdic (School of Chemical Sciences, University of Auckland), indicated a remarkable success of conducting polymer based electrodes in DNA sensing. $142+144 \mid 147148$ The previously demonstrated DNA sensors show that electrochemically conducting polymers (ECPs) are ideal for probing charge redistribution within a few nanometers of the electrode surface, they are easily grown via electropolymerisation and covalently functionalized with DNA, their redox properties are well-matched to ferricyanide probes, and their nanoporous morphology amplifies effects within the electric double-layer region. Therefore, an ECP (poly-pyrrole-pyrroleacrylic acid) was selected to construct a sensor for E2 as well as to understand the signal transduction mechanisms for neutral low MW targets. 


\subsubsection{Results and discussion}

\section{Construction of electrochemically conducting polymer electrode}

Fig. 4.2 shows different steps were undertaken to construct the aptasensor. The nanoporous ECP electrode was grown by electropolymerisation of pyrrole (Py) and 3-pyrroleacrylic acid (PAA), with a mole ratio of Py:PAA 25:1, on a glassy carbon electrode (GCE) to yield a copolymer, poly(Py-co-PAA) following previous reports ${ }^{143 \mid 149}$ (SEM image is shown in Fig. 4.2p). Subsequently, the COOH groups were activated by EDC/NHS to mediate an amide bond formation with the amine terminated 75-mer aptamer, as previously described in Chapter 2 with carboxylated polystyrene NPs.

EIS is an ideal tool to probe step by step surface modifications as shown in Fig. 4.2. The change in the interfacial surface properties of the aptamer functionalised ECP electrode in a phosphate-buffered saline solution was recorded in presence of the anionic redox probe $\left[\mathrm{Fe}(\mathrm{CN})_{6}\right]^{-3 /-4}$. The initial reduction in $\mathrm{R}_{\mathrm{CT}}$ indicated by size of the semicircular component in the low $\mathrm{Z}$ region, is characteristic of high surface area ECP film formation. ${ }^{143}$ The covalent tethering of the negatively charged DNA aptamer increases $\mathrm{R}_{\mathrm{CT}}$ owing to the electrostatic barrier impeding access of the anionic redox probe. ${ }^{143] 146}$ Accordingly, it was verified the covalent nature of aptamer attachment by observing a lack of $\mathrm{R}_{\mathrm{CT}}$ response when the coupling reagents (EDC/NHS) were omitted. Further treatment of the surface with $5 \mathrm{nM}$ of $\mathrm{E} 2$ resulted in a pronounced increase in $\mathrm{R}_{\mathrm{CT}}$ induced by binding the target.

\section{E2 sensing with the 75-mer aptamer tethered to ECP electrode}

Having established a hybridised ECP electrode with the 75-mer aptamer with an initial promising signal for $5 \mathrm{nM} \mathrm{E2}$, the sensitivity and specificity of the sensor was investigated. Fig. 4.3 a shows the sensor response, presented as Nyquist plots, towards E2 over a wide concentration range when the same electrode was sequentially exposed to higher E2 concentrations in BWB. Fig. 4.3b shows the same data presented in differential form $\left(\Delta \mathrm{R}_{\mathrm{CT}} / \mathrm{R}_{\mathrm{CT}}{ }^{0}\right)$ to quantify the normalized $\mathrm{R}_{\mathrm{CT}}$ variation compared with the aptamer-functionalized electrode without exposure to E2. The pronounced monotonic increase in $\Delta \mathrm{R}_{\mathrm{CT}} / \mathrm{R}_{\mathrm{CT}}{ }^{0}$ can be attributed to specific aptamer-target binding by observing a negligible response towards E2 when the aptamer is omitted (base line response seen in Fig. 4.3 b). 


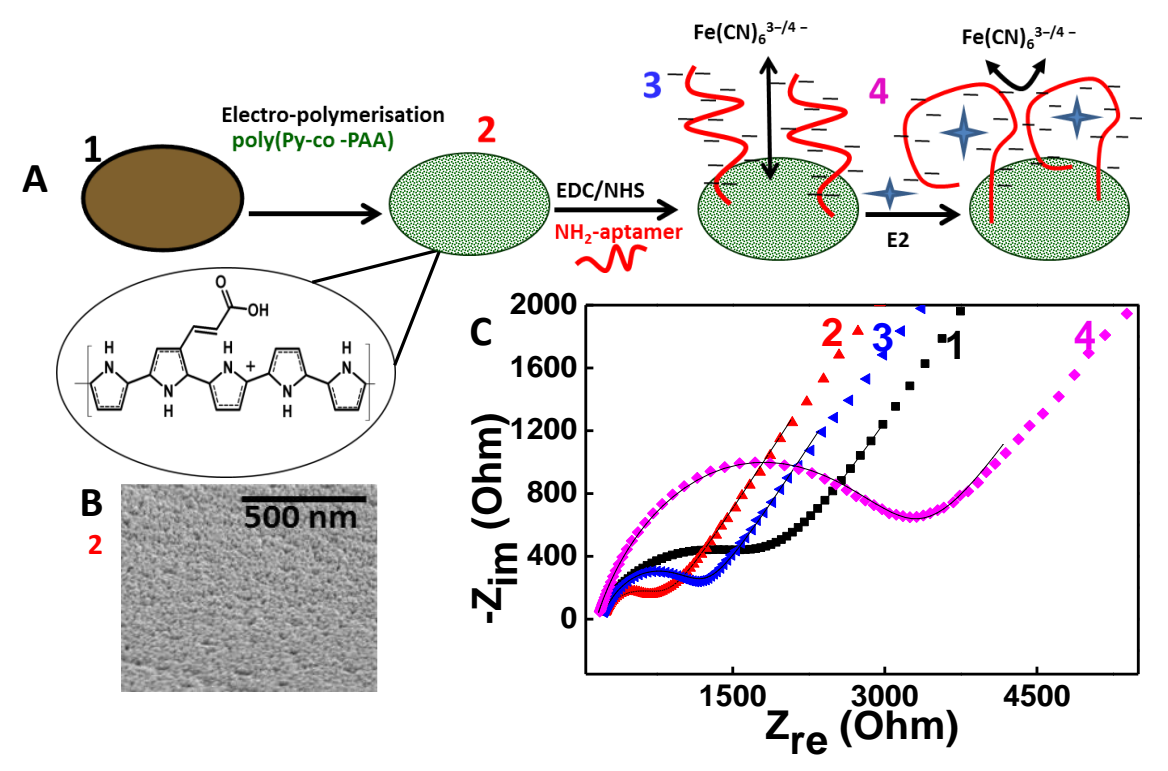

Figure 4.2: a) Construction of the aptasensor and schematic representation of E2 sensing. b) SEM image for electropolymerised poly(Py-co-PAA) film. c) EIS spectra, presented as Nyquist plots of different electrodes (1) bare GCE, (2) after electropolymerisation, (3) after immobilization of the 75-mer aptamer, and (4) after incubation with $5 \mathrm{nM}$ E2. Experimental data (symbols) are fitted to the Randles equivalent circuit model.

Remarkably, E2 was detected as low as $1 \mathrm{fM}$, with a monotonic quasi-logarithmic response over 9 orders of magnitude up to $1 \mu \mathrm{M}$. Error bars shown in Fig. 4.3b are standard deviation of the mean of three individual experiments conducted with three different electrodes. The strongly amplified $\mathrm{R}_{\mathrm{CT}}$ signals are explained as a result of conformational changes of the aptamer within the double layer upon binding the low molecular weight target E2. Binding-induced redistribution of aptamer charges towards the electrode surface can account for the observed increased $\mathrm{R}_{\mathrm{CT}}$ which indicates a slower rate of redox conversion and limited surface access of the $\left[\mathrm{Fe}(\mathrm{CN})_{6}\right]^{-3 /-4}$ (as depicted in $4.2 \mathrm{a}$ ). The EIS results are consistent with the previously noted compact conformation with the size based aptasenor, Chapter 2, when the 75-mer aptamer recognised its target. 

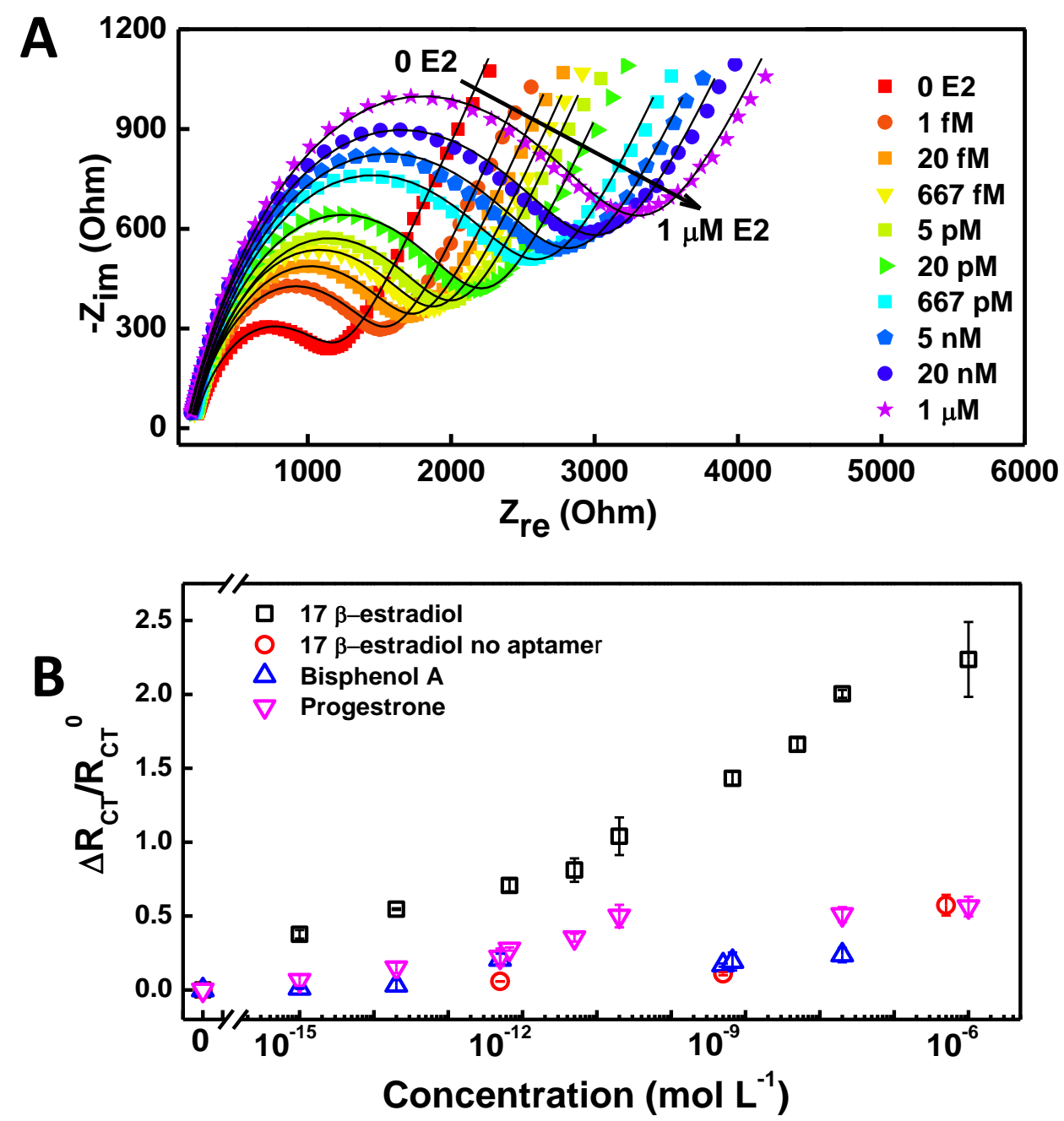

Figure 4.3: a) EIS spectra presented as Nyquist plots characterising the response towards E2. Experimental data (symbols) are fitted to the Randles cell equivalent circuit (lines). b) Differential change in $\mathrm{R}_{\mathrm{CT}}$ vs. concentration of E2, progesterone, and bisphenol-A. The response towards E2 is also shown for a control electrode lacking the aptamer (when the aptamer was exposed to the surface with no activation using EDC/NHS). Error bars represent standard deviations from three individual experiments.

Specificity of the 75-mer aptamer: A comparison with pervious aptasensors in this thesis

The sensor exhibited excellent discrimination against a structurally similar steroidal hormone progesterone (P4) and a non-steroidal estrogenic compound bisphenolA (BPA). It should be noted that the 75-mer aptamer showed affinity towards P4 when sensing with TRPS (Fig. 2.9) and with the colorimetric sensor (Fig. 3.3). 
However, EIS based sensor showed excellent discrimination against P4. The differences in the non-specific interaction observed with aptamer using the various sensors developed in this thesis could be attributed to the experimental set up of the sensors. Sensing with EIS involves exposing the aptamer functionalized electrode to a target for a given amount of time, followed by thorough washing steps with BWB to remove unbound target molecules, and then EIS measurements are taken. It could be speculated that, initially, the 75-mer aptamers could weakly bind P4 molecules, but subsequent washing steps could cause the molecules to be removed. On the other hand, in solution sensing of P4 molecules, by TRPS and the colorimetric aptasensors, is not subjected to any washing steps where weakly associated molecules could be removed.

Sensing with the 75-mer aptamer versus sensing with the 35-mer aptamer

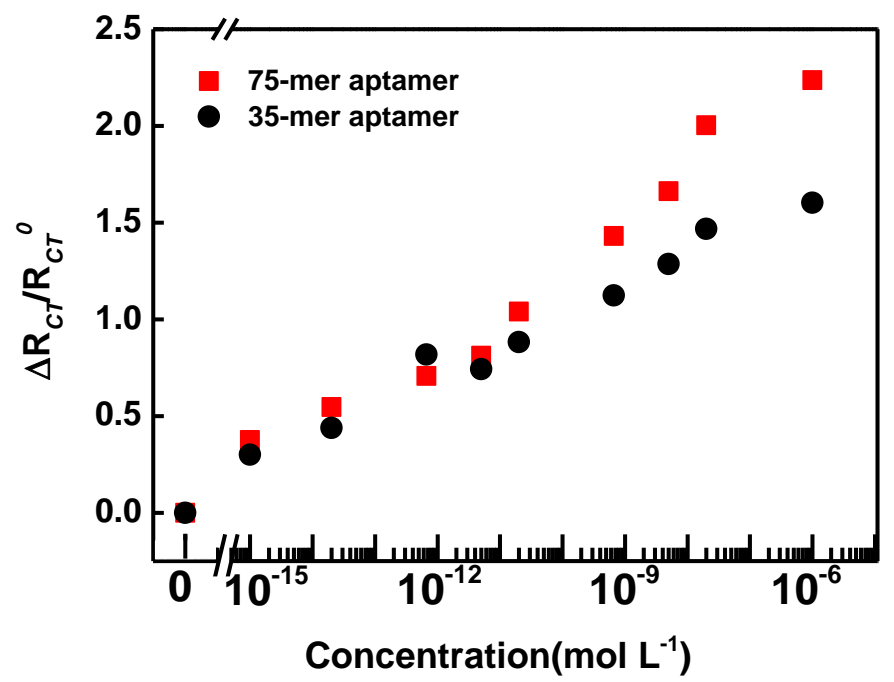

Figure 4.4: Differential change in $\mathrm{R}_{\mathrm{CT}}$ vs. concentration of $\mathrm{E} 2$, comparing the response of the 75-mer aptamer against the response of the 35-mer aptamer.

Close comparison between the performance of the 75-mer and 35-mer aptamers tethered to ECP electrode revealed that the excess flanking nucleotides could play a favourable role by enhancing $R_{\mathrm{CT}}$ signal when bringing more charges to the double layer region. In contrast, a weaker response was observed with the 35mer after $1 \mathrm{nM}$ concentration of E2, as shown in Fig. 4.4. In the colorimetric aptasensor, the 35-mer showed a 25-fold increase in level of detection of E2 in comparison to the 75-mer aptamer. In fact, it is the way aptamer's signal is transduced by the aptasensor platform that determines the performance of long or truncated sequences. 


\subsection{EIS-based E2 sensing with non-specifically adsorbed aptamers on Au electrodes}

\subsubsection{Introduction}

Monolayer films of ssDNA probes, including aptamers, are extensively functionalised onto Au electrodes for diagnostic and bioanalytical applications. In DNAbased electrochemical sensors, the DNA is mostly modified with a thiol group at one end to facilitate a strong covalent tether to the Au electrode and allow the DNA probe molecule to extend away from the surface. $\frac{150 \cdot 152}{15}$ When the polyanionic DNA recognises its target to form double stranded DNA ${ }^{142145}$ or a DNA aptamer-target complex, ${ }^{69.153}$ charges are redistributed in the electric double layer region. Small interfacial charge redistribution substantially alters the redox kinetics of extrinsic molecules, $\left[\mathrm{Fe}(\mathrm{CN})_{6}\right]^{-3 /-4}$, which is commonly quantified via changes in the $\mathrm{R}_{\mathrm{CT}}$ using EIS.142[145]146 This is overall what is seen in the conducting polymer case (Section 4.3), but with amide coupling.

After functionalising Au electrodes with thiolated DNA when fabricating bioanalytical devices, it is essential to back-fill the remaining exposed Au surface with short alkylthiol chains in order to eliminate spurious signals from non-specific adsorption to the electrode. ${ }^{154[155}$ The maximum packing density of tethered DNA is approximately $5 \times 10^{13} / \mathrm{cm}^{2}, 156$ which is constrained by the steric requirements of free DNA strands that leaves vacant thiol binding sites being on the underlying surface. ${ }^{157}$ Besides the numerous unknown constituents of the test solution, DNA itself can also adsorb to exposed Au surfaces via its nitrogen bases. 158 .159 Non-specific DNA adsorption is perceived to be undesirable in electrochemical sensors using DNA probes, however, it has yet to be explored as an alternative strategy in itself, in spite of several potential advantages.

One motivation for pursuing electrochemical DNA aptasensors that have nonspecifically adsorbed DNA probes is that perturbations associated with target binding must occur in direct proximity to the Au electrode surface. In an electrolyte with 1.7 M ionic strength (typical for EIS of biological systems), 142]143]149 the Debye screening length is less than $1 \mathrm{~nm}$, and a large fraction of the electrode potential is compensated by the very first layer of ions. Secondly, by exploiting weak adsorption interactions that demand extensive surface contact, it was hypthesized that the requirement for additional surface passivation steps might be eliminated when Au electrodes are saturated with DNA probes covering the 
surface. Thirdly, as discussed in Chapter 3, the operation of colorimetric aptasensors demonstrates that DNA molecules adsorbed onto Au surfaces are still able to recognise target molecules. In colorimetric sensors, the adsorbed DNA stabilizes Au nanoparticle dispersion towards salt-induced aggregation. The DNA probes can dissociate from the nanoparticle surface upon interaction with the target, which triggers aggregation and thus a visible colour change. This concept has been successfully applied to the detection of specific DNA sequences, small molecules, 115$] 126,127$ and proteins, ${ }^{45}$ frequently achieving nanomolar to subnanomolar levels of detection. $\underline{38}$

In this research, the interfacial properties of non-specifically adsorbed DNAs on flat and nanoparticulate Au electrodes compared with those covalently tethered via thiol linkers were investigated, as depicted in Scheme 4.1. It was demonstrated that the surface orientation of the DNA strongly affects the interfacial charge distribution, leading to pronounced differences resolved by EIS and cyclic voltammetry. In comparison to thiol tethered DNA, surface adsorbed DNA leads to decreased $R_{C T}$ values, lower total DNA content under saturation, as well as effective passivation, and stabilization of $\mathrm{Au}$ nanoparticle (AuNP) electrodes. These properties were all explained via the different binding modes and they were exploited in a new class of electrochemical aptasensor that was demonstrated in this chapter. The investigation focused on the 35-mer aptamer for E2 that was found to be effective in the colorimetric aptasensor, as discussed in Chapter 3. This simple new sensor has a $1 \mathrm{fM}$ level of detection, wide dynamic range (comparable to the EIS based ECP sensor for E2 using the 75-mer aptamer), excellent selectivity against potential interfering molecules, and robust operation in urine - even in the absence of passivation steps.

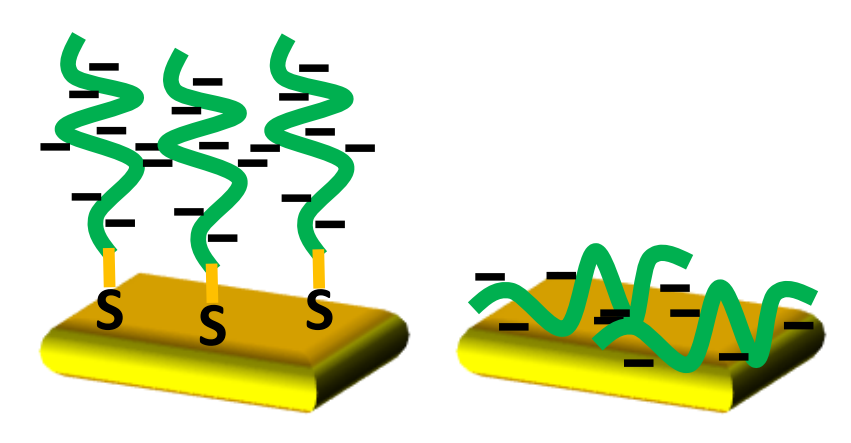

Scheme 4.1: Illustration of different coupling modes of thiolated aptamers (covalent) and non-thiolated aptamers (non-specific adsorption). 


\subsubsection{Results and discussion}

\section{Covalent tethering versus non-specific adsorption: The effect on interfacial ion distribution}

Fig. 4.5 shows EIS data presented as Nyquist plots for a flat Au electrode functionalised with a thiolated 35-mer DNA aptamer for E2 compared with the same aptamer lacking the thiol group, as well as the bare electrode. The composition of the coupling solution and the coupling protocol was identical for the thiolated and non-thiolated DNA samples, other than the presence of the thiol group, as detailed in the Experimental Section of this Chapter. The Nyquist plots are fitted to the Randles cell equivalent circuit, from which the interfacial $R_{C T}$ is extracted as the diameter of the characteristic semicircle. Fig. 4.5 shows that covalently coupling the 35-mer DNA aptamer increased the $\mathrm{R}_{\mathrm{CT}}$ from $\sim 332 \Omega$ to $1700 \Omega$, consistent with previous EIS investigations employing covalently tethered DNA. ${ }^{146 / 161+163}$ The observed $\mathrm{R}_{\mathrm{CT}}$ increase is explained by the negative charge of the DNA impeding the access of negatively charged $\left[\mathrm{Fe}(\mathrm{CN})_{6}\right]^{-3 /-4}$ redox probe molecules near the surface. However, the non-specifically adsorbed 35-mer DNA aptamer results in significantly reduced $\mathrm{R}_{\mathrm{CT}}$, from $\sim 332 \Omega$ to $32 \Omega$.

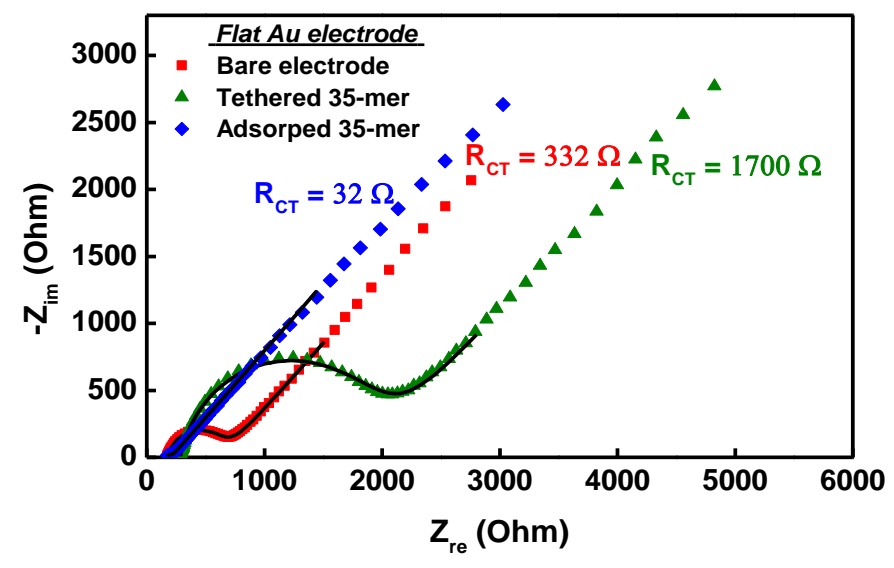

Figure 4.5: EIS spectra, presented as Nyquist plots for a flat Au electrode modified with the 35-mer aptamer via covalent and non-specific couplings. The Randles equivalent circuit model was used to fit the experimental data (symbols) of EIS.

To understand this effect, it is necessary to consider the charge distribution near the electrode and how it is perturbed for both configurations of DNA attachment. When a metal electrode comes into contact with an electrolyte solution, an electric double layer is created by strongly polarised electrolyte ions at the charged 
electrode surface. ${ }^{164}$ The length scale of the double layer region can be estimated by the Debye screening length $\lambda_{\mathrm{D}}$ :

$$
\lambda_{D}=\sqrt{\frac{\epsilon_{0} \epsilon_{r} k_{B} T}{2 N_{A} e^{2} I}}
$$

where $\epsilon_{0}$ is the permittivity of free space, $\epsilon_{r}$ the dielectric constant of the electrolyte, $k_{\mathrm{B}}$ the Boltzmann's constant, $\mathrm{T}$ the temperature, $\mathrm{N}_{\mathrm{A}}$ the Avogadro's number, e the charge of an electron, and I the ionic strength. For the $1.7 \mathrm{M}$ ionic strength electrolyte solution used here (defined as $I=1 / 2 \sum C_{i} Z_{i}^{2}$ ), the Debye length is only $0.7 \mathrm{~nm}$. Due to this high ionic strength, and since the EIS measurement is conducted relatively high potential of $+0.23 \mathrm{~V}$, a significant fraction of the potential will be compensated by a very dense layer of chloride anions at the $\mathrm{Au}$ surface. $\frac{165}{16}$ The distribution of anions close to the electrode strongly affects the $\mathrm{R}_{\mathrm{CT}}$ experienced by the anionic $\left[\mathrm{Fe}(\mathrm{CN})_{6}\right]^{-3 /-4}$ probe approaching the surface.

It can be considered how the different binding modes of DNA will perturb the interfacial charge distribution. The intrinsic negative charge density of DNA can be approximated as $2.7 \times 10^{21}$ charges $/ \mathrm{cm}^{3}$ based on the structure of doublestranded DNA (assuming a cylinder with $2 \mathrm{~nm}$ diameter, $0.34 \mathrm{~nm}$ spacing, and two charges per base pair). The intrinsic charge density of DNA is significantly higher than in the bulk electrolyte beyond the double layer $\left(9 \times 10^{19}\right.$ charges $/ \mathrm{cm}^{3}$, based on the electrolyte concentration of $0.15 \mathrm{M}$ used here). The thiol tethered 35 -mer aptamer can extend up to $\sim 12 \mathrm{~nm}$ away from the surface, which is essentially bulk solution for such a short Debye length. Even in a more relaxed conformation, most of the aptamer's negative charges will be far enough from the surface (beyond the Debye length) that they increase the negative charge density, thereby impeding access of negatively charged redox species and increasing the measured $\mathrm{R}_{\mathrm{CT}}$ value.

On the other hand, adsorption to the surface via weak base interactions requires significant surface contact, meaning that a much higher fraction of the aptamer volume must lie in direct proximity to the electrode. In order to achieve sufficient contact with the Au surface, the DNA may displace more surface charge density than the intrinsic charge density that it brings to the surface, as depicted in Scheme 4.2. The concentration of counter-ions in the diffuse region of an electrode interface can be estimated by the Boltzmann equation:166

$$
c_{i}=c_{0} e^{\frac{-Z_{i} F \Psi}{k T}}
$$


Where $c_{i}$ and $c_{0}$ represent the ion concentration at the interface and bulk respectively, $\mathrm{z}$ is charge of the ion, $\Psi$ the electrostatic potential, $\mathrm{T}$ the temperature, $\mathrm{F}$ Faraday's constant, and k the Boltzmann constant. An interfacial charge density equivalent to that of the intrinsic DNA charge density $\left(\sim 2.7 \times 10^{21}\right.$ charges $\left./ \mathrm{cm}^{3}\right)$ corresponds to a potential difference of only $7.3 \mathrm{mV}$ in the diffuse interface region relative to the bulk for a $1.7 \mathrm{M}$ ionic strength solution. Considering the higher applied electrode potential $(230 \mathrm{mV})$, and the higher ion density in the Stern layer compared with the diffuse region, it was concluded that the anion density within the first nanometer of a bare electrode under EIS conditions must be substantially higher than the intrinsic charge density of DNA. Conversely, when DNA displaces surface charge to adsorb to the electrode, the net surface charge density is diluted by the DNA. The $\left[\mathrm{Fe}(\mathrm{CN})_{6}\right]^{-3 /-4}$ redox probe will thus experience a lower barrier to approaching the surface occupied by adsorbed DNA, which can account for the lower $\mathrm{R}_{\mathrm{CT}}$ value measured.

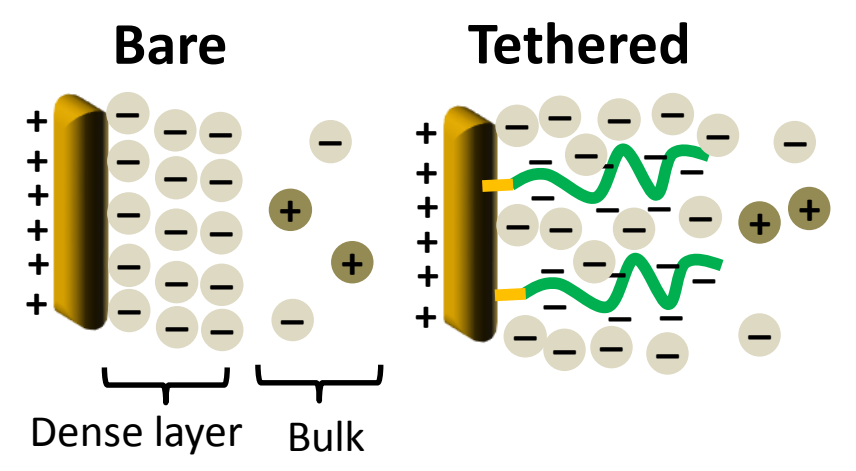

Adsorbed

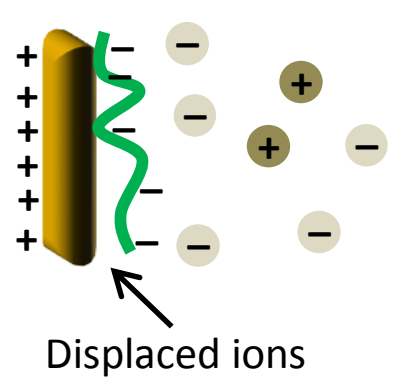

Scheme 4.2: Ion distribution around a bare Au electrode at high potential compared with electrodes with aptamers coupled via different modes.

\section{Generation of AuNP based electrodes}

AuNP electrodes offer the benefit of higher surface area, 167 as well as the ability to be grown onto any conductive substrate, including glassy carbon electrodes $(\mathrm{GCE})^{\sqrt{168}}$ and conducting polymers. ${ }^{169}$ Here, AuNPs were electrodeposited from a solution containing $1 \mathrm{mM} \mathrm{HAuCl}_{4}$ on a bare GCE surface. The roughened but uniform morphology of the AuNP based electrode is confirmed by the SEM images in Fig. 4.6a. The surface roughness can be obtained by applying the RandlesSevcik equation, Eq. 4.5.164]170/171

$$
i=2.68 \times 10^{5} n^{2 / 3} A D^{1 / 2} v^{1 / 2} c^{0}
$$

where $\mathrm{i}$ is peak current, A the electrode surface area, D the diffusion coefficient, $v$ the potential scan rate, and $c^{0}$ the mediator concentration. AuNP electrode was 
found to have a roughness factor of approximately 2 (and surface area of 0.015 $\mathrm{cm}^{2}$ vs. a known surface area $0.00785 \mathrm{~cm}^{2}$ of GCE) after applying Eq. 4.5 to scan-rate dependent cyclic voltammograms for the flat versus AuNP electrodes as shown in Fig. 4.6c. The increased surface roughness is in agreement with previous work. ${ }^{172}$ The calculations is detailed in Appendix C. Furthermore, Fig. 4.6p shows that modifying GCE with AuNPs leads to decreased $\mathrm{R}_{\mathrm{CT}}$ (from $\sim 4000$ $\Omega$ to $\sim 1000 \Omega$ ) on account of the increased surface area, which is also reflected in the increased peak anodic and cathodic currents measured via CV (Fig. 4.6d).
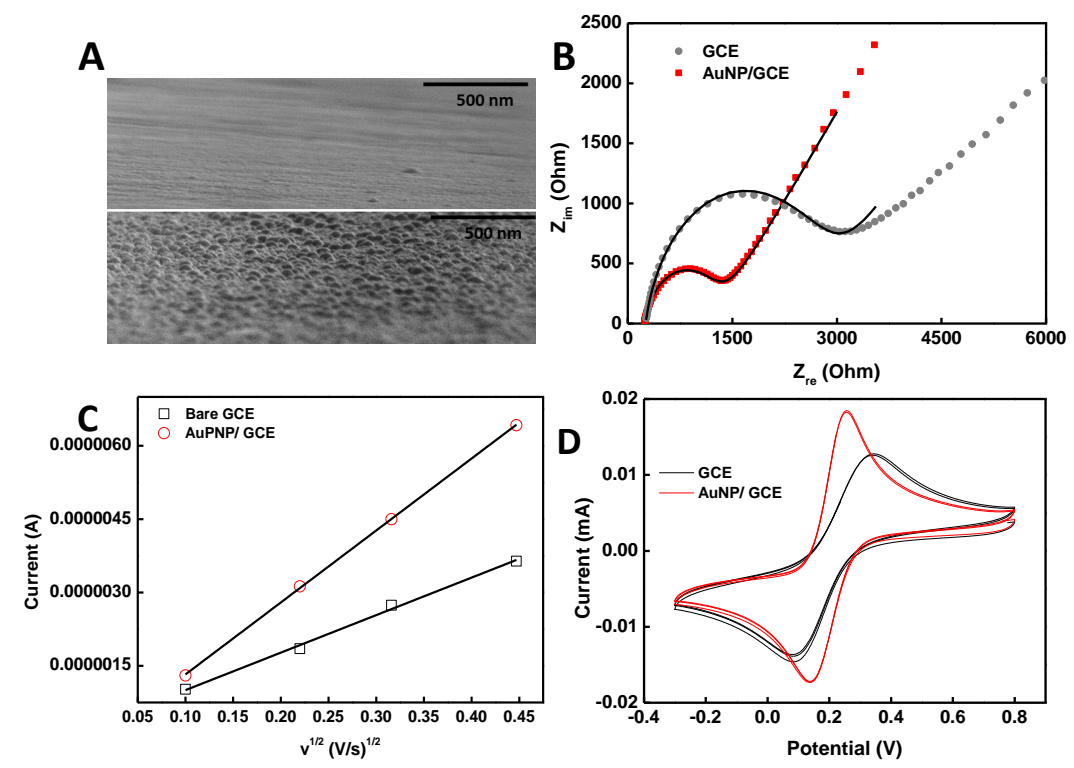

Figure 4.6: a) SEM image for glassy carbon electrode (top) and AuNP modified electrode (bottom). b) EIS spectra, presented as Nyquist plots for bare GCE electrode and after electrochemical deposition of AuNPs. c) Current vs. square root of scan rate for bare GCE and AuNP/GCE modified electrode for surface area calculation. d) Representative cyclic voltammetry for GCE and AuNP electrode at scan rate of $10 \mathrm{mV} / \mathrm{s}$.

\section{Covalent tethering versus non-specific adsorption with AuNP electrodes}

Fig. $4.7 \mathrm{a}$ shows the EIS response of the same pair of DNA aptamers functionalised on an electrode comprising electrodeposited AuNPs on GCE. When comparing the EIS response of thiol versus non-specific adsorption of DNA aptamers, essentially the same behaviour is observed on the AuNP as described above for the flat Au electrode (Fig. 4.5). The measured $\mathrm{R}_{\mathrm{CT}}$ of the bare AuNP surface $(\sim$ $1000 \Omega$ ) increased to $\sim 2500 \Omega$ for the thiol tethered aptamer, yet $R_{\mathrm{CT}}$ decreased to 
$190 \Omega$ for the non-specifically adsorbed aptamer. The same trends were observed when AuNPs were electrodeposited on a flat Au electrode (Fig. 4.8a).

Fig. 4.7b shows cyclic voltammograms for AuNP electrode compared with those functionalised with thiol-tethered and non-specifically adsorbed 35-mer aptamers. In each case, the pair of well-defined peaks at $\mathrm{E}^{0}=225 \mathrm{mV}$ vs SCE corresponds to the formal potential of the $\left[\mathrm{Fe}(\mathrm{CN})_{6}\right]^{-3 /-4}$ couple. The cyclic voltammograms of the three electrodes differ in their peak currents, which is related to the heterogeneous electron transfer rate. ${ }^{164}$ The CV results are consistent with the EIS data above, thiol tethered DNA leads to a suppressed interfacial electron transfer rate whereas the adsorbed DNA enhances the rate.
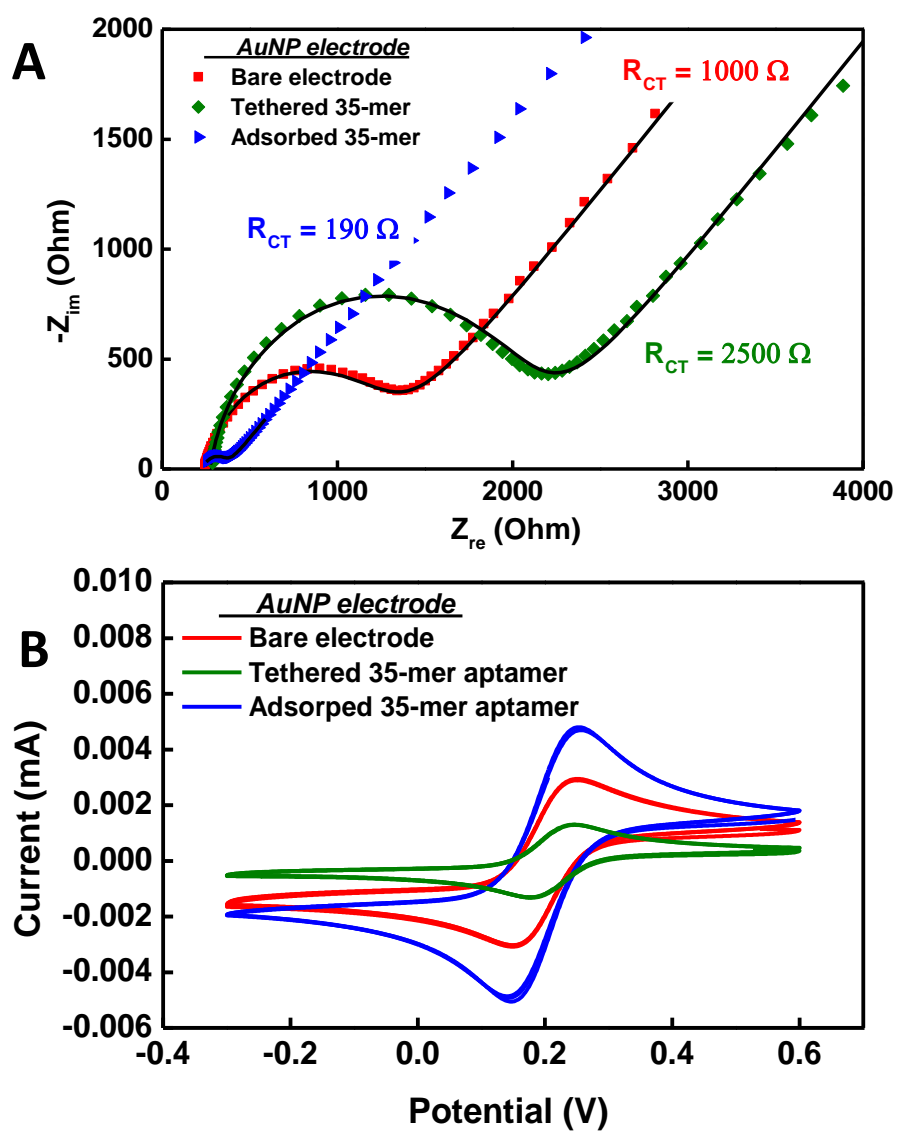

Figure 4.7: a) EIS spectra, presented as Nyquist plots for AuNP electrode modified with the 35-mer aptamer via covalent and non-specific couplings. The Randles equivalent circuit model was used to fit the experimental data (symbols) of EIS. b) Cyclic voltammetry conducted at a scan rate of $20 \mathrm{mV} / \mathrm{s}$ (in $5 \mathrm{~mL}$ PBS solution with $\left.5.0 \mathrm{mM}\left[\mathrm{Fe}(\mathrm{CN})_{6}\right]^{-3 /-4}\right)$ for AuNP electrode and covalent and non-specific couplings of the 35-mer aptamer.

It was confirmed that the observed decrease in $R_{C T}$ for adsorbed DNA was not 
confined to this particular 35-mer sequence, but a rather general phenomenon. As shown in Fig. 4.8, decreased $\mathrm{R}_{\mathrm{CT}}$ values were also observed to different degrees for non-specific adsorption of a 75-mer aptamer for E2 (covalent tethering is also shown for this sequence), randomised versions of the 35-mer and 75-mer, as well as 30-mer homonucleotides of poly-T, poly-C, and poly-G. Only poly-A caused the $\mathrm{R}_{\mathrm{CT}}$ value to increase slightly. In previous studies of 5-mer homooligonucleotides affinity to Au surfaces, poly-A was found to strongly dominate over the other bases and could compete effectively against thiol binding. ${ }^{173}$ Therefore, it is likely that the poly-A behaves more like a thiol tethered sequence, where a small segment of the DNA is in strong contact with the surface and the remaining strand dangles away from the surface
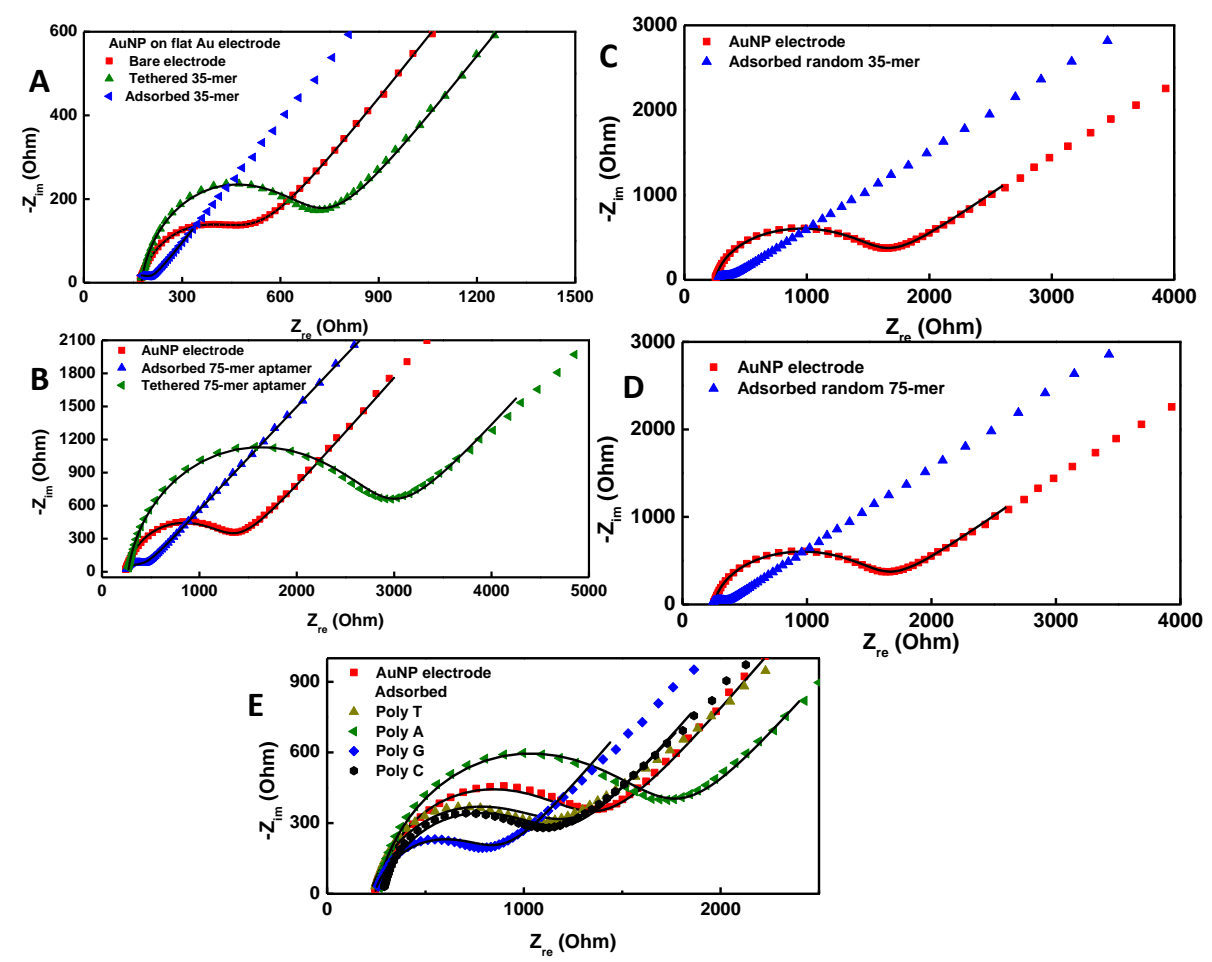

Figure 4.8: EIS spectra presented as Nyquist plots for a) Covalently tethered and non-specifically adsorbed 35-mer aptamer on a AuNP electrode deposited on flat $\mathrm{Au}, \mathrm{b})$ 75-mer covalently tethered and non-specifically adsorbed on a AuNP electrode grown on GCE, c) Random 35-mer non-specifically adsorbed on a AuNP electrode grown on GCE, d) Random 75-mer non-specifically adsorbed on a AuNP electrode grown on GCE, and e) 30-mer homonucleotides non-specifically adsorbed on a AuNP electrode grown on GCE. 


\section{Surface densities of the covalent tethering versus non-specific adsorption}

Having established that DNA binding mode strongly affects the interfacial charge transfer kinetics, the hypothesis that non-specific DNA adsorption could saturate the surface and eliminate the need for additional passivation steps was examined. The coupling densities of the DNA via each binding mode was determined using chronocoulometry. CC, as the name implies, involves measurement of the charge (coulombs) as a function of time (chrono) response to an applied potential step (instantaneous change) at the working electrode. The potential stepping takes place from a value where no reaction occurs to a value that leads to electrolysis (oxidation or reduction) of the electro-active species. CC is a powerful technique to characterised quantities of facially adsorbed electro-active material. Cottrell equation, Eq. 4.6, describes the relation between charge (Q) of the surface-confined redox species and the time $(t)$ in an electrochemical system: 164

$$
Q=\frac{2 n F A C_{0} D_{0}^{1 / 2}}{\pi^{1 / 2}} t^{1 / 2}+Q_{d i}+n F A \Gamma_{0}
$$

where $\mathrm{n}$ is the number of electrons, $\mathrm{F}$ the Faraday's constant, A the electrode surface area, $\mathrm{D}_{0}$ the diffusion coefficient, $\mathrm{C}_{0}$ the bulk concentration, $\mathrm{Q}_{d I}$ the capacitive charge, $\mathrm{nFA} \Gamma_{0}$ is the charge from the reduction of redox marker on the electrode, and $\Gamma_{0}$ represents the amount of redox marker on the electrode surface.

When $t=0$, the detected charge is the sum of $Q_{d l}$ and the surface excess terms, $\mathrm{nFA} \Gamma_{0}$. Capacitive charge $\left(\mathrm{Q}_{\mathrm{dl}}\right)$ and $\mathrm{nFA} \Gamma_{0}$ that quantifies the charge of the adsorbed electro-active species pass through very quickly in comparison to the slow diffusion component of the bulk and therefore can be separated in a plot of the total charge $Q$ vs. $t^{1 / 2}$. Hence, $Q_{d l}+n F A \Gamma_{0}$ values are obtained from the intercept. Surface excess $\left(\Gamma_{0}\right)$ of the adsorbed electro-active species is separated from $Q_{\mathrm{dl}}$ by performing an additional CC experiment in the supporting electrolyte that lacks the electro-active species $\frac{164}{16}$ and as illustrated for the tethered 35-mer in Fig. 4.9 .

Surface coupled DNAs can not be quantified by their own as they do not present quantifiable electro-activity. However, steel et al ${ }^{133}$ developed a DNA quantification method by exploiting the weak electrostatic interaction between DNA and a cationic redox marker $\mathrm{Ru}\left(\mathrm{NH}_{3}\right)_{6}{ }^{3+}$ (RuHex) in low ionic strength solutions. Redox cations exchange for native counterions associated with the DNA phosphate. The amount of redox marker electrostatically attached at the DNA is then determined using chronocoulometry as described before. The surface densities of the thiol tethered 35-mer aptamer and the adsorbed 35-mer were determined by us- 
ing the charges observed in Fig. 4.9 and applying Eq. 4.7.

$$
\Gamma_{D N A}=\Gamma_{0} \frac{Z}{m} N_{A}
$$

where $\Gamma_{\text {DNA }}$ is the DNA surface density (molecules $/ \mathrm{cm}^{2}$ ), $\mathrm{m}$ is the number of phosphate groups on the probe DNA, $\mathrm{z}$ is the charge on the redox molecule ( 3 for RuHex), and $N_{A}$ is Avogadro's number.

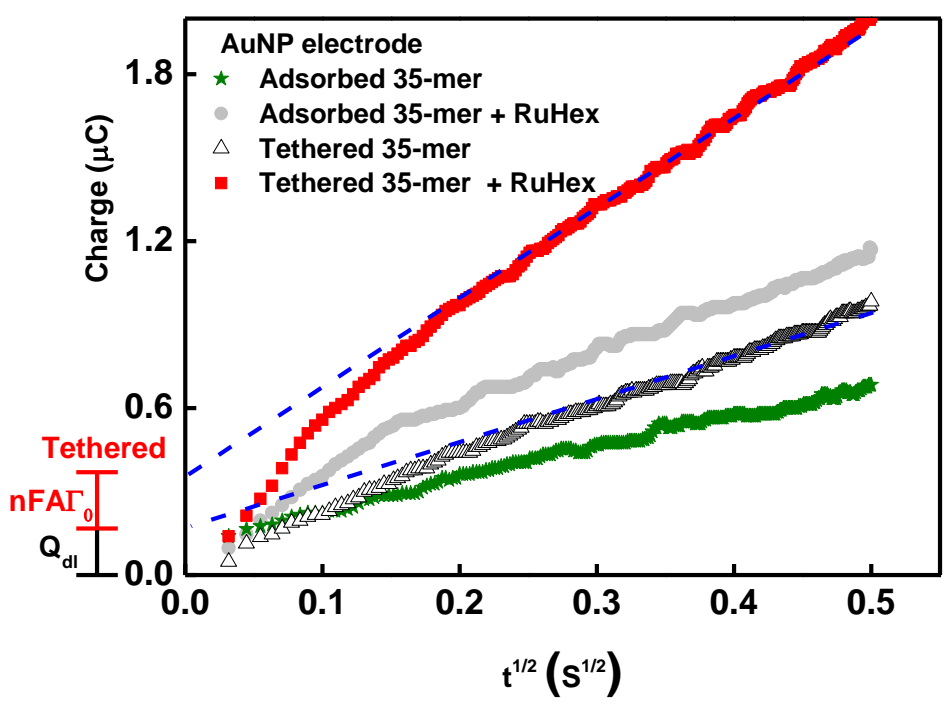

Figure 4.9: Chronocoulometry determination of the excess charge of the 35-mer aptamer coupled to the surface of AuNP electrode via covalent coupling and nonspecific adsorption. The dashed blue lines are fits for the covalently tethered 35mer to determine the charge intercept at $t=0$ before and after RuHex incubation and used to separate the term $n F A \Gamma_{0}$ from $Q_{\mathrm{dl}}$ as indicated in y axis. The fits of the adsorbed 35-mer results are not shown for clarity.

The tethered 35-mer aptamer had a surface density of $6.5 \times 10^{13}$ molecule $/ \mathrm{cm}^{2}$ or $0.64 \mathrm{~nm}^{2}$ per molecule, corresponding to the maximum steric close-packing of DNA. ${ }^{156}$ On the other hand, the surface adsorbed DNA had a surface density of only $4 \times 10^{13}$ molecule $/ \mathrm{cm}^{2}$, or $4 \mathrm{~nm}^{2}$ per molecule. The higher total density measured for thiol tethered DNA is consistent with previous measurements using X-ray photoelectron spectroscopy, ${ }^{155}$ fluorescence labelling, ${ }^{159}$ and chronocoulometry. $\frac{158}{15}$ This difference in coverage density is an expected consequence of DNA aligned along versus away from the surface, however, this measurement does not resolve whether any Au surface remains exposed in either case. 


\section{Examination of self-passivation characteristic of the adsorbed aptamer}

In order to probe whether each of the DNA attachment modes left accessible $\mathrm{Au}$ surfaces, they were exposed to the thiol molecule 6- mercaptohexanoic acid (MHA), as shown in Fig. 4.10a. The $\mathrm{R}_{\mathrm{CT}}$ substantially increases when a bare AuNP electrode is exposed to the negatively charged MHA, which like 6- mercaptohexanol is known to have a strong affinity for Au surfaces and forms a dense self-assembled monolayer. ${ }^{[158,159}$ When MHA is co-assembled with the thiol-tethered aptamer, a pronounced increase in $\mathrm{R}_{\mathrm{CT}}$ is resolved because even densely packed DNA leaves enough exposed Au for MHA to bind to the surface. On the other hand, MHA has no effect on the $\mathrm{R}_{\mathrm{CT}}$ of the non-specifically adsorbed DNA electrode. This is clear evidence that the aurophilic molecule MHA is blocked from accessing the surface, leading to the conclusion that non-specifically adsorbed DNA completely covers the Au surface. The surface was also exposed to bovine serum albumin (BSA) to represent the interactions found in a physiological medium. The $R_{C T}$ of a bare AuNP electrode is substantially increased upon exposure to BSA, however the invariance of $\mathrm{R}_{\mathrm{CT}}$ for the non-specifically adsorbed DNA electrode confirms that the surface is passivated against BSA adsorption.

Fig. $4.10 \mathrm{p}$ shows that the non-specifically adsorbed DNA aptamer plays an additional role in stabilising the AuNP electrodes. Electrodeposited AuNP electrodes are known to suffer from instability due to the loose electrostatic connectivity between the AuNPs, prompting others to cross-link them with dithiol molecules to $\mathrm{Au}$ electrodes $\frac{167}{167}$ or laborious specific derivatising of GCE with sulfhydrylterminated monolayer. $\frac{174}{174}$ The loss of AuNPs is clearly seen via increases in $R_{C T}$ (towards the flat GCE electrode value, Fig. C.1 in Appendix C) with sequential buffer washing steps (Fig. 4.10p).

The characteristic $R_{C T}$ increase was observed for both the bare AuNP electrode and the thiol-tethered DNA AuNP electrode, preventing its use as a sensor. However, the non-specifically adsorbed DNA suppresses this undesired drift in $R_{C T}$ after multiple cycles of washing with buffer. Repeating the measurement on a flat Au electrode (Fig. 4.10 F) confirms that the effect is attributed to the instability of the AuNP electrode. While previous sensors derived from AuNP electrodes required additional AuNP crosslinking steps as well as surface passivation steps, herein it was that the surface-adsorbed DNA probe fulfils both of these roles. 


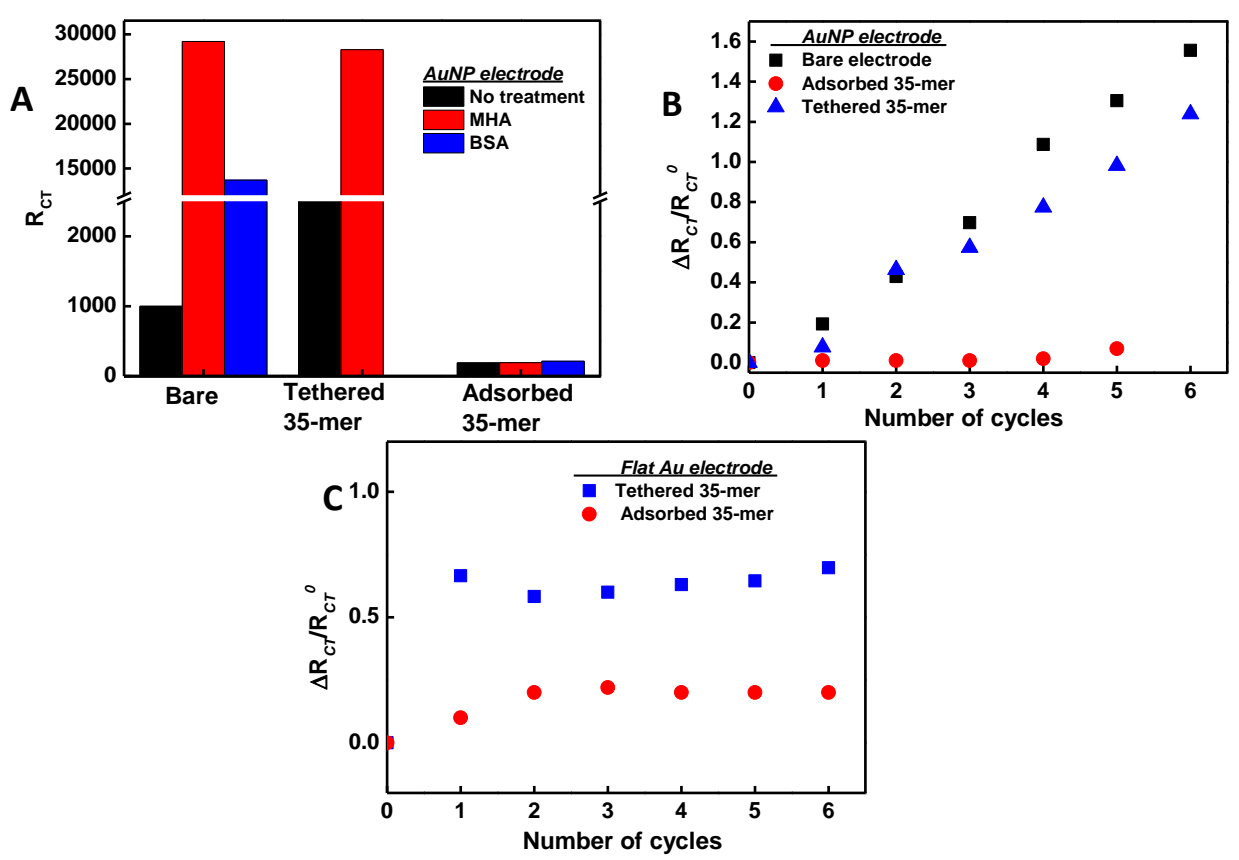

Figure 4.10: a) $R_{\mathrm{CT}}$ for AuNP electrode, covalently tethered 35-mer aptamer, and adsorbed 35-mer aptamer and treatment with MHA and BSA. b) Examination of AuNP loss after washing cycles with buffer. AuNP electrodes were either not coated, coated with the covalently tethered 35-mer aptamer, or non-specifically coated with the non-thiolated aptamer. c) Buffer washing cycles of a flat Au electrode modified with the covalently tethered 35-mer aptamer and the nonspecifically coated 35-mer aptamer.

Before examining whether the surface adsorbed DNA probes retain the ability to recognise their target, the initial conclusion can be drawn that non-specifically adsorbed DNA produces electrode interfaces with fundamentally different properties than thiol tethered DNA. In spite of a lower total DNA content, adsorbed DNA spreads along the Au surface with multiple base contact points. Important consequences of the observed difference in binding modes include; stronger perturbation of the charge distribution within the confined double layer region (and concomitantly reduced $\mathrm{R}_{\mathrm{CT}}$ values), passivation of the Au surface when it is saturated with DNA, and stabilisation of AuNP electrodes via cross-linking.

Sensing E2 with the covalently and adsorbed 35-mer aptamer on flat Au electrode

Since the AuNP-thiol-aptamer system could not be reliably used for E2 sensing due to the AuNP instability identified above, the response of thiolated aptamer 
to $\mathrm{E} 2$ on a flat $\mathrm{Au}$ electrode was initially examined. As previously discussed in Chapter 3, the 35-mer E2 aptamer has a $K_{\mathrm{D}}$ of $14 \mathrm{nM}$ and excellent selectivity against potential interfering molecules. Sequential E2 addition in buffer solution results in increased $R_{C \text { T }}$ values, as shown in Fig. 4.11(Nyquist plots are provided in Appendix C, Fig. C.2). This increase is consistent with the previously discussed EIS-based E2 sensor with conducting polymer and also consistent with a sensor for E2 employing thiol-tethered aptamer. ${ }^{68}$ The EIS signals can be understood as a result from an increased negative charge density when the target-bound aptamer adopts a more compact conformation. However, Fig. 4.11 also reveals the high background signal from E2 directly interacting with a bare Au surface, highlighting the need to backfill the electrode with a passivating agent. Accounting for the background signal leads to a level of E2 detection of approximately $20 \mathrm{nM}$ for the thiolated aptamer.

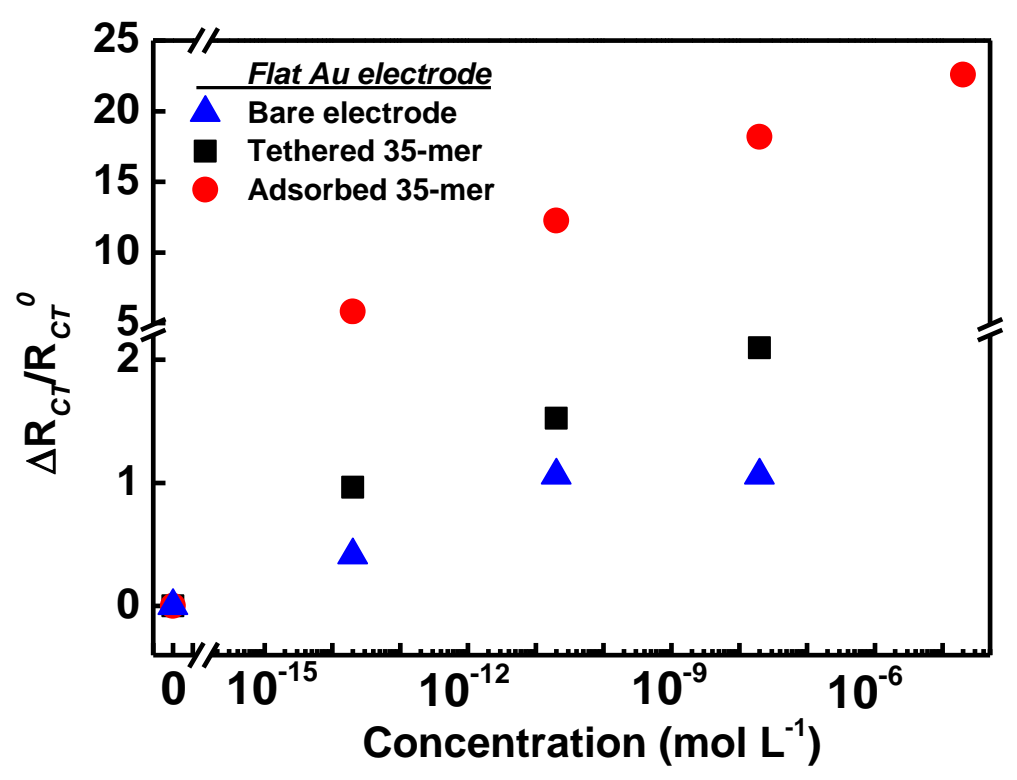

Figure 4.11: Differential change in $\mathrm{R}_{\mathrm{CT}}$ vs. concentration of $\mathrm{E} 2$ with the covalently tethered and non-specifically adsorbed 35-mer aptamer on a flat Au electrode, along with the response of the bare electrode.

Next, the response of the non-specifically adsorbed aptamer on flat Au electrode to E2 was examined. The precedence of the colorimetric AuNP sensor discussed in Chapter 3 suggests that the surface adsorbed aptamers may still recognise the E2 target, but the signal transduction mechanism is expected to be different than for the covalently tethered aptamer. In the colorimetric AuNP sensor, aggregation is triggered when target-bound aptamers dissociate from AuNP surfaces. Translating this behaviour to the present EIS sensor suggests that target binding should be associated with a signal concomitant with reduced DNA surface coverage. Since adsorption of DNA substantially reduced the $\mathrm{R}_{\mathrm{CT}}$, target binding 
should therefore result in increased $\mathrm{R}_{\mathrm{CT}}$ values. Fig. 4.11 shows that E2 addition does indeed result in substantially increased $R_{C T}$ values when the 35-mer aptamer is adsorbed on a flat Au electrode. The differential increase in $R_{C T}$ is substantially higher than the baseline response of a bare Au electrode at the lowest E2 concentration measured, $20 \mathrm{fM}$. Nyquist plots are provided in Appendix C. Fig. C.2.

\section{Sensing E2 with the adsorbed 35-mer aptamer on AuNP electrode}

The response of the AuNP electrode functionalised with non-specifically adsorbed 35-mer aptamer is shown in Fig. 4.12a. The diameter of semicircles in the Nyquist plot increased gradually as the concentration of E2 increased, reflecting an increased $\mathrm{R}_{\mathrm{CT}}$. By analysing the normalised change in $\Delta \mathrm{R}_{\mathrm{CT}} / \mathrm{R}_{\mathrm{CT}}{ }^{0}$ in Fig $4.12 \mathrm{p}$, it was found that there is a reproducible logarithmic correlation between the change in $\mathrm{R}_{\mathrm{CT}}$ and the concentration of $\mathrm{E} 2$ down to $1 \mathrm{fM}$ with a wide dynamic range up to $1 \mu \mathrm{M}$. It was confirmed that the $\mathrm{R}_{\mathrm{CT}}$ signal is a result of aptamer dissociation from the surface by using chronocoulometry to determine the amount of aptamer adsorbed on the surface before and after E2 exposure (Fig. 4.13). Exposure to 20 $\mu \mathrm{M}$ E2 resulted in $73 \%$ reduction in the 35-mer aptamer surface coverage. In the case of the AuNP electrode, aptamer dissociation from the surface upon E2 recognition could result in subsequent loss of the weakly connected AuNPs owing to the additional cross-linking role played by the aptamers described above. Provided that the aptamer responds specifically to its target, this additional effect may amplify the $\mathrm{R}_{\mathrm{CT}}$ increase for the AuNP electrode. 

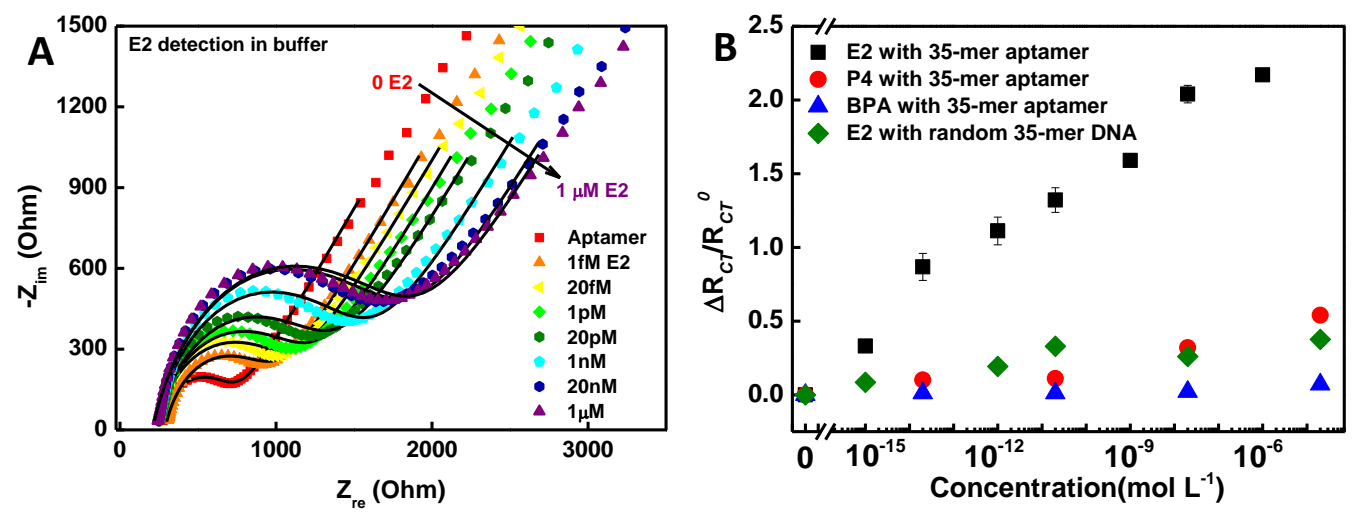

Figure 4.12: a) EIS spectra presented as Nyquist plots characterizing the response towards E2 concentrations with the 35-mer aptamer adsorbed on an AuNP electrode. Experimental data (symbols) are fitted to the Randles cell equivalent circuit (lines). b) Differential change in $\mathrm{R}_{\mathrm{CT}}$ vs. concentrations of E2, P4, and BPA for the 35-mer aptamer non-specifically adsorbed on an AuNP electrode. The response of a randomised 35-mer towards E2 is also shown as a control experiment. Error bars represent the range of two experiments.

\section{Specificity of sensing with the adsorbed 35-mer aptamer on AuNP electrode}

The specificity of the $\mathrm{R}_{\mathrm{CT}}$ response was investigated by changing both the aptamer probe and the analyte. The observed E2 sensitivity was not retained when the aptamer was replaced with a 35-mer oligonucleotide comprising the same bases but in a randomised sequence (the sequence of this oligonucleotide and other random sequences are provided in Appendix D, which therefore lacks the E2 binding domain. Chronocoulometry also confirms that the randomised sequence remains on the Au surface after exposure to E2 (Fig. 4.13). Using the E2 binding aptamer sequence, the sensing steps were repeated with structurally similar molecules progesterone and bisphenol-A. As shown in Fig $4.12 \mathrm{p}$, the 35-mer aptamer is remarkably specific, with only a baseline response to the potentially interfering molecules examined, even at concentrations as high as $1 \mu \mathrm{M}$. These findings show that the $\mathrm{R}_{\mathrm{CT}}$ signal results specifically from the 35-mer aptamer sequence binding with its target E2. The observed specificity is achieved as a result of the surface passivation established above, as well as the intrinsic specificity of this aptamer sequence. 


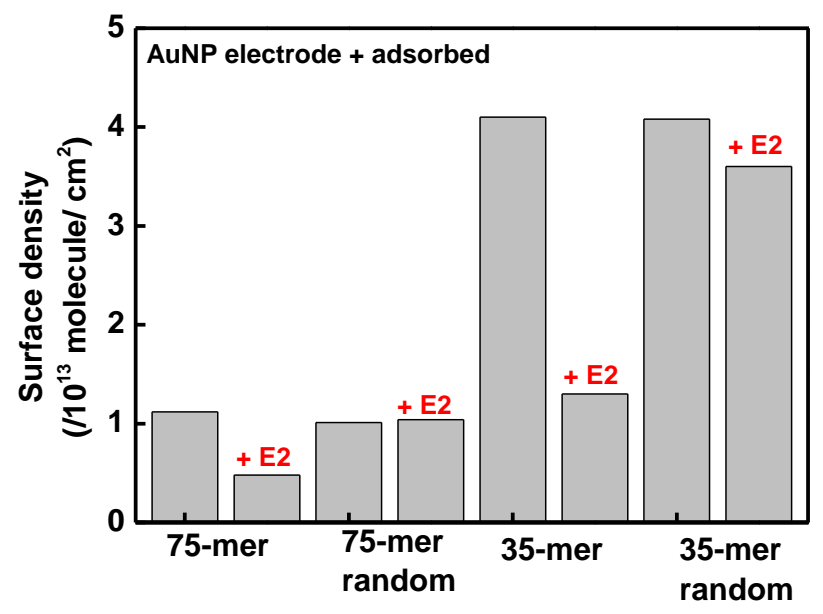

Figure 4.13: Chronocoulometry surface densities of adsorbed 75-mer aptamer and 35-mer aptamer before and after incubation with $20 \mu \mathrm{M}$ E2. The respective random DNA results are also shown.

Fig 4.12 shows that a simple and reliable electrochemical aptasensor can be constructed by simply adsorbing the desired aptamer onto an Au or AuNP and monitoring the EIS signals upon exposure to the test sample. The level of detection is over five orders of magnitude lower than the colorimetric E2 sensor based on a dispersion of AuNPs coated with exactly the same 35-mer aptamer sequence, Chapter 3 . Indeed, the reported level of detection is at least seven orders of magnitude lower than all previous colorimetric DNA (with $43 \mathrm{nM}$ level of detection $)^{42}$ and small molecule aptasensors ${ }^{43}$ based on DNA dissociation from AuNPs. This novel aptasensor platform provides a detection limit with one order of magnitude lower than previous electrochemical aptasensor based on EIS 6 68 and electrochemiluminescence ${ }^{175}$ as well as five order of magnitudes lower than fluorescence based methods. ${ }^{122[176}$ Unlike previous electrochemical small molecule aptasensors,, 17194161 the self-passivation characteristics of the non-covalently adsorbed aptamer system removes the necessity of further surface treatment to improve signal to noise ratio.

\section{Sensing E2 in spiked urine samples with the adsorbed 35-mer aptamer on AuNP electrode}

The $1 \mathrm{fM}$ level of detection in buffered solution is approximately five orders of magnitude lower than the recorded concentration of E2 in human urine. ${ }^{68 / 80} \mathrm{Hav}$ ing demonstrated the excellent level of detection and self-passivation properties 
of the sensor, the sensor's application in a more challenging physiological fluid of rat urine was examined. Fig. 4.14 shows that the sensor produces a very similar response towards E2 spiked into rat urine samples as was found for buffered water in Fig. 4.12p. Similarly, it was confirmed that the signals arose from specific interaction by the lack of response obtained from a sensor functionalised with the randomised 35-mer DNA, which confirms that the observed response in this complex matrix is specifically from the 35-mer aptamer. While it was previously demonstrated a colorimetric sensor using the same aptamer sequence in rat urine, the $5 \mathrm{nM}$ level of detection in that case exceeds the window of biological relevance.
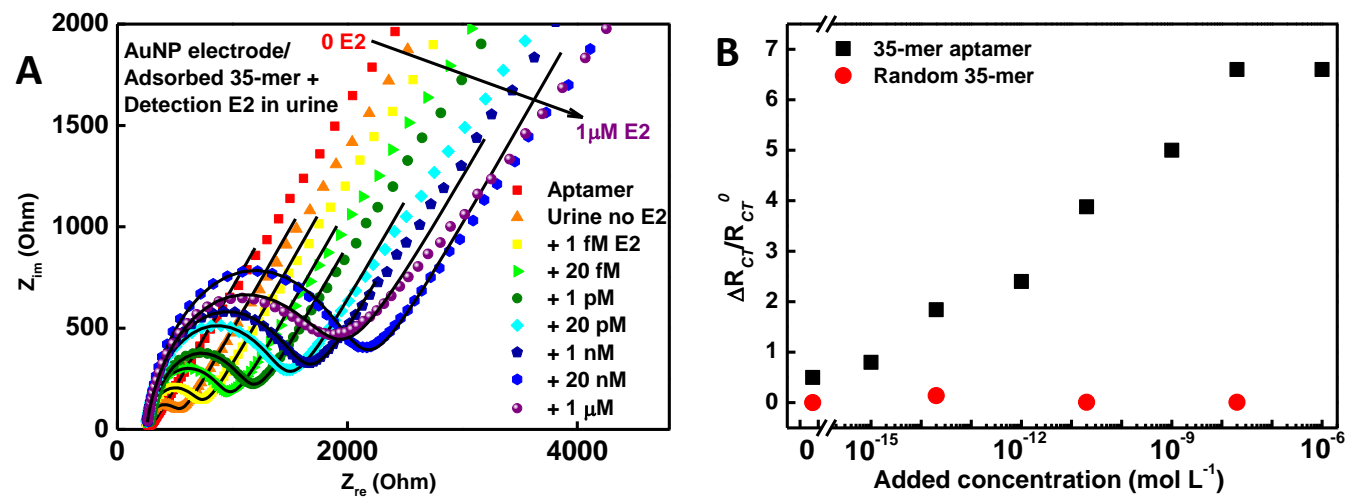

Figure 4.14: a) Nyquist plots of the 35-mer aptamer adsorbed on AuNPs and sequentially exposed to spiked rat urine with a range of E2 concentrations. b) Differential $\mathrm{R}_{\mathrm{CT}}$ as a function of $\mathrm{E} 2$ addition to rat urine. The response towards $\mathrm{E} 2$ is also shown for a control electrode with a random 35-mer DNA.

E2 sensing with adsorbed aptamers on AuNP electrode: A comparison between the 75-mer and 35-mer

The previous work in the colorimetric sensor format, Chapter 3, highlights the benefit of shortening aptamer sequences to facilitate their complete dissociation from AuNP surfaces upon binding to the target. The present sensor focuses on the shortened 35-mer, which lacks the primer nucleotides of the parent 75-mer E2 aptamer (with a $K_{\mathrm{D}}$ of $25 \mathrm{nM}$ ), and was found to produce an improved colorimetric sensor as a result. In order to determine whether the aptamer length dependence found for colorimetric sensors also applies to the present EIS sensor, the new AuNP EIS concept was applied to the 75-mer E2 aptamer. As shown in Fig. 4.15. the 75-mer system does exhibit a specific EIS response to E2, which is confirmed via a control experiment with randomised sequence and interfering molecules. However, the EIS signal towards E2 addition was significantly suppressed by a 
factor of 10 (based on the slope) in comparison with the 35-mer aptamer. This confirms that both methods ultimately depend upon the same initial step; dissociation of target-bound aptamers from Au surface. Additional conformation of this conclusion was obtained via chronolocoulometry measurements (Fig. 4.13) that show only $57 \%$ of the 75 -mer aptamer dissociates under saturation with E2 $(20 \mu \mathrm{M})$, compared with $75 \%$ for the 35 -mer.

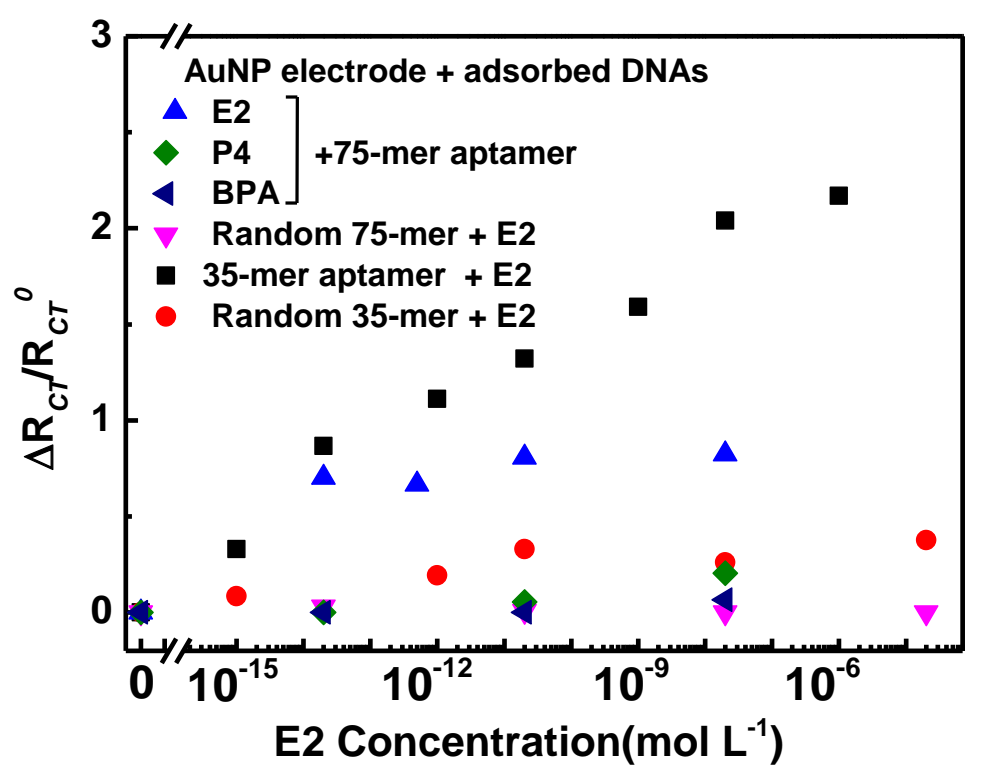

Figure 4.15: Differential change in $\mathrm{R}_{\mathrm{CT}}$ vs. concentrations of E2, P4, and BPA characterising the response of the 75-mer aptamer. The responses of the 35-mer aptamer, random 35-mer control, and random 75-mer control adsorbed onto AuNP electrodes towards E2 concentrations are also shown.

\subsection{Chapter conclusion}

This chapter presents the successful development and application of two EIS based aptasensor for the detection of E2. Conducting polymer based electrodes were used to tether the 75-mer aptamer and achieved a fM detection limit, wide dynamic range, robust operation, as well as evidence of discrimination against potential interfering agents. The sensor owes its exceptional sensitivity to the pronounced charge redistribution at the electrode interface when the aptamer folds around the neutral target molecule. This effect is amplified using a nanoporous conducting polymer electrode surface.

In the second approach, It was demonstrated that interfacial electrochemical signals are fundamentally different when DNA is coupled to Au surfaces via non- 
specific adsorption versus more conventional covalent tethering via a thiol linker. The greater surface contact required for DNA to adsorb to Au leads to a more lateral binding mode with a large fraction of the bases directly at the electrode interface - within the Debye screening length. This surface binding mode leads to displacement of the dense layer of ions found directly at the electrode interface at high potentials, and therefore enhanced charge transfer, and greater sensitivity to subsequent changes. The non-covalent binding mode was also shown to completely coat the Au surface and passivate it against other species that could interact with $\mathrm{Au}$ electrodes. In the case of AuNP electrodes, the non-covalent binding mode had the additional benefit of cross-linking stabilization. On the other hand, thiol tethered DNA perturbs the charge distribution further away from the interface, while leaving some exposed Au between the DNA strands that must be passivated to avoid spurious signals.

The pronounced EIS signals and the intrinsic passivation were exploited in the demonstration of a new sensor platform using a non-covalently adsorbed DNA on Au electrodes. Unlike previous EIS aptasensors that probe conformational changes, the present sensor resolves pronounced changes in $\mathrm{R}_{\mathrm{CT}}$ when targetbound aptamers dissociate from the Au electrode. The E2 aptasensor compares favourably with different formats of aptasensors. The present sensor had a femtomolar level of detection, a wide dynamic range, excellent discrimination against potential interfering agents, and showed robust operation in the complex physiological fluid of rat urine. The combination of strong electrochemical response, and intrinsic passivation, as well as simplicity, make this an appealing strategy for other DNA sensors and aptasensors. Moreover, the similarity to the operation of this EIS sensor and the established colorimetric sensor format may be useful for the dual development of methods that offer both visual detection, and sensitive electronic quantitation using the same aptamer systems.

\subsection{Experimental section}

Chemicals and Materials used in this Chapter can be found in Appendix D. 


\section{Electro-polymerisation of poly pyrrole-pyrroleacrylic acid and aptamer attach- ment}

The experiments related to electro-polymerisation and aptamer attachment were conducted by Bicheng $\mathrm{Zhu}$ according to previous reports as indicated below.

The electro-copolymerization of poly pyrrole (Py) and 3-pyrroleacrylic acid (PAA) was carried out by using a Bio-Logic instrument (Bio-Logic SP-300) at a constant potential of $1.0 \mathrm{~V}(\mathrm{Ag} / \mathrm{AgCl})$ with $0.035 \mathrm{mC}$ passed in a solution containing 0.005 M Py, 0.2 mM PAA and poly(4-styrenesulfonic acid) solution in deionized water (Milli-Q, $18.2 \mathrm{M} \Omega \mathrm{cm}$ ) as previously described by Zhu et al. ${ }^{149}$ Amino-modified aptamer attachment was carried out by incubation of the poly(Py-co-PAA) modified GCE for $1 \mathrm{~h}$ at $28^{\circ} \mathrm{C}$ using EDC/NHS chemistry as previously described. 143 The immobilization solution comprised $20 \mu \mathrm{L}$ PBS buffer containing $1.5 \mu \mathrm{M}$ aptamers and $80 \mathrm{mM}$ EDC and $120 \mathrm{mM}$ NHS. Before attachment, the aptamer was heated at $70{ }^{\circ} \mathrm{C}$ for $5 \mathrm{~min}$ (for denaturation). After attachment, the electrode was washed three times by PBS solution to remove any non-specifically adhered aptamer.

\section{Formation of AuNP based electrodes}

A dense layer of AuNPs was electrochemically deposited on the surface of a glassy carbon electrode (GCE) or a gold electrode (where noted) as previously reported. $\frac{168}{}$ Briefly, a three-electrode cell with a volume of $5 \mathrm{~mL}$ was used, comprising a polished and cleaned glassy carbon working electrode (GCE) (eDAQ, $1.0 \mathrm{~mm}$ in diameter), or gold electrode (10 mm in diameter), $\mathrm{Ag} / \mathrm{AgCl}(3 \mathrm{M} \mathrm{NaCl}$, $+0.197 \mathrm{~V}$ vs. standard hydrogen electrode (SHE)) reference electrode and Pt wire counter electrode (using Bio-Logic SP-300 instrument). The Au deposition was done by immersing the electrodes in a $5 \mathrm{~mL}$ solution containing $1 \mathrm{mM} \mathrm{HAuCl}_{4}$, $0.01 \mathrm{M} \mathrm{Na}_{2} \mathrm{SO}_{4}$ and $0.01 \mathrm{M} \mathrm{H}_{2} \mathrm{SO}_{4}$ at a constant potential of - $0.2 \mathrm{~V}(\mathrm{Ag} / \mathrm{AgCl})$ for $30 \mathrm{~s}$. Before deposition, the GCE or gold electrode was polished with 0.5 $\mu \mathrm{m}$ alumina slurry, followed by electrochemical cleaning by subjecting to potential cycling (three times at a scan rate of $20 \mathrm{mV} / \mathrm{s}$ ) between -1.0 and $1.0 \mathrm{~V}$ in $0.25 \mathrm{M} \mathrm{H}_{2} \mathrm{SO}_{4}$, washing with acetone, ethanol and deionized water (Milli-Q, 18.2 $\mathrm{M} \Omega \mathrm{cm})$. All solutions were de-gassed for $15 \mathrm{~min}$ using $\mathrm{N}_{2}$ gas. 


\section{Scanning electron microscopy (SEM) surface characterization}

These experiments were conducted by Bicheng Zhu. The electrodes were sputtered with platinum in order to increase the conductivity. Sample stage was tilted $30^{\circ}$ before scanning with Philip ML30S FEG Scanning Electron Microscope.

\section{DNA attachment to Au based surfaces}

For the thiolated and non-thiolated DNAs, the surface of the electrode was rinsed thoroughly with $10 \mathrm{mM}$ trisodium citrate buffer, $\mathrm{pH} 3$, and $0.1 \mathrm{nmol}$ of the DNAs (16.7 $\mu \mathrm{M}$, in $5 \mathrm{mM}$ trisodium citrate buffer, $\mathrm{pH}$ 3) was incubated with the $\mathrm{Au}$ based surface for $20 \mathrm{~min}$. This protocol was found to produce quantifiable monolayers of Au-S DNAs in 3 min on AuNPs ${ }^{177}$ and was adopted here (a control experiment via exposing the aptamers on GCE resulted in no adsorption, refer to Figure C.3). Treatment with 6-mercaptohexanoic acid, $\mathrm{SH}-(\mathrm{CH} 2)_{5} \mathrm{COOH}$, (MHA) was carried out under the same condition as coupling the DNA but with a concentration of $10 \mathrm{pM}$. Bovine serum albumin (BSA) was dissolved in deionized water (Milli-Q, $18.2 \mathrm{M} \Omega \mathrm{cm}$ ) at a concentration of $0.8 \mathrm{mM}$ and exposed to the surface of AuNP electrodes for $20 \mathrm{~min}$.

\section{Target sensing}

Detection of E2 and interfering molecules (P4) progesterone, (BPA) bisphenol-A, was achieved by incubating the DNA-modified electrode in $30 \mu \mathrm{L}$ binding washing buffer solution (BWB with $5 \%$ ethanol) containing the desired concentration of the target for $15 \mathrm{~min}$ at room temperature. Before EIS or subsequent measurements, the electrode was washed three times by using BWB to remove any nonbound molecules of the targets. E2 detection in rat urine was the same as above except that E2 was spiked in rat urine after adjusting the content of ethanol to $5 \%$, for solubility reasons. Rat urine was collected from sexually mature ship rats (Rattus rattus), then filtered with $0.22 \mu \mathrm{m}$ syringe-filters (control rat urine sample comprised blank rat urine containing 5\% ethanol). Rat urine was collected by Dr. Christine Stockum, from the School of Biological Sciences, Victoria University of Wellington. 


\section{EIS measurements}

EIS measurements were recorded in a three-electrode cell containing $5 \mathrm{~mL}$ PBS solution with $5.0 \mathrm{mM}$ of $[\mathrm{Fe}-(\mathrm{CN}) 6]^{3-/ 4-}(1: 1, \mathrm{~mol}: \mathrm{mol})$ at an applied bias of $0.230 \mathrm{~V}(\mathrm{Ag} / \mathrm{AgCl})$. EIS measurements were recorded on a Bio-Logic instrument (Bio-Logic SP-300). EIS measurements were carried on with a $10 \mathrm{mV}$ sinusoidal amplitude and collected for harmonic frequencies between $100 \mathrm{mHz}$ and $100 \mathrm{kHz}$ at 12 steps per decade and analysed using the Randles equivalent circuite (known as Zfit) shown in Fig. 4.1 .

\section{DNA surface densities}

DNA surface densities on AuNP electrodes were determined using the chronocoulometry (CC) method developed by Steel et al. ${ }^{[133}$ The attached DNAs (through $\mathrm{Au}-\mathrm{S}$ or non-specifically adsorbed) on AuNP electrode were first immersed in a low ionic strength electrolyte, $10 \mathrm{mM}$ tris- $\mathrm{HCl}$ buffer at a $\mathrm{pH} 7.4$, the potential stepped from 200 to $-500 \mathrm{mV}$ versus $(\mathrm{Ag} / \mathrm{AgCl}$ ) for $500 \mathrm{~ms}$ (using BAS 100A electrochemical analyser), and the resulting charge flow was measured. The electrode was then immersed for $20 \mathrm{~min}$ in a solution of $150 \mu \mathrm{M} \mathrm{Ru}\left(\mathrm{NH}_{3}\right)_{6}{ }^{3+}$ (RuHex) in $10 \mathrm{mM}$ tris- $\mathrm{HCl}$ buffer at a $\mathrm{pH} 7.4$, and the measurement repeated. By plotting the charge $Q$ versus the square root of time $\left(t^{1 / 2}\right)$, the excess of RuHex was determined from the difference in $y$-intercepts for such plots and was related to the DNA surface density. 


\section{Chapter 5}

\section{Evaluation and comparisons of the developed aptasensors}

This chapter focuses on providing a critical evaluation of the developed aptasensors by comparing their performance, advantages and disadvantages, along with considering factors that might impact their performance and their potential applications. In addition, this chapter provides comparisons between the developed aptasensors and methods available in the literature for the detection of E2, including aptasensors that used a different aptamer developed by Kim et al. ${ }^{7}$

\subsubsection{Comparison between the developed aptasensors}

In this thesis, several novel aptasensors have been developed and applied for the detection of E2. The three aptasensors demonstrated here are; size based detection (with a detection limit of $5 \mathrm{nM}$ ), AuNP based colorimetric detection (with a detection limit of $200 \mathrm{pM}$ ), and EIS based detection using conducting polymer and gold based electrodes (with a detection limit of $1 \mathrm{fM}$ ).

The colorimetric and EIS aptasensors possess the advantages of simplicity, sensitivity, rapidity, and availability, with no extensive sample pre-treatment steps prior to the detection of E2, even in complex biological samples.

Compared to the EIS aptasensor, the colorimetric based detection using AuNP is more suitable for field screening of E2 residue where no additional instrumentation is required. However, proper control experiments should be carefully 
designed and performed to evaluate the non-specific aggregation induced from high ionic strength samples or from any substance present in the sample. Recommended control experiments include performing parallel sample analysis with AuNPs coated with a random sequence that lacks the ability to bind E2.

EIS aptasensors using conducting polymer and AuNP electrodes afford a detection limit of five orders of magnitude higher than the colorimetric method and a dynamic range that spans nine orders of magnitude of E2 concentration. EIS based aptasensors are able to operate in complex biological matrices. In fact both EIS methods have the characteristic of self-passivation where no need for further backfilling with small molecules. This method could be applied for accurate determination of E2 after comparison against calibration curves of the same analyte. Achieving the low level of detection and wide dynamic range demands instrumentation (an impedance analyser), which makes the method more suitable for lab-based analysis than in situ sensing. It is recommended to evaluate the obtained results against data obtained with a control non-specific DNA to ensure the specificity of the observation. Filtration of the samples with $\mu \mathrm{m}$ filters is recommended prior to the analysis with either colorimetric or EIS aptasensors to remove large objects present in the sample.

Although the approaches to sense E2, using AuNP colorimetric and EIS based assays, have achieved high sensitivity and good discrimination, the practicality and "real" detection limits of such sensors with real samples can be different. While detection levels were quoted according to where a reliable signal was resolved above the level of a clean control, real world samples are complex with many variables under less control than laboratory based testing, and indeed unknown sample composition. The presence of contaminants, interfering agents and the fluid complexity could cause a background (i.e., non-specific signal), which can limit the real level of detection. The real level of detection is where the signal of the target analyte exceeds the background from other possible interfering agents. This makes it imperative to comprehensively characterize the range of possible background signals, as well as continuing to improve the specificity of aptasensor devices.

With regard to the size based aptasensor, the success of this strategy is ensured by the use of NPs of a suitable size and excellent monodispersity combined with their inert characteristic towards interaction with the backbone of the aptamer sequences. These factors enable resolving increase in size by TRPS and DLS upon functionalizing the probe to NPs followed by monitoring target recognition. An additional requirement for TRPS is that the use of an electrolyte with a reasonable 
conductivity that allows accurate size measurements. Given these limitations, the size based sensor could be suitable for estimating E2 concentration after extraction from the original sample environments. Better detection limits (currently 5 $\mathrm{nM}$ ) could be achieved with aptamers that have better affinities. In addition to sensing, the developed method could potentially be used to guide the synthetic evolution of aptamers to attain better binding affinity and specificity. The size based aptasensor also proved useful in guiding the design of the EIS sensor, since both sensors probe the target-induced folding of the aptamer.

Overall, the sensor templates developed in this thesis are label-free that do not require explicit knowledge of the bound and unbound aptamer conformations. They only require that binding induces some kind of conformational change that is a general feature of small molecule binding aptamers. Therefore, the sensor designs in this thesis are considered to be broadly applicable to other aptamers targeting small molecules.

Compared to traditional methods including chromatographic techniques, the developed aptasensors may help regulatory agencies, medical patients, and other entities to monitor and to screen real samples for the presence of E2 with low cost and less effort.

\subsubsection{The role of excess flanking nucleotides in enhancing or sup- pressing the sensing signals}

It was found in the colorimetric sensor format that the observed difference in signal sensitivity between the 75-mer and 35-mer aptamers could be partially related to the lesser degree of surface dissociation when the longer aptamer binds to E2. Comparing the colorimetric and EIS-adsorbed aptamer on Au surface responses for the same pair of aptamer sequences to the E2 target confirms that both methods ultimately depend upon the same initial step; dissociation of target-bound aptamers from Au surface. However, EIS affords over 5 orders of magnitude enhancement in the sensitivity of detecting the dissociation of aptamers adsorbed on Au surfaces compared with the colorimetric method.

In contrast, the opposite trend was observed with EIS-based conducting polymer. The 75-mer aptamer coupled through an amide bond was found to produce a stronger E2 signal than the shorter 35-mer aptamer. Excess nucleotides (20 bases at each end of the core 35-mer) could bring more charges to the double layer 
region and enhance $R_{C T}$ signals when forming aptamer-target complex. It might be even advantageous for this type of sensors to add additional bases to enhance the signals, provided that the binding region is not too far from the surface.

A less robust comparison can be made between the performance of the 75-mer E2 aptamer and the 27-mer adenosine aptamer in the size based sensor using TRPS. Long sequences, that have minimum self-complementarity like the 75-mer E2 aptamer, are likely to assist resolving the initial increase in size when functionalized to NPs using an amide bond. Additionally, long aptamer sequences could also allow establishing a relatively wide dynamic range (the aptamer $K_{\mathrm{D}}$ could play a role here) of the target that falls within the resolution of TRPS. On the other hand, short sequences as adenosine aptamer, or sequences with maximum selfcomplementarity, are likely to be less efficient with the size based sensor format when quantitative analysis is required. It is expected that qualitative measurement (yes/no type of analysis) will be efficiently obtained as demonstrated with adenosine aptamer.

In summary, SELEX isolates sequences for affinity. However, a good binder does not mean a good reporter. Engineering the structure of the aptamer, post selection, is a crucial tool that should be exploited in aptasensors with particular regard to the way signals are transduced.

\subsubsection{Aptasensors developed in this thesis versus literature meth- ods}

The advantages of EIS based aptasensors system (using conducting polymer and adsorbed aptamer onto Au surface) are apparent in the comparison with other E2 sensors shown in Table 5.1, It can be seen that the detection limit of EIS based sensor ( $1 \mathrm{fM}$ ) is substantially better than most other sensors and rivalled by only two methods (EIS based electrode coated with dendritic $\mathrm{Au}^{[178}$ and Photoelectrochemical with $\mathrm{TiO}_{2}$ nanotube arrays ${ }^{179}$ ). Moreover, the nine orders of magnitude dynamic range reported here is significantly wider than all reported E2 aptasensors, with most only operating within 2 to 3 orders of magnitude of their level of detection. The present study provides the only existing colorimetric assay for E2 with a 200 pM level of detection and operation in urine samples. The concentration of E2 is directly related to EIS and colorimetric signals (i.e., not based on a competitive assay). Previous work such as electrochemiluminescence with a complementary DNA sequance, ${ }^{175}$ fluorescence resonant energy transfer with 
QD, ${ }^{176}$ or fluorescence based detection ${ }^{122}$ (indicated in Table 5.1) reported E2 concentration indirectly based on competitive assays that add additional steps to the sensor preparation and could potentially effect the sensor sensitivity.

Table 5.1: Comparisons of various methods for the detection of E2 including aptasensors developed in this thesis.

\begin{tabular}{|c|c|c|c|c|c|}
\hline \multirow{2}{*}{ Method } & \multirow{2}{*}{ LOD (M) } & \multirow{2}{*}{ Dynamic range $(\mathrm{M})$} & \multicolumn{2}{|c|}{ Tested samples } & \multirow{2}{*}{ Ref. } \\
\hline & & & Bio. & Enviro. & \\
\hline Enzyme-linked immunosorbent assay & $2 \times 10^{-11}$ & $4 \times 10^{-11}$ to $4 \times 10^{-8}$ & No & Yes & 180 \\
\hline EIS based electrode coated with dendritic Au & $5 \times 10^{-15}$ & $1 \times 10^{-14}$ to $1 \times 10^{-9}$ & No & Yes & 178 \\
\hline Electrochemiluminescence with a complementary DNA & $1 \times 10^{-12}$ & $1 \times 10^{-11}$ to $1 \times 10^{-8}$ & Yes & Yes & 175 \\
\hline Hybridised Au electrode probed with SWV ${ }^{1}$ & $2 \times 10^{-11}$ & $1 \times 10^{-10}$ to $1 \times 10^{-7}$ & No & No & 121 \\
\hline Fluorescence resonant energy transfer with a QD & $2 \times 10^{-10}$ & $8 \times 10^{-10}$ to $2 \times 10^{-8}$ & No & Yes & 176 \\
\hline EIS on flat Au electrode & $2 \times 10^{-12}$ & $1 \times 10^{-11}$ to $1 \times 10^{-8}$ & Yes & No & 68 \\
\hline Fluorescence based detection & $2 \times 10^{-9}$ & $2 \times 10^{-9}$ to $8 \times 10^{-8}$ & No & Yes & 122 \\
\hline Au electrode probed with CV and SWV & $2 \times 1^{-10}$ & $2 \times 1^{-10}$ to $1 \times 10^{-8}$ & No & No & 7 \\
\hline Photoelectrochemical with $\mathrm{TiO}_{2}$ nanotube arrays & $3 \times 10^{-15}$ & $5 \times 10^{-14}$ to $2 \times 10^{-11}$ & No & Yes & 179 \\
\hline Size based aptasensor & $5 \times 10^{-9}$ & $5 \times 10^{-9}$ to $1 \times 10^{-7}$ & No & No & Present ${ }^{23}$ \\
\hline AuNP colorimetric aptasensor & $\mathbf{2} \times 10^{-10}$ & $\mathbf{2} \times 10^{-10}$ to $8 \times 10^{-10}$ & Yes & Yes & Present 181 \\
\hline EIS based adsorbed aptamer on gold surface & $\mathbf{1} \times 10^{-15}$ & $\mathbf{1} \times \mathbf{1 0}^{-15}$ to $\mathbf{1} \times \mathbf{1 0}^{-6}$ & Yes & No & Present \\
\hline EIS-based conducting polymer & $\mathbf{1} \times 10^{-15}$ & $\mathbf{1} \times 10^{-15}$ to $1 \times 10^{-6}$ & Yes & Yes & Present ${ }^{182}$ \\
\hline
\end{tabular}

${ }^{1}$ Square wave voltammetry 


\section{Chapter 6}

\section{Conclusions and Future work}

\subsection{Conclusions}

The objectives of this thesis to develop various aptasensors for the detection of E2 listed in Chapter 1 were set out as follows: 1) using a label free size based format that does not require detailed knowledge of the aptamer target-induced conformation, 2) developing a colorimetric AuNP assay and understanding the effect of the length of aptamer sequences on their dissociation from AuNP surfaces and subsequent aggregation, 3) developing ultrasensitive EIS-based sensors using a conducting polymer and non-specifically adsorbed aptamer onto gold surfaces, and 4) comparing advantages and disadvantages of each signal transduction mechanism.

The key achievements and contributions of the thesis are discussed below:

A new label free aptasensor scheme was developed. It features the E2 aptamer covalently tethered to carboxylated polystyrene NPs as the signal carrier. It was proven that simple methods for measuring NP size and surface potential, as DLS and TRPS, can be used to register binding events when the aptamer adopts a more tightly folded conformation around its target. The sensor is able to detect E2 as low as $5 \mathrm{nM}$ in buffered solution. Unlike methods that depend on labelling different sections of the aptamer with pairs of fluorescent dyes or with a redox probe, this new method is able to couple conformational switching to binding signals and does not require explicit knowledge of the bound and unbound aptamer conformations. 
AuNPs were previously exploited in the construction of colorimetric aptasensors for different targets. However, it was unknown how the length of aptamer sequences affects their dissociation from AuNP surfaces and subsequent aggregation. Starting from the 75-mer aptamer, the sequence was truncated to generate 35- and 22-base variants. The resulting 200 pM limit of detection performance of 35-mer and 22-mer aptamers surpasses existing colorimetric aptasensors and the improvement was attributed a slightly improved aptamer affinity for E2 when the flanking nucleotide sequences were eliminated and suppressed residual affinity of target-bound aptamers to AuNPs. Close comparison of AuNP surface interactions between the 35-mer and 22-mer showed that a small number of excess bases are required in order to achieve the optimal balance of affinity to AuNPs and the target. The benefit of eliminating redundant bases from the aptamer sequences used in AuNP assays was illustrated in the first demonstration of a colorimetric AuNP sensor in complex biological urine samples.

An EIS aptasensor for E2 with one femtomolar detection limit, wide dynamic range, and robust operation in buffer solutions and urine samples was initially demonstrated with the E2 aptamer functionalized via amide bonds to a conducting polymer electrode. E2 binding causes a redistribution of negative charges in the electrode double-layer region when the aptamer adopts a folded conformation around the small neutral target molecule. Additionally, as a novel alternative approach, sensing electrodes were also created via the non-specific adsorption of the 35-mer onto Au and AuNP electrodes. This approach led to the same level of detection as the conducting polymer aptasensor, but via a mechanism with similarities to the colorimetric sensor. Non-specific adsorption of aptamers to Au was found to play additional favourable roles including self-passivation and stabilization of AuNP based electrodes. Sensing with this format might remove the need for laborious surface passivation with alkylthiol molecules encountered with the conventional covalent attachment of the DNAs through thiol-linkers.

Overall, this thesis provides, for the first time, understanding of aptasensors by comparing the performance of the same aptamer in various sensing platforms. Long aptamers sequences appeared to be more efficient in signal transduction when specific surface tethering is involved, as in the size-based assay, and the electrochemical assay with aptamers covalently tethered to the electrode. Here, the non-binding flanking nucleotides adjacent to the target binding pocket appeared to amplify the sensing signals. However, shorter truncated sequences showed better performance when signal generation depends on surface dissociation of non-specifically adsorbed aptamer sequences, as in the colorimetric assay, and the electrochemical sensor constructed from adsorbed aptamers. These in- 
sights can be readily applied to aptasensors for the growing range of targets.

\subsection{Future work}

Understanding the effects of long term storage on the response of the developed aptasensors will aid their potential availability for end-users including regulatory laboratories or even ordinary users. One particular aspect that should be investigated is that whether aptamers retain their binding functions after long term contact with AuNPs. This could be achieved through investigating the sensing responses towards the target over different time intervals of a given period.

The sensitivity of EIS-based conducting polymer could be enhanced even more by investigating the addition of various number of bases near the core region (stem-loop structure illustrated in Chapter 3) and at various ends.

Despite efforts made in this thesis to improve the specificity, the 35-mer aptamer showed low reactivity towards potential interfering molecules, progesterone and testosterone, in the colorimetric sensor. Additionally, EIS based aptasensors showed low responses to progesterone at high concentrations when the 75-mer and 35mer aptamers were used. These molecules could be present in the detected samples at levels that might significantly exceed E2 concentrations and consequently result in an overestimated determination of the target. Therefore, there is a clear motivation to develop aptamers with absolute specificity to E2. Aptamers with high specificity can be isolated by SELEX which can even discriminate between enantiomers (molecules that are mirror images of one another). For example, Grozio et al. $\frac{183}{1}$ isolated ssDNA aptamers that bind the small hormone abscisic acid and are able to discriminate between the target enantiomers.

Future research should also focus on developing more robust and high throughput methods for determining the $K_{\mathrm{D}}$ values of aptamers for small molecules. Equilibrium separation method is not always suitable for the $K_{\mathrm{D}}$ measurements due to non-specific adsorption of the target on the nitrocellulose filters that are commercially available. One possible way to solve this issue is by developing a better separation method. The aptamer of interest could be immobilised on magnetic particles that will facilitate the separation of the bound and unbound fractions of the target. Alternatively, a fluorescently labelled short complementary sequence could be hybridised to a certain section of the aptamer. Upon target recognition and after magnetic-based separation, the fluorescence of the comple- 
mentary sequence free in solution can be measured (after dissociation from the aptamer) and related to the target concentration.

Additionally, this thesis developed sensor formats that could be adapted for different aptamers targeting different small molecules. A number of aptamers that have high affinity and specificity for their targets are already available in the literature, including bisphenol A, $\stackrel{111}{r}$ cortisol, $\stackrel{184}{=}$ progesterone,,$\frac{185}{1}$ and others. If the ideas expressed in this thesis can be shown to have sufficient generality to different targets, they may also be readily applied to new aptamer sequences in the future.

Multiplexed methods for the simultaneous detection of these targets and others ('fingerprint' identification) could also be developed. In a multiplexed sensor array, it may be possible to better account for background interference if the likely interfering agents were also quantified. Moreover, one could imagine profiling numerous members of the same class of compounds, for example steroidal hormones, or estrogen mimics.

Previous work in the literature showed that lateral flow based assays are inefficient for the detection of small targets due to their inherent lack of complex structures (reviewed in Chapter 1). However, the aptamer dissociation from the surface of AuNPs upon target recognition could be exploited to develop a novel lateral flow assay that depends on probing the presence or absence of the aptamer on the surface. Proteins, including bovine serum albumin (BSA), can be immobilised onto nitrocellulose membranes and they have strong interaction with AuNPs. ${ }^{186}$ The presence of aptamers on the surface of AuNPs screens the interaction between the AuNPs and BSA as demonstrated in Chapter 4. It is expected that the detection of E2 which is associated with the departure of aptamers from the surface will result in an easily fabricated lateral flow assays. 


\section{Appendix A}

\section{Supplementary information of size based sensor}

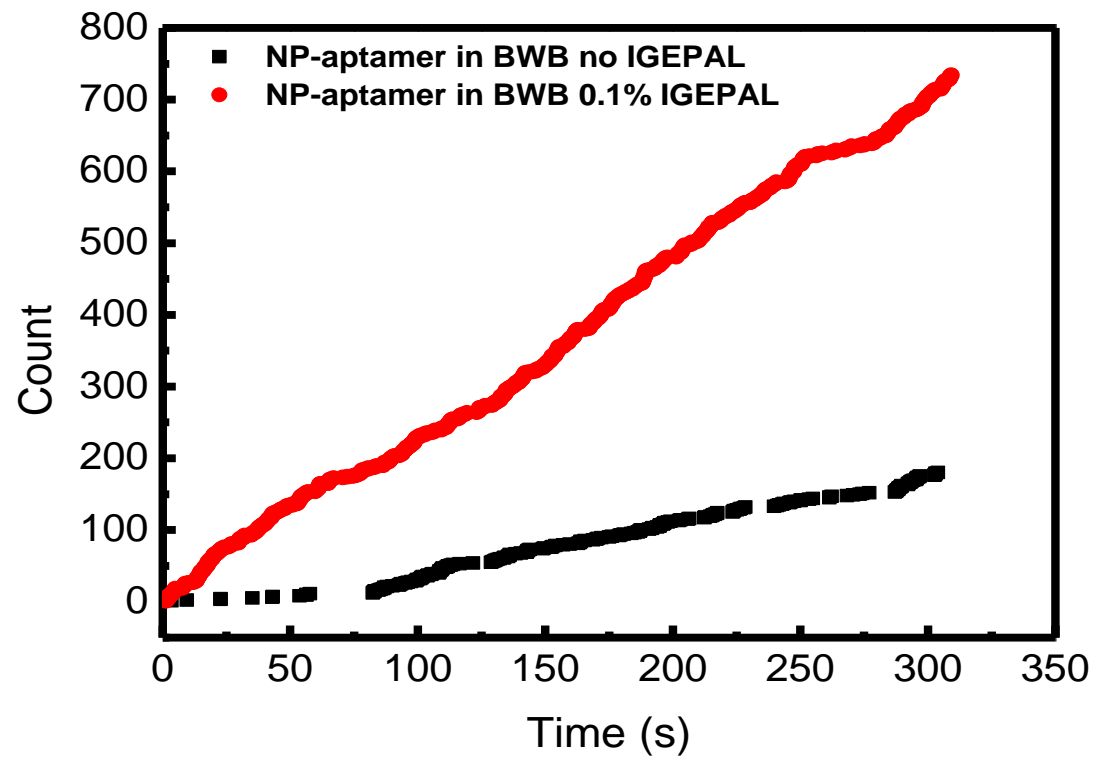

Figure A.1: The effect of IGEPAL on the NP-aptamer count rate in TRPS. Samples that do not contain IGEPAL do not properly wet the pore, resulting in slow throughput and partial blockages. 


\section{Appendix B}

\section{Supplementary information of the colorimetric sensor}

\section{B.1 Detection of E2 using the 75-mer aptamer, 35-mer aptamer, and 22-mer aptamer}

\section{B.1.1 Spectra for aptamer responses to E2 concentrations}
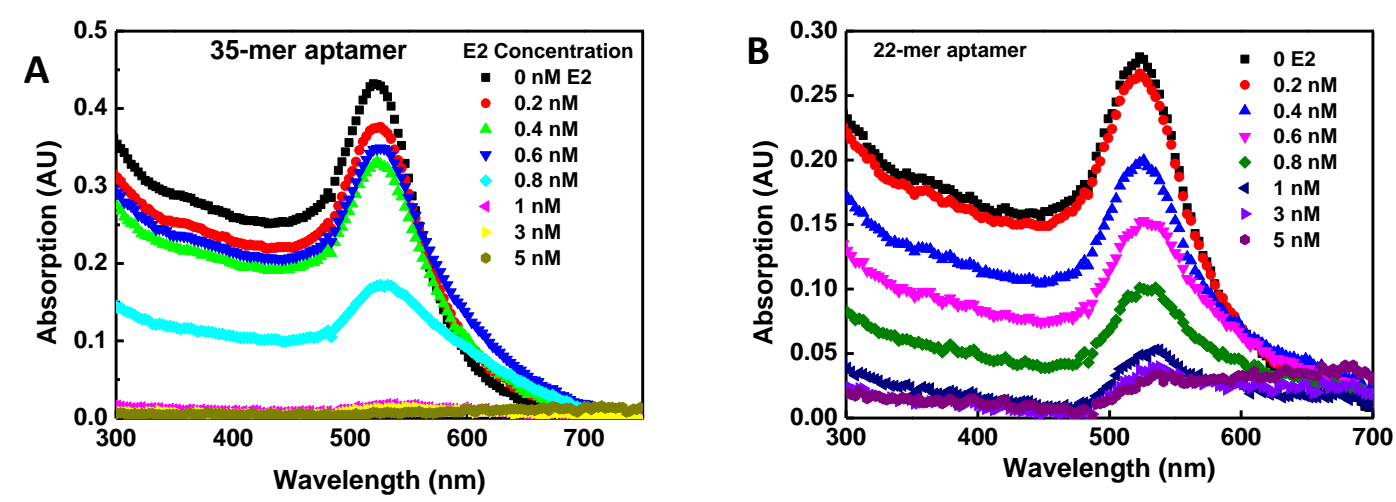

Figure B.1: Absorption spectra of E2 detection samples using a) 35-mer aptamer, and b) 22-mer aptamer. 


\section{B.1.2 Specificity examination}
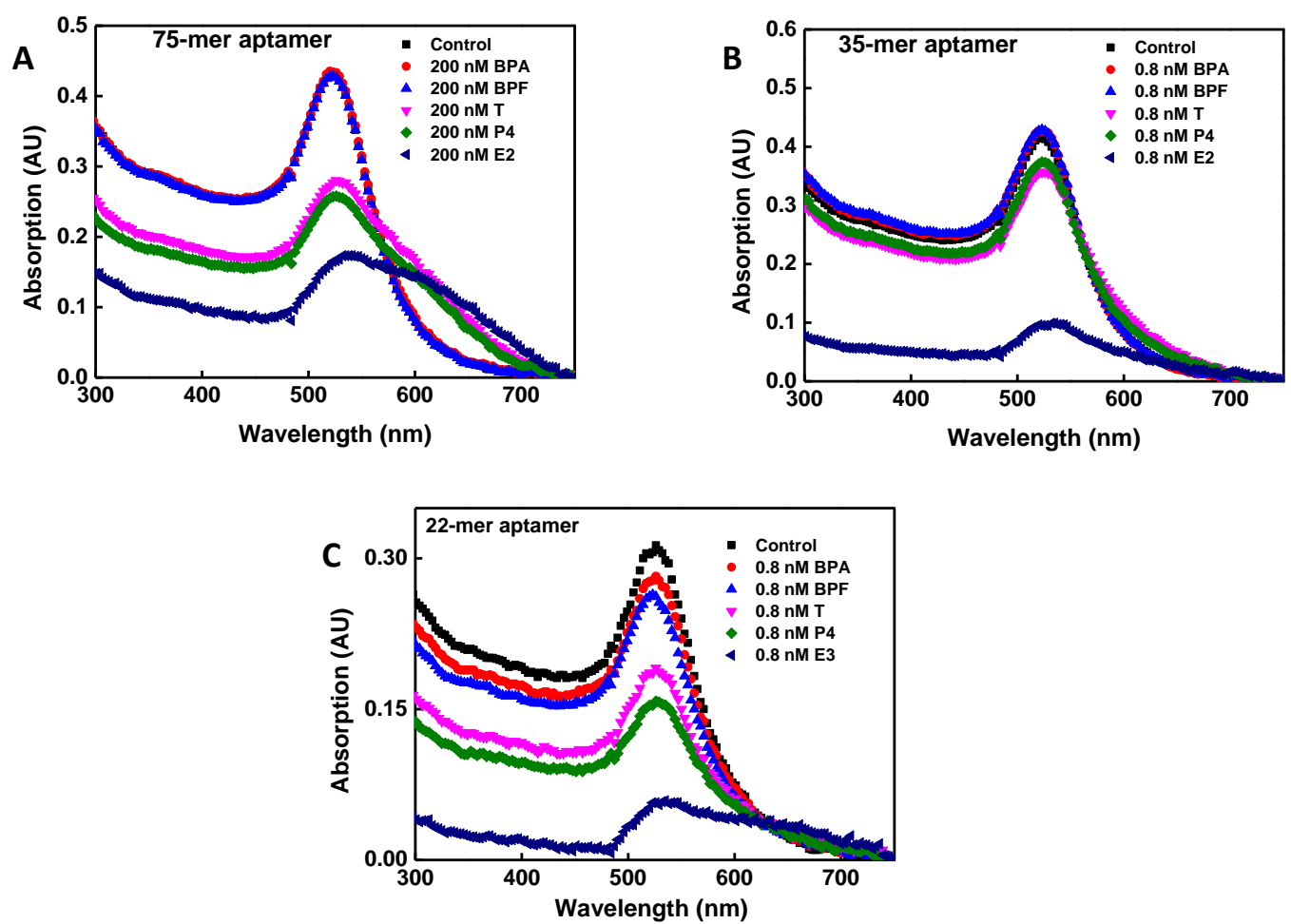

Figure B.2: Representative UV-vis absorption spectra obtained during the specificity examination using a) 75-mer aptamer with $200 \mathrm{nM}$ target concentration, b) 35-mer aptamer and c) 22-mer aptamer with $0.8 \mathrm{nM}$ target concentration. 


\section{B.2 CD characterisation of E2 binding with 75-mer aptamer, 35-mer aptamer, and 22-mer aptamer and when replacing the flanking nucleotides with Poly- T20}

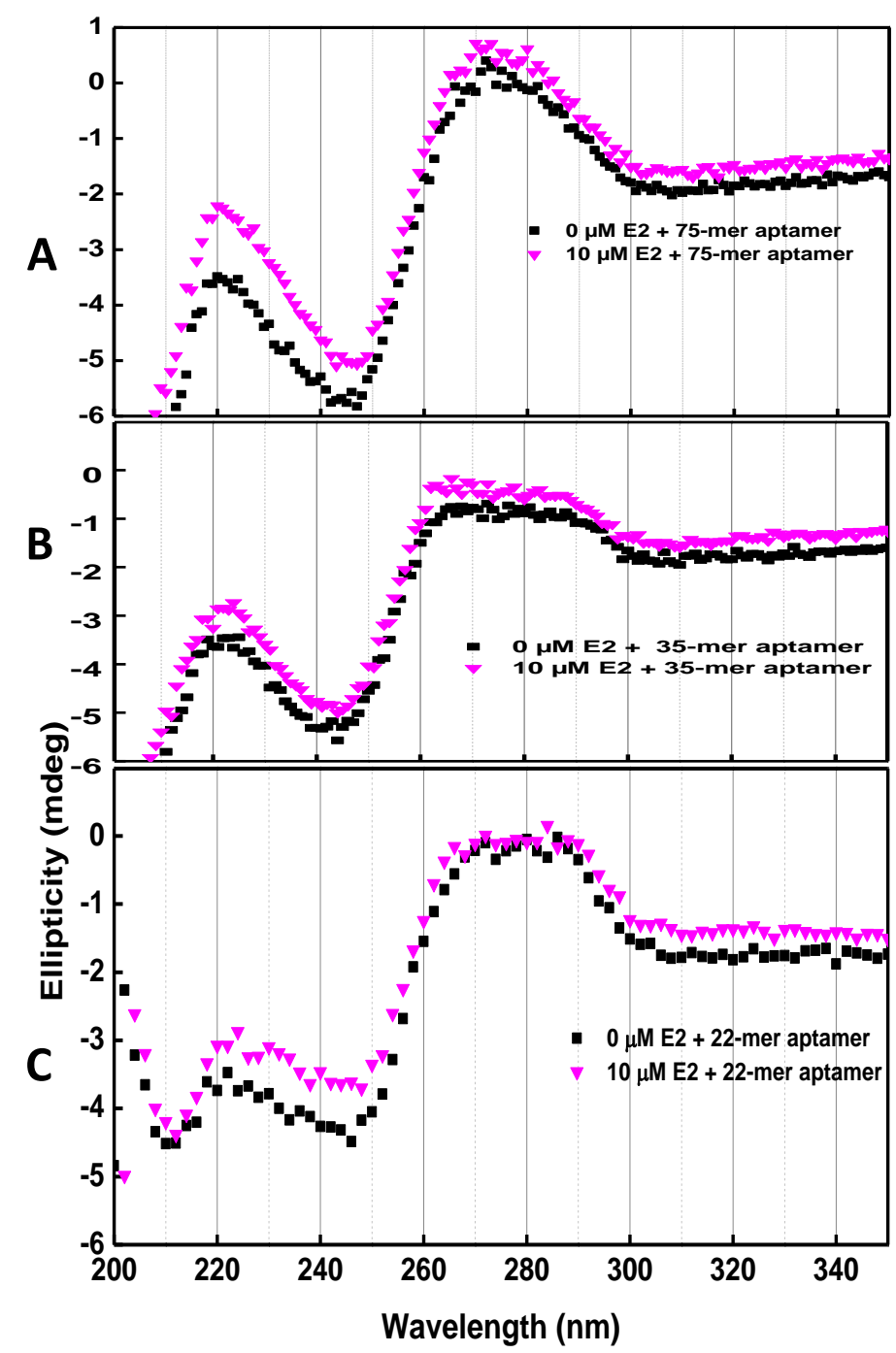

Figure B.3: CD characterisation of E2 $(10 \mu \mathrm{M})$ binding with a) $400 \mathrm{nM}$ of 75 -mer aptamer , b) $600 \mathrm{nM}$ of 35-mer aptamer, and c) $600 \mathrm{nM}$ of 22-mer aptamer. 


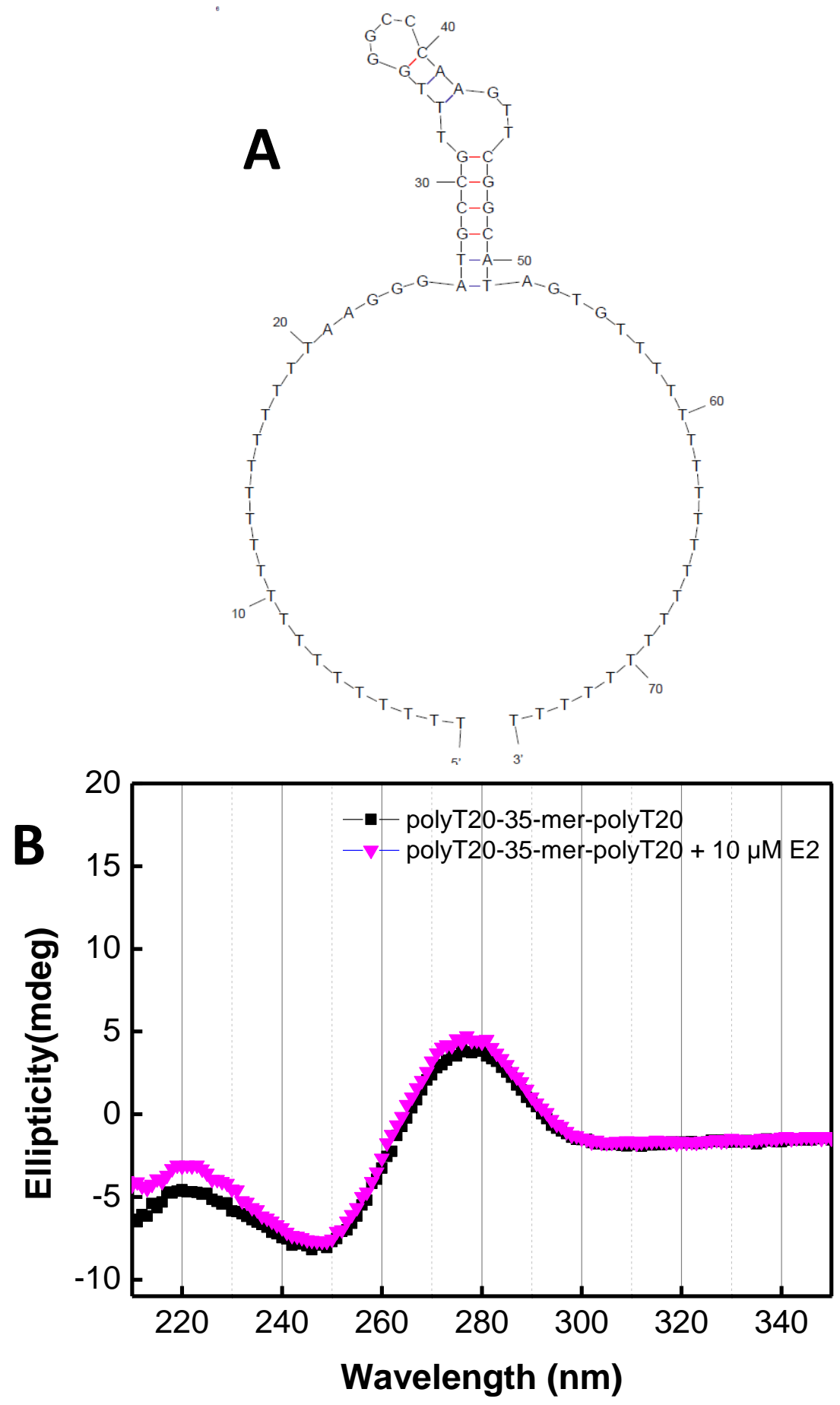

Figure B.4: a) Secondary structure of the poly T20-(35-mer)-poly T20 DNA predicted by M-fold program. b) CD spectra for $400 \mathrm{nM}$ of the T20-(35-mer)-poly T20 DNA incubated with 0 and $10 \mu \mathrm{M}$. 


\section{B.3 Urine samples: Determination of ionic strength and detection of E2}

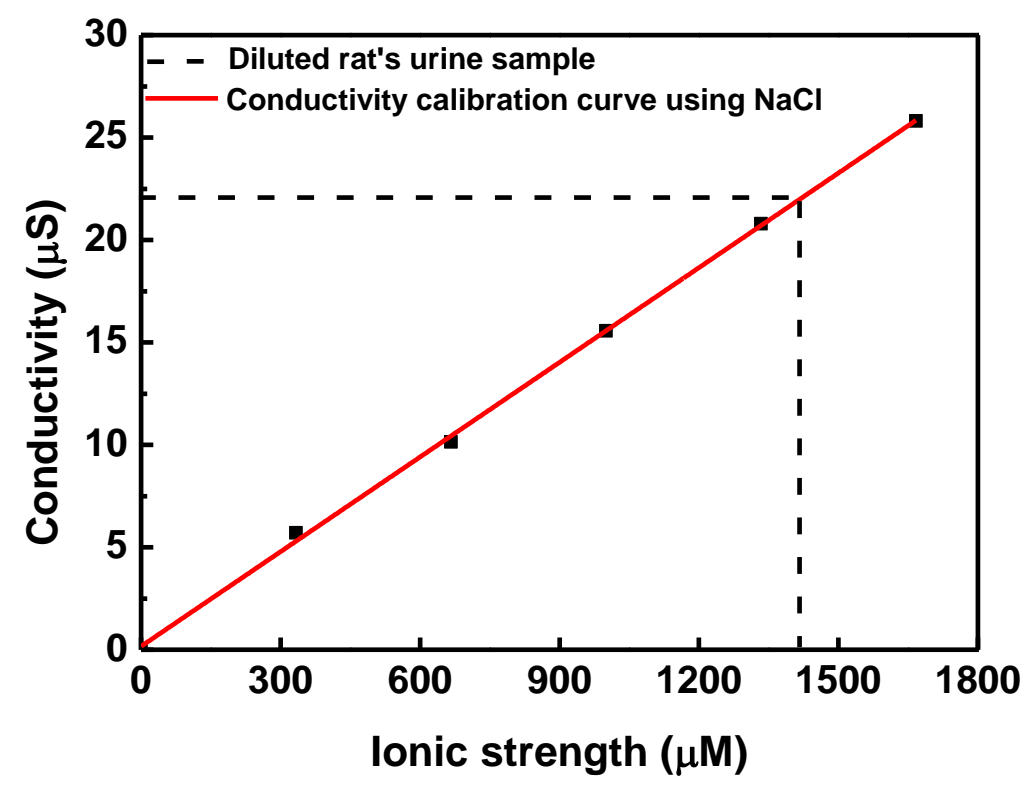

Figure B.5: a) Standard calibration curve of the conductivity of $\mathrm{NaCl}$ solutions to estimate the ionic strength of rat urine. $10 \mu \mathrm{L}$ of the rat urine was diluted to $15 \mathrm{~mL}$, using Milli-Q water, and the conductivity was measured. The final ionic strength of the rat urine sample was $2.1 \mathrm{mM}$.

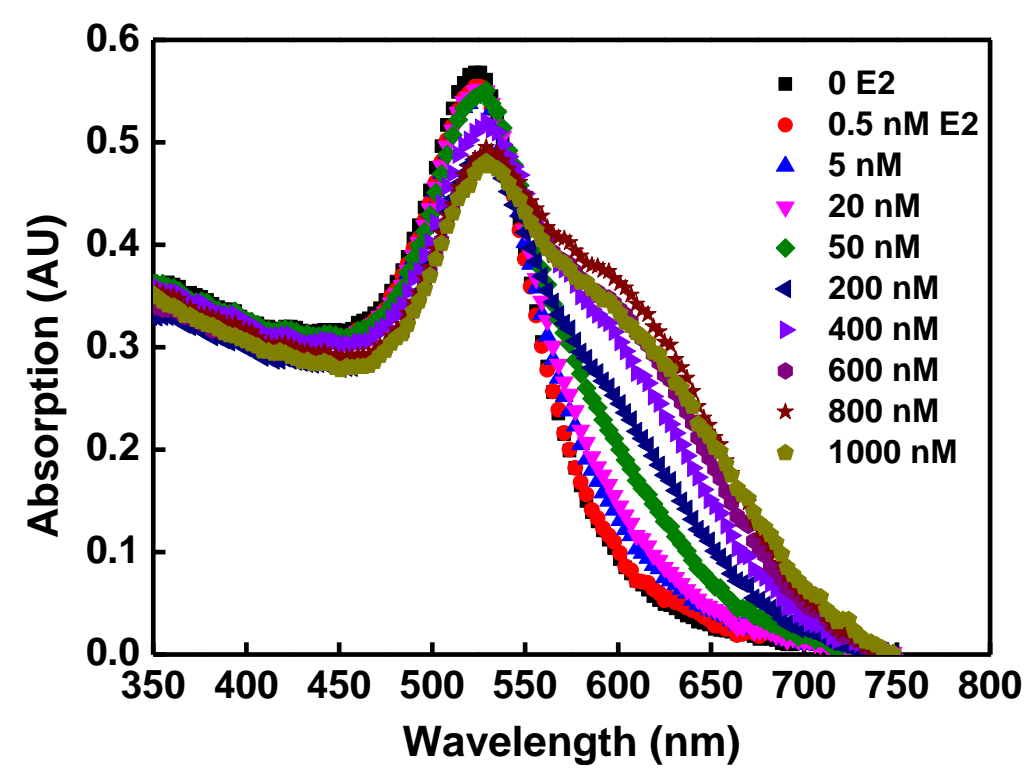

Figure B.6: Absorption spectra of E2 sensing in rat urine samples using AuNP-35mer aptamer 


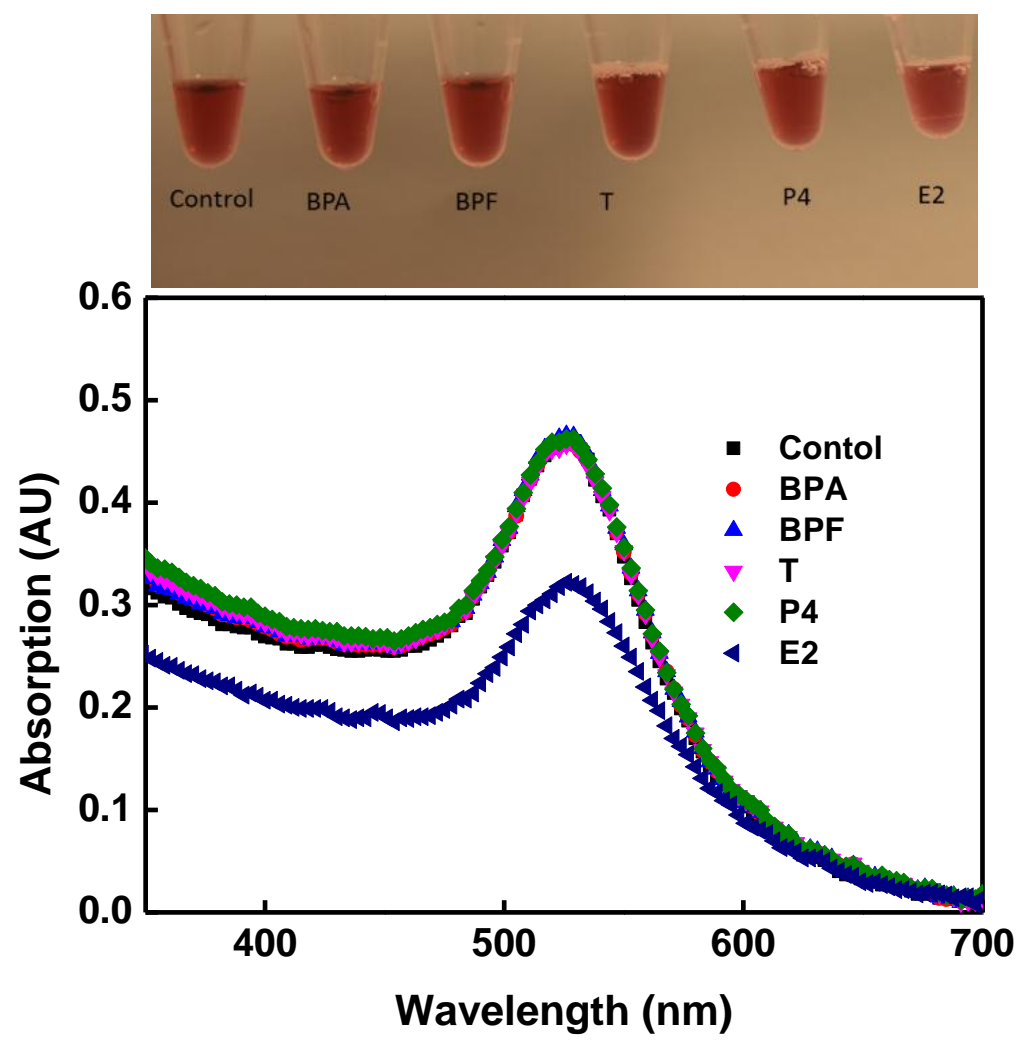

Figure B.7: Photograph (top) and UV-vis spectra of the specificity results using the 35-mer aptamer in rat urine samples spiked with $200 \mathrm{nM}$ BPA, BPF, progesterone (P4) and testosterone (T), and E2.

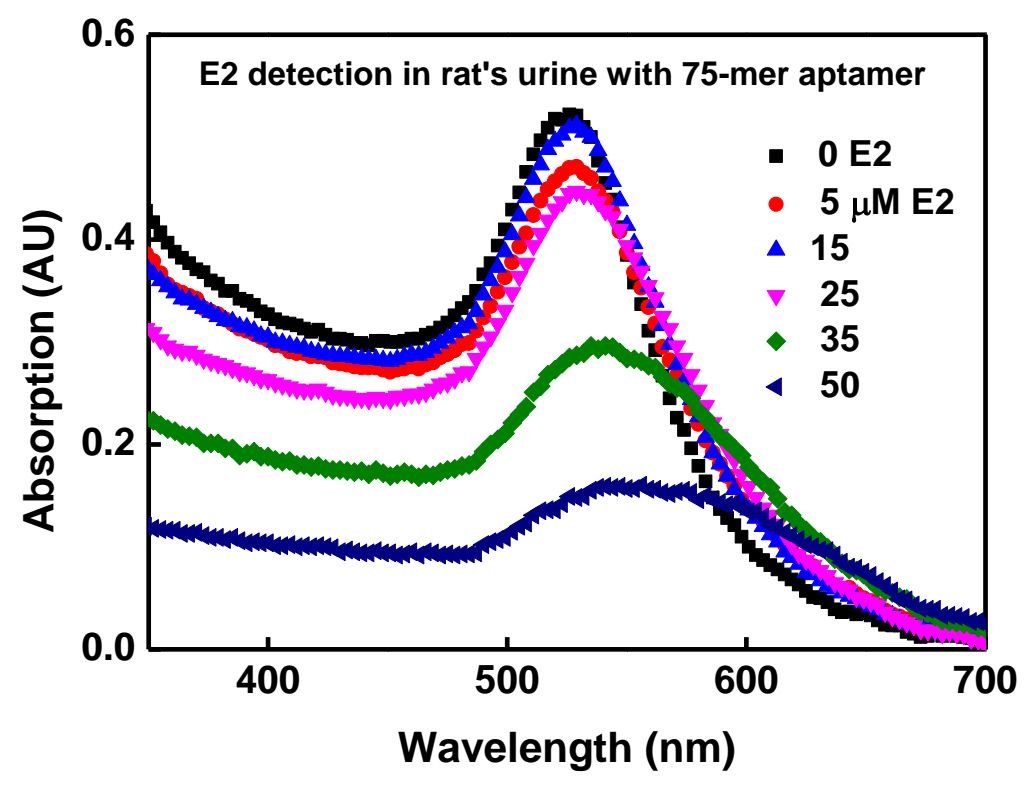

Figure B.8: Absorption spectra of E2 sensing in rat urine samples using the AuNP75-mer aptamer. 


\section{B.4 $K_{\mathrm{D}}$ measurements}
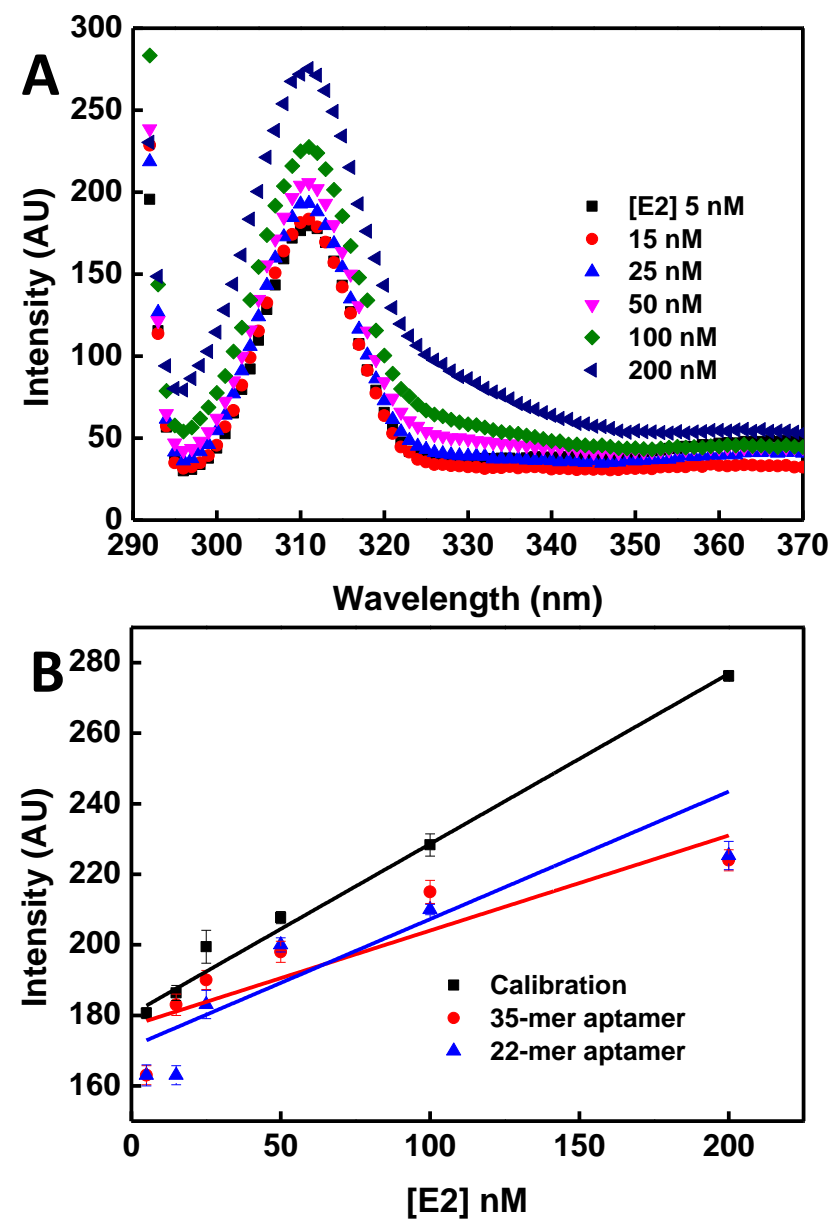

Figure B.9: a) Representative emission spectra of E2 in BWB. b) Calibration curve of E2 and fluorescent intensities of E2 after reaction with NP-22-mer and NP-35mer aptamers. 


\section{Appendix C}

\section{Supplementary information of EIS based sensor}

\section{C.1 Surface area of AuNPs on glassy carbon electrode}

The active AuNPs surface area deposited on glassy carbon electrode was estimated by the measurement of the peak intensity at a different scan rate (Fig. 4.6) and applying the Randles-Sevcik Eq. C.1 164:170|171

$$
i=2.68 \times 10^{5} n^{2 / 3} A D^{1 / 2} v^{1 / 2} c^{0}
$$

where $\mathrm{i}$ is peak current, A the electrode surface area, D the diffusion coefficient, $v$ the potential scan rate, and $\mathrm{c}^{0}$ the mediator concentration.

The bulk solution (mediator) employed for the experiments contained $1 \mathrm{mM}\left[\mathrm{Fe}(\mathrm{CN})_{6}\right]^{-3 /-4}$ in $0.1 \mathrm{M} \mathrm{KCl}$. The diffusion constant value $\left(\mathrm{D}=6.9 \times 10^{-6} \mathrm{~cm}^{2} \mathrm{~s}^{-1}\right.$ vs. literature quoted value $\left.7.210^{-6} \mathrm{~cm}^{2} \mathrm{~s}^{-1}\right)^{187}$ was ascertained from a known GCE surface area $\left(0.00785 \mathrm{~cm}^{2}\right)$ and calculation of $\mathrm{K}\left(5.6 \times 10^{-6}\right) \mathrm{A} \mathrm{s}^{1 / 2} / \mathrm{V}^{1 / 2}$ from (Fig. 4.6) and simplifying Eq. C.1 as follow

$$
i=k v^{1 / 2}
$$

where $\mathrm{k}=2.68 \times 10^{5} \mathrm{n}^{2 / 3} \mathrm{~A} \mathrm{D}^{1 / 2} \mathrm{c}^{0}$ 
Then the $\mathrm{k}$ value of the AuNPs modified electrode was measured to be $2.0 \times 10^{-5}$ $\mathrm{A} \mathrm{s}^{1 / 2} / \mathrm{V}^{1 / 2}$ (Fig. 4.6). From the measurement of $\mathrm{k}$, the surface area of AuNP electrode was found to be $0.0157 \mathrm{~cm}^{2}$. The roughness factor of the AuNP electrode was calculated to be 2 , assuming the underlying GCE is geometrically smooth.

\section{C.2 Figures for EIS AuNP electrode}
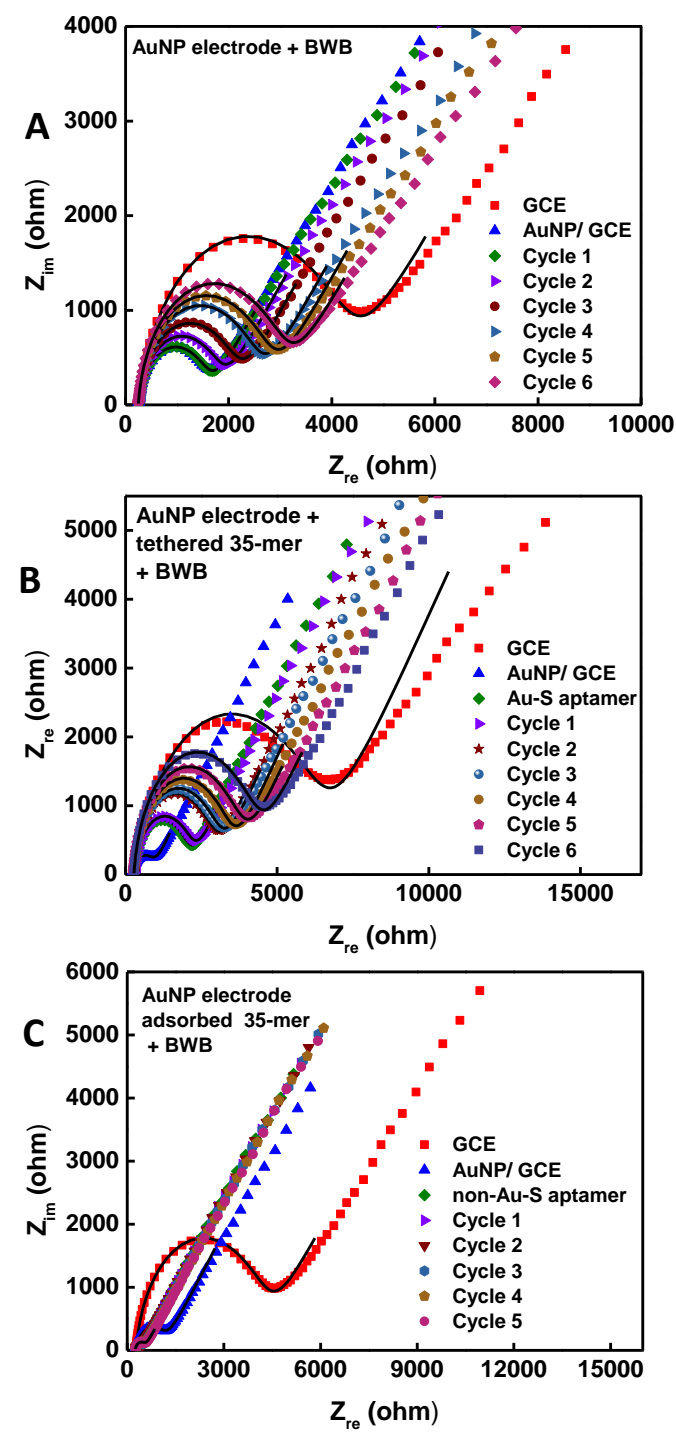

Figure C.1: EIS spectra presented as Nyquist plots characterizing the stability of different electrodes a) AuNP/ GCE, b) Covalently tethered 35-mer aptamer, and c) Non-specifically adsorbed 35-mer aptamer against incubation cycles with buffer (BWB). Experimental data (symbols) are fitted to the Randles cell equivalent circuit (lines). 

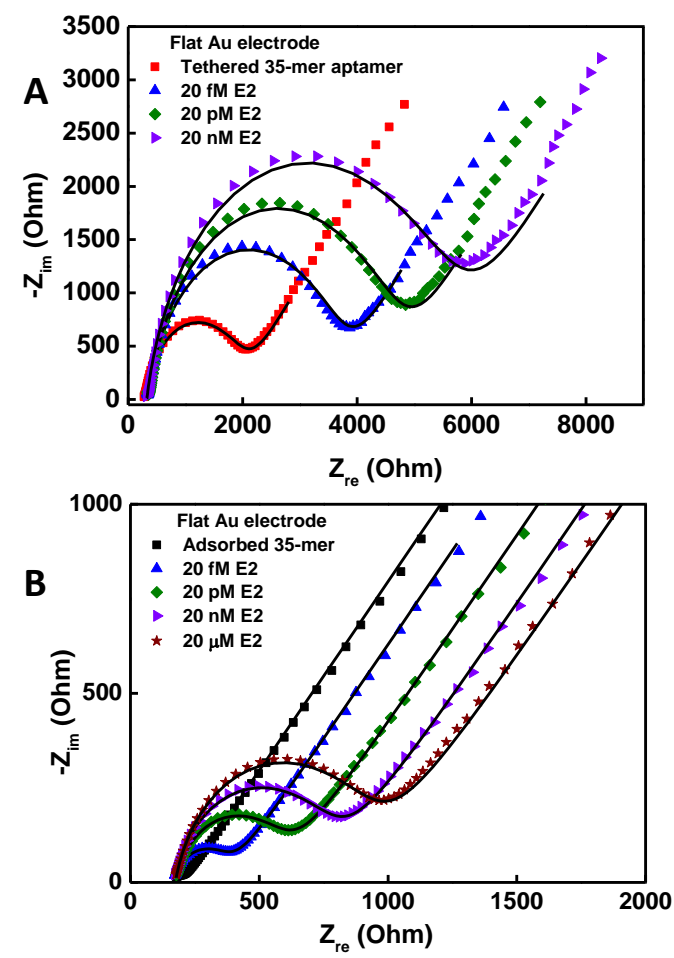

Figure C.2: EIS spectra presented as Nyquist plots characterizing the response towards E2 with the 35-mer aptamer covalently tethered to (a) and non-specifically adsorbed (b) on a flat Au electrode. Experimental data (symbols) are fitted to the Randles cell equivalent circuit (lines).

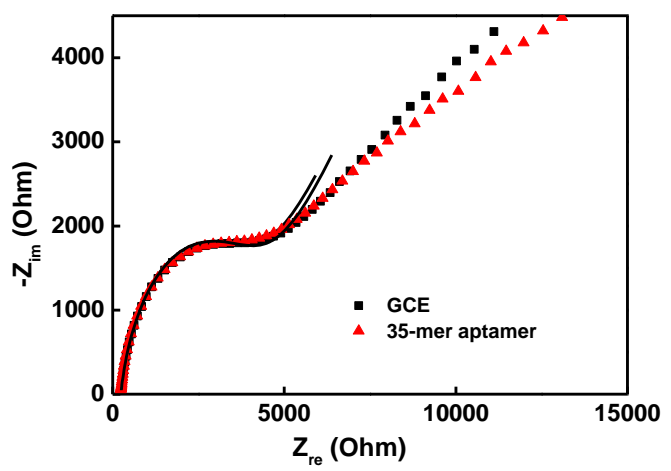

Figure C.3: Control experiment of exposing the 35-mer aptamer to GCE to validate the results with AuNP electrode. 


\section{Appendix D}

\section{Chemicals and materials}

\section{Size based aptasensor}

2-(N-morpholino)ethanesulfonic acid (MES), tris(hydroxymethyl) amino methane (Tris-HCl), N-(3-dimethylaminopropyl) N-ethylcarbodiimide hydrochloride (EDC), $\mathrm{N}$ hydroxy-succinimide (NHS), $\mathrm{NaCl}, \mathrm{MgCl}_{2}, \mathrm{CaCl}_{2}, \mathrm{KCl}$, polyoxyethylene (9) nonylphenylether (IGEPAL ${ }^{\circledR}$, non-ionic detergent), $17-\beta$ estradiol (E2), progesterone (P4), testosterone (T), 4, 4'-methylene diphenol (BPF), bisphenol-A (BPA) and adenosine were purchased from Sigma-Aldrich. The E2 aptamer was isolated by SELEX process from a random ssDNA library composed of approximately $7.2 \times 10^{14}$ DNA molecules. The random ssDNA library, biotin-aptamer and primers were purchased from Life Sciences, Australia.

The amino terminated E2 and adenosine (previously reported by (Kim et al., 123 ) aptamers were synthesized and PAGE purified by Sigma-Aldrich. For the fluorescence experiment to determine the aptamer density on NPs, a closely related ssDNA sequence of 76-mers previously used by Kim et al., ${ }^{7}$ for the detection of E2, was used after modification with 5'-amino $\left(\mathrm{CH}_{2}\right)_{6}$ and 3'-Cy5.5 groups (Faptamer) by Eurofins Genomics India Pvt. Ltd. All aptamers were rehydrated using deionized water (Milli-Q, 18.2 $\mathrm{M} \Omega \mathrm{cm}$ ) and kept at $-5^{\circ} \mathrm{C}$ before use (unless stated). Standard $217 \mathrm{~nm}$ carboxylated polystyrene NPs (Bangs Laboratories, Indiana, USA) were purchased unmodified from Izon Science (Christchurch, New Zealand). The NPs are nominally supplied with a mode size of $217 \mathrm{~nm}$ and a solid content of $10.1 \mathrm{wt} \%$, corresponding to a concentration of $1.763 \times 10^{14}$ particles $\mathrm{g}^{-1}\left(1.79 \times 10^{17}\right.$ particles $\left.\mathrm{mL}^{-1}\right)$. The carboxyl group concentration in surface titration is $86 \mu \mathrm{eq} g^{-1}$ (corresponding to $5 \mathrm{nmol}$ of carboxylates for every 1.04 
$x 10^{10}$ particles). All other chemicals used were of analytical grade. Deionized water (Milli-Q, $18.2 \mathrm{M} \Omega \mathrm{cm}$ ) was used for all solution preparation.

\section{Colorimetric aptasensor}

Chloroauricacid $\left(\mathrm{HAuCl}_{4}\right)$ was purchased from Sigma-Aldrich. E2 75-mer aptamer, truncated versions 35-mer and 22-mer aptamers, random 75-mer DNA, random 35-mer DNA, random 22-mer DNA, and the polyT20-35-mer-polyT20 sequence were purchased from Alpha DNA. Sequences are given in Table D.1 in Appendix D. The 35-mer and 22-mer were synthesised with $5^{\prime}-\mathrm{NH}_{2}\left(\mathrm{CH}_{2}\right)_{6}$ for covalent attachment with carboxylated polystyrene NPs (for $\mathrm{K}_{\mathrm{D}}$ measurements). The ssDNA were dissolved in deionized water (Milli-Q, $18.2 \mathrm{M} \Omega \mathrm{cm}$ ) and kept at $-5{ }^{\circ} \mathrm{C}$ before use. Deionized water (Milli-Q, 18.2 M $\mathrm{cm}$ ) water was used in all experiments (unless stated) and all other chemicals were of analytical grade.

\section{Electrochemical impedance based aptasensor}

Phosphate buffered saline (PBS, pH 7.4, $137 \mathrm{mM} \mathrm{NaCl}, 2.7 \mathrm{mM} \mathrm{KCl}, 10 \mathrm{mM}$ $\mathrm{Na}_{2} \mathrm{HPO}_{4}, 1.8 \mathrm{mM} \mathrm{KH}_{2} \mathrm{PO}_{4}$ ), 6-mercaptohexanoic acid (MHA), and bovine serum albumin (BSA) were purchased from Sigma-Aldrich. The thiolated and nonthiolated DNA sequences used in this study were synthesised and PAGE purified by Alpha DNA. The thiolated DNAs were treated with dithiothreitol (DTT) at the last step of the synthesis, by Alpha DNA, to reduce the S-S bond and DTT was removed by ethanol precipitation after which the DNAs were lyophilized and sealed under Argon. All DNAs were rehydrated using deionized water (Milli-Q, 18.2 $\mathrm{M} \Omega \mathrm{cm}$ ) and kept at $-5{ }^{\circ} \mathrm{C}$ for subsequent dilutions. Sequences used in this study are given in Table D.1 in Appendix D. Note that the 75-mer and 35-mer aptamers were modified at the $5^{\prime}$ end with $\mathrm{SH}\left(\mathrm{CH}_{2}\right)_{6}$ for covalent attachment with the Au surface. All aqueous solutions were prepared using deionized water (Milli-Q, 18.2 M $\Omega \mathrm{cm}$ ). Other chemicals in this study were of analytical grade and used as supplied unless otherwise stated. 
Table D.1: Sequences used in this thesis (left to right $5^{\prime}$ end and $3^{\prime}$ end). The sequences were modified with the $5^{\prime}-\mathrm{NH}_{2}\left(\mathrm{CH}_{2}\right)_{6}$ or $\mathrm{SH}\left(\mathrm{CH}_{2}\right)_{6}$ when covalent coupling was needed.

75-mer aptamer

35-mer aptamer

22-mer aptamer

75-mer random DNA

35-mer random DNA
ATACGAGCTTGTTCAATACGAAGGGATGCCGTTTGGGC CCAAGTTCGGCATAGTGTGGTGATAGTAAGAGCAATC

AAGGGATGCCGTTTGGGCCCAAGTTCGGCATAGTG

GCCGTTTGGGCCCAAGTTCGGC

AGGCCTAAGGGCATAATTAGCTCGAGCTCGAAAGGG

GTTATATGATGATTTGAATTCATGGGGCCCGACTCGGAT

ACGGGTGGCCGCCAGGTCTTGAAGTGGCAGTATTA

TGGGCCCTTTACGGACCGCGTG 


\section{Bibliography}

1. Mitchell, J. S.; Wu, Y. Surface plasmon resonance biosensors for highly sensitive detection of small biomolecules; INTECH Open Access Publisher, 2010.

2. Bock, L. C.; Griffin, L. C.; Latham, J. A.; Vermaas, E. H.; Toole, J. J. Nature 1992, 355, 564-566.

3. Sefah, K.; Meng, L.; Lopez-Colon, D.; Jimenez, E.; Liu, C. et al. PLoS One 2010, 5, e14269.

4. Lee, J. H.; Yigit, M. V.; Mazumdar, D.; Lu, Y. Advanced drug delivery reviews 2010, 62, 592-605.

5. Wang, L.; Liu, X.; Hu, X.; Song, S.; Fan, C. Chemical Communications 2006, 3780-3782.

6. Tombelli, S.; Minunni, M.; Mascini, M. Biomolecular Engineering 2007, 24, 191-200.

7. Kim, Y. S.; Jung, H. S.; Matsuura, T.; Lee, H. Y.; Kawai, T. et al. Biosensors and Bioelectronics 2007, 22, 2525-2531.

8. Kato, T.; Takemura, T.; Yano, K.; Ikebukuro, K.; Karube, I. Biochimica et Biophysica Acta 2000, 1493, 12-18.

9. McKeague, M.; DeRosa, M. C. Journal of nucleic acids 2012, 2012.

10. Song, S.; Wang, L.; Li, J.; Fan, C.; Zhao, J. Trends in Analytical Chemistry 2008, $27,108-117$.

11. Mairal, T.; Özalp, V. C.; Sánchez, P. L.; Mir, M.; Katakis, I. et al. Analytical and bioanalytical chemistry 2008, 390, 989-1007.

12. Cheng, A. K.; Sen, D.; Yu, H.-Z. Bioelectrochemistry 2009, 77, 1-12.

13. Zhang, X.; Guo, Q.; Cui, D. Sensors 2009, 9, 1033-1053.

14. Lee, S. H.; Sung, J. H.; Park, T. H. Annals of biomedical engineering 2012, 40, 1384-1397. 
15. Jianrong, C.; Yuqing, M.; Nongyue, H.; Xiaohua, W.; Sijiao, L. Biotechnology advances 2004, 22, 505-518.

16. Liu, J. Physical Chemistry Chemical Physics 2012, 14, 10485-10496.

17. Kornberg, A.; Baker, T. A. DNA replication; WH Freeman San Francisco, 1980.

18. Tuerk, C.; Gold, L. Science 1990, 249, 505-510.

19. Ellington, A. D.; Szostak, J. W. nature 1990, 346, 818-822.

20. Gopinath, S. C. Aptamers; Wiley Online Library, 2011.

21. Stoltenburg, R.; Reinemann, C.; Strehlitz, B. Biomolecular engineering 2007, 24, 381-403.

22. Hermann, T.; Patel, D. J. Science 2000, 287, 820-825.

23. Alsager, O. A.; Kumar, S.; Willmott, G. R.; McNatty, K. P.; Hodgkiss, J. M. Biosensors and Bioelectronics 2014, 57, 262-268.

24. Mann, D.; Reinemann, C.; Stoltenburg, R.; Strehlitz, B. Biochemical and biophysical research communications 2005, 338, 1928-1934.

25. Maehashi, K.; Katsura, T.; Kerman, K.; Takamura, Y.; Matsumoto, K. et al. Analytical Chemistry 2007, 79, 782-787.

26. Jayasena, S. D. Clinical chemistry 1999, 45, 1628-1650.

27. Posthuma-Trumpie, G. A.; Korf, J.; van Amerongen, A. Analytical and bioanalytical chemistry 2009, 393, 569-582.

28. Tan, W.; Wang, H.; Chen, Y.; Zhang, X.; Zhu, H. et al. Trends in biotechnology 2011, 29, 634-640.

29. Levy, M.; Cater, S. F.; Ellington, A. D. ChemBioChem 2005, 6, 2163-2166.

30. Chen, J.; Fang, Z.; Liu, J.; Zeng, L. Food Control 2012, 25, 555-560.

31. Stojanovic, M. N.; De Prada, P.; Landry, D. W. Journal of the American Chemical Society 2001, 123, 4928-4931.

32. Yamamoto, R.; Kumar, P. K. Genes to cells 2000, 5, 389-396.

33. Wang, S.-e.; Si, S. Analytical Methods 2013, 5, 2947-2953.

34. Eustis, S.; El-Sayed, M. A. Chemical society reviews 2006, 35, 209-217.

35. Li, Y.; Schluesener, H. J.; Xu, S. Gold Bulletin 2010, 43, 29-41. 
36. Zhao, W.; Brook, M. A.; Li, Y. ChemBioChem 2008, 9, 2363-2371.

37. Ghosh, S. K.; Pal, T. Chemical Reviews 2007, 107, 4797-4862.

38. Gopinath, S. C.; Lakshmipriya, T.; Awazu, K. Biosensors and Bioelectronics 2014, 51, 115-123.

39. Kimling, J.; Maier, M.; Okenve, B.; Kotaidis, V.; Ballot, H. et al. The Journal of Physical Chemistry B 2006, 110, 15700-15707.

40. Turkevich, J.; Stevenson, P. C.; Hillier, J. Discussions of the Faraday Society 1951, 11, 55-75.

41. Li, H.; Rothberg, L. J. Journal of the American Chemical Society 2004, 126, 10958-10961.

42. Li, H.; Rothberg, L. Proceedings of the National Academy of Sciences of the United States of America 2004, 101, 14036-14039.

43. Mei, Z.; Chu, H.; Chen, W.; Xue, F.; Liu, J. et al. Biosensors and Bioelectronics 2013, 39, 26-30.

44. Yang, C.; Wang, Y.; Marty, J.-L.; Yang, X. Biosensors and Bioelectronics 2011, 26, 2724-2727.

45. Wei, H.; Li, B.; Li, J.; Wang, E.; Dong, S. Chemical Communications 2007, 37353737.

46. Peng, Y.; Li, L.; Mu, X.; Guo, L. Sensors and Actuators B: Chemical 2013, 177, 818-825.

47. Liu, J.; Lu, Y. Angewandte Chemie 2006, 118, 96-100.

48. Huizenga, D. E.; Szostak, J. W. Biochemistry 1995, 34, 656-665.

49. Cekan, P.; Jonsson, E. Ö.; Sigurdsson, S. T. Nucleic acids research 2009, 37, 3990-3995.

50. Ge, X.; Asiri, A. M.; Du, D.; Wen, W.; Wang, S. et al. Trends in Analytical Chemistry 2014, 58, 31-39.

51. Sajid, M.; Kawde, A.-N.; Daud, M. Journal of Saudi Chemical Society 2014, [In press].

52. Dzantiev, B. B.; Byzova, N. A.; Urusov, A. E.; Zherdev, A. V. Trends in Analytical Chemistry 2014, 55, 81-93. 
53. Ngom, B.; Guo, Y.; Wang, X.; Bi, D. Analytical and bioanalytical chemistry 2010, 397, 1113-1135.

54. Goryacheva, I. Y.; Lenain, P.; De Saeger, S. Trends in Analytical Chemistry 2013, $46,30-43$.

55. Wang, L.; Ma, W.; Chen, W.; Liu, L.; Ma, W. et al. Biosensors and Bioelectronics 2011, 26, 3059-3062.

56. Suni, I. I. Trends in Analytical Chemistry 2008, 27, 604-611.

57. Barsoukov, E.; Macdonald, J. R. Impedance spectroscopy: theory, experiment, and applications; John Wiley \& Sons, 2005.

58. Hianik, T.; Wang, J. Electroanalysis 2009, 21, 1223-1235.

59. Velasco-Garcia, M.; Missailidis, S. Gene Therapy and Molecular Biology 2009, $13,1-10$.

60. de-los Santos-Álvarez, N.; Lobo-Castañón, M.; Miranda-Ordieres, A. J.; Tuñón-Blanco, P. Trends in Analytical Chemistry 2008, 27, 437-446.

61. Xu, Y.; Cheng, G.; He, P.; Fang, Y. Electroanalysis 2009, 21, 1251-1259.

62. Radi, A.-E. International Journal of Electrochemistry 2011, 2011.

63. Wu, J.; Chu, H.; Mei, Z.; Deng, Y.; Xue, F. et al. Analytica chimica acta 2012, 753, 27-31.

64. Xiao, Y.; Lubin, A. A.; Heeger, A. J.; Plaxco, K. W. Angewandte Chemie International Edition 2005, 44, 5456-5459.

65. Radi, A.-E.; Acero S'nchez, J. L.; Baldrich, E.; O'Sullivan, C. K. Journal of the American Chemical Society 2006, 128, 117-124.

66. O'Sullivan, C. K.; Radi, A.-E. Chemical communications 2006, 3432-3434.

67. Castillo, G.; Lamberti, I.; Mosiello, L.; Hianik, T. Electroanalysis 2012, 24, 512520.

68. Lin, Z.; Chen, L.; Zhang, G.; Liu, Q.; Qiu, B. et al. Analyst 2012, 137, 819-822.

69. Pilehvar, S.; Dierckx, T.; Blust, R.; Breugelmans, T.; De Wael, K. Sensors 2014, 14, 12059-12069.

70. Shen, L.; Chen, Z.; Li, Y.; Jing, P.; Xie, S. et al. Chemical Communications 2007, 2169-2171. 
71. Xue, F.; Wu, J.; Chu, H.; Mei, Z.; Ye, Y. et al. Microchimica Acta 2013, 180, 109-115.

72. Li, B.; Du, Y.; Wei, H.; Dong, S. Chemical Communications 2007, 3780-3782.

73. Yang, X.; Huang, J.; Wang, Q.; Wang, K.; Yang, L. et al. Analytical Methods 2011, 3, 59-61.

74. Wanekaya, A.; DeLong, R.; Datta, B. Aptamer-Based Device For Detection Of Cancer Markers And Methods Of Use. 2011; US Patent App. 13/250,451.

75. Platt, M.; Willmott, G. R.; Lee, G. U. Small 2012, 8, 2436-2444.

76. Rinker, S.; Ke, Y.; Liu, Y.; Chhabra, R.; Yan, H. Nature nanotechnology 2008, 3, 418-422.

77. Cruz-Aguado, J. A.; Penner, G. Journal of agricultural and food chemistry 2008, $56,10456-10461$.

78. Lau, P. S.; Coombes, B. K.; Li, Y. Angewandte Chemie International Edition 2010, $49,7938-7942$.

79. Pape-Zambito, D.; Roberts, R.; Kensinger, R. Journal of dairy science 2010, 93, 2533-2540.

80. Taioli, E.; Im, A.; Xu, X.; Veenstra, T. D.; Ahrendt, G. et al. Reproductive Biology and Endocrinology 2010, 8, 93.

81. Sarmah, A.; Northcott, G.; Leusch, F.; Tremblay, L. Science of the Total Environment 2006, 355, 135-144.

82. Petrovic, M.; Eljarrat, E.; De Alda, M. L.; Barceló, D. Analytical and Bioanalytical Chemistry 2004, 378, 549-562.

83. Leet, J. K.; Gall, H. E.; Sepúlveda, M. S. Journal of Applied Toxicology 2011, 31, 379-398.

84. Menditto, A.; Turrio-Baldassarri, L. Chemosphere 1999, 39, 1301-1307.

85. Auriol, M.; Filali-Meknassi, Y.; Tyagi, R. D.; Adams, C. D.; Surampalli, R. Y. Process Biochemistry 2006, 41, 525-539.

86. Li, Y.; Luh, C. J.; Burns, K. A.; Arao, Y.; Jiang, Z. et al. Environmental health perspectives 2013, 121, 459-466.

87. Briciu, R. D.; Kot-Wasik, A.; Namiesnik, J. Journal of chromatographic science 2009, 47, 127-139. 
88. Kuster, M.; López de Alda, M. J.; Hernando, M. D.; Petrovic, M.; MartínAlonso, J. et al. Journal of Hydrology 2008, 358, 112-123.

89. Snyder, S. A.; Keith, T. L.; Verbrugge, D. A.; Snyder, E. M.; Gross, T. S. et al. Environmental Science \& Technology 1999, 33, 2814-2820.

90. Jeannot, R.; Sabik, H.; Sauvard, E.; Dagnac, T.; Dohrendorf, K. Journal of Chromatography A 2002, 974, 143-159.

91. Keefe, A. D.; Pai, S.; Ellington, A. Nature Reviews Drug Discovery 2010, 9, 537-550.

92. Lee, J.-O.; So, H.-M.; Jeon, E.-K.; Chang, H.; Won, K. et al. Analytical and bioanalytical chemistry 2008, 390, 1023-1032.

93. Zhou, J.; Battig, M. R.; Wang, Y. Analytical and bioanalytical chemistry 2010, $398,2471-2480$.

94. Tong, P.; Zhang, L.; Xu, J.-J.; Chen, H.-Y. Biosensors and Bioelectronics 2011, 29, 97-101.

95. Kozak, D.; Anderson, W.; Grevett, M.; Trau, M. The Journal of Physical Chemistry C 2012, 116, 8554-8561.

96. Willmott, G.; Moore, P. Nanotechnology 2008, 19, 475504.

97. Willmott, G. R.; Parry, B. E. Journal of Applied Physics 2011, 109, 094307.

98. Weatherall, E.; Willmott, G. R. Analyst 2015, [In press].

99. Kozak, D.; Anderson, W.; Vogel, R.; Trau, M. Nano today 2011, 6, 531-545.

100. Vogel, R.; Willmott, G.; Kozak, D.; Roberts, G. S.; Anderson, W. et al. Analytical chemistry 2011, 83, 3499-3506.

101. Blundell, E. L.; Mayne, L. J.; Billinge, E. R.; Platt, M. Analytical Methods 2015, [In press].

102. Pecora, R. Dynamic light scattering: applications of photon correlation spectroscopy; Springer, 1985.

103. Lorber, B.; Fischer, F.; Bailly, M.; Roy, H.; Kern, D. Biochemistry and Molecular Biology Education 2012, 40, 372-382.

104. Kirby, B. J.; Hasselbrink, E. F. Electrophoresis 2004, 25, 187-202.

105. Glawdel, T.; Ren, C. Encyclopedia of Microfluidics and Nanofluidics; Springer, 2008; pp 2199-2207. 
106. Tantra, R.; Schulze, P.; Quincey, P. Particuology 2010, 8, 279-285.

107. Anderson, W.; Kozak, D.; Coleman, V. A.; Jämting, s. K.; Trau, M. Journal of colloid and interface science 2013, 405, 322-330.

108. Jenison, R. D.; Gill, S. C.; Pardi, A.; Polisky, B. Science 1994, 263, 1425-1429.

109. Zuker, M. Nucleic acids research 2003, 31, 3406-3415.

110. Jing, M.; Bowser, M. T. Analytica chimica acta 2011, 686, 9-18.

111. Jo, M.; Ahn, J.-Y.; Lee, J.; Lee, S.; Hong, S. W. et al. Oligonucleotides 2011, 21, 85-91.

112. Greenfield, N. J. Methods in enzymology 2004, 383, 282.

113. Siligardi, G.; Hussain, R.; Berova, N.; Vorlíčková, M.; Kejnovská, I. et al. Chirality 2012, 24, 691-698.

114. Chang, Y.-M.; Chen, C. K.-M.; Chang, Y.-C.; Jeng, W.-Y.; Hou, M.-H. et al. PloS one 2012, 7, e45665.

115. Zheng, Y.; Wang, Y.; Yang, X. Sensors and Actuators B: Chemical 2011, 156, 95-99.

116. Somerville, J. A.; Willmott, G. R.; Eldridge, J.; Griffiths, M.; McGrath, K. M. Journal of colloid and interface science 2013, 394, 243-251.

117. Finsy, R. Advances in colloid and interface science 1994, 52, 79-143.

118. Xu, P. F.; Hung, A. M.; Noh, H.; Cha, J. N. Small 2013, 9, 228-232.

119. Roberts, G. S.; Kozak, D.; Anderson, W.; Broom, M. F.; Vogel, R. et al. Small 2010, 6, 2653-2658.

120. Russo, J.; Fernandez, S. V.; Russo, P. A.; Fernbaugh, R.; Sheriff, F. S. et al. The FASEB journal 2006, 20, 1622-1634.

121. Olowu, R. A.; Ndangili, P. M.; Baleg, A. A.; Ikpo, C. O.; Njomo, N. et al. International Journal of Electrochemical Science 2011, 6, 1686-1708.

122. Yildirim, N.; Long, F.; Gao, C.; He, M.; Shi, H.-C. et al. Environmental science E technology 2012, 46, 3288-3294.

123. Jinheung, K.; In, Y. K.; Min, S. C.; Qiong, W. Chemical Communications 2009, 4747-4749.

124. Kelly, S. M.; Jess, T. J.; Price, N. C. Biochimica et Biophysica Acta 2005, 1751, 119-139. 
125. Yang, Y.; Lai, E. P.; Liu, M. Open Colloid Science Journal 2010, 3, 1-8.

126. Kim, Y. S.; Kim, J. H.; Kim, I. A.; Lee, S. J.; Jurng, J. et al. Biosensors and Bioelectronics 2010, 26, 1644-1649.

127. Song, K.-M.; Cho, M.; Jo, H.; Min, K.; Jeon, S. H. et al. Analytical biochemistry 2011, 415, 175-181.

128. He, J.; Liu, Y.; Fan, M.; Liu, X. Journal of agricultural and food chemistry 2011, $59,1582-1586$.

129. Shi, H.; Zhao, G.; Liu, M.; Fan, L.; Cao, T. Journal of hazardous materials 2013, 260, 754-761.

130. Chávez, J. L.; MacCuspie, R. I.; Stone, M. O.; Kelley-Loughnane, N. Journal of Nanoparticle Research 2012, 14, 1-11.

131. Randazzo, A.; Spada, G. P.; da Silva, M. W. Quadruplex Nucleic Acids; Springer, 2013; pp 67-86.

132. McManus, S. A.; Li, Y. PloS one 2013, 8, e64131.

133. Steel, A. B.; Herne, T. M.; Tarlov, M. J. Analytical Chemistry 1998, 70, 46704677.

134. Elavarasi, M.; Rajeshwari, A.; Chandrasekaran, N.; Mukherjee, A. Analytical Methods 2013, 5, 6211-6218.

135. Jiang, L.; Guan, J.; Zhao, L.; Li, J.; Yang, W. Colloids and Surfaces A: Physicochemical and Engineering Aspects 2009, 346, 216-220.

136. deCatanzaro, D.; Beaton, E. A.; Khan, A.; Vella, E. Reproduction 2006, 132, 309-317.

137. Grabar, K. C.; Freeman, R. G.; Hommer, M. B.; Natan, M. J. Analytical chemistry 1995, 67, 735-743.

138. Haiss, W.; Thanh, N. T.; Aveyard, J.; Fernig, D. G. Analytical chemistry 2007, $79,4215-4221$.

139. Lisdat, F.; Schäfer, D. Analytical and bioanalytical chemistry 2008, 391, 15551567.

140. Degefa, T. H.; Kwak, J. Journal of Electroanalytical Chemistry 2008, 612, 37-41.

141. Park, J.-Y.; Park, S.-M. Sensors 2009, 9, 9513-9532. 
142. Kannan, B.; Williams, D. E.; Booth, M. A.; Travas-Sejdic, J. Analytical chemistry 2011, 83, 3415-3421.

143. Booth, M. A.; Harbison, S.; Travas-Sejdic, J. Biosensors and Bioelectronics 2011, $28,362-367$.

144. Travas-Sejdic, J.; Aydemir, N.; Kannan, B.; Williams, D.; Malmström, J. Journal of Materials Chemistry B 2014, 2, 4593-4609.

145. Shamsi, M. H.; Kraatz, H.-B. Analyst 2010, 135, 2280-2285.

146. Gebala, M.; Schuhmann, W. Physical Chemistry Chemical Physics 2012, 14, 14933-14942.

147. Peng, H.; Soeller, C.; Travas-Sejdic, J. Macromolecules 2007, 40, 909-914.

148. Peng, H.; Soeller, C.; Vigar, N. A.; Caprio, V.; Travas-Sejdic, J. Biosensors and Bioelectronics 2007, 22, 1868-1873.

149. Zhu, B.; Booth, M. A.; Shepherd, P.; Sheppard, A.; Travas-Sejdic, J. Biosensors and Bioelectronics 2015, 64, 74-80.

150. Brown, K. A.; Park, S.; Hamad-Schifferli, K. The Journal of Physical Chemistry C 2008, 112, 7517-7521.

151. Beaucage, S. L. Current medicinal chemistry 2001, 8, 1213-1244.

152. Xiao, Y.; Plaxco, K. W. Functional Nucleic Acids for Analytical Applications; Springer, 2009; pp 179-197.

153. Zayats, M.; Huang, Y.; Gill, R.; Ma, C.-a.; Willner, I. Journal of the American Chemical Society 2006, 128, 13666-13667.

154. Mahmoud, A. M.; Tang, T.; Harrison, D. J.; Lee, W. E.; Jemere, A. B. Biosensors and Bioelectronics 2014, 56, 328-333.

155. Herne, T. M.; Tarlov, M. J. Journal of the American Chemical Society 1997, 119, 8916-8920.

156. Petrovykh, D. Y.; Kimura-Suda, H.; Whitman, L. J.; Tarlov, M. J. Journal of the American Chemical Society 2003, 125, 5219-5226.

157. Wong, I. Y.; Melosh, N. A. Biophysical journal 2010, 98, 2954-2963.

158. Lao, R.; Song, S.; Wu, H.; Wang, L.; Zhang, Z. et al. Analytical chemistry 2005, $77,6475-6480$.

159. Sandström, P.; Boncheva, M.; Åkerman, B. Langmuir 2003, 19, 7537-7543. 
160. Xia, F.; Zuo, X.; Yang, R.; Xiao, Y.; Kang, D. et al. Proceedings of the National Academy of Sciences 2010, 107, 10837-10841.

161. Fan, L.; Zhao, G.; Shi, H.; Liu, M.; Li, Z. Biosensors and Bioelectronics 2013, 43, 12-18.

162. Liu, X.; Qin, Y.; Deng, C.; Xiang, J.; Li, Y. Talanta 2015, 132, 150-154.

163. Tymoczko, J.; Schuhmann, W.; Gebala, M. ACS applied materials $\mathcal{E}$ interfaces 2014, 6, 21851-21858.

164. Bard, A. J.; Faulkner, L. R. Electrochemical methods: fundamentals and applications; Wiley New York, 1980; Vol. 2.

165. Kilic, M. S.; Bazant, M. Z.; Ajdari, A. Physical review E 2007, 75, 021502.

166. Davies, J. T. Interfacial Phenomena (second edition); Elsevier, 2012.

167. Li, X.; Shen, L.; Zhang, D.; Qi, H.; Gao, Q. et al. Biosensors and Bioelectronics 2008, 23, 1624-1630.

168. Lin, X.; Ni, Y.; Kokot, S. Analytica chimica acta 2013, 765, 54-62.

169. Singh, K.; Rahman, M. A.; Son, J. I.; Kim, K. C.; Shim, Y.-B. Biosensors and Bioelectronics 2008, 23, 1595-1601.

170. Hrapovic, S.; Liu, Y.; Male, K. B.; Luong, J. H. Analytical chemistry 2004, 76, 1083-1088.

171. Jarzabek, G.; Borkowska, Z. Electrochimica acta 1997, 42, 2915-2918.

172. El-Cheick, F.; Rashwan, F.; Mahmoud, H.; El-Rouby, M. Journal of Solid State Electrochemistry 2010, 14, 1425-1443.

173. Kimura-Suda, H.; Petrovykh, D. Y.; Tarlov, M. J.; Whitman, L. J. Journal of the American Chemical Society 2003, 125, 9014-9015.

174. Zhang, L.; Jiang, X.; Wang, E.; Dong, S. Biosensors and Bioelectronics 2005, 21, 337-345.

175. Zhang, J.-J.; Cao, J.-T.; Shi, G.-F.; Huang, K.-J.; Liu, Y.-M. et al. Analytical Methods 2014, 6, 6796-6801.

176. Long, F.; Shi, H.; Wang, H. RSC Advances 2014, 4, 2935-2941.

177. Zhang, X.; Servos, M. R.; Liu, J. Journal of the American Chemical Society 2012, 134, 7266-7269. 
178. Ke, H.; Liu, M.; Zhuang, L.; Li, Z.; Fan, L. et al. Electrochimica Acta 2014, 137, 146-153.

179. Fan, L.; Zhao, G.; Shi, H.; Liu, M.; Wang, Y. et al. Environmental science E technology 2014, 48, 5754-5761.

180. Valentini, F.; Compagnone, D.; Gentili, A.; Palleschi, G. Analyst 2002, 127, 1333-1337.

181. Alsager, O. A.; Kumar, S.; Zhu, B.; Travas-Sejdic, J.; McNatty, K. P. et al. Analytical chemistry 2015, 87, 4201-4209.

182. Zhu, B.; Alsager, O. A.; Kumar, S.; Hodgkiss, J. M.; Travas-Sejdic, J. Biosensors and Bioelectronics 2015, 70, 398-403.

183. Grozio, A.; Gonzalez, V. M.; Millo, E.; Sturla, L.; Vigliarolo, T. et al. Nucleic acid therapeutics 2013, 23, 322-331.

184. Martin, J. A.; Chávez, J. L.; Chushak, Y.; Chapleau, R. R.; Hagen, J. et al. Analytical and bioanalytical chemistry 2014, 406, 4637-4647.

185. Contreras Jiménez, G.; Eissa, S. H. H.; Ng, A.; Alhadrami, H.; Zourob, M. M. et al. Analytical chemistry 2015, 87, 1075-1082.

186. Dominguez-Medina, S.; Blankenburg, J.; Olson, J.; Landes, C. F.; Link, S. ACS sustainable chemistry $\mathcal{E}$ engineering 2013, 1, 833-842.

187. Konopka, S.; McDuffie, B. Analytical Chemistry 1970, 42, 1741-1746. 